\title{
SYNTHETIC JET FOR UNDERWATER FLOW CONTROL APPLICATIONS
}

\author{
A thesis submitted \\ in partial fulfillment of the requirements \\ for the degree of
}

Doctor of Philosophy

by

Abhay Kumar



to the

Department of Mechanical Engineering Indian Institute of Technology Kanpur

Kanpur, India

March 2015 



\title{
Department of Mechanical Engineering
}

\author{
Indian Institute of Technology Kanpur
}

\section{Certificate}

It is certified that the work contained in the thesis titled Synthetic Jet For Underwater Flow Control Applications by Abhay Kumar has been carried out under our supervision and that this work has not been submitted elsewhere for a degree.

Arun Kumar Saha

Department of Mechanical Engineering Indian Institute of Technology Kanpur Kanpur, 208016 (U.P.) India
P. K. Panigrahi

Department of Mechanical Engineering Indian Institute of Technology Kanpur Kanpur, 208016 (U.P.) India

March 2015 



\section{Dedicated to}

my mother Damyanti Devi,

father Rambalak Singh,

my wife Priynka,

and

daughters Arpita, Navya and son Ansh 



\section{Abstract}

The formation, propagation, and interaction of vortex rings have been the subject of various researchers due to its wide application in nature and day-to-day life. Some of the important applications of vortex rings are volcanic eruption, propulsion of aquatic creatures, sewerage outfalls. As an active device, synthetic jet with a train of large scale coherent structures and zero net mass flow have many practical and industrial applications in flow control.

A synthetic jet is generated using a simple cavity with a small orifice at one end and a deformable flexible diaphragm at the opposite end oscillating at different frequency and amplitude. It generates a series of vortex rings due to the changes in the cavity size with diaphragm oscillation. The vortex ring moves away from the orifice exit plane under the influence of self-induced velocity and thus synthesize a jet. Synthetic jet is also called zero net mass flux (ZNMF) system, the net mass transfer through the opening in one cycle is zero but the jet imparts a net transport of momentum to the surroundings.

In this work, the aim is to study the characteristics of synthetic jet for various orifice shapes, actuation frequencies and diaphragm displacements. A series of experiments were performed to understand the behavior of circular and rectangular shape synthetic jet in quiescent flow environment. To achieve this, a synthetic jet generation unit mounted on the sidewall of water tank is used. Further, a torpedo shape model with built-in circular synthetic jet has been designed and developed and the behavior of synthetic jet in crossflow has been investigated. For qualitative study, the bulk flow visualization and laser induced fluorescence (LIF) technique has been used whereas for quantitative measurement, the velocity is measured using hot-film anemometry and Laser Doppler Velocimetry (LDV).

Circular orifices of 8,10 and $13 \mathrm{~mm}$ diameter operating at actuation frequencies of 1, 2, 4 and $6 \mathrm{~Hz}$ have been used for characterization of circular synthetic jet generation. 
Non-circular orifices having same hydraulic diameter as that of circular one, i.e. square orifice and two rectangular shaped orifices with aspect ratios of 2 and 4 have been employed. The actuation frequencies of 1,2, 4 and $6 \mathrm{~Hz}$ have been used. The velocity characteristics in time-domain, frequency-domain and joint time-frequency domain are also examined for circular, square and rectangular orifices.

A torpedo shaped model with single built-in synthetic jet generation unit is fabricated to study the behavior of synthetic jet in crossflow inside a water tunnel. The actuation mechanism consists of a motor driven eccentric cam, which oscillates the diaphragm and generates synthetic jet. Initially, the synthetic jet is characterized for two different diaphragm displacements namely, $1.5 \mathrm{~mm}$ and $2 \mathrm{~mm}$ at four different actuation frequencies $(1,2,4$ and $6 \mathrm{~Hz})$ in quiescent flow environment. Subsequently, similar study is carried out in crossflow for three different crossflow velocities $\left(\mathrm{U}_{\infty}=0.072,0.2\right.$ and 0.32 $\mathrm{m} / \mathrm{s}$ ) at a single diaphragm displacement of $2 \mathrm{~mm}$.

The findings of the dissertation are organized along the following lines.

\section{Circular and rectangular orifice in quiescent environment:}

The spreading of circular synthetic jet is the highest for $8 \mathrm{~mm}$ diameter orifice and lowest for $10 \mathrm{~mm}$ diameter orifice beyond the vortex overshoot region $(\mathrm{X} / \mathrm{D}<3)$. Four distinct forms of synthetic jet namely: (i) partially formed synthetic jet, (ii) jet without trailing jet, (iii) jet with coherent trailing jet and (iv) jet with chaotic or turbulent trailing jet are observed depending on $\mathrm{L} / \mathrm{D}_{\mathrm{h}}$ and Reynolds number for synthetic jet with circular orifices.

The presence of corners in rectangular orifices significantly alters the vortex formation mechanism and its propagation. At certain distance downstream from the orifice exit, the flow pattern changes from laminar to chaotic or probably turbulent more rapidly than that of the circular orifices, operating at same actuation frequency. Depending on the $\mathrm{L} / \mathrm{D}_{\mathrm{h}}$ and 
Reynolds number, the vortex ring experiences axial switching and the jet reveals bifurcation for an aspect ratio $\mathrm{AR}=2$. At an aspect ratio $\mathrm{AR}=4$, the vortex rings break into multiple tiny rings close to the orifice exit. The coherent structures (vortex rings in the present study) represent themselves in the form of peaks evenly distributed on time axis. These peaks are observed at actuation frequency and its harmonics in frequency domain. The joint time-frequency representation indicates the distribution of total energy of the velocity signal in the form of ribs and bands. Synthetic jets having trailing jet are represented by bands parallel to the time axis at different harmonics. For chaotic/turbulent jet, first harmonic shares maximum amount of energy unlike laminar vortex rings where the energy is distributed at different harmonics.

\section{Synthetic jet in crossflow:}

The behavior of synthetic jet changes significantly due to influence of crossflow on the formation, convection, and interaction with neighboring vortices. The synthetic jet in crossflow has been investigated for various crossflow velocities $\left(\mathrm{U}_{\infty}=0.072,0.2\right.$ and 0.32 $\mathrm{m} / \mathrm{s})$ and different actuation frequencies $(2,4$ and $6 \mathrm{~Hz})$. The flow visualization results indicate that the vortex rings in synthetic jet can be classified into two major groups: (a) stretched vortex ring and (b) distorted tilted vortex ring.

The flow structures primarily depend on the velocity ratio, which is function of actuation frequency and crossflow velocity. At low velocity ratio below a value of 1.14 , the leading vortex is followed by strong trailing jet. Two different trajectories of vortices are observed at higher velocity ratio (3.18 and 4.77): one for the leading ones and the other for the trailing ones. At low crossflow velocity and higher actuation frequency, synthetic jet leads to increase in the velocity within the boundary layer in upstream side of the orifice. 
At higher velocity ratio, the core region of crossflow is affected by the synthetic jet. However, the flow near the wall is influenced only at lower velocity ratio. 


\section{Acknowledgements}

I take this opportunity to express my sincere thanks to my supervisor Dr. Arun K. Saha and Prof. P. K. Panigrahi for their guidance and support. I would also like to express my gratitude towards them for showing confidence in me by giving me a complete freedom to choose my own working path from topic selection to thesis completion. It was a privilege to have a great experience working under him in a cordial environment.

I am very much thankful to the Indian Institute of Technology Kanpur, for providing me the opportunity of purusing $\mathrm{PhD}$ in a peaceful environment with ample resources. I am also grateful to my institute for providing me the necessary financial support to present my work at several national and international conferences. I am very much thankful to Naval Research Board, New Delhi, Government of India for funding provided to establish the hydrodynamic research facility to conduct the experiment.

I am thankful to Shambhunath Sharma, Manoj Sharma, Sushil Mishra, Pulkit, Alok and Vimlesh for helping in fabrication work and facilitating me to avail the workshop facility without which the enormity of experiment done would have been impossible. I had the pleasure to share my time with my colleagues at Heat Transfer Lab and especially thanks to Sandesh, Madhu, Vivek, Yogang, and Manish for their significant contribution at different stages of experiment.

I would like to give special thanks to Trushar, Dhananjay, Narendra, Istiyak, and Rohit for their pleasant company and support. I am thankful to Abhishek, Asfer and Balkrishna for their boundless support and care throughout my stay at IIT Kanpur. All my friends especially Sumer, Wahadat, Shushil, Ramesh, Nirmal, Sachindananda, Bharti, Dhananjay Singh, Tapan, Sunil, Rakesh Murya, Atul Dhar, Akram, Umesh, Prasana and many others whom I am not able to remember due to my human shortcomings.

In the end, I would like to acknowledge my parents, wife, children, elder brother and his family. Without their support, this work would not have been possible. 


\section{Contents}

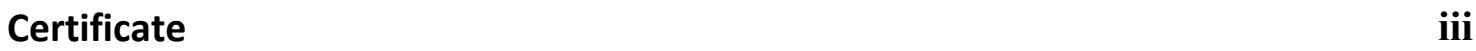

$\begin{array}{lll}\text { Abstract } & \text { vii }\end{array}$

Acknowledgements $\quad$ xi

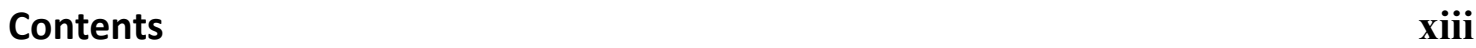

\begin{tabular}{ll} 
List of Figures & xix \\
\hline
\end{tabular}

List of Tables $\quad$ Xxxi

$\begin{array}{ll}\text { Nomenclature } & \text { xxxiii }\end{array}$

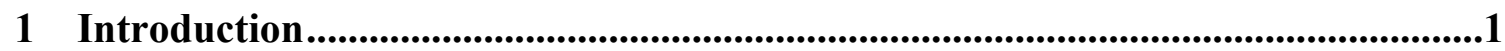

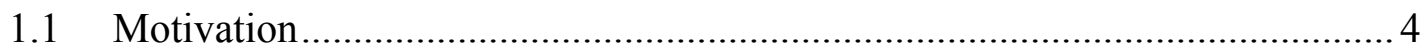

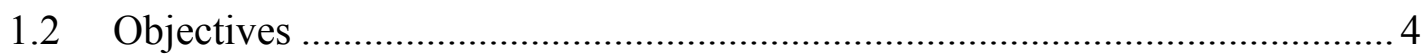

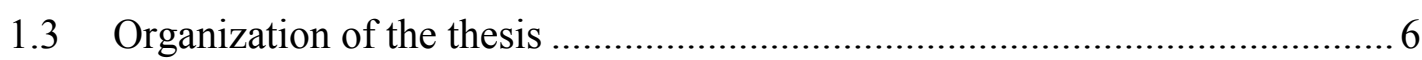

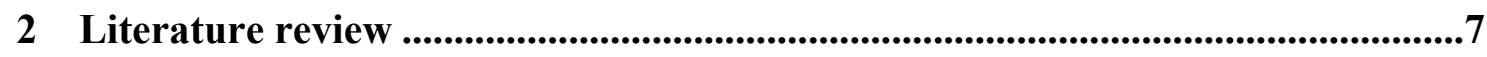

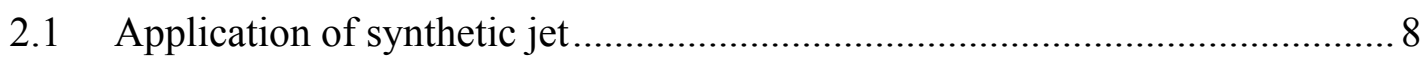



2.1.2 Spray technique and combustion control ............................................ 8

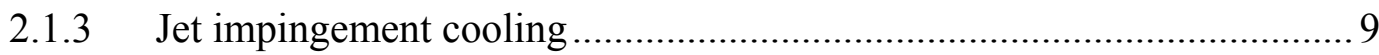

2.1.4 Boundary layer control .................................................................. 10

2.1.5 Propulsion and maneuvering................................................................. 11

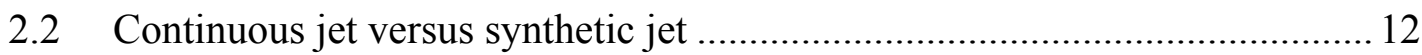

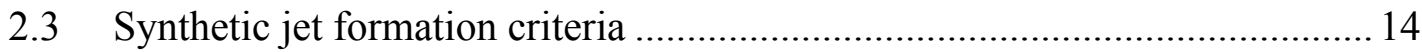

2.4 Parameters affecting the Synthetic jet formation....................................... 18 


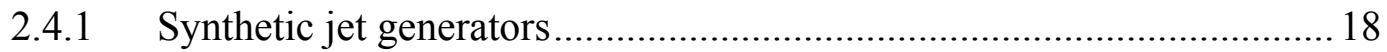

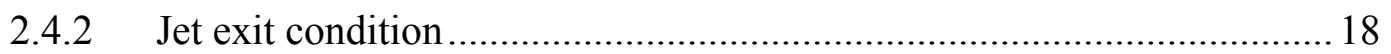

2.4.3 Cavity design and diaphragm location................................................ 19

2.4.4 Velocity program of actuator ............................................................ 19

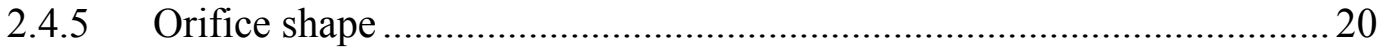

2.4.6 Actuation frequency and amplitude of diaphragm displacement ..........23

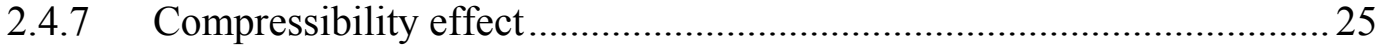

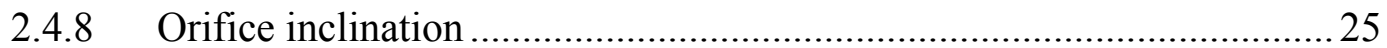

2.5 Synthetic jet behavior in non-quiescent flow condition ............................26

2.5.1 Synthetic jet in parallel-flow condition: ............................................... 27

2.5.2 Synthetic jet in crossflow condition.................................................... 28

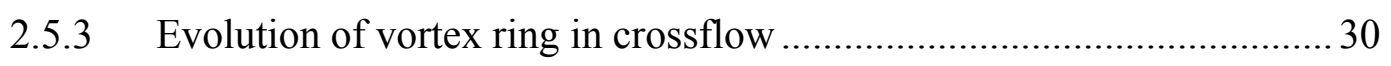



3 Experimental set-up and measurement techniques .......................................33

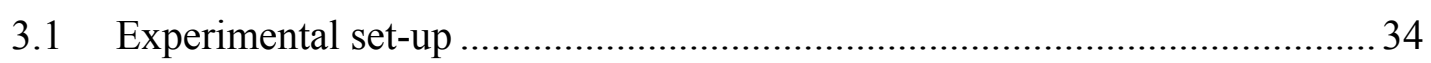

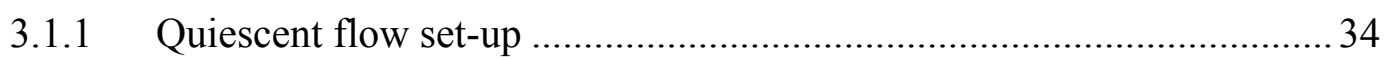

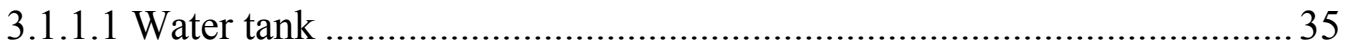

3.1.1.2 Synthetic jet cavity and orifice orientation........................................ 35





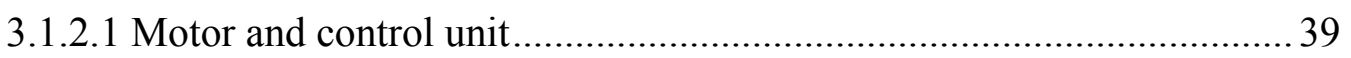

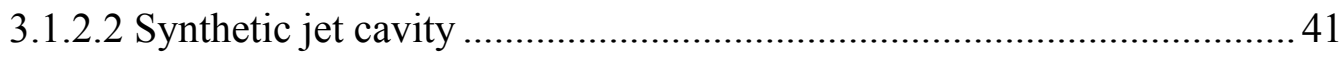

3.1.2.3 Diaphragm and actuation mechanism ............................................. 41

3.1.2.4 Assembly of components ............................................................. 42

3.1.2.5 Dynamic model support system ....................................................... 43

3.1.2.6 Actuation frequency and slug length................................................ 45

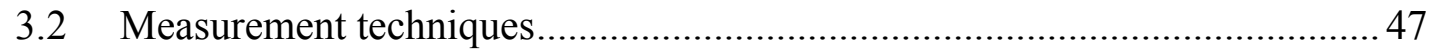



3.2.1.1 Experimental set-up for hot-film measurement.................................. 49

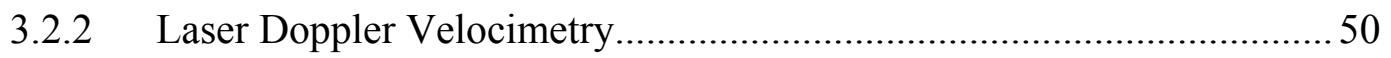


3.2.2.1 Measurement volume .51

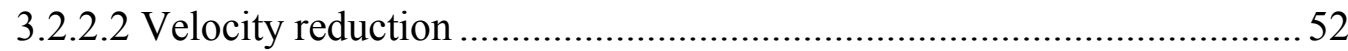

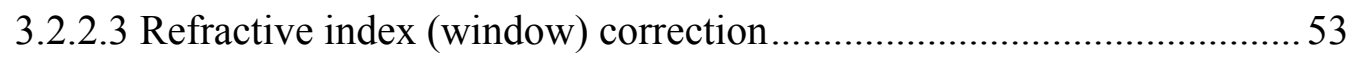

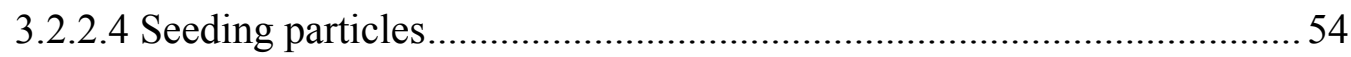

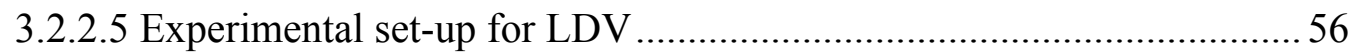



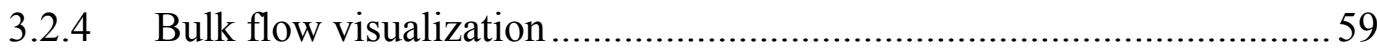

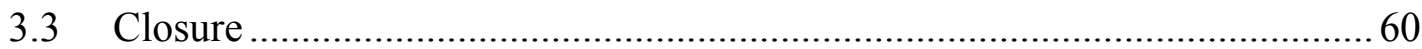

\section{Synthetic jet in quiescent flow: effect of orifice shape and excitation}

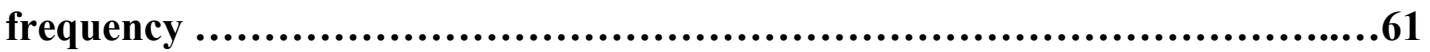

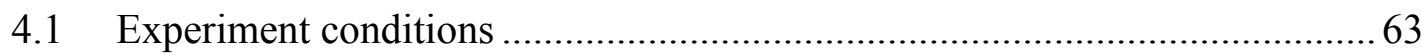

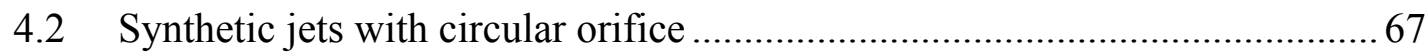



4.2.2 Time-averaged centerline velocity …................................................. 77

4.2.3 Time-averaged velocity field in transverse direction ........................... 80

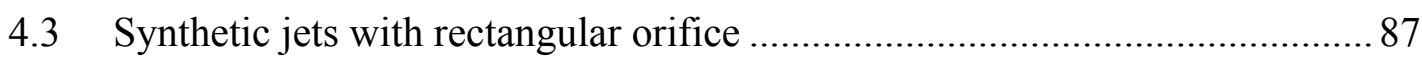



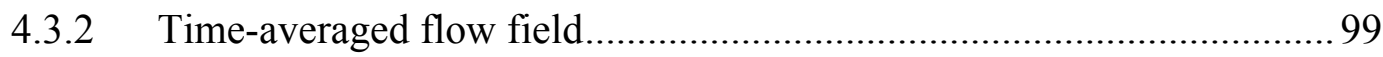

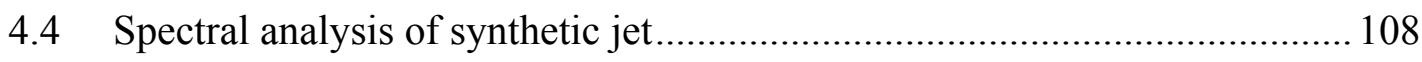

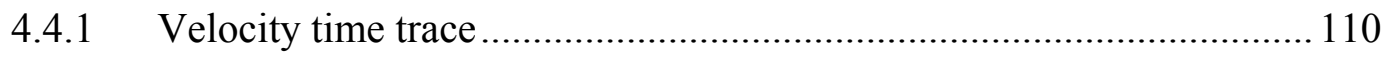

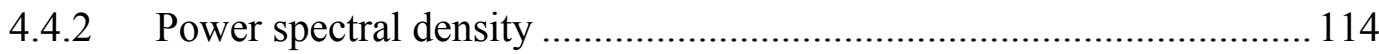

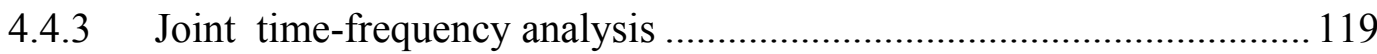

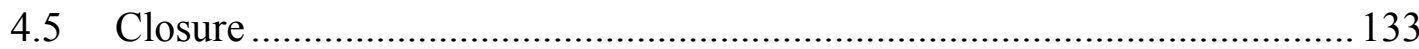

5 Bifurcation in synthetic jet with rectangular shape orifice.............................135

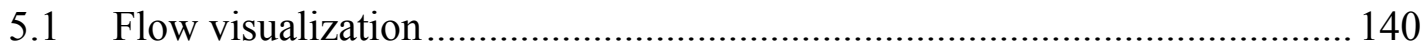

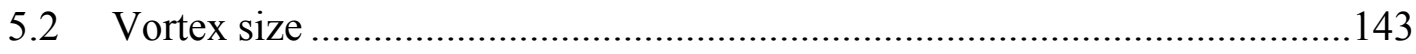

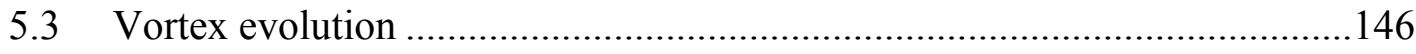




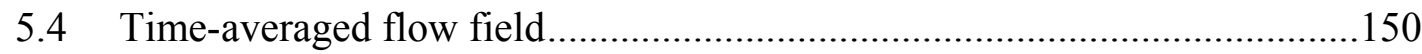

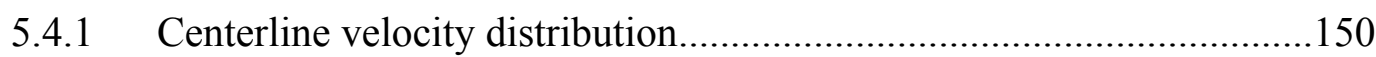

5.4.2 Velocity distribution in transverse directions ......................................151



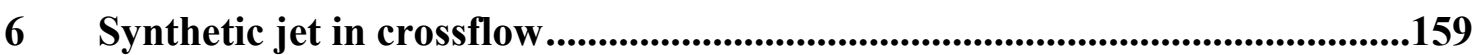

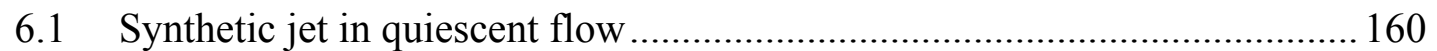

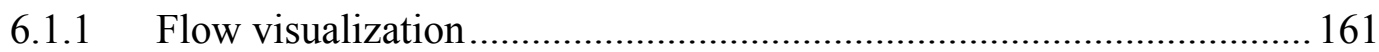



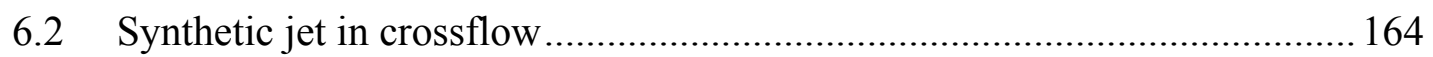



6.2.2 Time-averaged flow field.............................................................. 180

6.2.2.1 Velocity flow field with and without orifice on torpedo shape model.181

6.2.2.2 Effect of actuation frequency and velocity ratio

6.3 Closure

7 Conclusions and scope for future work ..........................................................191

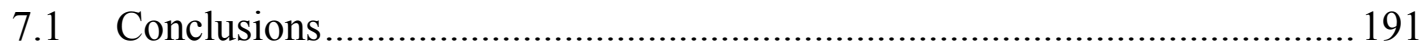

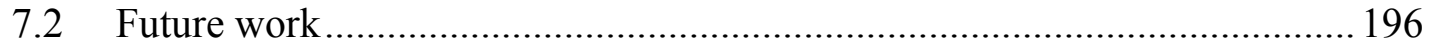

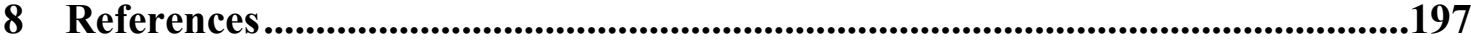

9 Appendix A. Vortex formation and effect of dimensionless numbers .............209

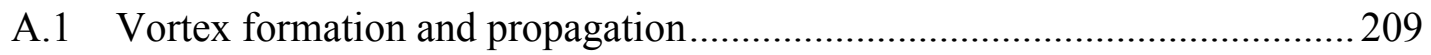

A.2 Dimensional analysis of synthetic jet .................................................. 212



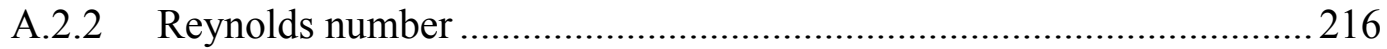

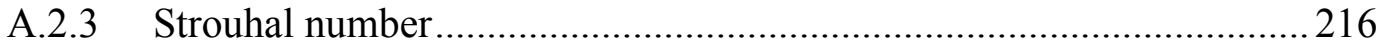

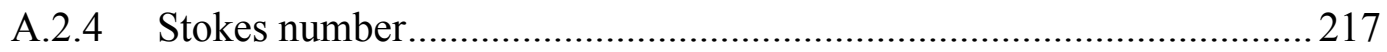




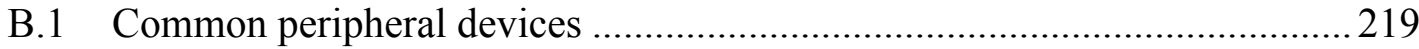

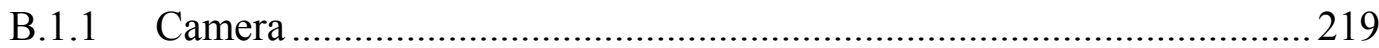

B.1.2 3-D Traverse for Hot film positioning ............................................... 220

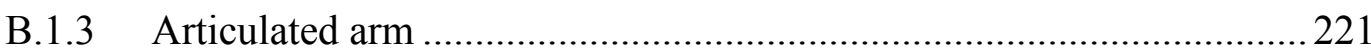

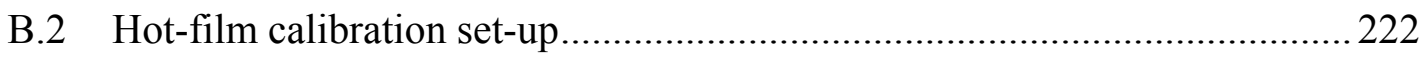

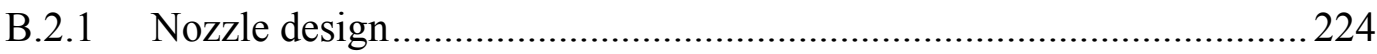

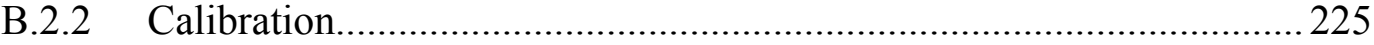

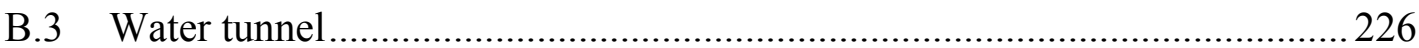

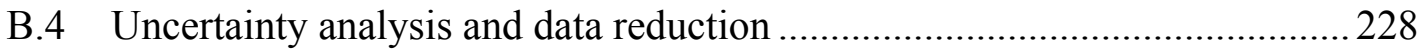

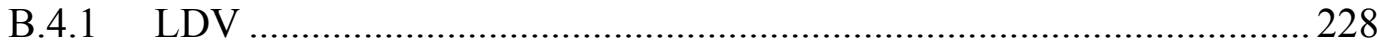

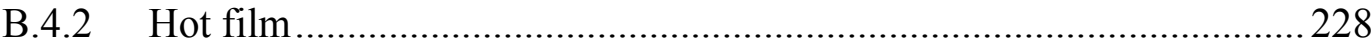

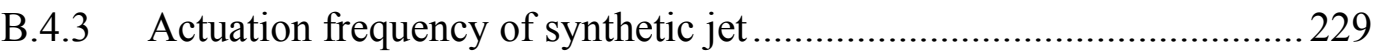



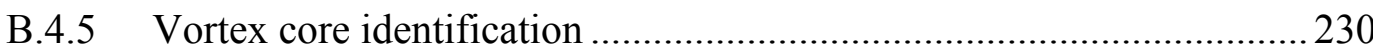




\section{List of figure}

Figure 1.1: Various applications of synthetic jet in fluid flow control...........................2

Figure 1.2: Formation of synthetic jet using diaphragm-orifice mechanism 3

Figure 2.1: Schlieren images of (a) continuous jet (b) oscillating jet and (c) synthetic jet for $\mathrm{b} / \mathrm{h}=17$ and $\mathrm{Re}=2200$. (Smith \& Swift, 2001)

Figure 2.2: (a) Mean streamwise velocity profile along the jet centerline and (b) half width of jet ' $b$ ' as a function of distance from injection nozzle. Nozzle width (e) and breadth are equal to $1 \mathrm{~mm}$ and $100 \mathrm{~mm}$ respectively. (Bera et al., 2001)

Figure 2.3: Images of vortex ring at three different $\mathrm{L} / \mathrm{D}$ ratio $(2,4$, and 6$)$ and distribution of average thrust with respect to L/D ratio (Gharib et al., 1998; Kruger, 2003)

Figure 2.4: Exit velocity distribution of (a) piston-cylinder mechanism and (b) diaphragm-orifice mechanism (Kruger, 2005).

Figure 2.5: Simulated pattern of axial switching (a) in planes perpendicular to synthetic jet centerline (YZ), (b) in major axis plane of orifice (XY) and (c) in minor axis plane (XZ) at different stages of vortex evolution (Kiya et al., 1992)...22

Figure 2.6: Flow field images of (a) Laminar jet at $\mathrm{Re}=3336, \mathrm{St}=0.3$, (b) Laminar rings at $\mathrm{Re}=7784, \mathrm{St}=0.012$, (c) Transitional jet at $\mathrm{Re}=11121, \mathrm{St}=0.009$ and (d) turbulent jet at $\mathrm{Re}=66991, \mathrm{St}=0.009$ (Cater and Soria, 2002)

Figure 2.7: Schematic of different types of vortices resulting from jet in crossflow (Kelso et al., 1996).

Figure 3.1: Orientation of synthetic jet and laser sheet in water tank for image acquisition in side view.

Figure 3.2: (a) Cross-sectional view of cavity, orifice and synthetic jet in XZ-plane, and (b) co-ordinate notation and orifice geometry details of synthetic jet 
Figure 3.3: (a) Synthetic jet actuator cavity with orifice plate, (b) top view of electromagnetic actuator connected to diaphragm, (c) power supply unit with frequency and power controller and (d) height adjustment and alignment base of electromagnetic actuator

Figure 3.4: Electromagnetic actuator power $(\mathrm{P})$ verses volume of fluid displaced (Q) by diaphragm during forward stroke.

Figure 3.5: Power supply, controller, power driver board and DC brushless motor for synthetic jet built-in in torpedo shaped model. 40

Figure 3.6: (a) Back view of diaphragm connected to cam follower, (b) front view of diaphragm with cavity and (c) pictorial representation of complete synthetic jet generation mechanism.

Figure 3.7: a) Exploded view of torpedo shape model with built-in synthetic jet, (b) model in test section of water tunnel and (c) assembled view of model

Figure 3.8: (a) Front and (b) back side view of dynamic model support system .44

Figure 3.9: (a) Actuation frequency obtained from pulse generated by sensors mounted at interval of 72 degree inside the motor and (b) flow frequency obtained from FFT of jet exit velocity. Plots are shifted in Y-axis for clarity

Figure 3.10: Schematic of hot-film calibration set-up

Figure 3.11: (a) Cross sectional view of measuring volume, (b) zoomed view of actual fringe and (c) burst signal when a particle crosses the fringe pattern. (*Figure (b) and (c) are taken from the TSI thermal manual).

Figure 3.12: Deviation in the path of laser beam due to changes in refractive index of optical medium.

Figure 3.13: Schematic of experimental set-up for Laser Doppler Velocimetry for (a) laser beam propagation inside the 5-beam probe gun and (b) front view of achromatic lens showing positions of all six beams. 
Figure 3.14: Schematic of experimental set-up used for laser induced fluorescence technique

Figure 4.1: Schematic representation of induced velocity of non-circular vortex tube. 62

Figure 4.2: Nomenclature for reporting the experimental results (a) circular orifice flow visualization plane, (b) rectangular orifice flow visualization planes, (c) orifice notations and (d) separation distance between the counter rotating vortices in plane $\mathrm{XZ}$ and $\mathrm{XY}$.

Figure 4.3: Flow visualization images of synthetic jet in XZ-plane (side view) for $8 \mathrm{~mm}$ (D8), 10mm (D10), and $13 \mathrm{~mm}$ (D13) circular shaped orifices at 1 and $2 \mathrm{~Hz}$ actuation frequency.

Figure 4.4: Flow visualization images of synthetic jet in XZ-plane (side view) for 8 $\mathrm{mm}$ (D8), $10 \mathrm{~mm}$ (D10), and $13 \mathrm{~mm}$ (D13) circular shaped orifices at 4 and $6 \mathrm{~Hz}$ actuation frequency

Figure 4.5: (a) Schematic of separation distance between the cores of counter rotating vortices in LIF images. The measured distance for (b) $8 \mathrm{~mm}$, (c) $10 \mathrm{~mm}$, and (d) $13 \mathrm{~mm}$ are shown at 1, 2, 4, and $6 \mathrm{~Hz}$ actuation frequency. (The fitted curves are shifted up and down by a value 0.5 for clarity.)

Figure 4.6: Various regions of time-averaged velocity profile along the synthetic jet centerline.

Figure 4.7: Time-averaged streamwise velocity measured along the synthetic jet centerline at different downstream locations actuating at 1, 2, 4 and $6 \mathrm{~Hz}$ for orifice diameter equal to (a) $8 \mathrm{~mm}$, (b) $10 \mathrm{~mm}$ and (c) $13 \mathrm{~mm}$. The hollow and solid symbols correspond to actual measurement using hot-film and corrected values by correcting the error of directional insensitiveness in hot-film.

Figure 4.8: Time-averaged streamwise velocity profile on non-dimensional and actual scale for $8 \mathrm{~mm}$ orifice in transverse direction at downstream locations $\mathrm{X} / \mathrm{D}$ $=2,5,8,12$ and 15 for actuation frequencies (a, i) $1 \mathrm{~Hz}$, (b, ii) $2 \mathrm{~Hz}$, (c, iii) $4 \mathrm{HZ}$ and (d, iv) $6 \mathrm{~Hz}$ 
Figure 4.9: Time-averaged streamwise velocity profile on non-dimensional and actual scale for $10 \mathrm{~mm}$ orifice in transverse direction at downstream locations $\mathrm{X} / \mathrm{D}$ $=2,5,8,12$ and 15 for actuation frequencies (a, i) $1 \mathrm{~Hz}$, (b, ii) $2 \mathrm{~Hz}$, (c, iii) $4 \mathrm{HZ}$ and (d, iv) $6 \mathrm{~Hz}$

Figure 4.10: Time-averaged streamwise velocity profile on non-dimensional and actual scale for $13 \mathrm{~mm}$ orifice in transverse direction at downstream locations X/D $=2,5,8,12$ and 15 for actuation frequencies (a, i) $1 \mathrm{~Hz}$, (b, ii) $2 \mathrm{~Hz}$, (c, iii) $4 \mathrm{HZ}$ and (d, iv) $6 \mathrm{~Hz}$..

Figure 4.11: Flow visualization images of synthetic jet in XZ-plane (side view) for square orifice $\left(A R 1\right.$ ) at (a) $f=1 \mathrm{~Hz}, \mathrm{~L} / \mathrm{D}_{\mathrm{h}}=1.6, \mathrm{Re}_{\mathrm{SJ}}=242$, (b) $\mathrm{f}=2 \mathrm{~Hz}, \mathrm{~L} / \mathrm{D}_{\mathrm{h}}$ $=2.3, \operatorname{ResJ}_{\mathrm{SJ}}=553,(\mathrm{c}) \mathrm{f}=4 \mathrm{~Hz}, \mathrm{~L} / \mathrm{D}_{\mathrm{h}}=3.3$, ResJ $=1742$, and (d) $\mathrm{f}=6 \mathrm{~Hz}, \mathrm{~L} /$ $\mathrm{D}_{\mathrm{h}}=4.5$, Ress $=3846$.

Figure 4.12: Flow visualization images of synthetic jet in XZ-plane (side view) and in XY-plane (top view) for rectangular shaped orifice (AR2) at ((a) $\&(i)) f=1 \mathrm{~Hz}$, $\mathrm{L} / \mathrm{D}_{\mathrm{h}}=1.5, \operatorname{Res}_{\mathrm{SJ}}=215$, ((b) \& (ii)) $\mathrm{f}=2 \mathrm{~Hz}, \mathrm{~L} / \mathrm{D}_{\mathrm{h}}=2.1, \operatorname{Re}_{\mathrm{SJ}}=491$, ((c) \& (iii)) at $\mathrm{f}=4 \mathrm{~Hz}, \mathrm{~L} / \mathrm{D}_{\mathrm{h}}=2.9$, ResJ $=1549$ and ((d) \& (iv)) $\mathrm{f}=6 \mathrm{~Hz}, \mathrm{~L} / \mathrm{D}_{\mathrm{h}}=4$, ResJ $=$ 3435. (*The images in XY and XZ planes are not at same time instant)....

Figure 4.13: Flow visualization images of synthetic jet in $\mathrm{XZ}$ plane (side view) and in XY-plane (top view) for rectangular shape orifice (AR4) at ((a) \& i)) $\mathrm{f}=1 \mathrm{~Hz}$, $\mathrm{L} / \mathrm{D}_{\mathrm{h}}=1.1, \operatorname{Re}_{\mathrm{SJ}}=155$, ((b) \& ( ii)) $\mathrm{f}=2 \mathrm{~Hz}, \mathrm{~L} / \mathrm{D}_{\mathrm{h}}=1.5$, ResJ $=354$, ((c) \& (iii)) at $\mathrm{f}=4 \mathrm{~Hz}, \mathrm{~L} / \mathrm{Dh}_{\mathrm{h}}=2.1$, Ress $=1115$ and ((d) \& (iv)) $\mathrm{f}=6 \mathrm{~Hz}, \mathrm{~L} / \mathrm{Dh}_{\mathrm{h}}=2.9$, Res $=2473$. $(*$ The images in $\mathrm{XY}$ and $\mathrm{XZ}$ planes are not at same time instant).....

Figure 4.14: (a) Stream wise velocity of front end of leading vortex ring $\left(U_{f}\right)$ and counter rotating vortices of leading vortex ring $\left(U_{r}\right)$ calculated from the sequence of LIF images for AR1 and AR2 orifices in XZ-plane for (b) $1 \mathrm{~Hz}$, (c) $2 \mathrm{~Hz}$, (d) 4 $\mathrm{Hz}$ and (e) $6 \mathrm{~Hz}$ actuation frequency

Figure 4.15: Schematic of the counter rotating vortices in major (XY) and minor (XZ) axis planes for non-circular vortex ring. 96 
Figure 4.16: Separation distance between the counter rotating vortices in LIF images captured in XY and XZ-plane at actuation frequency 1, 2, 4 and $6 \mathrm{~Hz}$ for (a) AR1 (b) AR2 and (c) AR4 orifice cases.

Figure 4.17: Time-averaged streamwise velocity measured along the synthetic jet centerline at different downstream locations actuating at 1,2, 4 and $6 \mathrm{~Hz}$ actuation frequency for rectangular shaped orifices: (a) AR1, (b) AR2 and (c) AR4. (*The hollow and solid symbols correspond to actual measurement using hot film and corrected values by correcting the error of directional insensitiveness in hot film)..

Figure 4.18: Time-averaged streamwise velocity profile in transverse direction (Yaxis) at different downstream locations $\mathrm{X} / \mathrm{W}=1,3,5$ and 10 at (a) $1 \mathrm{~Hz}$, (b) $2 \mathrm{~Hz}$, (c) $4 \mathrm{~Hz}$ and (d) $6 \mathrm{~Hz}$ actuation frequency for square orifice (AR1). 103

Figure 4.19: Time-averaged streamwise velocity profile at different downstream locations $(\mathrm{X} / \mathrm{W}=1,3,5$ and 10) in cross-stream directions $\mathrm{Y}$ and $\mathrm{Z}$ for rectangular orifice (AR2) at different actuation frequencies: (a) $1 \mathrm{~Hz}$, (b) $2 \mathrm{~Hz}$, (c) $4 \mathrm{~Hz}$ and (d) $6 \mathrm{~Hz}$ 106

Figure 4.20: Time-averaged streamwise velocity profile at different downstream locations $(\mathrm{X} / \mathrm{W}=1,3,5$ and 10) in transverse directions $\mathrm{Y}$ and $\mathrm{Z}$ for rectangular orifice (AR4) at different actuation frequencies : (a) $1 \mathrm{~Hz}$, (b) $2 \mathrm{~Hz}$, (c) $4 \mathrm{~Hz}$ and (d) $6 \mathrm{~Hz}$ 107

Figure 4.21: Flow visualization images of synthetic jet in XZ-plane (side view) for circular orifice (D10), square orifice (AR1), rectangular orifice of aspect ratio two (AR2) and for rectangular orifice of aspect ratio four (AR4) at ((a), (b), (c) and (d)) $1 \mathrm{~Hz}$ actuation frequency and ((i), (ii), (iii) and (iv)) $6 \mathrm{~Hz}$ actuation frequency. (*The dots represent the location for S-type hot-film measurement carry out spectral analysis. It may be noted that images are not at same phase). 109

Figure 4.22: Velocity time trace of synthetic jet using hot-film along the synthetic jet centerline at different downstream locations for circular orifice (D10), square orifice (AR1), rectangular orifice of aspect ratio two (AR2) and rectangular orifice of aspect ratio 4 (AR4) at $1 \mathrm{~Hz}$ actuation frequency (a-d), $6 \mathrm{~Hz}$ actuation frequency 
(e-h) and $18 \mathrm{~Hz}$ actuation frequency (i-1). (* The plots are shifted upward by a step size of 4 or 5 for clarity)

Figure 4.23: Power spectral density (PSD) distribution along the centerline at $6 \mathrm{~Hz}$ actuation frequency for $10 \mathrm{~mm}$ circular orifice $(\mathrm{D} 10)$ at $\mathrm{X} / \mathrm{D}=3$.

Figure 4.24: Power spectral density (PSD) of streamwise velocity along synthetic jet centerline at different downstream locations for circular orifice (D10), square orifice (AR1), rectangular orifice (AR2) and rectangular orifice (AR4) at (a-d) 1 $\mathrm{Hz}$, (e-h) $6 \mathrm{~Hz}$ and (i-1) $18 \mathrm{~Hz}$ actuation frequency 116

Figure 4.25: Magnitude of power spectral density at first harmonic along the synthetic jet centerline for (a) D10, (b) AR1, (c) AR2 and (d) AR4 orifices at 1,6 and $18 \mathrm{~Hz}$ actuation. (*The values of $\mathrm{PSD}_{\mathrm{f}}$ more than $10 \%$ of maximum achieved value at first harmonic are represented by dark symbols and the value less than $10 \%$ are represented by shaded symbols).

Figure 4.26: (a) Fast Fourier transformation and (b) Winger-Valley distribution of velocity signal at $18 \mathrm{~Hz}$ actuation frequency orifice at $\mathrm{X} / \mathrm{D}=5$. (c) Representation of velocity signal at $6 \mathrm{~Hz}$ actuation frequency at $\mathrm{X} / \mathrm{D}=5$ in time, frequency and joint time-frequency domain with (d) extracted value of energy form TimeFrequency representation plot at integer multiple of actuating frequency or at harmonic frequency. (e) 3-Dimendional view of joint time frequency representation of $\mathrm{D} 10$ orifice for $6 \mathrm{~Hz}$ actuation frequency at $\mathrm{X} / \mathrm{D}=5$.

Figure 4.27: Choi-William transformation at $1 \mathrm{~Hz}$ actuation frequency in downstream direction along the synthetic jet centerline at three different stream wise locations $\mathrm{X} / \mathrm{D}$ or $\mathrm{X} / \mathrm{W}=3,5$ and 8 for circular orifice (D10) are shown in ((a), (b) \& (c))and for square orifice(AR1) are shown in ((i), (ii) \& (iii)).

Figure 4.28: Choi-William transformation at $1 \mathrm{~Hz}$ actuation frequency in downstream direction along the synthetic jet centerline at three different stream wise locations $\mathrm{X} / \mathrm{W}=3,5$ and 8 for rectangular orifice (AR2) are shown in ((a), (b) \& (c))and for rectangular orifice(AR4) are shown in ((i), (ii) \& (iii)). 128 
Figure 4.29: Choi-William transformation at $6 \mathrm{~Hz}$ actuation frequency in downstream direction along the synthetic jet centerline at three different stream wise locations $\mathrm{X} / \mathrm{D}$ or $\mathrm{X} / \mathrm{W}=3,8$ and 12 for circular orifice (D10) are shown in ((a), (b) \& (c))and for square orifice(AR1) are shown in ((i), (ii) \& (iii)).

Figure 4.30: Choi-William transformation at $6 \mathrm{~Hz}$ actuation frequency in downstream direction along the synthetic jet centerline at three different stream wise locations $\mathrm{X} / \mathrm{W}=3,8$ and 12 for rectangular orifice (AR2) are shown in ((a), (b) \& (c))and for rectangular orifice(AR4) are shown in ((i), (ii) \& (iii)). 130

Figure 4.31: Choi-William transformation at $18 \mathrm{~Hz}$ actuation frequency in downstream direction along the synthetic jet centerline at three different stream wise locations $\mathrm{X} / \mathrm{D}$ or $\mathrm{X} / \mathrm{W}=3,8$ and 12 for circular orifice (D10) are shown in ((a), (b), (c))and for square orifice(AR1) are shown in ((i), (ii), (iii))

Figure 4.32: Choi-William transformation at $18 \mathrm{~Hz}$ actuation frequency in downstream direction along the synthetic jet centerline at three different stream wise locations $\mathrm{X} / \mathrm{W}=3,8$ and 12 for rectangular orifice (AR2) are shown in ((a), (b) \& (c))and for rectangular orifice(AR4) are shown in ((i), (ii) \& (iii)). 132

Figure 5.1: Schematic of (a) orifice orientation and (b) axis notations

Figure 5.2: Flow visualization images of synthetic jet in $X Z$-plane (side view) and in $\mathrm{XY}$-plane (top view) for bifurcation cases (a) Case 1: $\mathrm{f}=1 \mathrm{~Hz}, \mathrm{Re}=275, \mathrm{~L} / \mathrm{D}_{\mathrm{h}}=$ 1.51, (b) Case II: $\mathrm{f}=2 \mathrm{~Hz}, \mathrm{Re}=1101, \mathrm{~L} / \mathrm{D}_{\mathrm{h}}=3.832 \mathrm{~Hz}$ and (c) Case III: $\mathrm{f}=3$ $\mathrm{Hz}, \mathrm{Re}=1309, \mathrm{~L} / \mathrm{D}_{\mathrm{h}}=2.94$. ( ${ }^{*}$ It may be noted that images are not exactly at same instant of time)

Figure 5.3: Flow visualization images of synthetic jet in XZ-plane (side view) and in $\mathrm{XY}$-plane (top view) for non- bifurcation cases at (a) Case IV: $\mathrm{f}=1 \mathrm{~Hz}, \mathrm{Re}=625$ , $\mathrm{L} / \mathrm{D}_{\mathrm{h}}=3.42$, (b) Case V: $\mathrm{f}=2 \mathrm{~Hz}, \mathrm{Re}=1348, \mathrm{~L} / \mathrm{D}_{\mathrm{h}}=4.69$ and (c) Case VI: $\mathrm{f}=$ $3 \mathrm{~Hz}, \mathrm{Re}=2038, \mathrm{~L} / \mathrm{D}_{\mathrm{h}}=4.57$. (* It may be noted that images are not exactly at same instant of time).

Figure 5.4: Separation distance ((a) and (c)) between the cores of counter rotating vortices in time domain and position of cores ((b) and (d)) in space domain 
measured from the sequency of LIF images in XZ \& XY-plane for bifurcation cases (I, II and III) and for non-bifurcation cases (IV, V and VI). (*The values of $\mathrm{H}_{\mathrm{B}}$ and $\mathrm{Hw}$ are shifted in $\mathrm{Y}$ axis by step size equal to 2 from figure (c) for calarity).

Figure 5.5: Sequence of LIF images showing vortex evolution and bifurcation process at different time stamps in XZ-plane for Case I.

Figure 5.6: Sequence of LIF images showing vortex evolution and bifurcation process at different time stamps in XY-plane for Case I.

Figure 5.7: A schematic comparing the process (a) axial switching and (b) bifurcation in rectangular synthetic jet.

Figure 5.8: Time-averaged (a) streamwise velocity $\left(U_{\text {avg }}\right)$ and (b) cross-stream velocity ( $\mathrm{V}_{\text {avg }}$ ) along the synthetic jet centerline for bifurcation cases ((I), (II) and (III)) and for non-bifurcation cases ((IV), (V) and (VI)).

Figure 5.9: Time-averaged streamwise velocity $\left(U_{a v g}\right)$ profile normalised by $U_{o}$ for (a) Case I, (b) Case II and (c) Case III in Y-direction and (i) Case I, (ii) Case II and (iii) Case III in Z-direction at four downstream locations $(\mathrm{X} / \mathrm{W}=1,2,3$ and $5)$.

Figure 5.10: Time-averaged streamwise velocity $\left(\mathrm{U}_{\mathrm{avg}}\right)$ profile normalized by $\mathrm{U}_{\mathrm{o}}$ for (a) Case VI, (b) Case V and (c) Case VI in Y-direction and (i) Case VI, (ii) Case $\mathrm{V}$ and (iii) Case VI in Z-direction at four downstream locations $(\mathrm{X} / \mathrm{W}=1,2,3$ and $5)$.

Figure 5.11: Time-averaged cross-stream velocity $\left(\mathrm{V}_{\text {avg }}\right)$ profile normalised by $\mathrm{U}_{\mathrm{o}}$ for (a) Case I, (b) Case II and (c) Case III in Y-direction and (i) Case I, (ii) Case II and (iii) Case III in Z-direction at four downstream locations (X/W = 1, 2, 3 and 5)

Figure 5.12: Time-averaged cross-stream velocity $\left(\mathrm{V}_{\text {avg }}\right)$ profile normalised by $\mathrm{U}_{\mathrm{o}}$ for (a) Case III, (b) Case V and (c) Case VI in Y-direction and (i) Case III, (ii) Case V and (iii) Case VI in Z-direction at four downstream locations (X/W =1, 2, 3 and 5) 
Figure 5.13: The range of Reynolds number for bifurcation observed in flow visualization images of synthetic jet of aspect ratio 2

Figure 6.1: Schematic for axis and orifice orientation with imaging plane for torpedo shape model (a) in quiescent flow and (b) in cross flow

Figure 6.2: Sequence of LIF images at $\mathrm{t} / \mathrm{T} \sim 0.75$ for (a) and (b) at $1 \mathrm{~Hz}$, (c) and (d) at $2 \mathrm{~Hz},(\mathrm{e})$ and (f) at $4 \mathrm{~Hz},(\mathrm{~g})$ and (h) at $6 \mathrm{~Hz}$ of actuation frequency for diaphragm displacement $(\Delta)$ of $1.5 \mathrm{~mm}$ and $2 \mathrm{~mm}$ respectively.

Figure 6.3: Time-averaged streamwise velocity profile (a) and (b) and cross-stream velocity profile (c) and (d) for $\Delta=1.5 \mathrm{~mm}$ and $\Delta=2 \mathrm{~mm}$ respectively along the synthetic jet centerline

Figure 6.4: Bulk flow visualization of synthetic jet fin side view at (a) $2 \mathrm{~Hz}$, (b) $4 \mathrm{~Hz}$ , and (c) $6 \mathrm{~Hz}$ of actuation frequency at $\left(\mathrm{U}_{\infty}\right)=0$. 166

Figure 6.5: Bulk flow visualization of synthetic jet from side and top view (a) 0.072 $\mathrm{m} / \mathrm{s}$ (b) $0.2 \mathrm{~m} / \mathrm{s}$ and (c) $0.32 \mathrm{~m} / \mathrm{s}$ are shown for actuation frequency of $2 \mathrm{~Hz}$ The dashed line is approximate boundary layer thickness without synthetic jet. 170

Figure 6.6: Bulk flow visualization of synthetic jet from side and top at crossflow velocity(a) $0.072 \mathrm{~m} / \mathrm{s}$ (b) $0.2 \mathrm{~m} / \mathrm{s}$ and (c) $0.32 \mathrm{~m} / \mathrm{s}$ are shown for actuation frequency of $4 \mathrm{~Hz}$ (*The dashed line is approximate boundary layer thickness with synthetic jet at $0 \mathrm{~Hz}$ actuation frequency).

Figure 6.7: Bulk flow visualization of synthetic jet from side and top (a) $0.072 \mathrm{~m} / \mathrm{s}$ (b) $0.2 \mathrm{~m} / \mathrm{s}$ and (c) $0.32 \mathrm{~m} / \mathrm{s}$ are shown for actuation frequency of $6 \mathrm{~Hz}$ (*The dashed line is approximate boundary layer thickness without synthetic jet). 172

Figure 6.8: At cross flow velocity $0.072 \mathrm{~m} / \mathrm{s}, 0.2 \mathrm{~m} / \mathrm{s}$ and $0.32 \mathrm{~m} / \mathrm{s}$, the sequence of LIF images at actuation frequency 2, 4, and $6 \mathrm{~Hz}$ are arranged in ascending order of velocity ratio. The images positioned at time stamp of $\mathrm{t} / \mathrm{T} \sim 0.25, \mathrm{t} / \mathrm{T} \sim 0.5, \mathrm{t} / \mathrm{T}$ $\sim 0.75$ and $\mathrm{t} / \mathrm{T} \sim 1$ 
Figure 6.9: Schematic representing the synthetic jet in cross flow having (a) hairpin and stretched leading vortex ring and (b) tilted and distorted vortex ring with stretched trailing jet.

Figure 6.10: (a) Evolution of synthetic jet in cross flow 180

Figure 6.11: (a) Grid points for LDV measurement for comparing the torpedo shape model with synthetic jet orifice at $0 \mathrm{~Hz}$ actuation frequency and without the presence of crossflow and (b) grid points for velocity measurement in crossflow for the conditions shown in Table 6.2

Figure 6.12: Velocity contours over the torpedo shape model with and without the cavity of synthetic jet(SJ) in test section of water tunnel at $0.2 \mathrm{~m} / \mathrm{s}$. (a) and (b) are the velocity component in cross stream directions and (c) and (d) are velocity component in synthetic jet direction.

Figure 6.13: Time-averaged velocity (W) parallel to crossflow at eight different locations $\mathrm{Z} / \mathrm{D}=-1,0,1,2,3,5,8$ and 12 for $0,2,4$ and $6 \mathrm{~Hz}$ actuation frequency at $\mathrm{U}_{\infty}=0.072 \mathrm{~m} / \mathrm{s}$. (For clarity velocity lines are shifted on $\mathrm{X}$-axis by a step size equal to one)

Figure 6.14: Time-averaged velocity (U) parallel to crossflow at eight different locations $\mathrm{Z} / \mathrm{D}=-1,0,1,2,3,5,8$ and 12 at $0,2,4$ and $6 \mathrm{~Hz}$ actuation frequency at $\mathrm{U}_{\infty}=0.072 \mathrm{~m} / \mathrm{s}$

Figure 6.15: Time-averaged velocity (W) parallel to crossflow at eight different locations $\mathrm{Z} / \mathrm{D}=-1,0,1,2,3,5,8$ and 12 at $0,2,4$ and $6 \mathrm{~Hz}$ actuation frequency at $\mathrm{U}_{\infty}=0.2 \mathrm{~m} / \mathrm{s}$. (For clarity velocity lines are shifted on $\mathrm{X}$-axis by a step size equal to one).

Figure 6.16: Time-averaged velocity (U) parallel to crossflow at eight different locations $\mathrm{Z} / \mathrm{D}=-1,0,1,2,3,5,8$ and 12 at 0,2 , and $6 \mathrm{~Hz}$ actuation frequency at $\mathrm{U}_{\infty}=0.2 \mathrm{~m} / \mathrm{s}$.

Figure 6.17: Time-averaged velocity (W) parallel to crossflow at eight different locations $\mathrm{Z} / \mathrm{D}=-1,0,1,2,3,5,8$ and 12 at $0,2,4$ and $6 \mathrm{~Hz}$ actuation frequency 
at $\mathrm{U}_{\infty}=0.32 \mathrm{~m} / \mathrm{s}$. (For clarity velocity lines are shifted on $\mathrm{X}$-axis by a step size equal to one. 188

Figure 6.18: Time-averaged velocity (U) parallel to crossflow at eight different locations $\mathrm{Z} / \mathrm{D}=-1,0,1,2,3,5,8$ and 12 at $0,2,4$ and $6 \mathrm{~Hz}$ actuation frequency at $\mathrm{U}_{\infty}=0.32 \mathrm{~m} / \mathrm{s}$. (For clarity velocity lines are shifted on $\mathrm{X}$-axis by a step size equal to one). 188

Figure A.1: (a) Shear layer roll up at nozzle jet exit during expulsion period , (b) flow of ambient fluid inside the cavity by induced flow during vortex detachment process (Maxworthy, 1997) and (c) the streamline distribution for an ideal isolated vortex ring (Akhmetov \&. Kisarov, 1966)

Figure A.2: (a) Velocity due to rolling of vortex rings (b) mass distribution for an isolated vortex ring (Krueger \& Gharib, 2003) and (c) mass distribution of a synthetic jet (Yu et al., 2007)....

Figure A.3: Slug model assumptions in vortex formation 215

Figure A.4: Effect of Reynolds number at three different value of slug length (Crook \& Wood, 2001)

Figure A.5: Effect of Stokes number on vortex formation at (a) $S=7$, (b) $S=12.1$ and (c) $\mathrm{S}=22.2$ having dimensionless stroke length (L/D) equal to 3.24 (Zhou et. al., 2009).

Figure B.1: Basler ${ }^{\circledR} 501 \mathrm{~K}$ CMOS camera

Figure B.2: (a) Design sketch and (b) actual image of 3-Dimensional traverse for mounting hot-film sensors 220

Figure B.3: Laser sheet with articulated light arm, laser source and its optics 221

Figure B.4: (a) Side and (b) top cross-sectional view of calibration set-up 223

Figure B.5: (a) potential core of jet and (b) contour profile of nozzle .224 
Figure B.6: (a) Hot film calibration curve, (b) cross-stream velocity profile of stream wise velocity at $0.5 \mathrm{D}$ from jet exit and (c) corresponding turbulence intensity of stream wise velocity at $0.5 \mathrm{D}$ from jet exit

Figure B.7: (a) 3D model and (b) actual view of water tunnel .227 


\section{List of Tables}

S. No.

4.1 Geometrical and experimental condition details for experiment with circular orifice

4.2 Geometrical and experimental conditions details for experiment with for rectangular orifice

4.3 Geometrical and experimental condition details for experiment on Spectral analysis

4.4 Geometrical and experimental condition details for experiment on Spectral analysis

4.5 Values of maximum streamwise velocity component at cross stream location $\left(U_{c}\right)$ in $\mathrm{m} / \mathrm{s}$ for AR2 orifice

4.6 Values of maximum streamwise velocity component at cross stream location $\left(U_{c}\right)$ in $\mathrm{m} / \mathrm{s}$ for $A R 4$ orifice

5.1 Details of experimental variables

6.1 Experimental parameters to study the synthetic jet in quiescent flow

6.2 Experimental parameters to study the synthetic jet in crossflow 


\section{Nomenclature}

\section{Roman Symbols}

\begin{tabular}{|c|c|}
\hline Ao & Cross-sectional area of orifice \\
\hline $\mathrm{B}$ & Breadth of orifice \\
\hline $\mathrm{C}_{1}$ & Shape constant of orifice \\
\hline $\mathrm{D}$ & Diameter of orifice \\
\hline $\mathrm{dA}$ & Elemental area \\
\hline $\mathrm{d}_{\mathrm{a}}$ & Particle aerodynamic diameter \\
\hline$d_{f}$ & Fringe spacing \\
\hline$d_{g}$ & Geometrical diameter of particle \\
\hline $\mathrm{d}_{\mathrm{m}}$ & Waist diameter of oval shape measuring volume \\
\hline $\mathrm{D}_{\mathrm{ca}}$ & Inner diameter of cavity \\
\hline $\mathrm{D}_{\mathrm{cy}}$ & Diameter of disk pasted on diaphragm center \\
\hline $\mathrm{D}_{\mathrm{h}}$ & Hydraulic diameter of rectangular orifice \\
\hline $\mathrm{E}$ & Bridge voltage of hot film \\
\hline $\mathrm{f}$ & Actuation frequency of synthetic jet \\
\hline $\mathrm{F}$ & Apparent focal length of lens \\
\hline$f_{B}$ & Braggs frequency \\
\hline $\mathrm{fD}_{\mathrm{D}}$ & Doppler frequency \\
\hline$f_{R}$ & Resultant frequency \\
\hline $\mathrm{F}_{\mathrm{D}}$ & Focal length of lens \\
\hline $\mathrm{H}$ & Height of synthetic jet cavity \\
\hline $\mathrm{h}$ & Thickness of orifice \\
\hline Нв & $\begin{array}{l}\text { Separation distance between the counter rotating vortices in LIF } \\
\text { images in major axis plane of orifice }\end{array}$ \\
\hline $\mathrm{H}_{\mathrm{D}}$ & $\begin{array}{l}\text { Separation distance between the counter rotating vortices in LIF } \\
\text { images }\end{array}$ \\
\hline $\mathrm{Hw}$ & $\begin{array}{l}\text { Separation distance between the counter rotating vortices in LIF } \\
\text { images in minor axis plane of orifice }\end{array}$ \\
\hline I & Ampere given to hot-film \\
\hline $\operatorname{lm}$ & Length of oval shape measuring volume \\
\hline
\end{tabular}


L Equivalent slug length of ejected fluid in expulsion stroke of diaphragm

Mo Momentum of synthetic jet

$\mathrm{P} \quad$ Pressure at a point

Qv Volume displaced by diaphragm during forward stroke of actuation

Radius of vortex tube

R, a Radius

$\operatorname{Re}$

Reynolds number

ResJ

Reynolds number of synthetic jet at $\mathrm{U}_{\infty}=0$

$\mathrm{R}_{\mathrm{w}}$

Resistance of hot film

$\mathrm{S}$

Stokes number

$\mathrm{St}$

Strouhal number

$\mathrm{St}_{\delta}$

Strouhal number

$\mathrm{t}$

Time

$\mathrm{T}$

Actuation cycle period of synthetic jet

$\mathrm{T}_{\mathrm{a}}$

Ambient temperature

$\mathrm{T}_{\mathrm{e}}$

Time period of diaphragm in ejection stroke

$\mathrm{T}_{\mathrm{s}}$

Time period of diaphragm in suction stroke

$\mathrm{T}_{\mathrm{w}}$

Water temperature

$u_{i}(t)$

Instantaneous velocity

$\mathrm{U}, \mathrm{V}, \mathrm{W}$

Velocity in direction of $\mathrm{X}, \mathrm{Y}$ and $\mathrm{Z}$ direction

$\mathrm{U}_{\mathrm{r}}$

Velocity of counter rotating vortices measured from sequence of LIF images

$\mathrm{Uf}_{\mathrm{f}} \quad$ Velocity of front end of vortex ring measured from sequence of LIF images

$\mathrm{U}_{\text {avg, }}, \mathrm{V}_{\text {avg, }} \quad$ Averaged velocities in $\mathrm{X}$ and $\mathrm{Y}$ directions

$\mathrm{U}_{\mathrm{C}}$

Maximum velocity of synthetic jet in transverse direction at particular downstream location

$\mathrm{U}_{\infty} \quad$ Crossflow velocity

$\mathrm{U}_{\mathrm{a}} \quad$ Averaged exit velocity of expelled fluid during forward stroke of diaphragm

VD Total volume of expelled fluid during forward stroke of diaphragm

$\mathrm{V}_{\text {induced }} \quad$ Induced velocity of vortex ring 
VR Velocity ratio

W Width of rectangular orifice

$\mathrm{X}, \mathrm{Y}, \mathrm{Z} \quad$ Cartesian coordinates

\section{Greek Symbols}

$\begin{array}{ll}\alpha & \text { Cross term suppression coefficient } \\ \beta & \text { Half angle } \\ \delta & \text { Boundary Layer thickness } \\ \mu & \text { Dynamic viscosity } \\ \varphi & \text { Phase difference in velocity signal } \\ \rho & \text { Fluid density } \\ \kappa & \text { Half angle in of laser beam in five beam LDV probe } \\ \lambda & \text { Wavelength } \\ \tau & \text { Position of window on velocity signal in wavelet transformation } \\ \Delta & \text { Diaphragm displacement } \\ \Delta \mathrm{T} & \text { Temperature difference } \\ \Delta \mathrm{P} & \text { Pressure difference } \\ \Delta \mathrm{t} & \text { Time resolution } \\ \Delta \mathrm{f} & \text { Frequency resolution } \\ \psi & \text { Stream function } \\ \psi & \text { Dilatation coefficient of wavelet transformation } \\ \Gamma & \text { Circulation }\end{array}$

\section{Abbreviations}

$\mathrm{A} / \mathrm{D}$

Analog to Digital

AR

Aspect Ratio

CCA

Constant Current Anemometry

CCD

Charged Coupled Device

$\mathrm{CCW}$

Counter Clock Wise

CTA

Constant Temperature Anemometry

CW

Clock wise 


$\begin{array}{ll}\text { ECG } & \text { Electrocardiography } \\ \text { FFT } & \text { Fast Fourier Transformation } \\ \text { LDV } & \text { Laser Doppler Velocimetry } \\ \text { LIF } & \text { Laser Induced Fluorescence } \\ \text { NS } & \text { Negative Slope } \\ \text { PMT } & \text { Photo Multiplier Tube } \\ \text { PS } & \text { Positive Slope } \\ \text { PSD } & \text { Power Spectral Density } \\ \text { SJ } & \text { Synthetic Jet } \\ \text { TFR } & \text { Time Frequency Resolution } \\ \text { TTL } & \text { Transistor - Transistor Logic } \\ \text { UAV } & \text { Underwater Autonomous Vehicle } \\ \text { UI } & \text { Uncertainty } \\ \text { VFD } & \text { Variable Frequency Drive } \\ \text { WVD } & \text { Winger Valley Distribution } \\ \text { ZNMF } & \text { Zero Net Mass Flux }\end{array}$




\section{Chapter 1}

\section{Introduction}

The formation, propagation, and interaction of vortex rings have been the subject of various researchers due to its wide application in nature and day-to-day life. In nature from simple starting jet to complex and large volcanic eruption, propulsion of aquatic creatures, flying of birds in the sky, discharge of blood from the left atrium to the left ventricular cavity in the human heart, formation of vortex ring is quite common. In industrial application such as controlling vertical and/or short take-off and landing of aircraft during transition flight, the roll-control of missiles, chimney flows, sewerage outfalls, manipulation of vortex ring for flow control such as blooming of jet, vortex guns in defense etc., vortex ring is identified as the prime flow feature. Vortices can be of variety of shapes and configurations depending on their axis of rotation and shear present in the flow and can be classified as line, sheet, tube, sphere and ring vortices. For example, line vortices form when fluid particles rotate around a line. A bundle of vortex lines forms a vortex tube generally seen in whirlpools or tornadoes and sheet vortices are formed when two streams of fluid having different velocities flow in same direction side by side as observed along the trailing edge of an aircraft wing. Similarly, the well-known mushroom shaped cloud produced by nuclear explosion with complicated vortex motion is an example of spherical vortex. Smoke ring produced by the smokers is an example of ring vortices where the axis of rotation forms a 
ring shape structure. Among all types of vortices discussed above, the ring vortices or vortex ring have an unusual property of self-convection widely known as self-induced velocity.

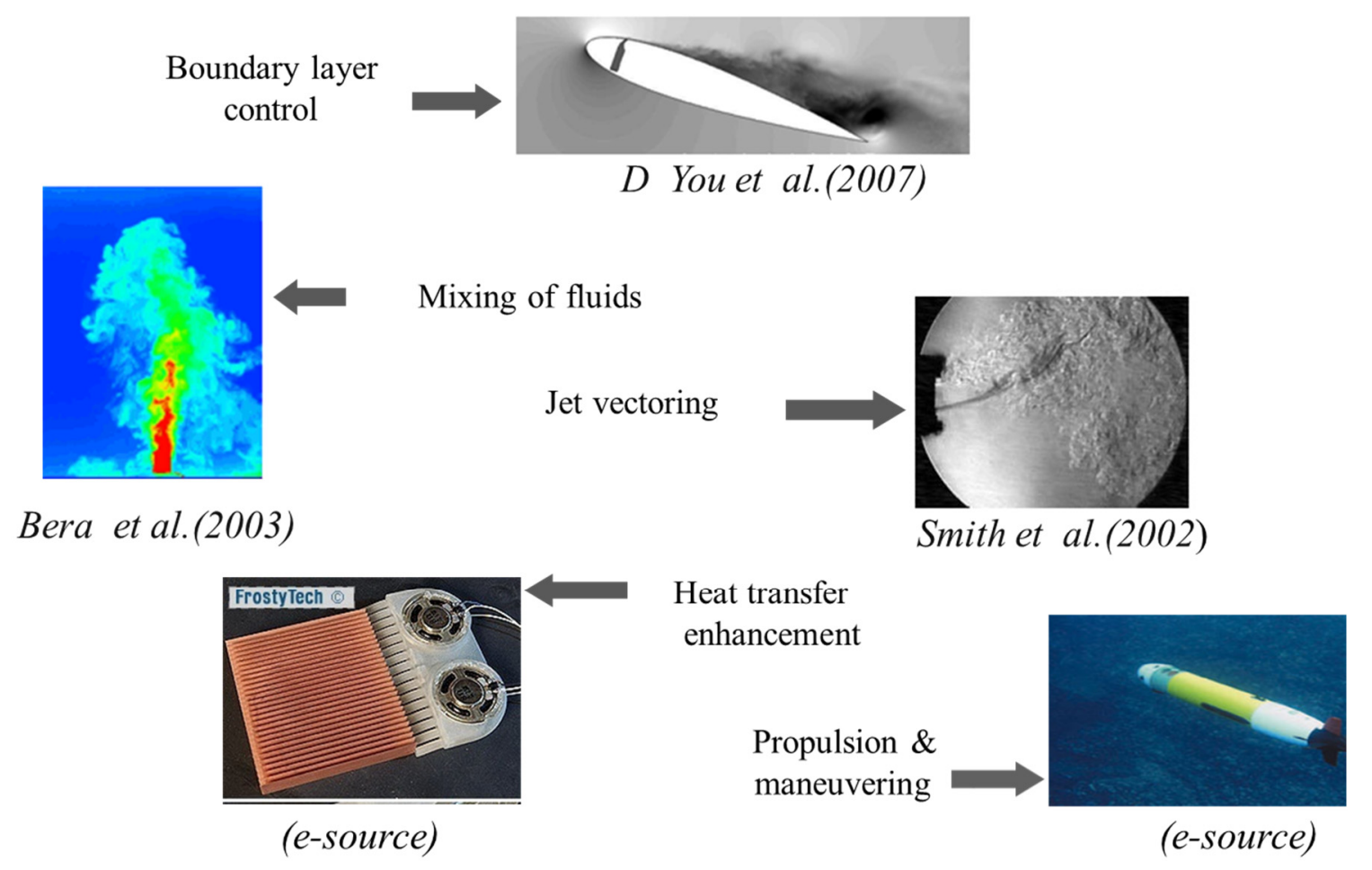

Figure 1.1: Various applications of synthetic jet in fluid flow control.

An isolated vortex ring moves forward due to its self-induced velocity, which can be described using Biot-Savart law. Marine creatures such as squid and jellyfish make use of vortex rings for their propulsion and maneuvering. They suck fluid from surroundings by expanding its body part and then squeeze their body to generate vortex ring. When vortex rings are generated through continuous suction and ejection of fluid from the environment in a cyclic manner, a train of vortex ring is formed thus transporting of momentum with the surrounding without adding any extra mass is possible. Consequently, system generating vortex ring experiences a thrust in opposite direction of vortex ring propagation. To exploit this advantage of generating thrust without any extra mass, many recent flow devices uses this technique to manipulate near wall flow dynamics. The vortex ring can be easily produced by piston-cylinder or oscillating-diaphragm mechanism in laboratory and it is 
possible to design for both macro to micro scale applications. As mentioned vortex ring can act as a vital tool for various applications like mixing, jet control, drag and lift control, boundary layer control, propulsion and maneuvering of underwater vehicle and many others.

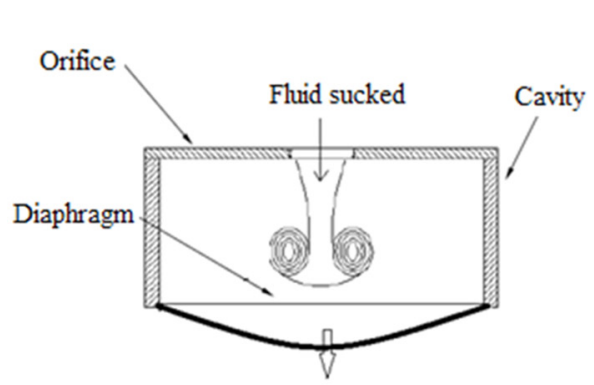

(a) Suction stroke

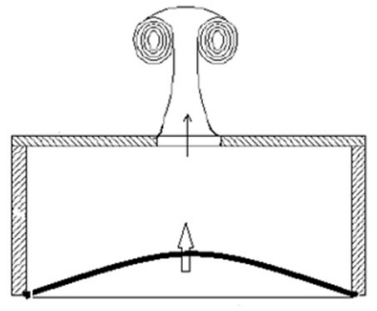

(b) Ejection stroke



(c) Suction stroke of next cycle

Figure 1.2: Formation of synthetic jet using diaphragm-orifice mechanism.

A simple cavity with a small orifice at one end and releasing acoustic waves at higher frequency and amplitude at the opposite end, researchers are able to generate a series of vortex rings. In the voyage of time, significant improvements and modifications to this device popularly known as synthetic jet, are undergone to suit various applications and has been discussed in a subsequent chapter. A synthetic jet actuation system typically consists of three elementary components namely, (a) a cavity having two open sides, (b) an oscillating boundary such as a diaphragm fitted with a mechanism that creates the oscillation or a crank-piston mechanism for controlling suction and ejection of fluid volume and (c) an opening at other end of the cavity through which fluid transport takes place with outside surrounding fluid. During backward stroke, cavity size increases, which allows ambient fluid to be sucked inside the cavity through the opening and is expelled outside during the forward stroke. During fluid motion in and out of the cavity shear layers formed at circumference of opening separate at the cavity edge and due to vorticity variation at outlet, shear layer rolls up to form a vortex ring. The vortex ring moves away from the 
orifice exit plane under the influence of self-induced velocity and thus synthesize a jet. The net mass transfer through the opening in one cycle is zero but there is a net transport of momentum to the surroundings. Therefore, the synthetic jet is often called as Zero net mass flux $(Z N M F)$ system.

\subsection{Motivation}

As an active device, synthetic jet with a train of large scale coherent structures and zero net mass flow rate have many practical and industrial applications in flow control. The simplicity of vortex generation, less moving parts, flexibility in size from micro- to macroscale and better adaptive control fulfills most of the requirements of modern flow control devices. Even though the study of synthetic jet as a flow control device, gained momentum since last 2-3 decades as numerous research works have been conducted on this topic. However, most of them considered air as a working fluid. The literatures on behavior of synthetic jet in underwater application are limited. However, the evolution of synthetic jet in shallow water and nature of rectangular synthetic jet where the bifurcation occurs is not available. Therefore, the characteristics of circular and non-circular synthetic jet quantitatively and qualitatively in water for quiescent and crossflow condition has been studied for various Reynolds numbers and Strouhal numbers.

\subsection{Objectives}

The convection, diffusion, and interaction among the coherent structures of a synthetic jet results in complex flow structures. The flow visualization gives an insight of flow structure whereas the velocity measurements such as instantaneous and time-averaged velocity field provide quantitative behavior. For qualitative study, the flow visualization is carried out with Laser Induced Fluorescence (LIF) and bulk-flow dye visualization technique using 
incandescent light. Hot-film anemometry (HFA) and five-beam Laser Doppler velocimetry (LDV) have been used for velocity measurements. The present study includes the characterization of synthetic jet in both quiescent flow and in crossflow condition. The broad objectives of the present thesis are the followings:

- Design and development of water tank fitted with synthetic jet generator on one of its sidewall for quiescent flow experiment and a torpedo shape model with built-in synthetic jet.

- Characterization of circular and rectangular shape synthetic jet in quiescent flow at different actuation frequencies and amplitudes using LIF and hot-film measurement.

- Both qualitative and quantitative characterizations of circular synthetic jet in torpedo shape model at various crossflow velocities and actuation frequencies.

The first objective has been achieved by designing and fabricating water tank with electromagnetic actuated diaphragm at one of its sidewall. The motor-cam synthetic jet to be put inside a torpedo shape model has been designed and fabricated for crossflow experiments.

The second objective is achieved by using both circular orifice of varying diameter and rectangular orifice of different aspect ratios at different actuation frequencies and amplitude of oscillations. The orifices are placed on synthetic jet generation unit mounted on one of the wall of water tank. The flow visualization is performed using LIF technique by capturing images on two orthogonal planes. The streamwise velocity component is measured using a S-type hot-film and analyzed them in time, frequency and combined timefrequency domain.

To achieve the third objective, synthetic jet with actuation system built-in inside the torpedo shape model is used. The model is positioned inside the test section of a water 
tunnel using dynamic model support system and then velocity measurement is carried out using LDV at different actuation frequencies and crossflow velocities. For qualitative study both the bulk-flow dye visualization and LIF techniques are employed.

\subsection{Organization of the thesis}

The thesis is organized as follow: Literature review relevant to present thesis has been reviewed in the Chapter 2. Experimental setup and measurement techniques are presented in Chapter 3. The flow field behavior of circular and rectangular synthetic jet in quiescent flow setup are reported in Chapter 4. Chapter 5 discusses the flow physics and characteristics of bifurcating and non-bifurcating synthetic jet of aspect ratio two in quiescent flow condition. The behavior of circular synthetic jet built-in in torpedo shape model in crossflow condition is reported in Chapter 6. The conclusion and scope of future work have been reported in Chapter 7. A brief overview related to the effect of controlling parameters and dimensionless numbers on the evolution of synthetic jet are reported in appendix-A. Appendix-B reports the information regarding the facilities used and uncertainty in experimental results. 


\section{Chapter 2}

\section{Literature Review}

The synthetic jet is a device widely used for applications where net momentum gain is important to control the flow or achieve thrust. The power supplied to the actuator is transferred to the fluid through oscillatory motion of a diaphragm resulting in momentum addition to the surrounding fluid. In this chapter, literatures on synthetic jet and its various applications are discussed in details.

The earliest work on synthetic jet has been found in Ingard and Labate (1950) where they showed generation of vortex rings using a simple cavity with a small orifice at one end and acoustic waves at higher frequency and amplitude at the opposite end. In the present study, experimental study of characteristic of synthetic jets at various conditions has been carried out. The literature on the various aspects of synthetic jet has been studied and summarized.

The synthetic jet finds itself in a wide variety of engineering applications; some of them are discussed in the following section. 


\subsection{Application of synthetic jet}

Flow control using synthetic jet has attracted a great deal of attention over the past decade due to its wide range of its potential applications. It can be used to improve mixing, heat transfer and combustion. Since the large-scale structures play a primary role in the transport phenomena, selective manipulation of these structures is desired in order to achieve an efficient control.

\subsubsection{Jet control}

Ho and Huerre (1984) suggested that that a flow can be modified by disturbing it temporally as the shear flow is extremely receptive to disturbances within a limited frequency band. On similar concept, Smith and Glezer (2002) controlled the deflection of primary jet by manipulating a small synthetic jet placed adjacent to the primary jet. They placed the coflowing synthetic jet near the exit of primary jet, the nozzles of both jets have the same length, but the aspect ratio for primary and synthetic jets are 6 and 25 respectively. In their experiment, they observed that entrainment of primary jet fluid by the adjacent synthetic jet lowers the static pressure near the flow boundary results in deflection of primary jet. Based on their observation, they concluded that synthetic jet is a better option for jet vectoring in comparison to the mechanical system.

\subsubsection{Spray technique and combustion control}

Another important application is in spray technique where synthetic jets are used to control the characteristics of sprays for various applications such as spray cooling and pesticide spraying. Pavlova et al. (2008) used synthetic jet for controlling the global behavior of spray such as droplet size, its distribution and concentration. They observed that the effect of flow control depends mainly on the relative strength of the synthetic jet with respect to 
that of the spray. Increase of air-fuel mixing at molecular level and entrainment of air for complete combustion of fuel has been studied by Ritchie et al. (2000) using nine synthetic jets along the circumference of the primary jet in their combustion experiments.

\subsubsection{Jet impingement cooling}

In recent years, there is an increasing demand of jet impingement cooling technique for electronic equipment and closely packed systems. Eibeck et al. (1993) used experimental as well as computational techniques to identify the secondary flow structures using synthetic jet pulsations impinging on a flat plate and found a remarkable increase in heat transfer. Zumbrunnen and Aziz (1993) also confirmed the heat transfer enhancement by pulsed impinging jets by a factor of two using water as a working fluid. Numerical study at various pulsation frequencies carried out by Freund and Moin (2000) suggested that a circular nozzle operating at Strouhal number of 0.2 provides better mixing due to larger spreading in downstream direction. Using synthetic jet impingement cooling over a heated plate, Pavlova and Amitay (2006) found that high frequency of pulsation of synthetic jet shows better performance as it can remove more heat than at lower frequency when the distance between the target area and synthetic jet is less. They described the reason to be breakdown of vortex ring into small structure at higher frequency before impingement causing an increase in heat transfer. They also reported that at same Reynolds number, heat removal by synthetic jet is three times higher than that of continuous jet due to presence of dominant coherent vortex structures in synthetic jet causing higher mixing with surroundings fluid through bulk motion. The impingement of synthetic jet having different shapes of orifice and aspect ratio over a heated plate is investigated experimentally by Chaudhari et al. (2010). Their observation showed that heat transfer using synthetic jet of rectangular shape orifice could be effectively used for cooling hot surfaces placed at lower 
axial distances from orifice exit while a square orifice is a much better option than rectangular orifice if there is no space constraint.

\subsubsection{Boundary layer control}

The ability of transferring net momentum to the main flow without adding mass makes synthetic jet suitable for boundary layer control in many applications. Modification of boundary layer over an airfoil using synthetic jet is reported by Amitay et al. (2001) where synthetic jet is used near the leading edge of an airfoil to control the flow reattachment points. A reduction of $45 \%$ in drag force and $100 \%$ increase in lift force has been achieved in their study. The interaction of synthetic jet with a flat boundary layer is investigated numerically by Mittal and Rampunggoon (2001). Their results show that synthetic jet is capable of forming a large recirculation zone only when the synthetic jet to cross-flow ratio is high. The experimental work of Amitay and Glazer (2002) using phase-lock velocity measurement, amplitude modulation and flow visualization on airfoil reports that onset of reattachment and separation are accompanied by the shedding of large-scale vortical structures. Hong et al. (2002) have used synthetic jet for control of boundary layer in adverse pressure gradient condition of crossflow. They observed that synthetic jet is more effective when the forcing frequency is in the range of those found in Tollmien-Schlichting waves. Using two-dimensional numerical simulations, Mittal and Rampunggon (2002) showed that synthetic jet in crossflow forms a recirculation zone giving a virtual aero shaping effect and consequently affects separation. An experiment investigation by Rathnasingham and Breuer (2003) presents a successful utilization of micro-synthetic jet array in controlling the near-wall turbulence of a turbulent boundary layer. They reported a maximum reduction of $30 \%$ in streamwise velocity fluctuation at the point of application. 
The effect is seen to persist up to several hundred and 50-150 viscous lengths in streamwise and spanwise directions respectively.

\subsubsection{Propulsion and maneuvering}

In all applications discussed above the major focus was on the flow field characteristic of synthetic jet outside the synthetic jet generator but in case of application in propulsion and maneuvering, the prime objective is the thrust experienced by the generator itself due to momentum transferred by the synthetic jet. In nature, many creatures such as jellyfish, squid, and shark use a train of vortex rings for their propulsion and maneuvering. The shapes and sizes of the vortex for these aquatic organisms vary depending on their body shape and method of vortex generation. For underwater research purpose, traditional underwater vehicles available in torpedo or box shape are propelled and docked using fan propeller. Fan propellers have their own advantages and limitations. It shows low energy consumption in torpedo shape body while docking is accurate and easier for box shaped design body. Optimized energy consumption and docking using fan propulsion system is difficult for streamline body because of poor controllability at low speed, less power to weight ratio and more moving parts. The synthetic jet propulsion and maneuvering systems meets maximum of the desired properties for underwater vehicle. Siekmann (1962) investigates the propulsion technique of sea creatures using simplified mathematical model of a pulsating jet issuing at the end of a tube with some assumptions. Works of Linden and Turner $(2001,2004)$ focus on the propulsion technique of squid, sea salp and jellyfish using vortex ring generated in the laboratory. Their investigations concerning the characteristics of a vortex ring such as circulation, impulse and kinetic energy revealed that for a formation number of 3.5, the vortex ring delivers maximum impulse for a given amount of kinetic energy and the ratio of vortex radii (core to ring) of 0.42 . Sometimes, the surrounding fluid 
has a crossflow/co-flow velocity depending on the orientation of the synthetic jet that affects the behavior of synthetic jet propulsion. Experiments conducted by Anderson and Grosenbaugh (2005) revealed that the ratio of co-flow to synthetic jet velocity is shown to more important than the formation number. Krieg et al. (2005) used synthetic jet for propulsion and maneuvering of underwater autonomous vehicle (UAV) and the relationship between thrust and formation number is established for synthetic jet. Both theoretical and experimental studies show a square dependency of thrust on actuation frequency and a maximum thrust for a given energy is achieved between formation number of 4 and 5.5. Similar observation has also been obtained by Thomas et al. (2005) using a voice coil driven synthetic jet, where they suggest that lower frequency between $2-5 \mathrm{~Hz}$ provides most efficient propulsion. In another study, Thomas et al. (2005) proposed an oval shape UAV that has been propelled and maneuvered by a number of synthetic jets. They

found that thrust increases proportionally with frequency. A successful application of synthetic jet for cruising, docking and transition is presented by Krieg and Mohseni (2008) where they examined the effect of actuation frequency and stroke ratio on the thrust produced by synthetic jet. They also observed adversely affected thrust at higher frequency is because of cavitation formed inside the cavity. Further improvement of the same work is reported by Krieg and Mohseni (2010) where they have developed a linear transfer-function model to approximate the thruster and vehicle dynamics.

\subsection{Continuous jet versus synthetic jet}

Since the sequence of vortex rings expelled out through the orifice or nozzle of a synthetic jet generation device synthesizes nearly similar mean velocity distribution and overall effect in downstream as unforced or continuous jet, it is called a synthetic jet. The studies by Smith and Swift $(2001,2003)$ compare synthetic jet, perturbed continuous jet $(5 \%$ of average velocity) and free continuous jet having rectangular orifice. The Schlieren images 
shown in Figure 2.1 indicate that the pulsation imposed on the continuous jet increases both the turbulence level in flow field and spreading in traverse direction. When the flow from orifice becomes fully cyclic and reversed as presented in Figure 2.1(c) for synthetic jet, flow field close to the jet exit becomes entirely different from that of continuous jet. The synthetic jet reveals less penetration but larger transverse spreading than the other two cases. The power spectra for the various cases show multiple harmonic peaks for both the synthetic and oscillating jet but single peak for continuous jet.



(a)

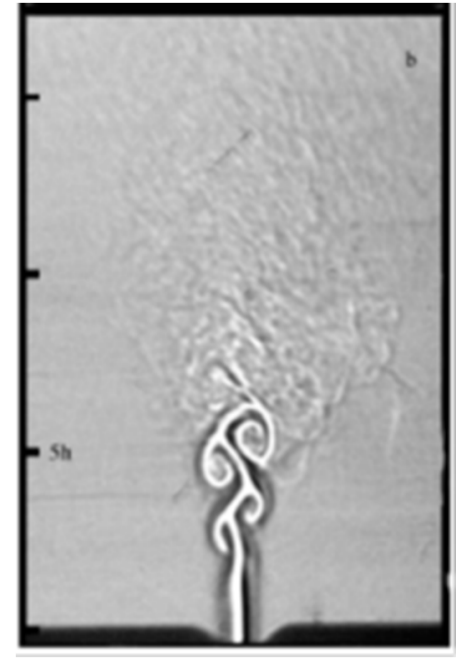

(b)

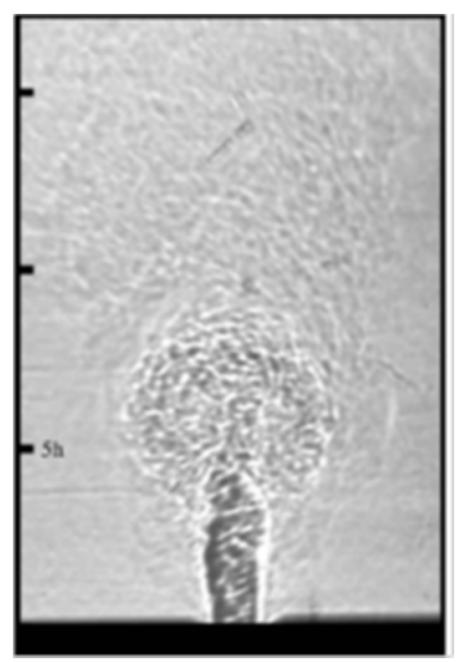

(c)

Figure 2.1: Schlieren images of (a) continuous jet (b) oscillating jet and (c) synthetic jet for $b / h=17$ and $\operatorname{Re}=2200$. (Smith \& Swift, 2001)

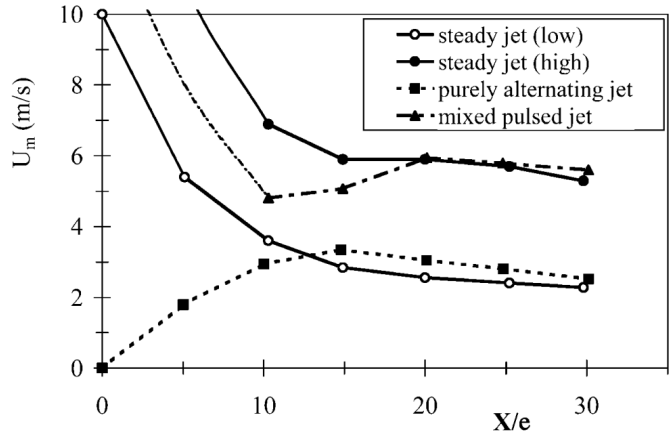

(a)



(b)

Figure 2.2: (a) Mean streamwise velocity profile along the jet centerline and (b) half width of jet ' $b$ ' as a function of distance from injection nozzle. Nozzle width (e) and breadth are equal to $1 \mathrm{~mm}$ and $100 \mathrm{~mm}$ respectively. (Bera et al., 2001) 
In contrast to the near field, the far field of both the synthetic and continuous jets under the similar conditions exhibit self-similar velocity field. Bera et al. (2001) conducted an experiment using a slit of dimension $1 \mathrm{~mm} \times 100 \mathrm{~mm}$ in four different kinds of jet flow. The characteristics of both the jets have been illustrated in Figure 2.2. The first one is a continuous jet at $10 \mathrm{~m} / \mathrm{s}$ (steady jet; low) which is same as the mean velocity of synthetic jet in downstream location (30 times of orifice width). Second one is a continuous jet at 20 $\mathrm{m} / \mathrm{s}$ (steady jet; high) and is having same mean velocity of synthetic jet at orifice exit. The third case is a mixed pulsed jet having the same mean velocity of synthetic jet at jet exit is chosen for comparison while the last one is synthetic jet. From velocity distribution shown in Figure 2.2 (a), the complete flow domain of synthetic jet is divided into two regions as near field and far field region. The near field region extends from jet exit to the point where vortex ring attains its maximum velocity and influenced by the suction period of next cycle, while the far field extends beyond the near field where vortex ring propagates, breaks up into tiny vortices and finally dissipates into the surrounding. Based on jet half width criteria, the spreading of all cases in traverse direction confirms the higher spreading of synthetic jet and the difference among the various cases increases in the downstream direction (Figure 2.2 (b)). From the non-dimensionalised cross-stream velocity distribution in the far field, they confirm the self-similar nature of velocity field of the synthetic and continuous jets.

\subsection{Synthetic jet formation criteria}

The mechanism of formation of vortex ring in synthetic jet is different from the formation mechanism of starting vortex ring of continuous jet. Similar differences are also observed in flow field where the vortex rings are generated with sufficient time lag. For such a flow field, the newly formed vortex ring are unaffected by the suction effect of the next cycle. On the other hand, the newly formed vortex ring in a synthetic jet is affected by the 
pulsating nature of the flow field developed especially close to the jet exit. The energy of vortex ring depends on many factors such as the diaphragm displacement and frequency of actuation. As stated earlier, if the newly formed vortex ring does not have enough energy to escape from the suction zone, partially or completely it is sucked back inside the cavity. The self-induced velocity of vortex ring near the exit depends on various factors as the energy of shear layer supplied to the forming vortex ring, curvature of orifice, duration of forward and backward stroke, orientation of orifice and diaphragm and environmental conditions. James and Jacobs (1996) investigated the characteristics of a synthetic jet in water using a flush mounted oscillating diaphragm and concluded that synthetic jet is formed when the excitation level of the actuator exceeds a given threshold. A major development in the theory of synthetic jet formation has been put forward by many researchers. Gharib et al. (1998) provides the concept of a non-dimensional number widely known as "formation number". It is defined as the ratio of slug length (L) to the hydraulic diameter of orifice (D). In other word, the formation number is the limiting value for a given geometry after that any extra circulation generated by ejecting shear layer ceases to flow inside the core region of the vortex ring. Once the formation number is reached, this extra ejecting fluid gets pinch-off from the leading vortex ring forming trailing jets. This subsequent generation of trailing vortices leads to the formation of series of vortices similar to the Kelvin Helmholtz instability. 


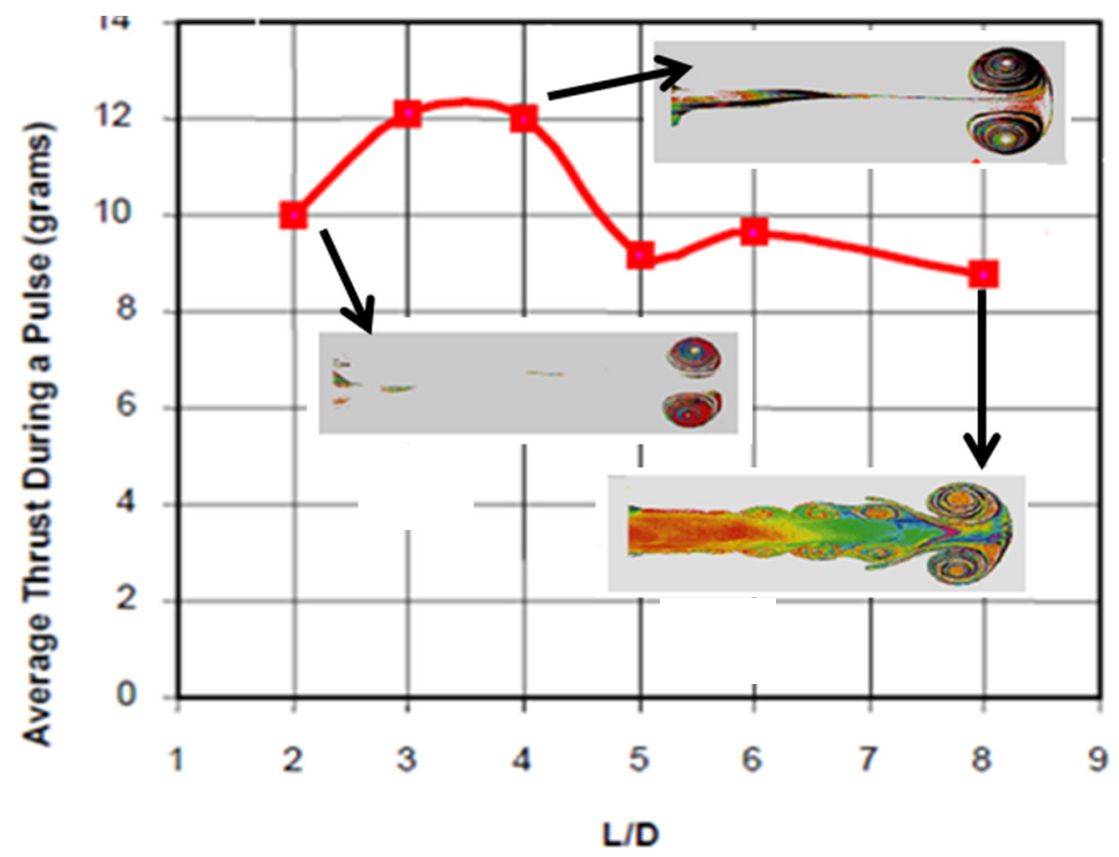

Figure 2.3: Images of vortex ring at three different $L / D$ ratio $(2,4$, and 6$)$ and distribution of average thrust with respect to $\mathrm{L} / \mathrm{D}$ ratio (Gharib et al., 1998; Kruger, 2003).

In Figure 2.3, three different types of vortex formation are shown for a circular orifice with piston-cylinder actuation system. When the formation number (L/D) is less than a value of 4 , the vortex ring is formed but the maximum value of circulation is not achieved. At a formation number equal to 4 , the maximum size of vortex ring occurs and above this value the formation of trailing jet begins and for large formation number leading vortex ring is followed by a strong trailing jet. Rosenfeld et al. (1998) through numerical simulation leads to a conclusion that formation number strongly depends on the velocity profile of discharge flow and weakly depends on temporal velocity variation of the oscillating boundary, but total circulation depends on both the discharge velocity profile and velocity variation. Similar observation was also observed by Glezer (1998) where he showed that formation number based on simple cylindrical slug model does not account for geometrical condition of vortex generator believed to the velocity of the diaphragm and velocity variation of discharge fluid. For more accurate prediction for initial circulation of vortex ring, an additional non-dimensional parameter $\Gamma / v$ is needed, where $\Gamma$ is the circulation of vortex ring and $v$ is the kinematic viscosity of fluid. Mohseni and Gharib 
(1998) predicted the non-dimensional circulation calculated from the translation velocity of vortex ring to be in the range of 1.77 to 2.07 for successful formation of vortex ring using analytical model. Since the vortex ring size increases during its spatial evolution, the feeding shear layer is pushed towards the axis of symmetry and the detachment of the ring occurs when its velocity becomes more than the local axial velocity of feeding shear layer. Shusser and Gharib (2000) analytically try to figure out the situation where the pinch-off starts based on energy and circulation of vortex ring. Mohseni et al. (2001) suggested two methods to control the pinch-off process to increase the vortex size and its strength. In both the methods, the delay in vortex detachment is achieved either by (i) varying the velocity of the piston or (ii) by changing the orifice radius linearly during expulsion period. An increase of around $25 \%$ in the normalized circulation and $40 \%$ reduction in the normalized energy are obtained using the above two methods. Contrary to the concept of Mohseni and Gharib (1998), Liden and Turner (2001) concluded that critical value of formation number calculated based on slug method or pinch-off time is a function of volume constrain related to vortex core volume, ejected volume and added mass from ambient. Using the order of magnitude analysis, a jet formation criteria has been proposed by Utturkar et al. (2003) in which they showed that for successful formation of vortex ring, the ratio of Reynolds number divided by the square of Stokes number must be greater than some constant that varies with orifice geometry. An over pressure correction in the slug model is suggested by Kruger and Gharib (2003), reveals that over-pressure is associated with the acceleration of ambient fluid caused by the leading vortex ring in form of added and entrained mass. Their observation indicates that the over-pressure adds an additional impulse during the vortex ring formation without affecting the trailing jet. The parallel flow assumption in slug model is extensively studied by Krueger (2005). He found that during flow initiation at nozzle exit, over-pressure accelerate the ejecting jet fluid radially outwards and generates curved 
streamline. This in turn contributes to the circulation, which is not accounted in the parallel flow assumption of slug model. Lower L/D ratio results in higher gradient in radial component of velocity and thus error in calculation of total circulation is large and cannot be neglected.

\subsection{Parameters affecting the synthetic jet formation}

The jet formation is found to vary with various parameters some of which are discussed below.

\subsubsection{Synthetic jet generators}

The oscillating boundary of cavity is an important part of a synthetic jet generation device. The oscillation of one of the boundaries causes a pressure difference across the cavity and the surrounding fluid leading to ingress and egress of fluid into and out of the cavities respectively during operation. Some of the common methods of generating oscillating boundaries are motor-driven piston-diaphragm, acoustic loudspeaker-diaphragm, and piezoelectric-driven diaphragms and stacked recurve benders.

\subsubsection{Jet exit condition}

The synthetic jet exit condition can be broadly classified into two groups: first where the length on which shear layer is formed continuously changes from maximum to minimum value while in other case it remains constant throughout the cycle. The example of first case is piston-cylinder with cylinder opening acting as orifice, where the piston moves forward and the cylinder surface responsible for shear layer formation continuously decreases in height. The example of the other group is an orifice or nozzle where the contact surface over which shear layer forms remains constant. Cater et al. (2004) through experimental investigation concluded that the vortex ring produced by piston gives 
maximum circulation with axisymmetric distribution of vortex core than the diaphragmorifice as shown in Figure 2.4.

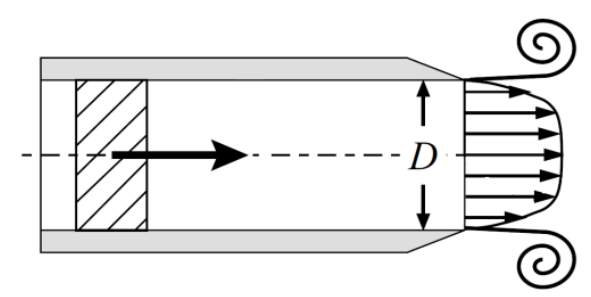

(a)

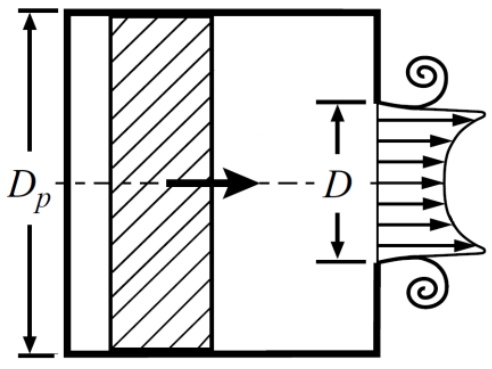

(b)

Figure 2.4: Exit velocity distribution of (a) piston-cylinder mechanism and (b) diaphragm-orifice mechanism (Kruger, 2005).

\subsubsection{Cavity design and diaphragm location}

The design of cavity has been found to affect the characteristics of the synthetic jet. Utturkar et al. (2002) have done two-dimensional numerical simulation on five different types of cavity designs with various diaphragms positions keeping the orifice exit area and fluid expelled from the cavity constant. The simulation is performed in quiescent flow as well as in crossflow condition. No significant changes in the velocity profile, circulation and energy distribution among the various cases have been noticed though one case shows a vortex dipole to undergo a substantial drift, Gallas et al. (2004) studied numerically three different orifice thickness to diameter ratios for a constant Reynolds number and concluded that the formation of synthetic jet is possible for a ratio of 0.68 , while for other two cases, the part of the synthetic jet is observed to be ingested back into the cavity.

\subsubsection{Velocity program of actuator}

The relationship between circulation and velocity variation at orifice exit depends on spatial and temporal distribution of velocity. On the other hand, the exit velocity depends on temporal velocity variation of actuator. Numerical investigation carried out by Rosenfeld 
et al. (1998) at constant Reynolds number, revealed that the maximum circulation in vortex ring occurs for impulsive velocity program of actuator as compared to the non-impulsive velocity program. Kruger and Gharib (2003) conducted experiments on two velocity programs, one with jet exit velocity decreasing for majority of ejection period while the other having jet exit velocity increasing for the majority of ejection period. They used varying $\mathrm{L} / \mathrm{D}$ ratio between 2 to 8 and observed that the propulsive quantity of starting jets is less for decreasing exit velocity program than that of increasing velocity program. The investigation for different ratios of ejection to suction period and actuation frequencies has been documented by Thomas et al. (2005) and an optimum value of the ratio of ejection to suction period is obtained.

\subsubsection{Orifice shape}

As discussed earlier, it is expected that the orifice shape have profound effect on the synthetic jet characteristics similar to continuous jet. The shape of orifice can be broadly classified into two types, circular and non-circular. Both the shapes have their own field of applications based on their advantages and disadvantages. The presence of sharp corners in non-circular orifice has unequal distribution of convection or self-induced velocity. According to Bio-Savart law, the segment of vortex rings nearer to the corners has larger curvature and thus moves faster because of higher convection velocity compared to the other portion of the ring. Because of this velocity difference, vortex ring is stretched causing the certain portion of the ring to move towards the center of vortex core while remaining portion of vortex ring moves away from the center. This differential movement of different parts of the ring gives rise to a phenomenon called axis switching which leads to faster breakdown of vortex rings. Using numerical simulations of elliptical shape orifice, Kiya et al. (1992) presented the sequence of vortex ring deformation in downstream 
direction at different time instances in all three orthogonal planes and is shown in Figure 2.5. The sequence of images in Figure 2.5 (a) demonstrate an axial switching phenomenon primarily responsible for large entrainment of surroundings fluid into a synthetic jet. The higher entrainment and turbulence of non-circular jets make it more unstable than the circular ones because of which non-circular rings are preferred for applications where mixing and heat transfer is of prime importance. The characteristics of synthetic jet of a rectangular as well as dual-circular orifice have been studied by Watson et al. (2003) where they showed that the circulation of the dual-circular orifice provides higher circulation in comparison to single orifice of equivalent area.

From thrust and propulsion point of view, the circular rings are found to be more efficient than the non-circular rings because circular rings provide higher circulation energy up to a longer distance. Using rectangular shape orifice, Auerbach and Grimm (1994) could show vortex splitting into 2, 3 and 4 secondary vortices. Hussain and Husain (1989) and Grinstein (2001) also reported the vortex ring bifurcation for an elliptical jet having an aspect ratio of 4. Watson et al. (2003) through experimental observation concluded that fluid leaving a rectangular orifice of a given exit area is more likely to be turbulent than the circular orifice of same exit area even though the peak exit velocities of the both the orifices are the same. The effect of different orifices (circular and regular polygons having 3 to 6 sides) on synthetic jet impingement cooling over a heated plate at different span-wiselocation perpendicular to jet exit direction is studied by Kanamori et al. (2011). 


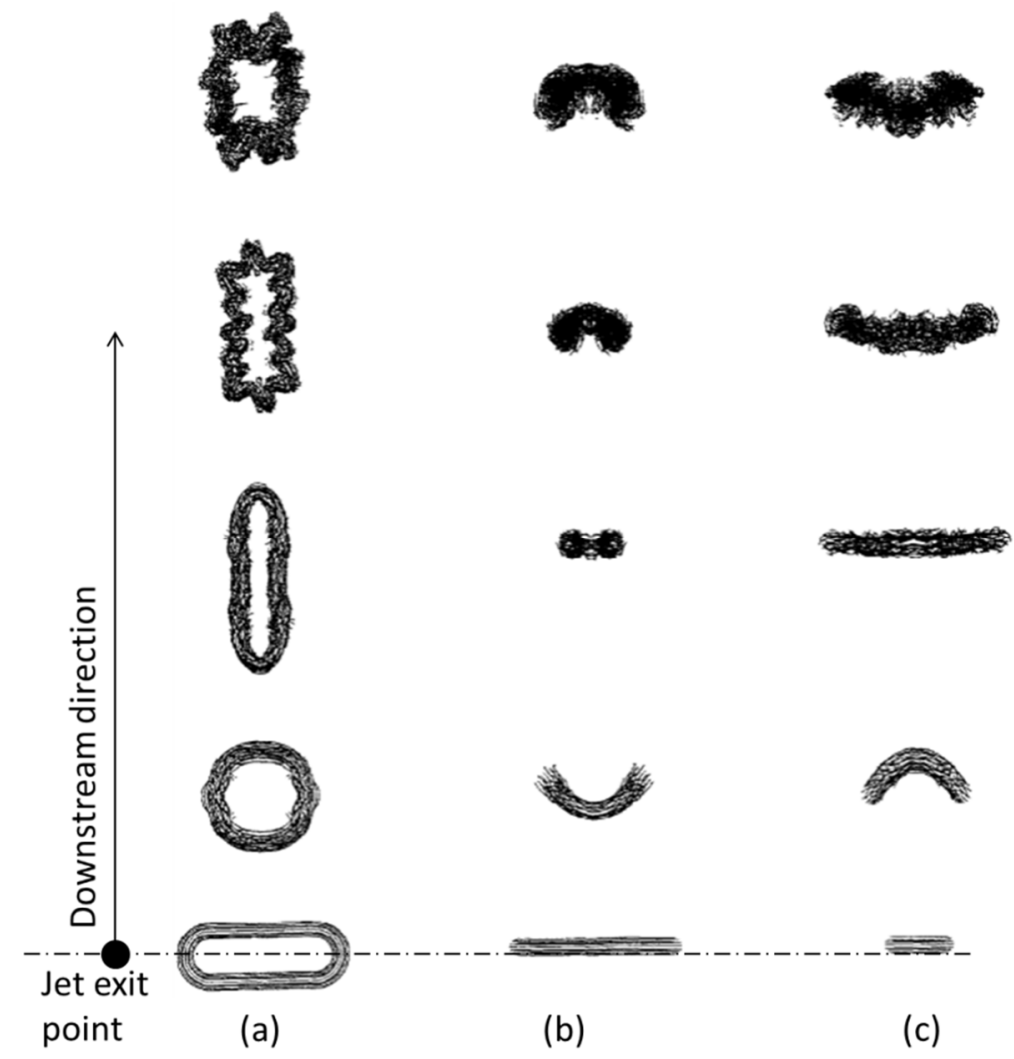

Figure 2.5: Simulated pattern of axial switching (a) in planes perpendicular to synthetic jet centerline (YZ), (b) in major axis plane of orifice $(\mathrm{XY})$ and (c) in minor axis plane (XZ) at different stages of vortex evolution (Kiya et al., 1992).

To achieve heat transfer enhancement using synthetic jet, Chaudhari et al. (2010) used circular, square and rectangular shape orifices of same hydraulic diameter with different aspect ratios of rectangular orifice. They observed that at lower axial distance the average Nusselt number is higher for rectangular shape orifices having aspect ratio in range of 4 to 6. At larger axial distances, the performance of square orifice is better than the other cases considered. They also found that circular and rectangular shape orifice nearly gives same amount of cooling at far away from jet exit. Based on their results, they concluded that a rectangular shape orifice with larger hydraulic diameter but having a smaller aspect ratio would be a better option for synthetic jet cooling. 


\subsubsection{Actuation frequency and amplitude of diaphragm displacement}

The effect of frequency and amplitude of diaphragm displacement are proven to be two important non-geometrical parameters as slight change in these parameters has a dominant effect on the characteristics of synthetic jet. The formation number discussed in previous section is a function of frequency and amplitude of diaphragm displacement. The corresponding non-dimensional numbers associated with these quantities are Strouhal number and Reynolds number. Individual effect of frequency and diaphragm displacement can also studied by varying them independently.

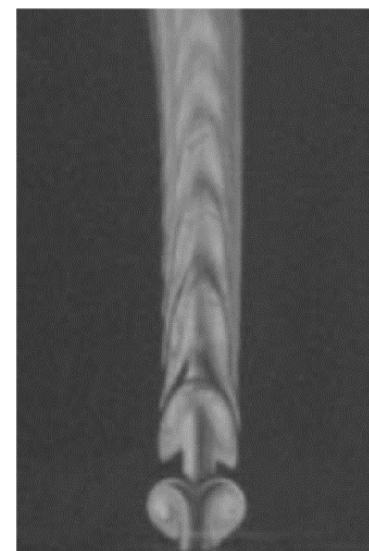

(a)



(b)



(c)

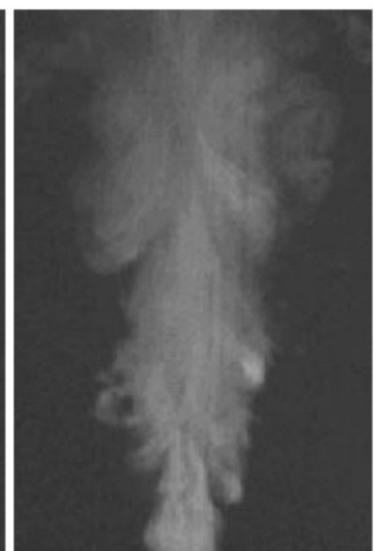

(d)

Figure 2.6: Flow field images of (a) Laminar jet at $\mathrm{Re}=3336, \mathrm{St}=\mathbf{0 . 3}$, (b) Laminar rings at $\mathrm{Re}$ $=7784, \mathrm{St}=0.012$, (c) Transitional jet at $\mathrm{Re}=11121, \mathrm{St}=0.009$ and (d) Turbulent jet at $\mathrm{Re}=$ 66991, $\mathrm{St}=$ 0.009. (Cater and Soria, 2002)

Cater and Soria (2002) generated four different types of synthetic jet flow pattern (Figure 2.6 ) using different Reynolds numbers and Strouhal numbers calculated based on overall (time and space) averaged jet exit velocity. A few studies have been carried out to investigate the effect of L/D ratio, Reynolds number and Strouhal number. However, most of the studies investigated the effect of the above parameters of a circular orifice in a quiescent flow condition taking water as working fluid. Some of them are discussed here. Didden (1979) found the vortex formation criteria based on circulation energy associated for $50 \mathrm{~mm}$ circular orifice having $\mathrm{L} / \mathrm{D}=1.4$. James and Jacobs (1996) studied the synthetic 
jet characteristics at higher actuation frequency at which the jet is found to be turbulent. Mallinson et al. (2004) have carried out both computations and experiments using hot-film anemometer to quantify the effect of frequency on turbulent synthetic jet. A study of synthetic jet has been carried out (Shuster and Smith, 2007) at various Reynolds numbers and $\mathrm{L} / \mathrm{D}$ ratio where they compared their results with the corresponding continuous jet. They showed that the synthetic jet provides better spreading but the decay of centerline velocity is more for synthetic jet in comparison to continuous jet. Cicca and Iuso (2007) investigated a detailed near field behavior of synthetic jet at various Reynolds numbers (1290-3400) based on average jet exit velocity and actuation frequency (0-5 Hz). Gao et al. (2008) studied experimentally the effect of Reynolds number on the characteristics of leading vortex. They observed the pinch-off of the leading vortex when Reynolds number exceeds 3000 and presented the circulation and energy of the leading vortex at various Reynolds number. A criterion for jet formation has been proposed by Utturkar et al. (2006), where it has been shown that jet formation is governed by frequency (Strouhal number) or amplitude of oscillation of the actuator (Reynolds number and Stokes number). A prediction of theoretical model of circular synthetic jet created in an incompressible flow has been developed by Tang and Zhong (2005). In their study, the relation between the performance and operating parameters such as Reynolds number, stroke length are established. Smith and Glazer (1998) studied a rectangular synthetic jet in air where the effect of Reynolds number on the characteristics of flow evolution and far field has been analyzed. Gilarranz et al. (2005) using hotwire studied the characteristics of synthetic jet in terms of velocity of the synthetic jet at various frequencies and orifice aspect ratios. Raju et al. (2005) studies the effect pressure loss due to sudden expansion and contraction at the rectangular orifice exit for Stokes number varying between 10 to 25 and a range of Strouhal number (0.6-2.39). Cannelle and Amitay (2007) investigated the transitory behavior of 
synthetic jet using hotwire and PIV techniques. For their study to transitory evolution, frequency is varied in the range $300-917 \mathrm{~Hz}$ while Reynolds number is set to a value between 85 and 365 .

\subsubsection{Compressibility effect}

The effect of compressibility of synthetic jet fluid is more obvious for air than water. In the study of synthetic jet produced by piston-cylinder mechanism, Crittenden and Glezer (2006) observed that compressibility has significant effect on the shape of the time-periodic pressure variation. They showed that pressure distribution is not similar during the blowing and suction cycles. A phase shift between the peak piston displacement and the corresponding pressure maxima has been noticed. Contrary to the general belief, Sharma (2007) through numerical simulation taking air as working medium concluded that behavior of actuator cavity is compressible at all frequencies beyond Helmholtz resonance frequency in the cavity.

\subsubsection{Orifice inclination}

The inclination angle of orifice is expected to alter the flow field of synthetic jet. The inclination can be of different types depending on the angle made by the axis of the symmetric shear layer with free surface or the wall on which the nozzle or orifice is mounted. Webster and Longmire (1998) showed that the primary or leading vortex ring has non-uniform distribution of circulation around its circumference. A complex structure, which include primary vortex ring and branched vortex tubes has been seen to exist. In addition, their results reveal that vortex rings migrate away from centerline with increasing inclination. 


\subsection{Synthetic jet behavior in non-quiescent flow condition}

In non-quiescent flow conditions such as crossflow, counter-flow and co-flow conditions, the characteristics of synthetic jet such as formation and propagation of vortex ring in downstream direction, entrainment with surroundings fluid, spreading in transverse direction, decay rate and others properties are expected to be different from those in quiescent condition. The jet control, boundary layer manipulation, propulsion and maneuvering are the applications where synthetic jet encounters non-quiescent flow condition. The control of adverse pressure gradient in boundary layer has drawn attention of many researchers in the past. Many active and passive techniques have been implemented but the problems associated with efficiency of device, space restriction and adaptive control for modifying boundary layer using minimum moving parts and making the overall system lightweight. The passive devices modifies the flow through the changes in geometry without any expenditure of energy. On other hand, the active control uses energy to modify the flow. Various active flow control methods are discussed in detail by Jahanmiri (2010) in his report on flow control. The active methods such as constant blowing or suction, flapping or vortex generators are either insufficient or impractical for boundary layer control due to various reasons. A process involving the suction of low momentum fluid from the boundary layer and ejection of the same fluid at higher momentum in the boundary layer periodically is one of the efficient methods of flow control in recent years, and is known as synthetic jet. Fundamentally, synthetic jet is a vortex generator generally used to control the flow especially in the boundary layer with adverse pressure gradient by transferring momentum and consequently increases shear stress. In comparison to steady blowing or suction, synthetic jet needs 1-2 order less magnitude of momentum to achieve equivalent effect. If the parameters of synthetic jet are such that the jet vortices are kept within the boundary layer, a similar effect as produced by passive vortex generator can be 
obtained (Qayoum et al., 2009). The behavior of circular synthetic jet in crossflow has been studied by Lee et al. (2003) for a frequency range of $0-3600 \mathrm{~Hz}$ in wind tunnel to control near wall flow field. A piezoelectric actuator has been used to actuate the boundary layer at the instability frequency. They concluded that the actuator should be operated at frequency to produce strong vortices to control the boundary layer. Zhou (2010) and Chaudhry and Zhong (2014) and Gorden et al. (2004) investigated the performance of synthetic jet in crossflow and compares with continuous jet. They found that at constant velocity ratio, the synthetic jet penetration decreases with increasing Reynolds number and Strouhal number. The spreading of synthetic jet is also found to be more than that of continuous jet in the near field. Zhong (2014) carried out experiments for continuous and synthetic jet in crossflow and reported the self-similar time-averaged field of both types of jet in far downstream.

\subsubsection{Synthetic jet in parallel-flow condition}

In co-flow condition, the resultant velocity of vortex rings is the summation of induced velocity of the synthetic jet and flowing fluid velocity while in counter-flow conditions the induced velocity is opposite to that of flowing fluid. A study made by Krueger (2003) showed that in the presence of co-flow, the formation number (based on slug model) decreases with increasing velocity ratio of co-flow and synthetic jet. They found the formation number is to be 4 without any co-flow but formation number drops to 1 or below when the velocity ratio of co-flow increases. From their experiments on synthetic jet produced using a pipe in a co-flow condition, Anderson and Grosenbaugh (2005) concluded that the velocity ratio is more important parameter than the $\mathrm{L} / \mathrm{D}$ ratio in co-flow conditions. They also observed that the isolated single vortex ring without trailing jet is formed when the synthetic jet velocity is two times higher than the co-flow velocity and L/D $=4$. 


\subsubsection{Synthetic jet in crossflow condition}

In parallel flow or quiescent flow, the vortex ring, the shape of which depends only on the shape of jet exit, is found to be simple but additional vortices are formed in crossflow. The stretching and tilting of vortex ring further increases the complexity of the flow field in crossflow condition. In experimental study of round continuous jet in crossflow by Kelso et al. (1996) and square continuous jet by Sau et al. (2004) have illustrated the formation of horseshoe vortex, wall vortex and upright vortex along with counter rotating vortex pair (part of vortex rings) are shown in Figure 2.7. These vortices particularly horseshoe, wall and upright vortices are expected to be present in synthetic jet ejecting into a crossflow. The most interesting feature of jet (continuous or synthetic) in crossflow is the formation of counter rotating vortex pairs. Jabbal and Zhong (2008) observed three distinct types of vortical structures for synthetic jet in crossflow namely hairpin vortices, stretched vortex rings and tilted/distorted vortex rings when jet interacts with zero pressure gradient laminar boundary layer. For velocity ratio between 0.1 and 0.32 , the hairpin vortices with and without secondary vortices are formed: in the intermediate range of velocity ratio, the stretched vortex rings appear while distorted vortex rings are seen at higher velocity ratio.

The flow physics associated with synthetic jet in crossflow involves the formation of a train of discrete vortical structures, which locally promotes significant levels of mixing between the outer flow and flow near the wall helping the boundary layer to become reenergized (Prince et al., 2012). The interaction of synthetic jet with wall boundary layer of a crossflow has been studied by Zhong et al. (2005) at low Reynolds number using a cavitydiaphragm device for generating jet. One cycle of synthetic jet forms two vortex rings, one during backward stroke where the vortex forms inside the cavity while the second one is generated outside the cavity during the forward stroke. The effect of Reynolds number, velocity ratio and Strouhal number on vortical structures of synthetic jet is demonstrated. 
They found that the formation of vortex inside the cavity might contribute to a chaotic and turbulent fluid to be ejected in the subsequent cycle.

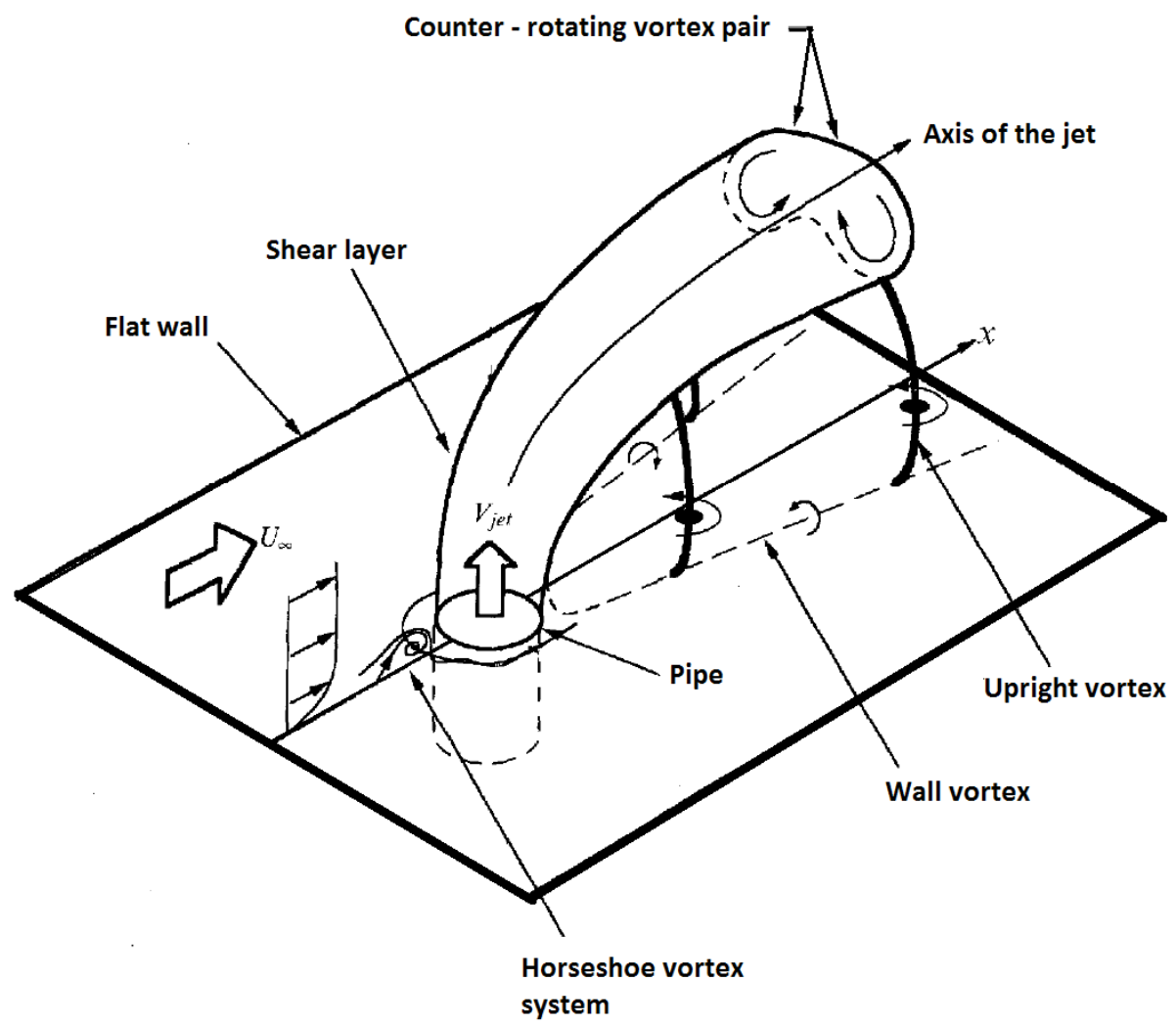

Figure 2.7: Schematic of different types of vortices resulting from jet in crossflow. (Kelso et al., 1996).

The experimental observation of Gorden and Soria (2002) shows that $25-40 \%$ and $95 \%$ of volume of fluid is drawn into the orifice cavity from fluid outside the cavity within region that is 0.2 and 3 diameters from the wall respectively. Liddle and Wood (2005) investigated the interaction of two synthetic jets in crossflow and suggested that input phase difference between the two jets have a strong influence on the stability of synthetic jet. The numerical simulations for two cases namely synthetic jet perpendicular to crossflow and inclined synthetic jet to crossflow have been carried out by Shuster et al. (2005). Duvigneau and Visonneau (2006) used an automatic optimization procedure coupled with flow solver for enhancing the lift force of an aerofoil. In their study, the optimized parameters are 
momentum coefficient, frequency and angle with respect to the wall. Wang et al. (2010) compares the jet velocity, flow flux, momentum flux and jet width at different duty cycles (suction period/blowing period) of two-dimensional synthetic jet. They found that the distributions of mean and turbulent quantities have similar features regardless of duty cycles. However, vortex pair generated at higher value of duty cycle convecting faster exhibits stronger entrainment and higher momentum. Sahni et al., 2010 observed in their study that synthetic jet in crossflow yields three distinct types of effects on mean flow: (a) reduced velocity just upstream of the jet, (b) higher velocity downstream of the jet and (c) thickening of the boundary layer downstream of the jet.

\subsubsection{Evolution of vortex ring in crossflow}

In quiescent flow, the spacing between the vortex rings of synthetic jet depends on the selfinduced velocity of vortex ring and the rate of vortex generation i.e. actuation frequency. In the presence of external crossflow, the vortex rings convect in the direction of crossflow after emerging from the orifice. Because of the nonlinear interaction between the crossflow and vortex rings, the spacing between the vortex rings expected to be strongly affected by the crossflow. The suction and expulsion stroke of oscillating diaphragm during one complete cycle of synthetic jet affects the boundary layer formed around the orifice. This is because of the variation for fluid ingested/ejected around the circumference during ingestion/ejection (Lardeau and Leschziner, 2011). The vortices at upstream side directly meet the cross-stream flow where it opposes the vortex roll up and thus weakening its strength. As the angle between the cross-stream and the local axis of vortex ring changes along the circumference of orifice from upstream side to downstream side, the opposing nature of cross-stream decreases beyond a location promoting the vortex roll up. Therefore, the vortices in the downstream side are stronger and survive longer than the vortices in upstream side, and these downstream side vortices strongly affect the near wall flow (Kim 
et al., 2010). Zhong et al., (2005) reported that due to varying scale of interaction, the vortices in upstream/downstream side become intensified/weakened by the resident vorticity in the boundary layer. The formation of vortex ring in crossflow is more sensitive to the velocity ratio as compared to any other parameters in such flow configuration. Jabbal and Zhong (2010) concluded from their PIV measurements on circular synthetic jet issuing to a flat plate boundary layer that at low velocity ratio $(\mathrm{VR}=0.32)$, the upstream side of vortex ring is found to be weaker than the downstream side leading to asymmetric development of vortex ring. Similar observation are also reported by Chaudhry and Zhon (2012), where the frequency is varied between 1 and $6 \mathrm{~Hz}$ with an increment of $1 \mathrm{~Hz}$ while the velocity ratio is altered between 0.1 and 0.5 for each actuation frequency.

Milanovic and Zaman (2005) reported that the jet penetration height and its trajectory is solely a function of momentum flux ratio, which is equivalent to the velocity ratio. Various researchers have reported the formation of hairpin or stretched vortices at lower velocity ratio whereas tilted and distorted vortex rings at higher velocity ratio. The hairpin vortices remain within the boundary layer whereas the stretched vortex ring eventually penetrates the boundary layer in far downstream. The reasons lie in the higher actuation frequency and/or vorticity content that control the interaction of consecutive vortex rings and results in increased penetration depth as compared to the hairpin vortices (Jabbal and Zhong, 2010). Crook et al. (2002) using a circular synthetic jet in crossflow in water actuating between 2 and $10 \mathrm{~Hz}$ concluded that the velocity ratio and vortex separation distance are key factors influencing the synthetic jet in crossflow. Zhou and Zhong (2010) numerically analyzed the transition between the hairpin vortices and tilted vortex rings and observed that at velocity ratio equal to 0.35 , the transition to turbulent flow is nearly unaffected by non-dimensional actuation frequency (Strouhal number) when lies in the range of $0.2-0.8$. For the velocity ratio between 0.4 and 0.8 , the transition from 
laminar to turbulent flow is strongly affected by Strouhal number in range of $0.8-2$. At a particular frequency, depending on the velocity ratio, hairpin vortices, stretched vortex rings and tilted-distorted vortex rings are observed. However, the study of behavior of synthetic jet is not available in the literature when it is mounted on a curved surface or operating at higher velocity ratio.

\subsection{Closure}

This chapter reports the literature related to characteristics and flow control applications of the synthetic jet. A short review comparing the properties of continuous jet with synthetic jet is also covered. Literature related to various geometrical and operating parameters, which control the behavior of synthetic jet has been discussed. The criteria for synthetic jet evolution in quiescent, co-flow and crossflow conditions are also discussed. The literatures on the behavior of synthetic jet in underwater applications are limited therefore creates the subject matter of the present research is very important for design exercise related to underwater applications. 


\section{Chapter 3}

\section{Experimental set-up and measurement techniques}

In Chapter-1, the motivation behind the present thesis work has been outlined and consequently the objectives are specified. In order to achieve those objectives, various measurement techniques and experimental set-ups used are presented in this chapter. Since the problem addressed in this thesis are quite complex in nature due to dependency of synthetic jet on various operational and geometrical parameters, a series of experiments are performed in order to understand the flow structure at various flow conditions. Initially, the characteristics of synthetic jet in quiescent flow condition are investigated using synthetic jet generator mounted on one of the sidewall of a water tank. The knowledge and experience gained out of these experimental findings are utilized in designing and fabricating a torpedo shape model with a built-in synthetic jet. The torpedo shape model with built-in synthetic jet facilitates to understand the behavior of synthetic jet in quiescent as well as in crossflow condition particularly for underwater vehicles. It is expected that the flow characteristics for a torpedo shape model is different from that of tank-wall mounted synthetic jet because of obvious change in the flow configuration. For qualitative measurements, the bulk-flow dye visualization and laser induced fluorescence techniques are used, whereas the velocity 
field is measured using hot-film anemometry and Laser Doppler Velocimetry (LDV) for quantitative assessment.

Brief description of the common peripheral devices used in the experiments such as hot-film calibration set-up, laser and its accessories, three-dimensional traverse, high-speed video camera and water tunnel are provided in appendix-B. The uncertainty associated with measurement techniques as well as measured values are discussed in appendix-B. The design details of the different experimental set-ups and methodology are presented in the following sections.

\subsection{Experimental set-up}

To achieve the objectives of the present work, two different experimental set-ups were used: (a) quiescent flow set-up and (b) crossflow set-up. The quiescent flow set-up consists of a synthetic jet generation unit mounted on the sidewall of a water tank. The suction and ejection of fluid through orifice is achieved through an oscillating diaphragm actuated using an electromagnetic actuator. The crossflow set-up is a motor driven eccentric cam that oscillates the diaphragm for generating synthetic jet. The entire mechanism of motor, cam and jet cavity has been designed to accommodate it inside the torpedo shape model having $60 \mathrm{~mm}$ outer diameter. The rpm of the motor is controlled by regulating the external power supply. The orifice is flush mounted on the curved surface of the model. For studying synthetic jet in crossflow, the model is placed inside the test section of a water tunnel.

\subsubsection{Quiescent flow set-up}

This set-up includes a transparent water tank, a synthetic jet cavity with a diaphragm, an electromagnetic actuator, and a frequency controller device. They are discussed separately in subsequent sections. 


\subsubsection{Water tank}

The water tank illuminated by laser light sheet is shown Figure 3.1. The water tank has a dimension of $1000 \times 500 \times 500 \mathrm{~mm}^{3}$ and is made of $12 \mathrm{~mm}$ thick Perspex sheet. Perspex is used to provide full optical access for characterization of synthetic jet. It is also strong enough to withstand the pressure and quite durable. The synthetic jet actuator is placed at the center of one of the sidewall of the tank (see Figure 3.1). The tank is open from top where arrangements are provided to support the traverse mechanism.

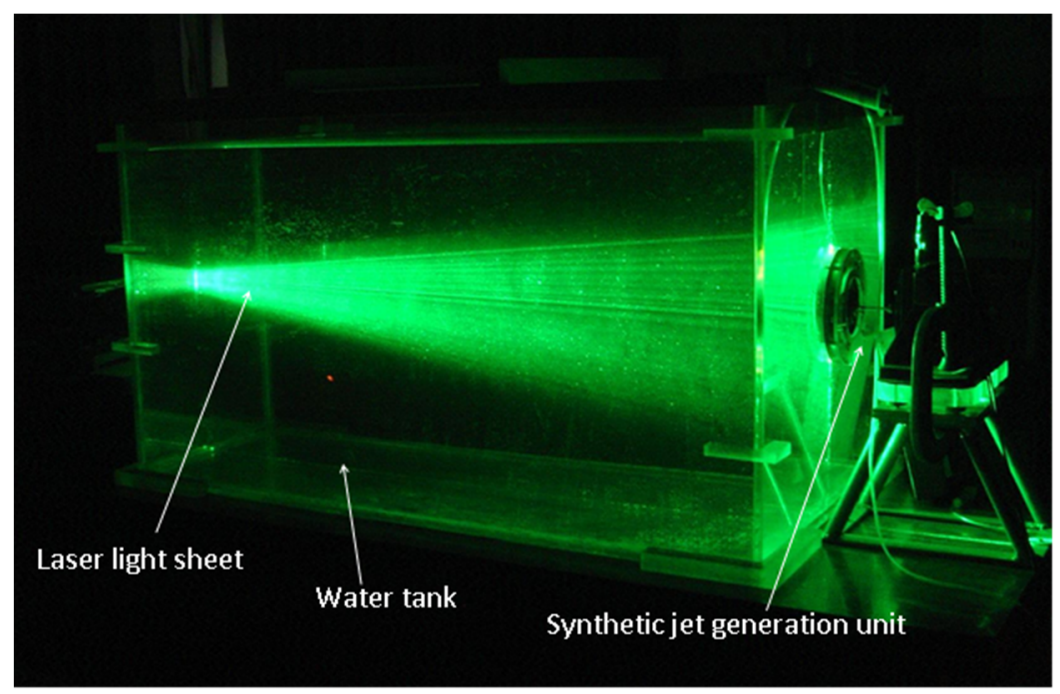

Figure 3.1: Orientation of synthetic jet and laser sheet in water tank for image acquisition in side view.

\subsubsection{Synthetic jet cavity and orifice orientation}

The schematic of synthetic jet cavity used in this study is shown in Figure 3.2 (a). It comprises of a cylindrical cavity made from Perspex, having flexible convolute type diaphragm of neoprene rubber at one of its faces, while orifice is attached on the opposite face. The inner diameter of cavity $\left(\mathrm{D}_{\mathrm{ca}}\right)$ is equal to $70 \mathrm{~mm}$ and the height $(\mathrm{H})$ is equal to 60 mm. A $3 \mathrm{~mm}$ thick (h) and $40 \mathrm{~mm}$ diameter circular steel disc $\left(D_{c y}\right)$ is fixed at the center of the diaphragm and is connected to the electromagnetic oscillating actuator using a steel rod. The cylindrical wall of synthetic jet cavity is also made of Perspex (see Figure 3.2 (a)). For dye injection, three holes of $2 \mathrm{~mm}$ diameter are drilled radially on the cylindrical surface at 
an angle $-90^{\circ}, 0^{\circ}$ and $+90^{\circ}$ with respect to the vertical axis. The orifice orientation and corresponding co-ordinate axis are shown in Figure 3.2 (b).

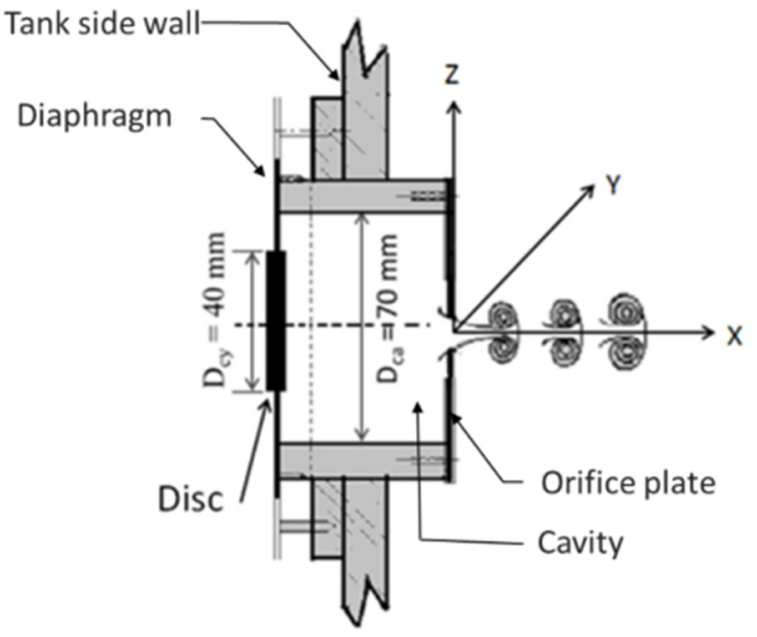

(a)

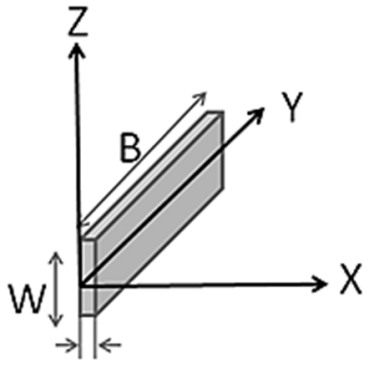

h

(b)

Figure 3.2: (a) Cross-sectional view of cavity, orifice and synthetic jet in XZ-plane and (b) coordinate notation and orifice geometry details of synthetic jet.

\subsubsection{Actuation system}


Figure 3.3: (a) Synthetic jet actuator cavity with orifice plate, (b) top view of electromagnetic actuator connected to diaphragm, (c) power supply unit with frequency and power control and (d) height adjustment and alignment base of electromagnetic actuator. 


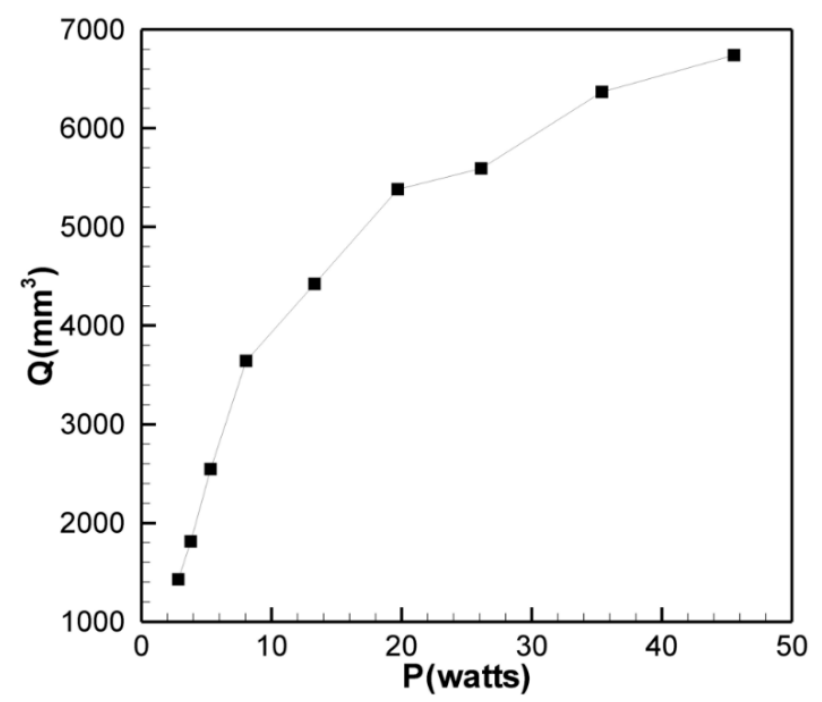

Figure 3.4: Electromagnetic actuator power (P) verses volume of fluid displaced (Q) by diaphragm during forward stroke.

The actuation mechanism of the oscillating boundary is the central part of the synthetic jet generation unit. The oscillating boundaries are mainly three types: (i) diaphragm based (ii) piston-cylinder mechanism and (iii) piezo-ceramic based membrane. However, each one having its own advantages and disadvantages. Piston driven actuators are generally heavier and contain moving parts, but they are reliable and powerful. However, they are not the optimal choice for use in confined space. The piezoelectric actuators, on other hand, are compact and consume less power but do not perform efficiently at frequencies away from the resonance frequency. The efficiency of diaphragm based actuator is unaffected by the choice of actuation frequency. They are lighter and frictionless but actuation frequency and amplitude are restricted by the property of diaphragm material. In the present experimental set-up, a diaphragm based actuation system is used.

The electromagnetic actuator shown in Figure 3.3 (b) is connected to the diaphragm through a rod which length can be adjusted. The power supply unit (Figure 3.3 (c)) supplies a sinusoidal voltage to electromagnetic actuator having adjustable peak values $( \pm 1.1-17 \mathrm{~V})$ with constant current fixed at $2.68 \mathrm{amp}$. Since the diaphragm is corrugated, deformation under the action of actuation becomes complex, because of which determination of the fluid 
expelled or displaced during forward stroke becomes difficult. However, for an electromagnetic actuator there is a relationship between the power supplied and displacement of actuating spindle connected to diaphragm irrespective of frequency. At a particular actuation frequency and power (knob) setting, the range of alternating voltage and current is measured using the oscilloscope. The voltage (sinusoidal signal) output from the frequency controller and corresponding current in ampere is measured using an oscilloscope. For the corresponding setting, the fluid displaced by the diaphragm is measured by connecting one end of a $12 \mathrm{~mm}$ diameter L-shape glass tube to the orifice. Since the frequency controller supply a sinusoidal power, fluid level in L-shape glass tube fluctuates rapidly making the measurement difficult. To circumvent this issue, a controlled constant power supply is used to actuate the electromagnetic actuator and corresponding fluid displacement is obtained by measuring the change in water level in L-shaped glass tube. The fluid displaced by the electromagnetic actuator at different power supply is shown in Figure 3.4. The others parameters are calculated as follows:

$$
\begin{aligned}
& \mathrm{V}_{\mathrm{D}}=Q_{V} ; \quad L=\frac{V_{D}}{A_{0}} ; \quad T=T_{e}+T_{s} \\
& U_{0}=V_{D} /\left(A_{0} T_{e}\right) \quad ; \quad R e=U_{0} D / v ; S_{t}=f D / U_{0}
\end{aligned}
$$

Where, $\mathrm{D}$ is orifice diameter, $\mathrm{f}$ is actuation frequency of synthetic jet, $\mathrm{L}$ is slug length, $\mathrm{A}_{0}$ is orifice cross-sectional area, $V_{D}$ is volume of fluid expelled, Re is Reynolds number (Synthetic jet), St is Strouhal number, $U_{0}$ is synthetic jet exit velocity during the forward stroke and $\mathrm{T}_{\mathrm{e}}, \mathrm{T}_{\mathrm{s}}$ forward and suction stroke time period respectively.

\subsubsection{Crossflow set-up}

A torpedo shape model with a built-in synthetic jet generation unit is fabricated to study the behavior of synthetic jet in crossflow environment. The crossflow is obtained by putting the model inside the test section of a water tunnel. The dimensions of test section of water 
tunnel impose space constraint to the model. A term blockage ratio is used to identify the blockage created by the model when placed inside the test section, and is defined as the ratio of model to test section cross-sectional areas. The blockage ratio provides an estimation of changes in local fluid velocity around the model. The effect of blockage ratio is more serious at low Reynolds number flow and hence it is advisable to keep the blockage ratio as low as possible. The cross sectional area of the test section is $400 \mathrm{~mm} \times 400 \mathrm{~mm}$ while the diameter $\left(\mathrm{D}_{\mathrm{m}}\right)$ of the model is $60 \mathrm{~mm}$. With this dimension, the blockage ratio based on cross sectional area is calculated to be 0.017 , which is within the acceptable range.

The detail of the model is discussed in following five sections: (a) motor with its power supply and control unit, (b) diaphragm with actuation mechanism, (c) synthetic jet cavity and orifice, (d) assembly of components and (e) model support system.

\subsubsection{Motor and control unit}

A three-phase DC brushless motor (42RBL85) is used for rotating the cam. The motor uses hall-sensors feedback system to control the speed of the motor. The rated torque of the motor is $0.15 \mathrm{Nm}$ at $4000 \mathrm{rpm}$. The approximate motor weight is $4 \mathrm{~N}$ with maximum power rating of 96 watts. The safe current for motor and power drive board is 4 amps. The outer diameter of motor is $42 \mathrm{~mm}$ while the length is $90 \mathrm{~mm}$. The power supply unit converts single-phase 220 -volt AC into 18 volts DC. The controller using a multi-turn potentiometer sets the upper limit of power ranging between 0 - 96 watts. The motor is connected to a power drive board (AMB364) which converts a single-phase DC power supply coming from controller into three-phase DC before supplying to the motor as shown in Figure 3.5. 

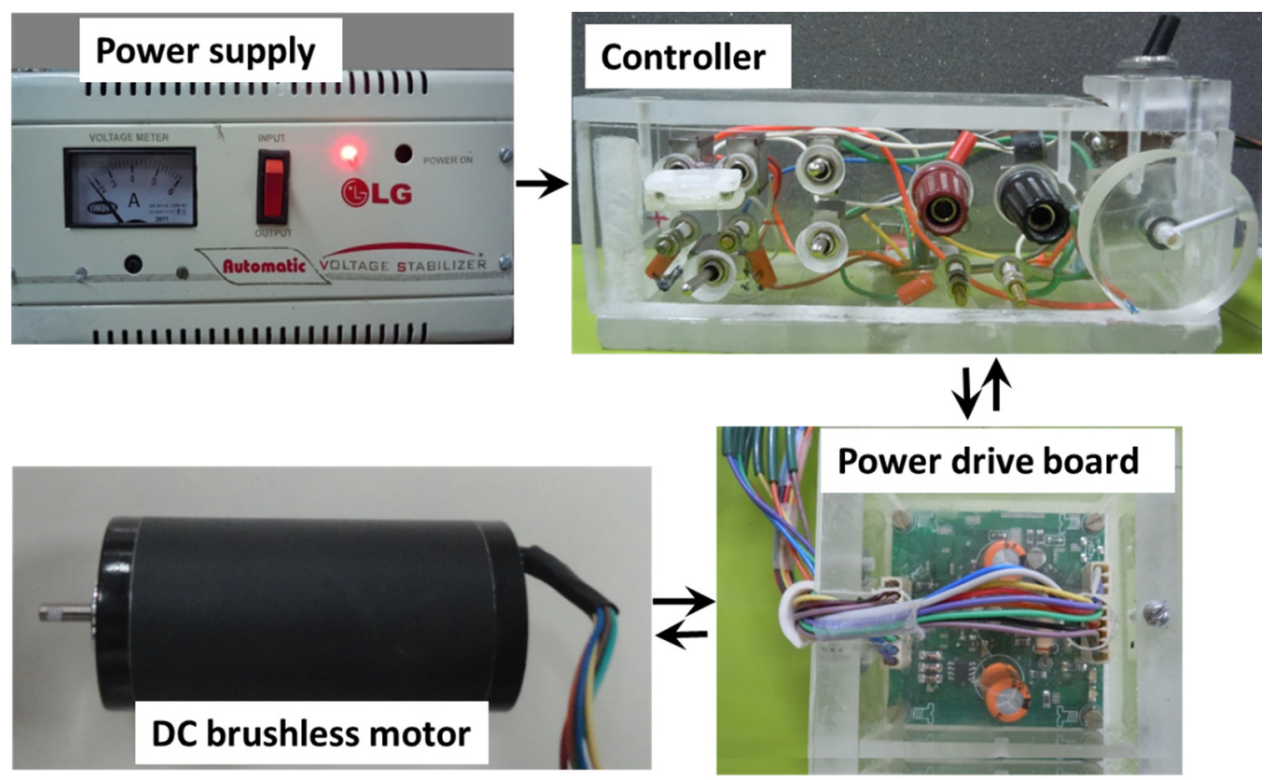

Figure 3.5: Power supply, controller, power drive board and DC brushless motor for synthetic jet built-in in torpedo shaped model.

The power drive board generates a train of TTL (Transistor-Transistor-Logic) pulses according to the motor rotation using hall sensors. There are five hall sensors mounted on the periphery of rotating shaft at the interval of $72^{\circ}$. Therefore, one revolution of motor shaft generates five TTL pulses. The number of TTL pulses are counted using an oscilloscope, whereas the frequency is measured using a tachometer. Since the cam is mounted to the motor shaft, each rotation of motor shaft gives back-and-forth motion to the diaphragm that generates a vortex ring when fluid is ejected out of the orifice. Therefore, the actuation frequency of the diaphragm is found to be $1 / 5^{\text {th }}$ of the total number of TTL pulses in one second. The cam angular speed is observed to be non-uniform for actuation frequency less than $2 \mathrm{~Hz}$ and hence, the duration between two consecutive TTL pulses is found to be larger during the forward stroke than that of backward stroke as shown in Figure 3.9 . 


\subsubsection{Synthetic jet cavity}

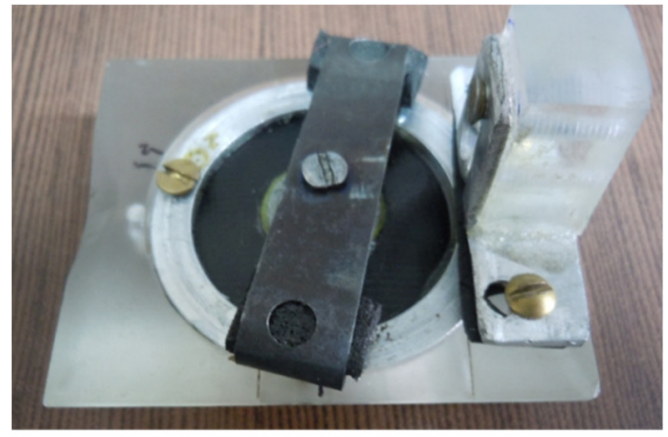

(a) Back view of diaphragm

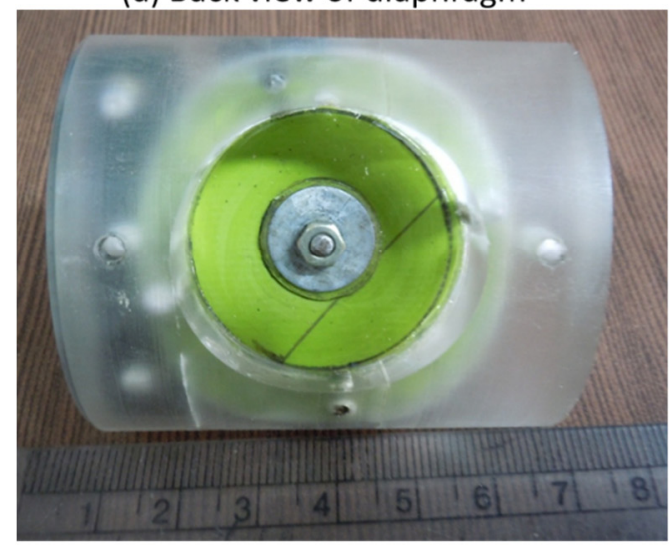

(b)Front view of diaphragm

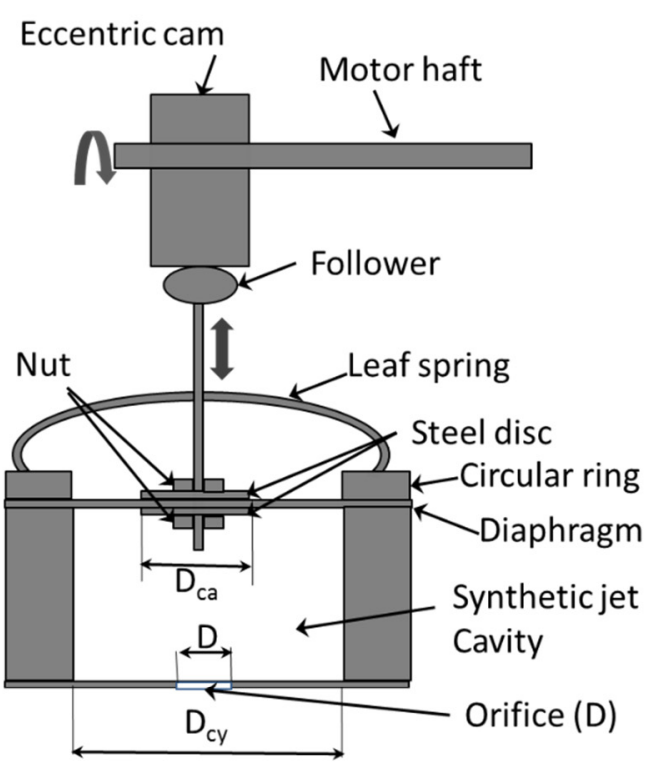

(c) Pictorial representation of cam-follower mechanism to oscillate the diaphragm

Figure 3.6: (a) Back view of diaphragm connected to cam follower, (b) front view of diaphragm with cavity and (c) schematic of synthetic jet generation mechanism.

The cavity, which has the diaphragm at one end and orifice on the other end, is made from

Perspex. The diaphragm is put in place using three circular disks having a thickness of 20 $\mathrm{mm}$ and diameter of $54 \mathrm{~mm}$. The cavity also houses the actuation mechanism that uses a diaphragm, steel discs and a leaf spring on one end. A plane circular plate with a central hole acts as an orifice on the other end.

\subsubsection{Diaphragm and actuation mechanism}

The selection of diaphragm material is important due to the following reasons: (i) a continuous cyclic force causes tearing of diaphragm, (ii) lower flexibility increases the force requirement and (iii) larger size results in more recovery time thus breaking the contact between cam and follower at higher actuation frequency. Considering all the above limitations, after a number of trials, the diaphragm is finally made using $0.46 \mathrm{~mm}$ thick 
nitrile coated nylon rubber sheet (see Figure 3.6 (b)). To avoid tearing near the screw where holes are punched, the diaphragm is glued permanently over a $1.5 \mathrm{~mm}$ thick circular aluminum ring having inner and outer diameter equal to $33 \mathrm{~mm}$ and $43.5 \mathrm{~mm}$ respectively. A leaf spring made from $0.5 \mathrm{~mm}$ thick and $15 \mathrm{~mm}$ wide carbon steel strip as shown in Figure 3.6 is used to ensure a continuous contact between the cam and the follower.

\subsubsection{Assembly of components}

The exploded views of model components are shown in Figure 3.7 (a). The overall model length is $260 \mathrm{~mm}$ and diameter is $60 \mathrm{~mm}$. The major components are hull, motor with its eccentric casing, back side cover disk, synthetic jet cavity with diaphragm, cam-follower, front cover and nose. The motor is kept eccentric inside the cavity to have more room for cam-follower mechanism. The cam is mounted on the shaft of motor and then inserted inside the eccentric cavity. The backside is covered with aluminum plate having sealing rubber in the circular grooves.

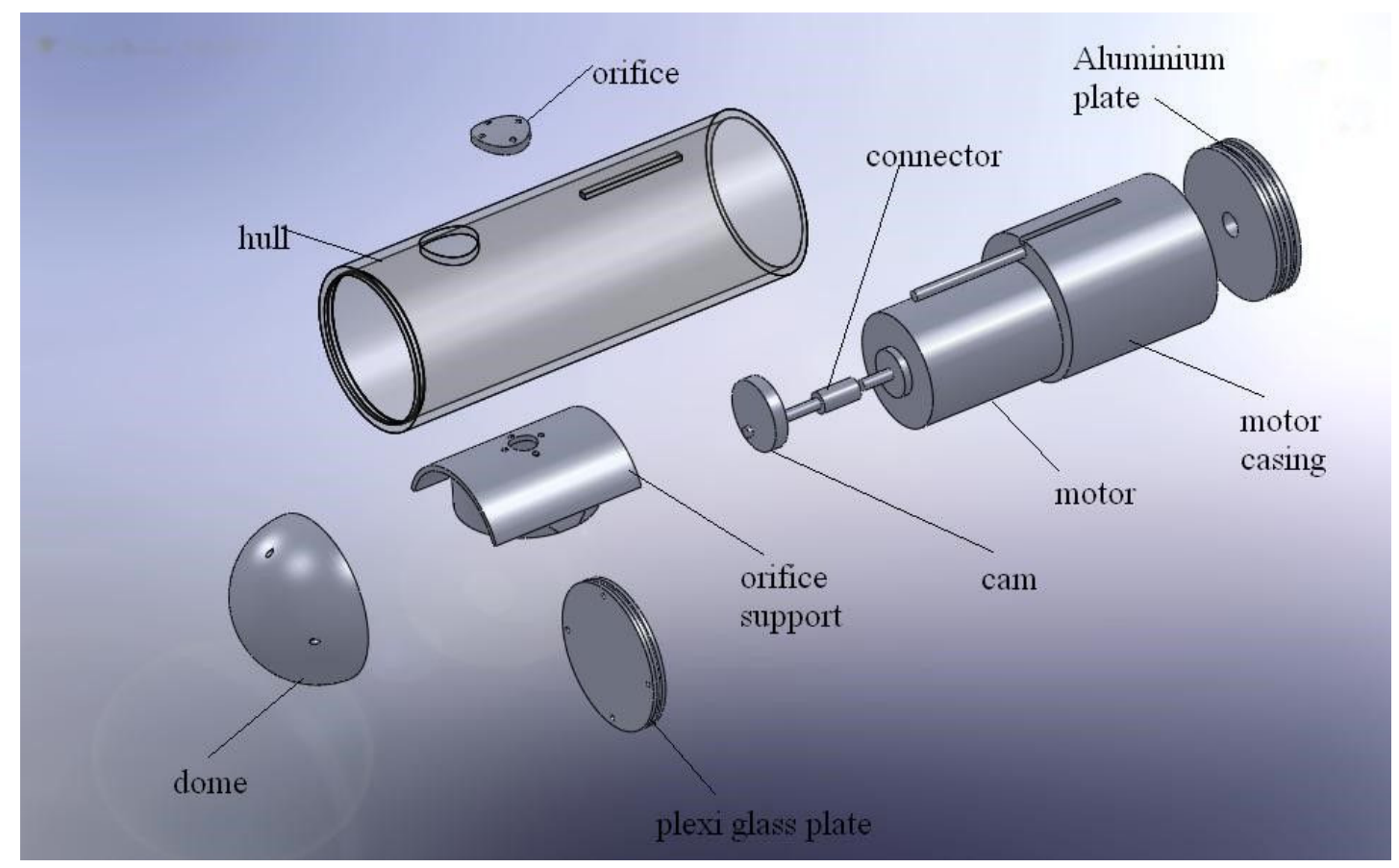

(a) 


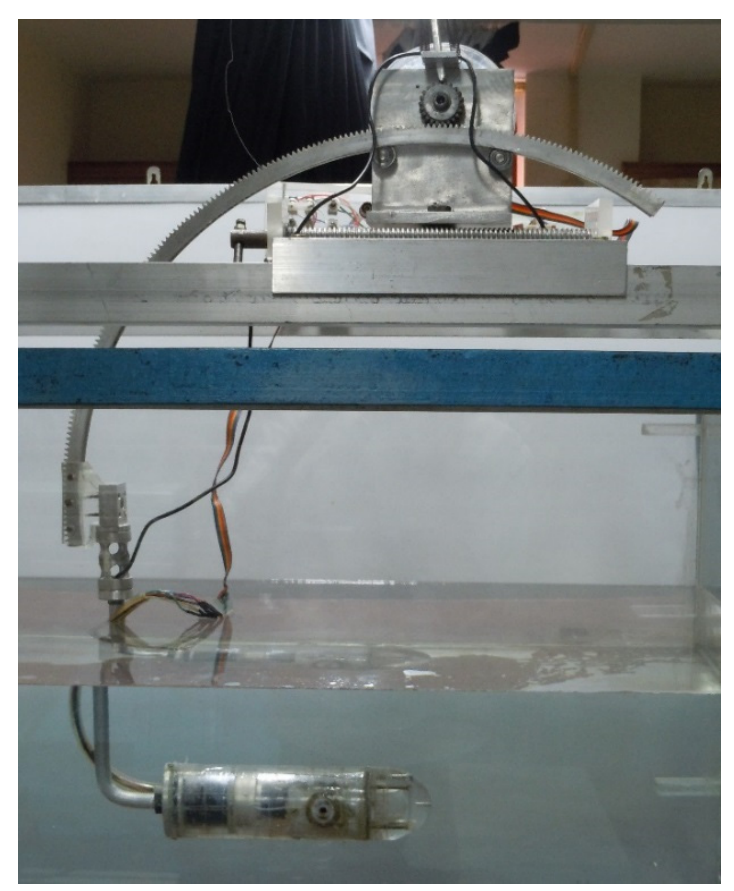

(b)

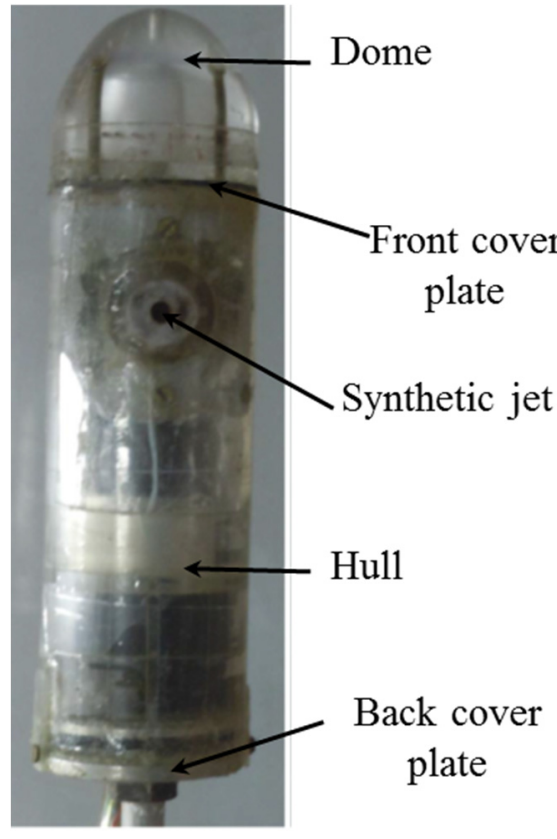

(c)

Figure 3.7: (a) Exploded view of torpedo shape model with built-in synthetic jet, (b) model in test section of water tunnel and (c) assembled view of model.

\subsubsection{Dynamic model support system}

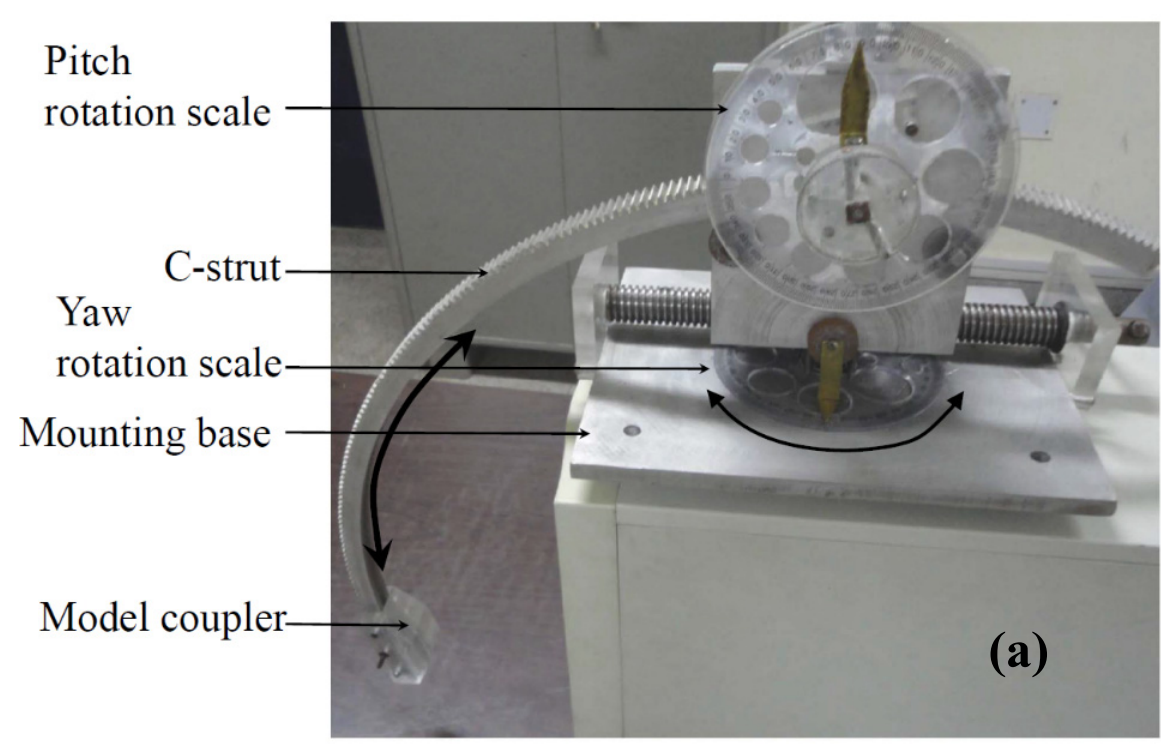




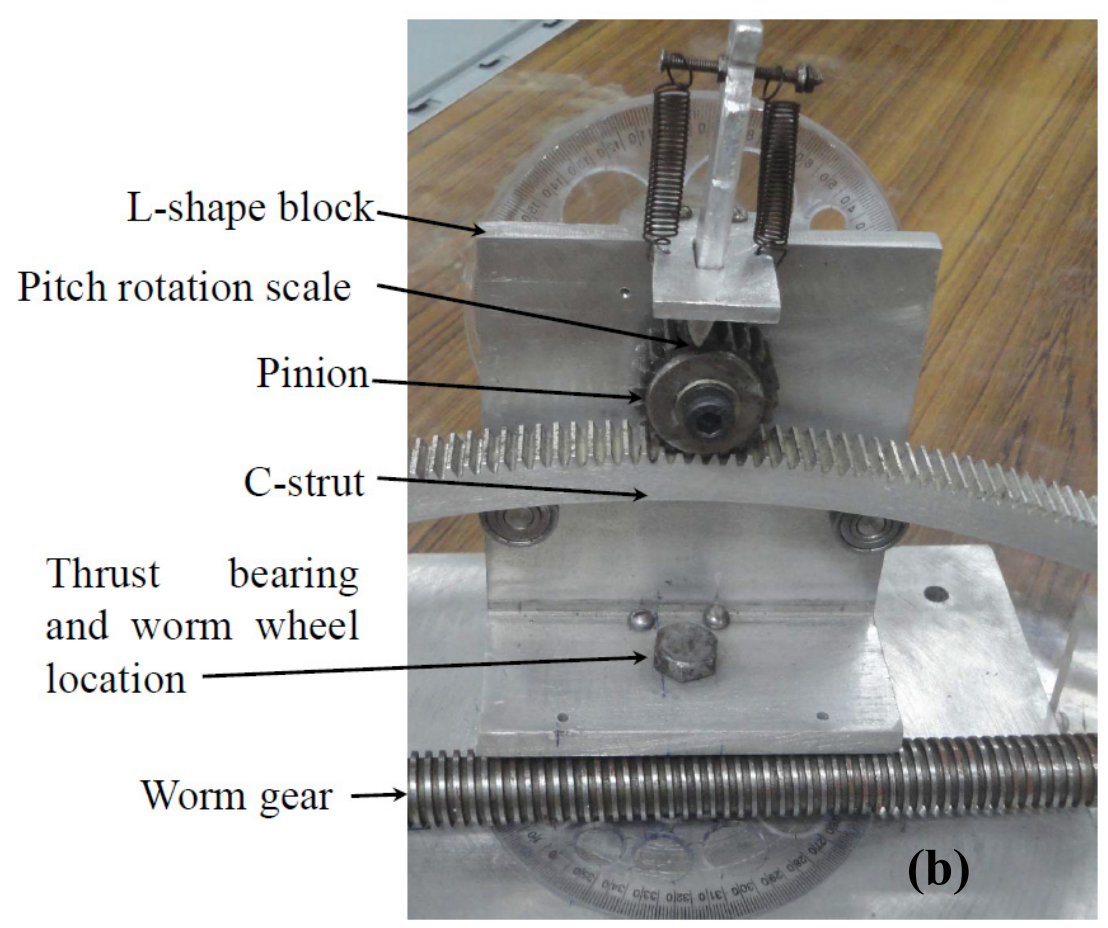

Figure 3.8: (a) Front and (b) back view of dynamic model support system.

A proper model support system called as dynamic model support system is designed and fabricated to vary yaw and pitch angles of the model and is shown in Figure 3.8. The model is mounted at one of the ends of $\mathrm{C}$-strut. For achieving pitching of the $\mathrm{C}$-strut, the dynamic model support system works on a gear-pinion mechanism (see Figure 3.8 (b)). Gear teeth have been provided on the $\mathrm{C}$-strut, so that it can act as a part of gear. The $\mathrm{C}$-strut has groove on its inner circumference to allow sliding motion over two thrust bearings in the vertical plane for pitching motion. A separate pinion is engaged to the C-strut gear using which the pitching angle is set during the experiments. Due to weight of the model, C-strut rotates automatically in downward direction. The complete pitching unit is fixed on the longer side of an L-shaped aluminum block. The shorter side of the aluminum block is fixed to the horizontal floor of the support system with the help of thrust bearings and a wheel. The yaw movement is given to the support system by rotating the wheel using a worm gear. The rotation angle is measured by a pointer sliding over the protector. Each degree on 
protector gives $0.05^{\circ}$ degree of rotation to the model in yaw plane. The model mounted on model support system is shown in Figure 3.7 (b).

\subsubsection{Actuation frequency and slug length}

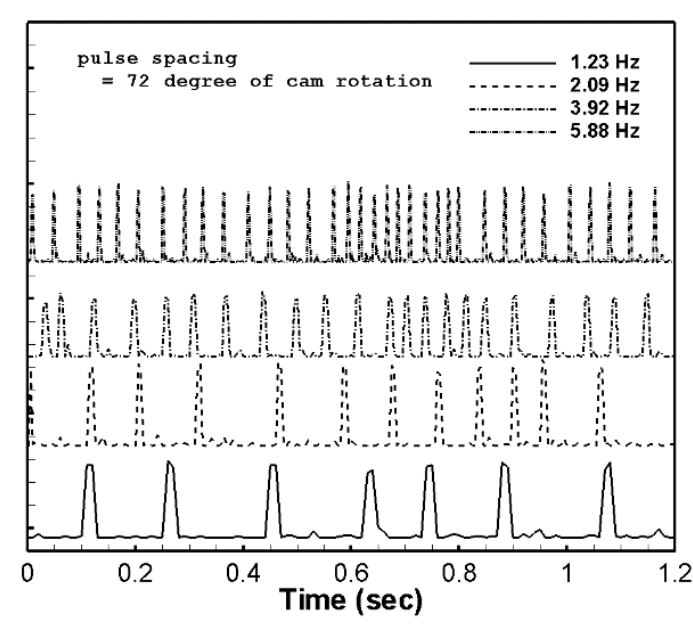

(a)



(b)

Figure 3.9: (a) Actuation frequency obtained from pulse generated by sensors mounted at interval of 72 degree inside the motor and (b) flow frequency obtained from FFT of jet exit velocity. Plots are shifted in $\mathbf{Y}$-axis for clarity.

The actuation frequency of synthetic jet i.e., the cam rotation is regulated using the motor control unit. Each rotation of cam produces a reciprocating motion of the spindle attached to the diaphragm thus completing one full cycle of synthetic jet. The displacement of diaphragm $(\Delta)$ during each cycle of synthetic jet is equal to twice the eccentricity given to the cam. In Figure 3.9 (a), actuation frequency (f) is measured from the sensor signals in the form of TTL pulses generated by the motor. The corresponding flow frequency is obtained by performing Fast Fourier Transformation (FFT) of velocity signal captured using LDV at the center of the jet exit.

In Figure 3.9 (a), the differential of TTL pulse signal is shown while the flow frequency obtained by computing the FFT of jet velocity signal are shown in Figure 3.9 (b) 
Table 3.1: Actuation frequency of cam operated synthetic jet

\begin{tabular}{|c|c|c|c|}
\hline S.N. & $\begin{array}{c}\text { Actuation frequency calculated from } \\
\text { the pulse of hall sensors }\end{array}$ & $\begin{array}{c}\text { Actuation frequency calculated using } \\
\text { velocity signal }\end{array}$ & $\begin{array}{c}\mathbf{( \% )} \\
\text { error }\end{array}$ \\
\hline 1 & 1.23 & 1.19 & 3 \\
\hline 2 & 2.09 & 2.14 & 2 \\
\hline 3 & 3.92 & 4.11 & 5 \\
\hline 4 & 5.88 & 5.83 & 1 \\
\hline
\end{tabular}

For a sinusoidal profile, the ejected volume $\left(V_{D}\right)$ for an oscillating diaphragm system (shown in Figure 3.6) is estimated using cavity inner diameter $\left(\mathrm{D}_{\mathrm{ca}}\right)$, disk diameter $\left(\mathrm{D}_{\mathrm{cy}}\right)$ and amplitude of oscillation $(\Delta)$ as the following equations (Mohseni, 2006).

$$
\mathrm{V}_{\mathrm{D}}=\frac{\pi \Delta}{8}\left(D_{c a}^{2}+D_{c y}^{2}\right) ; \quad V_{R}=U_{0} / U_{\infty}
$$

Where, $\mathrm{D}_{\mathrm{ca}}=33 \mathrm{~mm}, \mathrm{D}_{\mathrm{cy}}=15 \mathrm{~mm}, \mathrm{D}=6 \mathrm{~mm}, \mathrm{~h}=6 \mathrm{~mm}, \Delta=1.5$ and $2 \mathrm{~mm}$ for inbuilt synthetic jet in torpedo shape model. The other parameters are calculated using the Equation 3.2 and 3.3. In the above expressions, $V_{R}$ is velocity ratio and $U_{\infty}$ is the crossflow velocity. 


\subsection{Measurement techniques}

In order to measure the synthetic jet velocity quantitatively, two techniques namely (a) hotfilm anemometer and (b) Laser Doppler velocimetry are used. The hot-film anemometer uses an L-shaped single film operating on Constant Temperature Anemometry (CTA) mode. The Laser Doppler Velocimetry (LDV) has single probe with beam emitter and back scattering sensors (also called trans-receiver) and is capable of measuring all three components of velocity. On the other hand, for qualitative analysis, Laser induced fluorescence (LIF) and bulk flow dye visualization technique has been employed for flow visualization. A brief introduction of each technique, experimental set-up employed and other details related to experiments are provided in the following sections.

\subsubsection{Hot-film anemometry}

Hot-film is extensively used for velocity measurement of moving fluid because of its higher frequency response in the range of 0 to $1 \mathrm{MHz}$, wide velocity measuring range, low signal to noise ratio (1:10000) and higher sensitivity to velocity fluctuation. The velocity of moving fluid is measured by continuous analog signal generated by hot-film placed at the desired location in flow field. Using calibration curve (voltage v/s velocity), the analog electrical signal is then converted into velocity signal. Since the measured velocity is sampled at fixed time interval, spectral or time-frequency analysis of velocity signal becomes easier. However, it is an intrusive technique and modifies the local flow field. The deposition of impurities on sensors alters the calibration curve and hence it performance degrades with time. The fragile nature of the probe makes it unsuitable for hostile environment and sensitiveness to environmental conditions requires recalibration or correction to the calibration curve for each experiment. There are two modes of hot-film operations: (a) Constant Temperature Anemometry (CTA) and (b) Constant Current Anemometry (CCA). 
In CTA mode, the hot-film sensor is a part of the one resistance out of four resistances in a Wheatstone bridge and is heated by an electrical current to a pre-defined constant temperature. The bridge is balanced by controlling the current to the sensor, so that the resistance and consequently temperature is kept constant, independent of the cooling imposed by the flowing fluid. The bridge voltage represents the amount of heat transfer from wire due to fluid flow and thus a direct measure of the fluid velocity is possible. On the other hand, in CCA mode, a constant current is maintained through the bridge. The wire resistance changes due to heat convected by the flowing fluid resulting in drop in voltage across the wire. In this mode of operation, if the velocity is too low, there is a possibility of burning out of wire and the cyclic change of wire-temperature reduces the life of the hot-film, which is not present in CTA mode. The King's law proposed an empirical relationship between the voltage drop (E) and fluid velocity (U) by considering heat transfer from the wire through convection and conduction and is expressed as

$$
I^{2} R_{w}^{2}=E^{2}=\left(T_{w}-T_{a}\right)\left(A+B U^{n}\right)
$$

where $T_{w}$ is wire temperature, $I$ is heating current, $T_{a}$ is the temperature of the fluid and $R_{w}$ is the wire resistance. The constants $\mathrm{A}, \mathrm{B}$, and $\mathrm{n}$ are experimentally determined and ambient specific. For CTA, the wire temperature and ambient temperature is remains constant and can be incorporated into $\mathrm{A}$ and $\mathrm{B}$ then equation (3.4) reduces to

$$
E^{2}=\left(A+B U^{n}\right)
$$

Before measuring the velocity, a calibration is required to know the constants $\mathrm{A}, \mathrm{B}$ and $\mathrm{n}$. In the present study, a calibration unit measuring the velocity of potential core of a water jet is developed and used to calibrate the S-type hot-film. The detail of calibration set-up and calibration procedure is given in appendix-B. 


\subsubsection{Experimental set-up for hot-film measurement}

The velocity measurements are carried out using single film probe (TSI 1212-20W), connected to a constant temperature anemometry system (TSI, Model No: IFA 300). A Ttype thermocouple is mounted inside the water tank and is connected to the anemometry system for temperature correction. Data is acquired at $1 \mathrm{kHz}(1000 \mathrm{samples} / \mathrm{sec})$ and is passed through the low pass filter (set at $500 \mathrm{~Hz}$ ) to eliminate the high frequency noise from the signal. The data acquisition is carried out using software THERMAL-PRO (TSI). A self-developed LABVIEW program is used for further post-processing of the data. A three-dimensional traverse is used for moving the hot-film in three different directions. The distilled water is used as working fluid to avoid contamination and fouling of hot-film. The room temperature is kept within $\pm 1{ }^{\circ} \mathrm{C}$ of the calibration temperature to minimize the influence of environment temperature on the measurement and corrections are made wherever necessary.



Figure 3.10: Schematic of hot-film calibration set-up. 


\subsubsection{Laser Doppler Velocimetry}

The hot-film technique for measuring velocity is complicated and profoundly depends on environment condition such as temperature and turbidity of water. In addition, particularly at very low velocity $(0.0001$ to $0.05 \mathrm{~m} / \mathrm{s})$, the measured velocity is highly unstable as a small change in ambient condition significantly alters the measured value. The major disadvantage that prompts us to use LDV is the quality of water. The water tunnel requires a large amount of water ( $\sim 4000$ liters) and it is practically very difficult to maintain rust and dust free environment in the water tunnel.

The principle of operation of LDV for measuring the fluid velocity is based on Doppler Effect. The Doppler Effect in fluid flow is caused by moving tiny particles suspended in water results in interference fringes of laser beams. The particles could be of different materials but they must have relative refractive index different from the moving fluid. Micron sized particles when dispersed in the fluid, they become non-buoyant and velocity of particles can be assumed equal to the velocity of the fluid itself. Depending on the relative motion between the light and particle, the light wave is either compressed or expanded. This effect or shift in frequency of light wave is known as Doppler shift. The change in frequency of light wave can be obtained by comparing it with the reference wave. Since the frequency of light wave is high as compared to the Doppler shift and it becomes difficult to measure directly. Hence, a dual-beam or fringe method is used in order to calculate it. The another advantage of dual-beam method over reference beam method is detection of flow reversal or negative velocity using Bragg's cell or right angle polarization flow. In dual-beam method, velocity component opposite to the fringe motion is treated as positive, whereas it is considered negative when the velocity component is along the fringe motion (see Figure 3.11). However, for flow having higher particle density producing multiple scattering of light, reference beam method gives more accurate measurement than 
dual-beam method. Since the present experiments use dual-beam method, the subsequent sub-sections cover discussions on only dual-beam method.

\subsubsection{Measurement volume}

The intersection of two laser beams of same wavelength forms a fringe pattern as shown in Figure 3.11 (a) and (b). The fringe pattern forms ellipsoidal shape of waist diameter $\left(\mathrm{d}_{\mathrm{m}}\right)$ and length $\left(\mathrm{l}_{\mathrm{x}}\right)$. The waist diameter and length depends on the focal length of achromatic lens. For $350 \mathrm{~mm}$ achromatic lens used in the laser gun, the waist diameter $\left(\mathrm{d}_{\mathrm{m}}\right)$ and length $\left(1_{\mathrm{m}}\right)$ are found to be $145 \mu \mathrm{m}$ and $4.2 \mathrm{~mm}$ respectively for wavelength $514.5 \mathrm{~nm}$ and $137 \mu \mathrm{m}$ and $2 \mathrm{~mm}$ for wavelength $488 \mathrm{~nm}$. The fringe spacing and number of fringes are found to be $7.47 \mu \mathrm{m}$ and 19 for wavelength $514.5 \mathrm{~nm}$ while for wavelength of $488 \mathrm{~nm}$ they are 3.54 $\mu \mathrm{m}$ and 39 respectively. A moving particle crossing the measuring volume scatters light and no light as it travels past a bright and dark fringes respectively. Each peak in the burst signal represents the scattered light intensity of individual bright fringe (see Figure 3.11 (c)). The alternate bright and dark fringes give a fluctuating pattern of scattered light intensity with a frequency proportional to the particle velocity. Since the laser beam intensity follows Gaussian profile, intensity of fringes also follows the same profile and consequently produces a burst signal having a Gaussian distribution. When the scattered light intensity is below threshold intensity, it is discarded to reduce the noise or ambiguity in the signal. The strength or the intensity of burst signal is higher at the center and then gradually decreases outwards. When intensity threshold is applied, the apparent waist diameter and length of measuring volume for sensors reduces as shown by a circle in Figure 3.11 (b). 


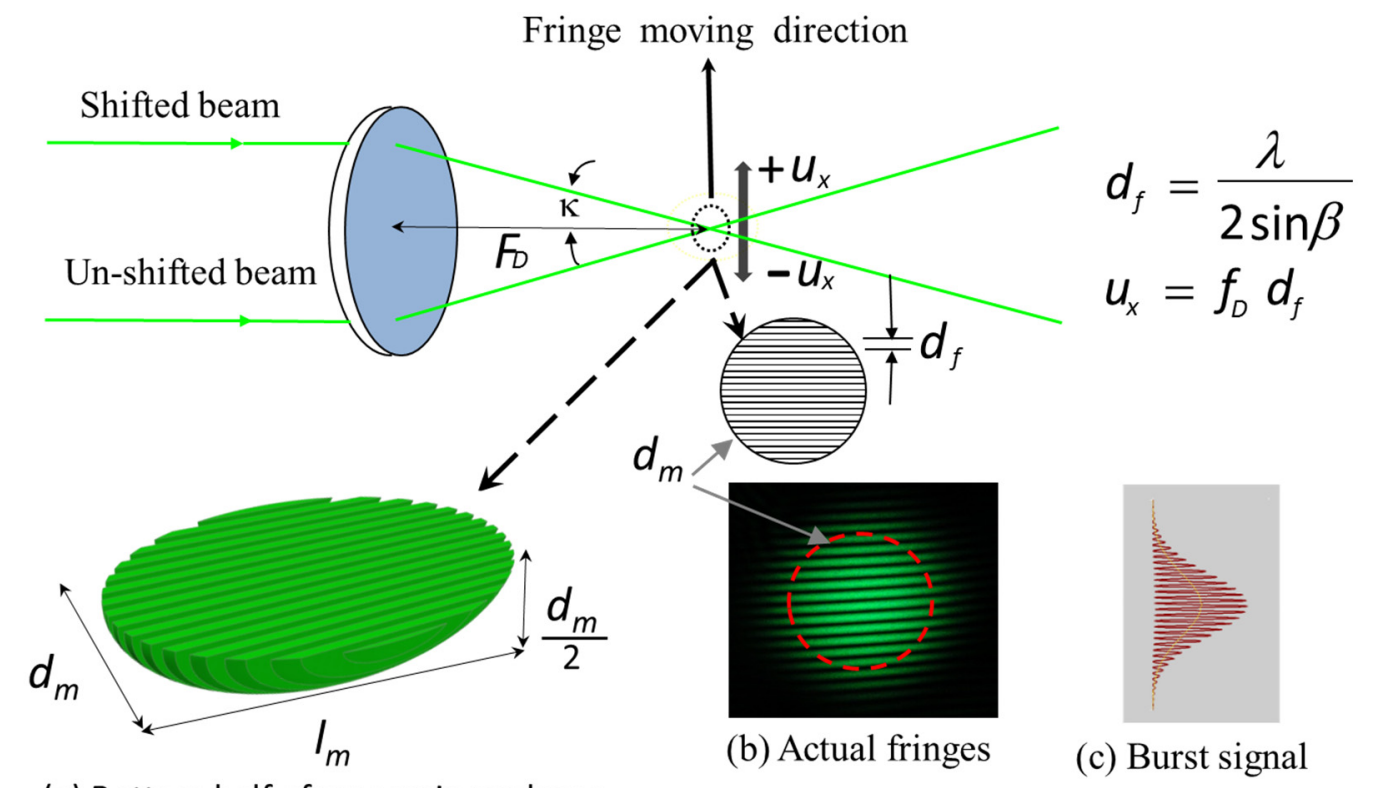

(a) Bottom half of measuring volume

Figure 3.11: (a) Cross sectional view of measuring volume, (b) zoomed view of actual fringe and (c) burst signal when a particle crosses the fringe pattern. (* Figure (b) and (c) are taken from the TSI manual)

\subsubsection{Velocity reduction}

The velocities of moving particles are calculated from the frequency of scattered light. The scattered light is collected by receiving optics and then converted into electrical signals known as burst signal by photomultiplier tubes (PMTs). The distance between fringes $\left(\mathrm{d}_{\mathrm{f}}\right)$ is calculated from known values of wavelength $(\lambda)$ and half angle $(\beta)$ as shown in Figure 3.11. The fringe spacing is a system dependent quantity varying with wavelength of laser light source and beam spacing at focusing lens of probe. The dual-beam LDV method can accurately measure the magnitude of velocity component but velocity reversal cannot be detected, as the change of sign has no effect on Doppler frequency (fD). To overcome this, frequency of one of the beams is shifted using acoustic optical modulator commonly known as Bragg cell. This makes the fringes to move in the measuring volume at the rate of shifted frequency $\left(\mathrm{f}_{\mathrm{B}}\right)$ and this frequency shift is called as Bragg shift and the process is called as down-mixing. The resultant frequency $\left(\mathrm{f}_{\mathrm{R}}\right)$ of the signal is expressed as: 


$$
f_{R}=f_{B} \pm f_{D}
$$

Therefore, if a particle moves against the motion of fringes, it will have a frequency equal to shifted frequency plus the Doppler frequency and if the particle moves in the direction of fringes, it will have a frequency equal to the shifted frequency minus the Doppler frequency. If the resultant frequency of burst signal is less than the shifted frequency, velocity is negative whereas for positive velocity, the resultant frequency is higher in comparison to the shifted frequency. The output of PMT is first high-pass filtered to remove the low frequency (pedestal) noise caused by the particles passing through the beam waist. Signal is then passed through selected series of band pass filters to eliminate the high frequency noise as well as the brag shift frequency. Once the Doppler frequency is obtained using equation 3.6, the velocity component is calculated by using following expression.

$$
u=f_{D} * d_{f}
$$

\subsubsection{Refractive index (window) correction}

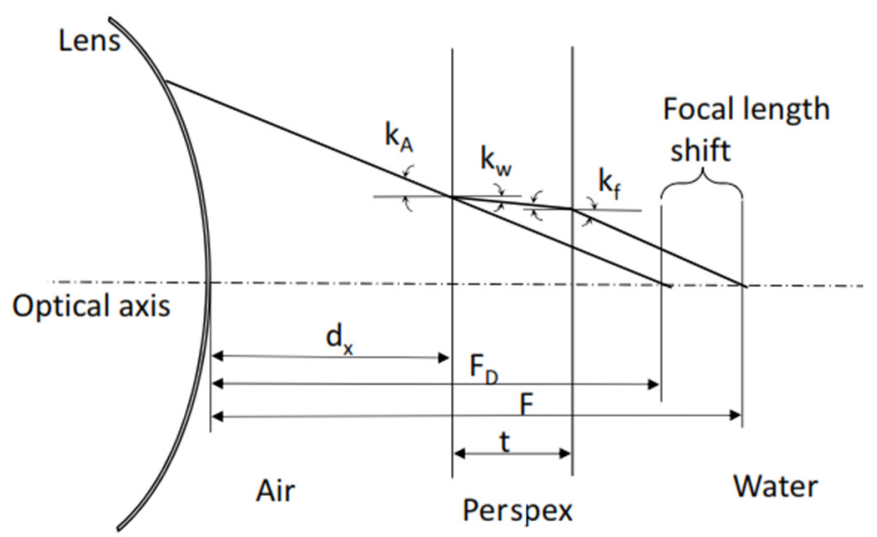

Figure 3.12: Deviation in the path of laser beam due to changes in refractive index of optical medium.

The laser beam propagates through different mediums before the scattered light traveling back to receiving optical sensors, after forming the fringe pattern. The changes in refractive index of optical medium change the path of laser beams. Both the fringe spacing $\left(\mathrm{d}_{\mathrm{f}}\right)$ and 
apparent focal length of lens depend on the path of laser beam. Depending on relative refractive index of optical mediums, the apparent focal length of laser beam increases or decreases. The fringe width $\left(\mathrm{d}_{\mathrm{f}}\right)$ as expressed in equation 3.8 remains unaffected as the ratio of wavelength to sine function of half angle $(\beta)$ remains unchanged irrespective of medium.

$$
d_{f}=\frac{\lambda_{f}}{2 \sin \beta_{f}}=\frac{\lambda_{A} / N_{f}}{2\left(\sin \beta_{A}\right) / N_{f}}=\frac{\lambda_{A}}{2 \sin \beta_{A}}
$$

In the above expression, $\lambda_{\mathrm{A}}$ and $\lambda_{\mathrm{f}}$ are the wavelength of laser light in air and water respectively. Similarly, $\beta_{\mathrm{A}}$ and $\beta_{\mathrm{f}}$ are the half angle of the incident light in air-Perspex and Perspex- water interface. However, the apparent focal length, which is the distance between the lens and measurement point is influenced by the medium of light propagation. So, whenever the probe is moved along its axial direction, the apparent focal length must be corrected. The laser beam propagation and deviation from its original path due to refractive index variation is shown in Figure 3.12.

Three different optical mediums such as air, Perspex, and water are present in the current experiments. The thickness ( $\mathrm{t}$ ) and refractive index (n) of Perspex sheet are found to be $12 \mathrm{~mm}$ and 1.49 respectively. The refractive index of water in the tank and air is 1.33 and 1.0 respectively. The incident angles $\mathrm{k}_{\mathrm{a}}$ and $\mathrm{k}_{\mathrm{w}}$ and reflected angles $\mathrm{k}_{\mathrm{w}}$ and $\mathrm{k}_{\mathrm{f}}$ are shown in Figure 3.12. The incident angle at air-Perspex interface $\left(\mathrm{k}_{\mathrm{a}}\right)$ is calculated using beam offset from the axis of achromatic lens and $\mathrm{d}_{\mathrm{x}}, \mathrm{k}_{\mathrm{w}}$ and $\mathrm{k}_{\mathrm{f}}$ is calculated using Snell's law. Finally, the apparent focal length $(\mathrm{F})$ is calculated using the expression below:

$$
F=F_{D} \frac{\tan k_{A}}{\tan k_{f}}+t\left[1-\frac{\tan k_{w}}{\tan k_{f}}\right]+d_{x}\left[1-\frac{\tan k_{A}}{\tan k_{f}}\right]
$$

\subsubsection{Seeding particles}

Seeding particles are an essential part of a LDV system. In general, seeding particles are inherent to any fluid flow system because of impurities present in the fluid. However, in 
the cases where the particle density is low or the intensity of scattered light from the natural particles are weak, external seeding is needed. In laboratory, it is in common practice to seed the flow externally to achieve sufficient scattering intensity and large number of particles crossing the fringes. The scattered light intensity received by the photo-detector sensors must be sufficient to allow reliable processing of the signal by the subsequent electronic unit. The ability of a particle to follow the fluctuations in the flow depends on the particle's aerodynamic diameter $\left(\mathrm{d}_{\mathrm{a}}\right)$ expressed as

$$
d_{a}=d_{g} \sqrt{\rho_{p}}
$$

The relaxation time $\left(\tau_{\mathrm{s}}\right)$ is a convenient measure to determine the velocity equilibrium of scattering particle with the flowing fluid. Assuming that particles are spherical, Stokes's drag law gives

$$
\tau_{s}=d_{a}^{2} /(18 \mu)
$$

where $d_{g}$ is particle's geometric diameter, $\rho_{p}$ is particle density and $\mu$ is dynamic viscosity of fluid. A smaller aerodynamic diameter provides a higher frequency response and a greater ability to follow rapid flow fluctuations. Another important characteristic of scattering particle is intensity and observation angle. If the particle diameter is larger than the wavelength of light source, then Mie's scattering theory is applied. The scattered light intensity is proportional to the square root of ratio of particle diameter to the wavelength of light. From experimental observations, it is found that particles of $10-12 \mu \mathrm{m}$ diameter are best suitable for water. The seeding particles used in the present experiment are silvercoated hollow glass spheres having diameter of $8-12 \mu \mathrm{m}$. 


\subsubsection{Experimental set-up for LDV}
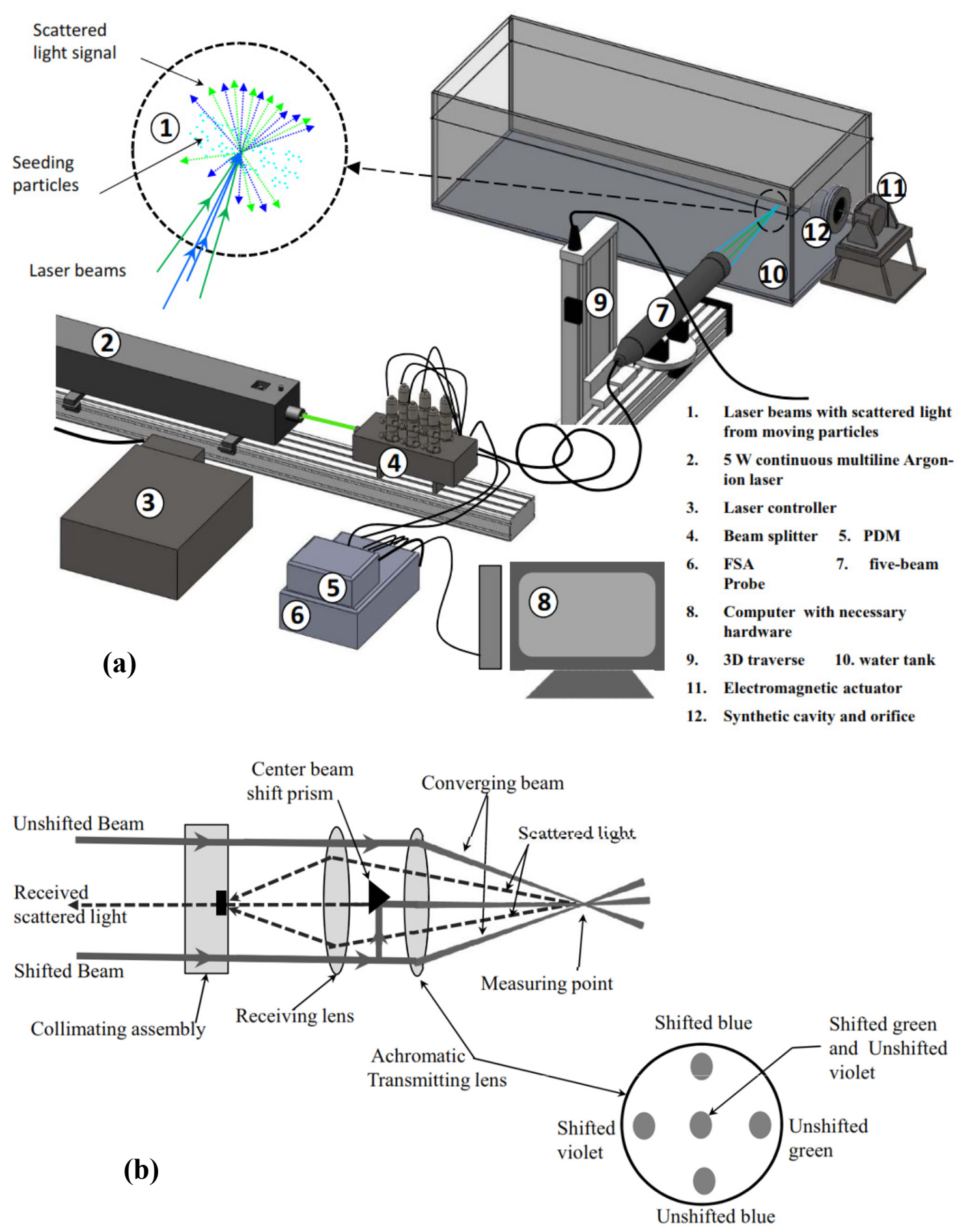

Figure 3.13: Schematic of experimental set-up for Laser Doppler Velocimetry for (a) laser beam propagation inside the 5-beam probe gun and (b) front view of achromatic lens showing positions of all six beams.

The experimental arrangements for velocity measurement of synthetic jet in quiescent flow using LDV is shown in Figure 3.13 (a). A LDV system consists: (a) laser source, (b) 
transmitting and receiving optics and (c) signal conditioner and processing units. In order to have a sharp diffraction pattern with bright fringes, beams must be of equal intensity, coherent, and exhibit the same polarization. For this purpose, a continuous argon ion laser (5W Innova 70C-5) is used as a laser source. The emitted laser beam has diameter equal to $1.5 \mathrm{~mm}$ with Gaussian intensity profile and beam divergence equal to 1.2 radians per meter. The transmitting and receiving optics system consist a multicolor beam generator, fiberoptic couplers and transmitting-receiving probe. The multicolor beam generator separates the laser beam into three different beams of wavelength of $514.5 \mathrm{~nm}$ (green), $488 \mathrm{~nm}$ (blue) and $476.5 \mathrm{~nm}$ (violet). These three beams are again split into two beams to determine flow reversal using down-mixing. At low velocity, down-mixing helps in increasing overall accuracy of measured velocity. This additional shifting in frequency is performed in signal processing unit. The three shifted and three un-shifted beams from the multicolor beam generator are coupled with six fiber-optic couplers connected to a junction box. From junction box, these six beams are transported to the probe through an optical fiber cable. In 5-beam probe, all six beams (shifted and un-shifted beams of three colors) are coming out from five points through a single achromatic lens shown in Figure 3.13 (b).

\subsubsection{Laser induced fluorescence}

The LIF technique has a profound impact on experimental fluid dynamics research as it facilitates sheet illumination for the flow visualization. The sheet illumination and imaging technique in fluid dynamics give an insight visualization of fluid flow on the measurement plane. A typical LIF visualization system consists four main components such as laser source, optical lenses to convert laser beam into a sheet, fluorescence dye and imageacquiring device. The experimental set-up used for LIF is shown in Figure 3.14. 


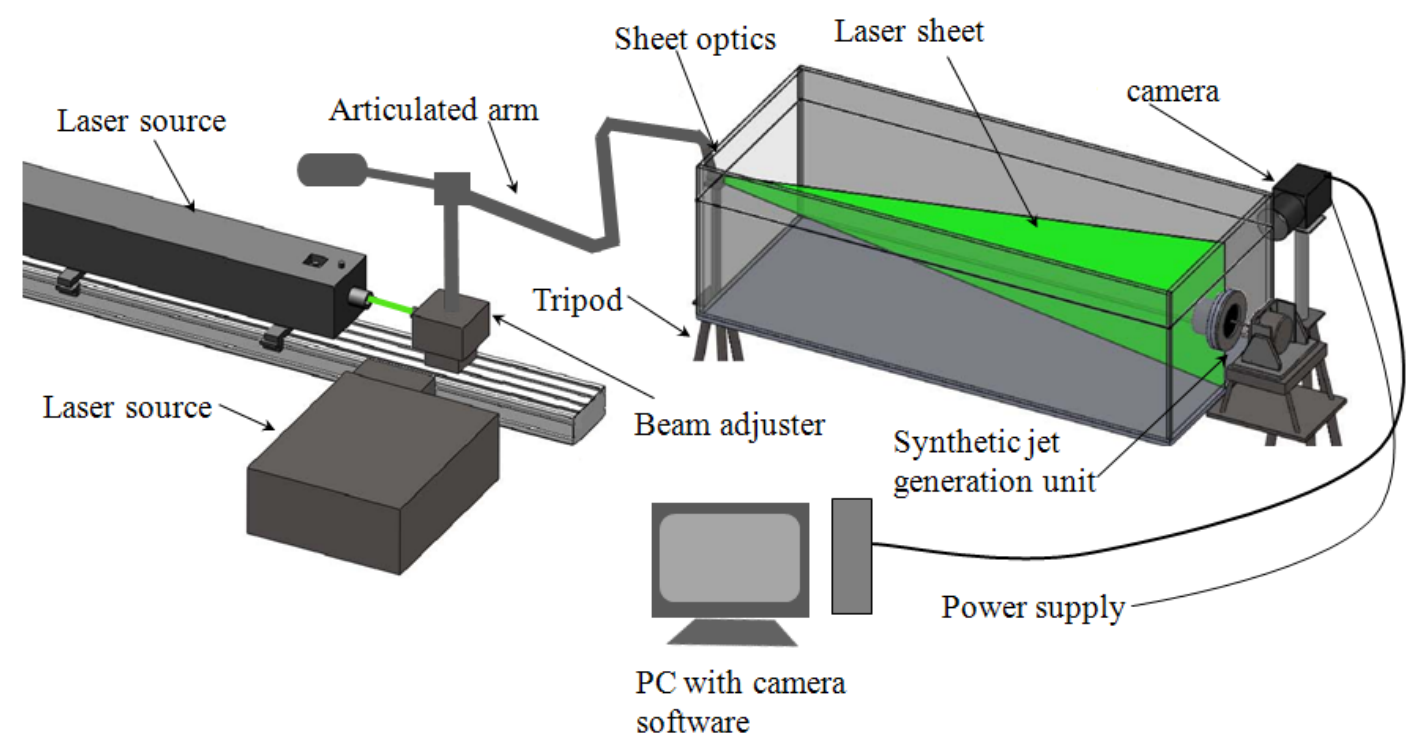

Figure 3.14: Schematic of experimental set-up used for laser induced fluorescence technique.

The laser source is a COHERENT ${ }^{\circledR}$ INNOVA-90, 4W continuous wave Argon-Ion Laser. For converting the laser beam into a thin sheet, initially, glass rods of different diameters are used. However, it cannot be used with ease because of its limited portability and difficulty in positioning. To circumvent this problem, an articulated arm is used to transport the laser-beam to the desired location for converting the beam into a thin sheet. The outlet end of articulated arm is fitted with a plano-concave cylindrical lens of focal length $(15 \mathrm{~mm})$ for generating laser sheet of $1.5 \mathrm{~mm}$ thickness. The whole sheet generation unit attached to the articulated arm is mounted on a tripod. The height and orientation of laser sheet is adjusted by tripod settings. The LIF images are captured with a resolution of $1280 \times 1280$ and a frame rate between 10 to 30 fps using Basler ${ }^{\circledR}$ A501-KC, CMOS camera having maximum frame rate of 74 fps. The frame rate of the camera is set based on the actuation frequency of synthetic jet, laser sheet intensity, and various camera parameters such as gain, exposer time etc.

The quality of LIF imaging strongly depends on the dye used during measurements. The most common aqueous LIF dyes are Fluorescein, Rhodamine-6G and Rhodamine-B. Among them Fluorescein sodium $\left[\mathrm{C}_{20} \mathrm{H}_{10} \mathrm{Na}_{2} \mathrm{O}_{5}\right]$ has more than $80 \%$ of peak energy 
absorption between wavelengths 460 to $520 \mathrm{~nm}$ with peak at $490 \mathrm{~nm}$. The emission wavelength band is higher than $20 \%$ of peak lies between the wavelength 510 to $520 \mathrm{~nm}$ with peak at $515 \mathrm{~nm}$. The quantum efficiency of the camera sensor lies between 400 to 700 $\mathrm{nm}$. The laser source used in multiline mode has excitation wavelengths of $514.5 \mathrm{~nm}, 488$ $\mathrm{nm}$ and $476.5 \mathrm{~nm}$. Hence, fluorescein sodium is chosen as the dye for LIF imaging. However, the disadvantage of using Fluorescein is that it has higher density $(1.602 \mathrm{gm} / \mathrm{cc})$ than that of Rhodamine (1.26 gm/cc). Fluorescein mixed with water (1:100), the resulting solution is found to be quite suitable for the LIF study. As mentioned earlier, three holes on the circumference of synthetic jet cavity are provided for the dye injection. During the LIF experiments, the water tank/tunnel is drained out and refilled periodically to avoid higher dye concentration in the water, which affects quality imaging.

\subsubsection{Bulk flow visualization}

The experimental set-ups for bulk flow visualization are similar to set-up for LIF imaging technique as shown in Figure 3.14 except the light source and dye used. The light source used for bulk flow visualization is a combination of 3-4 incandescent bulbs (60W) situated at different locations. The bulbs are covered with semitransparent papers to have diffused light over the imaging area. The proper positioning of bulbs are required as reflection from the glass walls of test section and shining surface of model affects the image quality. Different orientations and positioning of the bulbs are tried to capture the image with minimum reflection. The colored ink is used as the dye for bulk flow visualization. All the acquired images are processed by image processing tools to enhance the quality of images without affecting the flow features.

In experiments, various factors influence the final measured of data. The uncertainty analysis of various quantities has been carried out and the details of which is given in appendix-B. Uncertainty in the measurement of velocity using hot-film and LDV are equal 
to $\pm 4.1 \%$ and $\pm 3.7 \%$ respectively. Both the uncertainty in the actuation frequency of electromagnetic actuated synthetic jet (quiescent flow set-up) and in cam actuated synthetic jet (torpedo shape model) is found to be $\pm 5 \%$. The uncertainty in the Reynolds and Strouhal number are equal to $\pm 9.4 \%$ and $\pm 8 \%$ for quiescent flow set-up and $\pm 5.2 \% \pm 0.5 \%$ for torpedo shape model. $\mathrm{A} \pm 8 \%$ of uncertainty is obtained in the measurement of the velocity ratio. The error in vortex core identification is found to be $6.2 \%$ for 1 and $2 \mathrm{~Hz}$ actuation frequency and $12.6 \%$ for 4 and $6 \mathrm{~Hz}$ actuation frequency.

\subsection{Closure}

In this chapter, various experimental set-ups and measurement techniques used in the present study have been discussed. One set-up consisting of synthetic jet generator on the sidewall of a water tank is used to study the characteristics of circular and rectangular synthetic jets in quiescent flow. The other set-up consists of a torpedo shape model with built-in synthetic jet generator to study the synthetic jet in crossflow condition. The arrangements for hot-film measurement, LDV measurement, and flow visualization are also discussed. The hot-film set-up is only used in quiescent flow due to various constraints imposed by the quality of water. LDV is used for velocity measurement of synthetic jets in quiescent and in crossflow conditions. LIF imaging is also performed for both the abovementioned flow configurations. The detail of bulk flow visualization using the incandescent light used in crossflow experimental set-up is also presented. 


\section{Chapter 4}

\section{Synthetic jet in quiescent flow: effect of orifice shape and excitation frequency}

The characteristics of synthetic jet depend on geometrical parameters such as orifice curvature, thickness to orifice diameter ratio, in-line or off-line diaphragm displacement and geometry of the cavity. However, the orifice curvatures have a significant impact on the evolution of synthetic jet or on the interaction of synthetic jet with the surroundings. The shape of the orifice governs convection, entrainment of synthetic jet flow with surroundings, spreading and mixing, thrust and many more aspects of synthetic jet. Two widely used orifice shapes are: (a) circular or axi-symmetric shaped orifice that generates vortex ring of constant curvature and (b) non-circular orifices such as rectangular, elliptical and triangular generating vortex ring of varying vortex tube curvature.

The non-circular vortex ring has different behaviour compared to the circular vortex ring. Bio-Sevart's law gives the induced velocity of the vortex tube element . The induced velocity of vortex tube element ' $\mathrm{dA}$ ' as shown in Figure 4.1 is expressed as:

$$
V_{\text {induced }}=\frac{\Gamma}{4 \pi a} \log \left(\frac{a}{r}\right)
$$




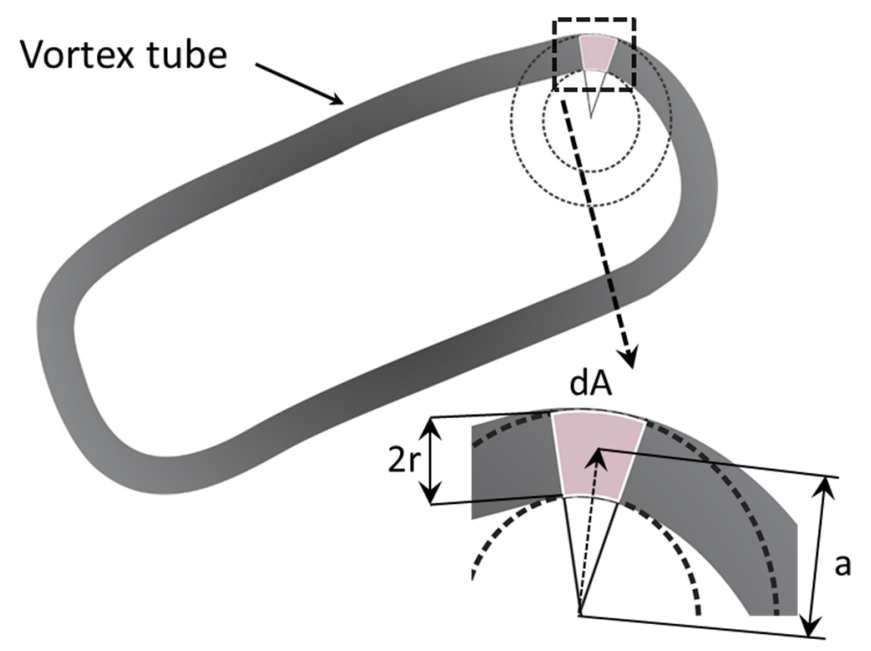

Figure 4.1: Schematic representation of induced velocity of non-circular vortex tube.

Where $\Gamma$ is circulation of vortex ring, a is ring radius, $r$ is radius of vortex tube and $V_{\text {induced }}$ is the induced velocity of vortex tube element. For a rectangular shaped vortex tube, the curvature is higher at the corners and lower or negligible at the center. Considering a small segment of vortex tube dA of an imaginary vortex ring of constant circulation and radius, the induced velocity depends on the vortex radius and hence the term $\ln (\mathrm{a} / \mathrm{r}) / \mathrm{a}$ in equation 4.1 increases as 'a' decreases and vice versa. Therefore, the segment of vortex tube having larger curvature i.e. smaller radius of imaginary vortex ring has a higher induced velocity than the other section of vortex tube having comparatively larger radius. The corners of vortex tube that have the highest induced velocity stretch the remaining portion of vortex tube. For a rectangular shaped orifice, the induced velocity of different segment in decreasing order are; corners, shorter side and the larger side. Hence, the characteristics of non-circular rings are significantly different from the circular one. Therefore, the focus of this chapter is to study the behaviour of circular and rectangular shape orifice at different actuation frequencies of diaphragm. It is to be noted that for simplicity, the frequencies are given its nearest integer values though the actual values are fractional while discussing results. 
An experimental set-up consisting of a synthetic jet generator on the sidewall of a water tank has been used to study the characteristics of circular and rectangular orifice synthetic jet in quiescent flow condition. LIF imaging in two orthogonal planes has been carried out for visualization of streamwise and cross-stream vortex structure. Mean velocity and velocity fluctuation measurements have been carried out using hot-film anemometry.

The experimental results have been discussed in four sections: (a) experimental conditions, (b) circular orifice synthetic jet, (c) rectangular orifice synthetic jet and (d) timefrequency analysis. Circular orifices having diameter of 8, 10 and $13 \mathrm{~mm}$ are used and the actuation frequency is set equal to 1,2, 4 and $6 \mathrm{~Hz}$. The aspect ratio of the rectangular orifice is set equal to 1,2 and 4 with hydraulic diameter equal to $12 \mathrm{~mm}$. The actuation frequencies are kept same as that of circular orifices. In the last section, the velocity signal is analysed in time domain, in frequency domain and in joint time-frequency domain for a circular, square and two rectangular orifices of aspect ratio two and four actuating at 1, 6 and $18 \mathrm{~Hz}$ frequency.

\subsection{Experimental conditions}

The synthetic jet mounted on the sidewall of water tank is actuated using electromagnetic actuator and frequency controller, where the diaphragm displacement is coupled with actuation frequency. Since the diaphragm displacement at a particular frequency is constant for the present set-up, independent control of amplitude is not possible. The volume of fluid expelled from the orifice cavity during forward stroke is a function of frequency plus the diaphragm displacement. However, the actuation frequency is considered as the parameter for discussion in place of fluid volume displaced or ejected out from the orifice. The corresponding non-dimensional numbers i.e. Reynolds number $(\mathrm{Re})$ and Strouhal number $\left(\mathrm{S}_{\mathrm{t}}\right)$ are reported in Table 1-3. The calculations of non-dimensional numbers are based on the average velocity of synthetic jet during the ejection stroke only (details have been 
discussed in Chapter 3). Nomenclature used for reporting results of circular and noncircular orifices are shown in Figure 4.2. LIF imaging at 30 frame per second is used for flow visualization of synthetic jet. Considering the flow symmetry for both circular and square orifice, images are acquired at only one plane (XZ-plane). For rectangular orifice of aspect ratio two and four, the images are acquired in two orthogonal planes (XY and XZ) as shown in Figure 4.2 (b).



(a) Circular orifice



Circular orifice

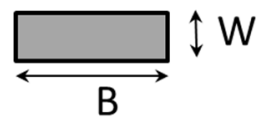

Non-circular orifice

(c) Orifice notation

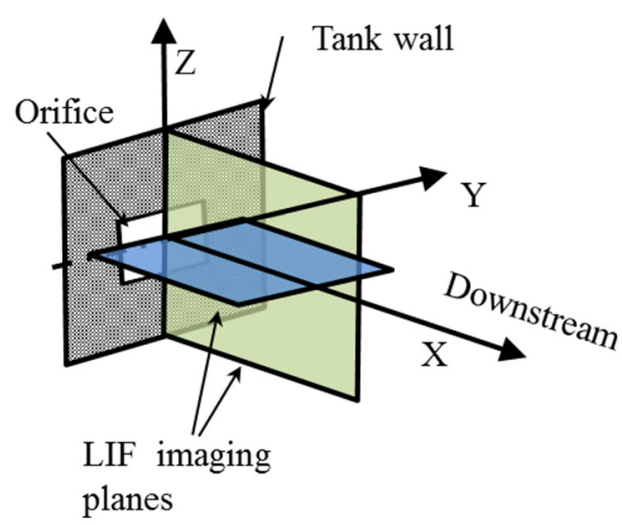

(b) Rectangular orifice

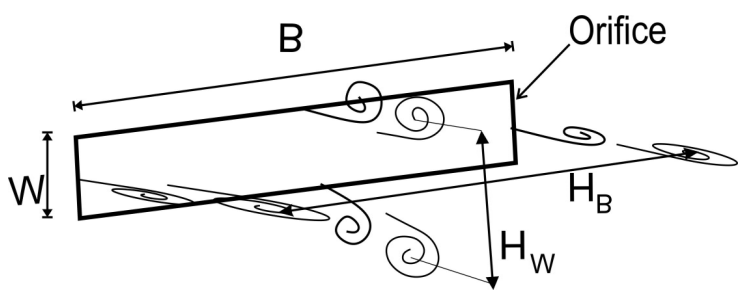

(d) Separation distance

Figure 4.2: Nomenclature for reporting the experimental results: (a) circular orifice flow visualization plane, (b) rectangular orifice flow visualization planes, (c) orifice notations and (d) separation distance between the counter rotating vortices in plane $\mathrm{XZ}$ and $\mathrm{XY}$. 
Table 4.1: Geometrical and experimental condition details for experiment with circular orifice

\begin{tabular}{|c|c|c|c|c|c|c|c|}
\hline \multirow[t]{2}{*}{ Orifice } & \multirow[t]{2}{*}{ Shape } & \multirow{2}{*}{$\begin{array}{l}\text { Size } \\
(\mathrm{mm})\end{array}$} & \multicolumn{2}{|c|}{$\begin{array}{l}\text { Actuation frequency } \\
\text { (f) } \mathrm{Hz}\end{array}$} & \multirow{2}{*}{$\begin{array}{l}\text { Reynolds } \\
\text { Number } \\
\text { (Re) }\end{array}$} & \multirow[t]{2}{*}{$\begin{array}{l}\text { Strouhal } \\
\text { Number }\left(S_{t}\right)\end{array}$} & \multirow[t]{2}{*}{ L/D } \\
\hline & & & Actual & Round-off & & & \\
\hline \multirow{4}{*}{ D8 } & \multirow{4}{*}{ Circular } & \multirow{4}{*}{8} & 1.02 & 1 & 506 & 0.14 & 7.1 \\
\hline & & & 1.60 & 2 & 1462 & 0.10 & 10.0 \\
\hline & & & 3.65 & 4 & 6933 & 0.07 & 14.2 \\
\hline & & & 5.88 & 6 & 9533 & 0.05 & 20.0 \\
\hline \multirow{4}{*}{ D10 } & \multirow{4}{*}{ Circular } & \multirow{4}{*}{10} & 1.02 & 1 & 462 & 0.28 & 3.6 \\
\hline & & & 1.60 & 2 & 1056 & 0.20 & 5.0 \\
\hline & & & 3.65 & 4 & 3328 & 0.14 & 7.2 \\
\hline & & & 5.88 & 6 & 7380 & 0.10 & 10.0 \\
\hline \multirow{4}{*}{ D13 } & \multirow{4}{*}{ Circular } & \multirow{4}{*}{13} & 1.02 & 1 & 370 & 0.60 & 1.7 \\
\hline & & & 1.60 & 2 & 845 & 0.43 & 2.3 \\
\hline & & & 3.65 & 4 & 2663 & 0.30 & 3.3 \\
\hline & & & 5.88 & 6 & 5904 & 0.22 & 4.5 \\
\hline
\end{tabular}

(* For simplicity in notation and discussion the actuation frequency are rounded to their nearest integer, however all the calculations are based on the exact value)

Table 4.2: Geometrical and experimental conditions details for experiment with rectangular orifice

\begin{tabular}{|c|c|c|c|c|c|c|c|}
\hline \multirow[t]{2}{*}{ Orifice } & \multirow[t]{2}{*}{ Shape } & \multirow{2}{*}{$\begin{array}{l}\text { Size } \\
(\mathrm{mm})\end{array}$} & \multicolumn{2}{|c|}{$\begin{array}{l}\text { Actuation } \\
\text { frequency (f) } \mathrm{Hz}\end{array}$} & \multirow{2}{*}{$\begin{array}{l}\text { Reynolds } \\
\text { Number } \\
\text { (Re) }\end{array}$} & \multirow{2}{*}{$\begin{array}{l}\text { Strouhal } \\
\text { Number } \\
\left(S_{t}\right)\end{array}$} & \multirow[t]{2}{*}{$L / D_{h}$} \\
\hline & & & Actual & Round-off & & & \\
\hline \multirow{4}{*}{ AR1 } & \multirow{4}{*}{ Square } & \multirow{4}{*}{$12 \times 12$} & 1.02 & 1 & 242 & 0.61 & 1.6 \\
\hline & & & 1.60 & 2 & 553 & 0.43 & 2.3 \\
\hline & & & 3.65 & 4 & 1742 & 0.30 & 3.3 \\
\hline & & & 5.88 & 6 & 3864 & 0.22 & 4.5 \\
\hline \multirow{4}{*}{ AR2 } & \multirow{4}{*}{ Rectangular } & \multirow{4}{*}{$9 \times 18$} & 1.02 & 1 & 215 & 0.68 & 1.5 \\
\hline & & & 1.60 & 2 & 491 & 0.48 & 2.1 \\
\hline & & & 3.65 & 4 & 1549 & 0.34 & 2.9 \\
\hline & & & 5.88 & 6 & 3435 & 0.25 & 4.0 \\
\hline \multirow{4}{*}{ AR4 } & \multirow{4}{*}{ Rectangular } & \multirow{4}{*}{$7.5 \times 30$} & 1.02 & 1 & 155 & 0.95 & 1.1 \\
\hline & & & 1.60 & 2 & 354 & 0.67 & 1.5 \\
\hline & & & 3.65 & 4 & 1115 & 0.47 & 2.1 \\
\hline & & & 5.88 & 6 & 2473 & 0.34 & 2.9 \\
\hline
\end{tabular}

(* For simplicity in notation and discussion the actuation frequency are rounded to their nearest integer, however all the calculations are based on the exact value) 
Table 4.3: Geometrical and experimental condition details for experiment on spectral analysis

\begin{tabular}{|c|c|c|c|c|c|c|c|}
\hline \multirow[t]{2}{*}{ Orifice } & \multirow[t]{2}{*}{ Shape } & \multirow[t]{2}{*}{$\begin{array}{l}\text { Size } \\
(\mathrm{mm})\end{array}$} & \multicolumn{2}{|c|}{$\begin{array}{l}\text { Actuation frequency } \\
\text { (f) } \mathrm{Hz}\end{array}$} & \multirow{2}{*}{$\begin{array}{l}\text { Reynolds } \\
\text { Number } \\
\text { (Re) }\end{array}$} & \multirow{2}{*}{$\begin{array}{l}\text { Strouhal } \\
\text { Number } \\
\left(\mathbf{S}_{\mathrm{t}}\right)\end{array}$} & \multirow[t]{2}{*}{$\mathbf{L} / \mathbf{D}_{\mathbf{h}}$} \\
\hline & & & Actual & Round-off & & & \\
\hline \multirow{3}{*}{$\mathrm{D} 10$} & \multirow{3}{*}{ Circular } & \multirow{3}{*}{10} & 1.02 & 1 & 462 & 0.28 & 3.6 \\
\hline & & & 5.88 & 6 & 7380 & 0.10 & 10.0 \\
\hline & & & 17.8 & 18 & 20374 & 0.10 & 10.18 \\
\hline \multirow{3}{*}{ AR1 } & \multirow{3}{*}{ Square } & \multirow{3}{*}{$12 \times 12$} & 1.02 & 1 & 242 & 0.61 & 1.6 \\
\hline & & & 5.88 & 6 & 3864 & 0.22 & 4.5 \\
\hline & & & 17.8 & 18 & 13333 & 0.22 & 4.63 \\
\hline \multirow{3}{*}{ AR2 } & \multirow{3}{*}{ Rectangular } & \multirow{3}{*}{$9 \times 18$} & 1.02 & 1 & 215 & 0.67 & 1.5 \\
\hline & & & 5.88 & 6 & 3435 & 0.24 & 4.0 \\
\hline & & & 17.8 & 18 & 11852 & 0.25 & 4.12 \\
\hline \multirow{3}{*}{ AR4 } & \multirow{3}{*}{ Rectangular } & \multirow{3}{*}{$7.5 \times 30$} & 1.02 & 1 & 155 & 0.91 & 1.1 \\
\hline & & & 5.88 & 6 & 2473 & 0.34 & 2.9 \\
\hline & & & 17.8 & 18 & 8533 & 0.34 & 2.96 \\
\hline
\end{tabular}

(* For simplicity in notation and discussion the actuation frequency are rounded to their nearest integer, however all the calculations are based on the exact value)

Figure 4.2 (c) shows the notation of circular and non-circular orifice geometry. Нв and $\mathrm{Hw}$ are the sizes of vortex ring measured in LIF images in XY-plane parallel to breadth (B) and in XZ-plane parallel to width (W) respectively (see Figure 4.2 (d)). For circular orifice, the size of vortex ring is denoted by $\mathrm{H}_{\mathrm{D}}$. The velocity measurement is carried out by S-type hot-film. The data acquisition rate is $500 \mathrm{samples} / \mathrm{sec}$ for circular orifices and $1000 \mathrm{samples} / \mathrm{sec}$ is for non-circular orifices. The three non-dimensional numbers, which govern the characteristics of synthetic jet in quiescent flow are Reynolds number, Strouhal number and formation number (details have been discussed in appendix A). The formation number $(\mathrm{L} / \mathrm{D})$, Reynolds number $(\mathrm{Re})$ and Strouhal number $\left(\mathrm{S}_{\mathrm{t}}\right)$ based on slug length $(\mathrm{L})$ and velocity $\left(\mathrm{U}_{0}\right)$ for circular orifices, rectangular orifices and spectral analysis cases are tabulated in Table 4.1, Table 4.2 and Table 4.3 respectively. 


\subsection{Synthetic jets with circular orifice}

The vortex ring of synthetic jet is affected by the flow field developed nearer to the jet exit. If the newly formed vortex ring does not have enough velocity to escape from the suction zone, there will be partial suction of ejected fluid back to the cavity. Therefore, the fluid at the orifice exit may oscillate at some actuation frequency and diaphragm displacement. The escape velocity of synthetic jet depends on the strength of feeding shear layer to the forming vortex ring at the orifice exit. The orifice diameter and actuation frequency coupled with diaphragm displacement are selected in such a way that the backward stroke of diaphragm is unable to digest back the ejected fluid. The formation of vortex ring in synthetic jet depends on various parameters such as actuation frequency, amplitude of oscillation, shape, thickness of orifice and cavity geometry. The normalized stroke length L/D ratio represents the combined effect of these parameters. Behaviour of circular synthetic jet is presented below in the following sequence: (a) flow visualization (b) vortex size and (c) timeaveraged velocity field.

\subsubsection{Flow visualization}

Laser Induced Fluorescence visualization (LIF) has been used to study the structure and the evolution of vortex ring at different stages of the synthetic jet. In the range of Reynolds number (Re) 370 to 9553 and Formation number (L/D) 1.6 to 20, the flow structure seen in Figure 4.3 and Figure 4.4 matches with that of Gharib et al. (1998) even though the experimental conditions like orifice to thickness ratio, velocity program of diaphragm, water depth etc. are different in both the studies. Gharib et al. (1998) reported the formation of circular vortex ring generated using piston-cylinder actuation mechanism for circular nozzle in the range of $\mathrm{L} / \mathrm{D}=2$ to 14.5 and Reynolds number 600 to 2300 , where the Reynolds number and L/D ratio are based on the running mean of piston velocity during 
forward stroke. The pattern of synthetic jet flow structures reported by Crook \& Wood (2001) for circular orifice in the range of $\mathrm{L} / \mathrm{D}=2$ to 10 and $\mathrm{Re}=330$ to 2300 (based on averaged exit velocity), which are also similar to the captured LIF images shown in this study.

The flow visualization images in XZ-plane for 8,10 , and $13 \mathrm{~mm}$ orifices are shown in Figure 4.3 ( 1 and $2 \mathrm{~Hz}$ actuation frequency) and in Figure 4.4 (4 and $6 \mathrm{~Hz}$ actuation frequency). The amount of fluid displaced by the diaphragm, which is also equal to the fluid expelled out through orifice during forward stroke are 2854, 4021, 5710 and 8041 $\mathrm{mm}^{3}$ at $1,2,4$, and $6 \mathrm{~Hz}$ actuation frequency respectively.

In Figure 4.3 (a) and (b) for 8 and $10 \mathrm{~mm}$ circular orifice, leading vortex rings are well separated and laminar at $1 \mathrm{~Hz}$ actuation frequency. However, at same actuation frequency and fluid expulsion, an increase in diameter from 8 to $10 \mathrm{~mm}(57 \%$ increase in cross sectional area) leads to reduction in L/D ratio from 7.2 to 3.1 with a comparative slow reduction in Reynolds number from 510 to 462. No significant change in flow structure is observed between $8 \mathrm{~mm}$ and $10 \mathrm{~mm}$ orifice, except a tiny trailing jet behind the leading vortex ring of $8 \mathrm{~mm}$ orifice. When the diameter is further enlarged from 10 to $13 \mathrm{~mm}(69 \%$ increase in cross sectional area), $\mathrm{L} / \mathrm{D}$ ratio reduces to 1.64 and Reynolds number reduces to 370 (see Figure 4.3 (c)). The complete rollup of leading vortex ring is seized at this condition. The fluid expelled out from the orifice has sufficient velocity, which allow the shear layer to rollup but got restricted by following suction stroke. Therefore, due to insufficient self-induced velocity, the vortex ring is not able to travel far enough from the orifice plate before beginning of the next suction stroke. The shear layers close to the orifice sides ingested back into the orifice cavity. This leads to formation of heart shaped flow structure near the orifice exit. The vortex rings are also closely spaced and not distinct. Similar observation is also reported by Watson et al. (2003) through experiments on a 
circular synthetic jet of $5 \mathrm{~mm}$ diameter with diameter to thickness ratio equal to one at 50 $\mathrm{Hz}$ actuation frequency using electromagnetic-diaphragm actuation mechanism in air.

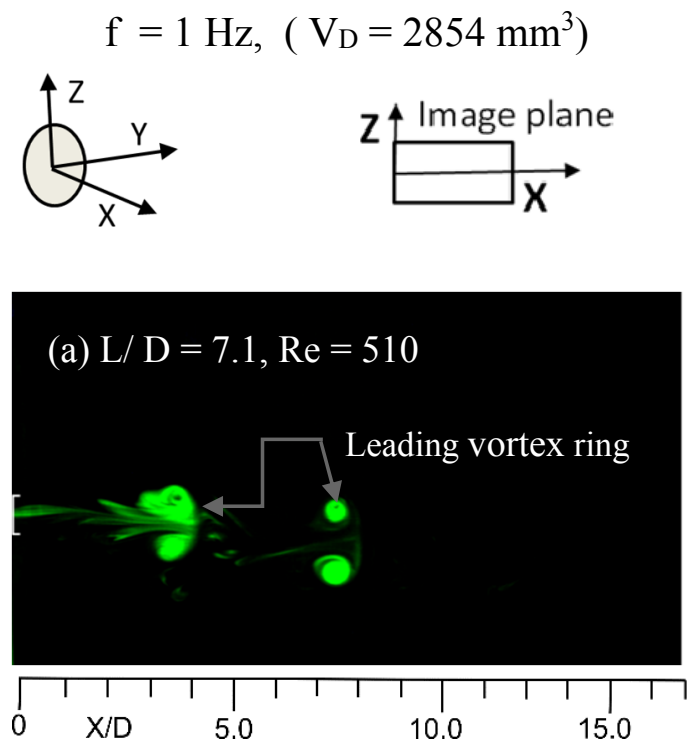

$$
\begin{aligned}
& \mathrm{f}=2 \mathrm{~Hz},\left(\mathrm{~V}_{\mathrm{D}}=4021 \mathrm{~mm}^{3}\right) \\
& \underset{\mathrm{X}}{\mathrm{Z}}
\end{aligned}
$$

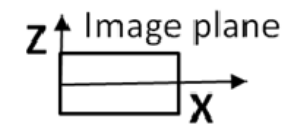

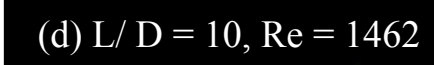

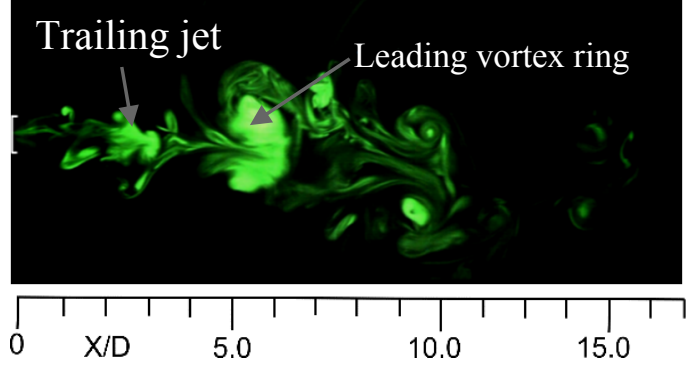

$\underset{\infty}{ }$
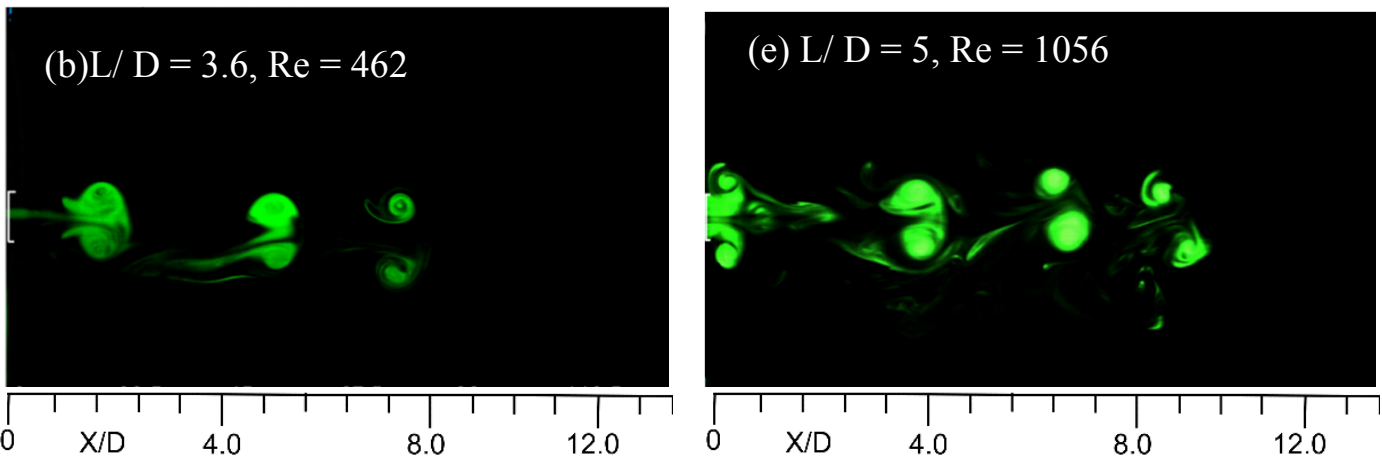

릉


$\frac{\theta}{\omega}$

Figure 4.3: Flow visualization images of synthetic jet in XZ-plane (side view) for $8 \mathrm{~mm}$ (D8), $10 \mathrm{~mm}$ (D10), and $13 \mathrm{~mm}$ (D13) circular shaped orifices at 1 and $2 \mathrm{~Hz}$ actuation frequency. 




$$
\begin{aligned}
& \mathrm{f}=6 \mathrm{~Hz},\left(\mathrm{~V}_{\mathrm{D}}=8041 \mathrm{~mm}^{3}\right) \\
& \stackrel{\mathrm{X}}{\mathrm{Z}}
\end{aligned}
$$




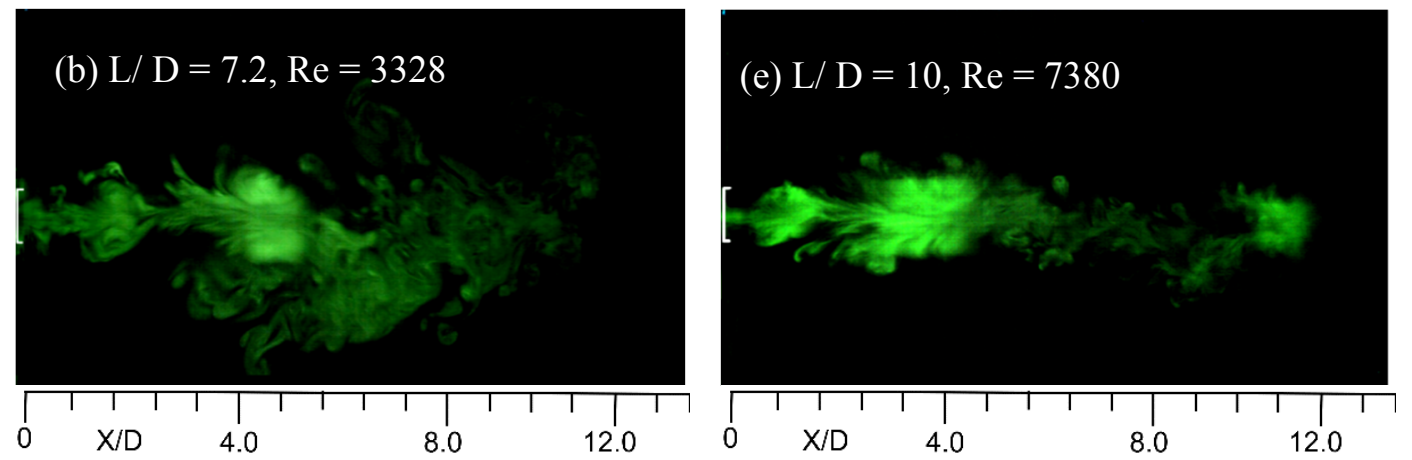

$\frac{\theta}{\ominus}$
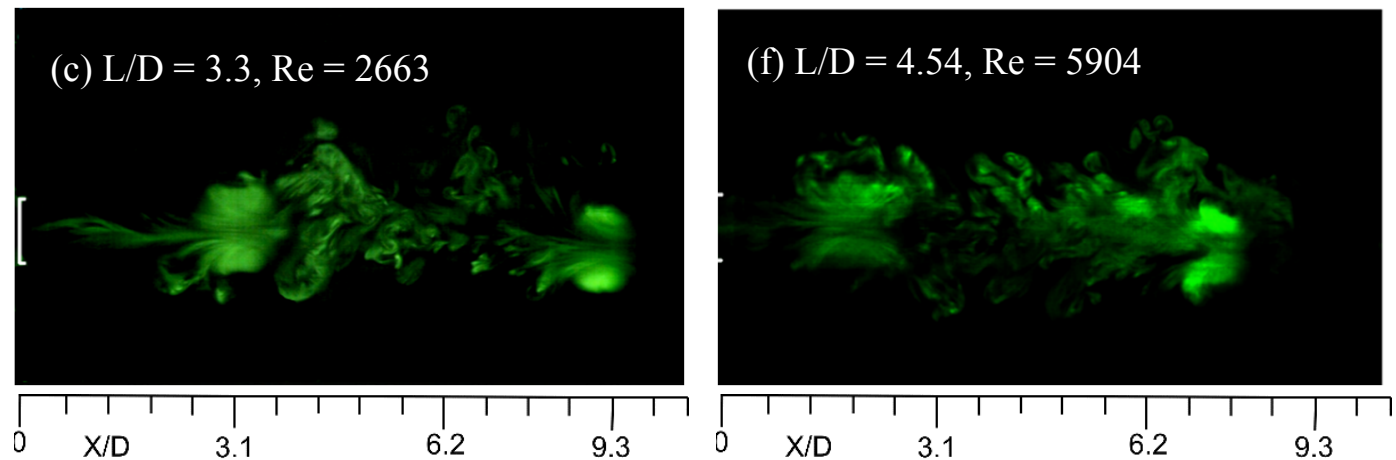

$\underset{\omega}{\operatorname{co}}$

Figure 4.4: Flow visualization images of synthetic jet in XZ-plane (side view) for $8 \mathrm{~mm}$ (D8), $10 \mathrm{~mm}$ (D10), and $13 \mathrm{~mm}$ (D13) circular shaped orifices at 4 and $6 \mathrm{~Hz}$ actuation frequency.

The synthetic jet sheds an isolated and clearly distinguishable trailing jet behind the leading vortex ring for $2 \mathrm{~Hz}$ actuation frequency as shown in Figure 4.3 (d) for $8 \mathrm{~mm}$ orifice at $\mathrm{L} / \mathrm{D}=10$ and $\mathrm{Re}=1462$. It was observed experimentally by Gharib et al. (1998) and analytically by Mohseni and Gharib (1998) that the limiting value of L/D occurs when the generating apparatus is unable to supply the energy, circulation and impulse at a rate 
comparable with the requirement that a steadily translating vortex ring needs. Once the L/D ratio exceeds the limiting value, the leading vortex ring refuses to accommodate the complete volume of ejected fluid and sheds the extra fluid behind the leading vortex ring. As soon as the vorticity in the shear layer ceases to flow into the vortex core of leading vortex ring, pinch-off process starts, and the leading vortex ring gets detached from supply of fluid. The left over fluid behind the leading vortex ring is termed as trailing jet. The circulation, energy of trailing jet is comparatively less than that of the leading vortex ring. Three different forms of trailing jet are observed at $2 \mathrm{~Hz}$ actuation frequency in Figure 4.3 (d-f), conical and jet like trailing jet for $8 \mathrm{~mm}$ orifice, trailing jet in the form of coherent structure for $10 \mathrm{~mm}$ orifice and sparsely distributed small scale vortices behind the leading vortex ring for $13 \mathrm{~mm}$ orifice. The ingestion of trailing jet by leading vortex ring of the following cycle is observed in the sequence of images for 8 and $10 \mathrm{~mm}$ orifices at $2 \mathrm{~Hz}$ actuation frequency.

Figure 4.4 (a) and (b) shows the visualization images for further increases of actuation frequency to 4 and $6 \mathrm{~Hz}$. The trailing jet becomes stronger, more chaotic and interlinked or closely spaced to the leading vortex rings with increase in actuation frequency for all orifice sizes. The decrease in inter spacing between the adjacent vortex rings with increase in Reynolds number and decrease in Strouhal number multiply the mutual interaction between the vortex rings. This is also attributed to increased chaos/turbulence, which promotes the early vortex breakup and mixing. A vortex ring has a vortex core surrounded by layers or mixture of ejected and entrained surrounding fluid (see Figure 4.5(a)). The core is a closed tube having equal area and circulation and a simple perturbation in core has a strong impact on the behaviour of vortex ring. Sullivan et al. (2008) reported that there is an azimuthal instability associated with the core of vortex tube known as crow instability. This instability has waves over the core of vortex tube that grows 
and takes energy from the ring and slows down the self-induced velocity. A loss in velocity is compensated by the growth in the size of vortex core. Due to subsequent diffusion of vorticity, vortex rings loses its strength and finally disappears.

Figure 4.4 shows a drift of vortex ring from the synthetic jet centerline at 4 and 6 $\mathrm{Hz}$ actuation frequency contrary to lower actuation frequencies of 1 and $2 \mathrm{~Hz}$ shown in Figure 4.3. In cases where the synthetic jet have leading vortex ring or have weaker trailing jet, the drifting of vortex ring is less and the vortex ring propagates in the near vicinity of synthetic jet centerline. However, an increase in Reynolds number and/or actuation frequency increases the tendency of vortex ring drifting away from the synthetic jet centerline. The advantage is that the drifting of leading vortex ring or trailing jet from the synthetic jet centerline increases the overall spreading of synthetic jet. The probable reason may be linked with the inherent instability in vortex core, which generates a wave over the vortex core known as Kelvin waves. Unlike the counter rotating vortices, a vortex ring velocity significantly depends on its curvature and core vorticity. As the vortex ring convects under its self-induced velocity in direction normal to the plane of the ring, the core undergoes different stages of diffusion and distortion. The inter-connection of Kelvin waves with diffusion and distortion of coherent structure present in the surrounding affects the vortex ring propagation in downstream direction. The successive rings from the same ring generator i.e. the synthetic jet at different time instants produce the Kelvin waves of different phase and amplitude. The Kelvin wave interaction is a complex process due to time and space dependency and early prediction is difficult. Higher induced velocity with smaller vortex ring diameter is more prone to Kelvin waves of larger amplitude (Sullivan et al., 2008) and this may be one of the reasons for larger drifting of vortex ring from the centerline at 4 and $6 \mathrm{~Hz}$ actuation frequency. Apart from the diffusion and distortion of vortex core, different phenomena i.e. mixing of ejected fluid with the surroundings, 
turbulence in surroundings, and wake behind the vortex ring, entrainment and detrainment of fluid from the vortex rings also affects the vortex ring behaviour and its propagation.

Zaroodny (1966) observed that as the vortex ring propagates, a fresh mass of surroundings fluid is always available for mixing, thus mixing has stronger influence on the growth of vortex ring than the diffusion or any other factors. The growth of vortex ring is an important criterion to characterize the behaviour of synthetic jet. The separation distance between the counter rotating vortices in LIF images provides insight about the vortex ring development at different downstream locations. The separation distance (HD) between the cores of counter rotating vortices (which is basically a cross-sectional view of a vortex ring) of vortex ring measured from the sequence of LIF images (see in Figure 4.5 (a)) can provide information about the effect of orifice diameter and actuation frequency on the size of the vortex ring. It may be noted that the assumption of maximum concentration of dye at the center of core may be a source of error for this measurement. Even though the measured distances are not at exact location of core, it can give an overview of vortex growth and its sustainability in downstream direction. The separation distance between the cores of counter rotating vortices of circular vortex ring is proportional to the vortex size (Sullivan et al., 2008). Therefore, it can be assumed that the separation distance between the cores of counter rotating vortices in LIF images can be used as the representative of vortex size variation. The separation distances $H_{D}$ in downstream direction between the vortex cores are shown in Figure 4.5 (b), (c) and (d) for orifice sizes of 8,10 and $13 \mathrm{~mm}$ respectively. Separate curves fitting the loci on the locus of upper core in downstream direction is also shown for each frequency separately and all frequencies combined together.. The curve fit at each frequency represents the variation in the size of vortex ring at different actuation frequencies and orifice sizes as shown in Table 4.1. The curve fit on the cumulative data of all frequencies for a particular orifice helps in 
comparing the overall trend of vortex size variation within the considered frequency range for different sizes of orifice.

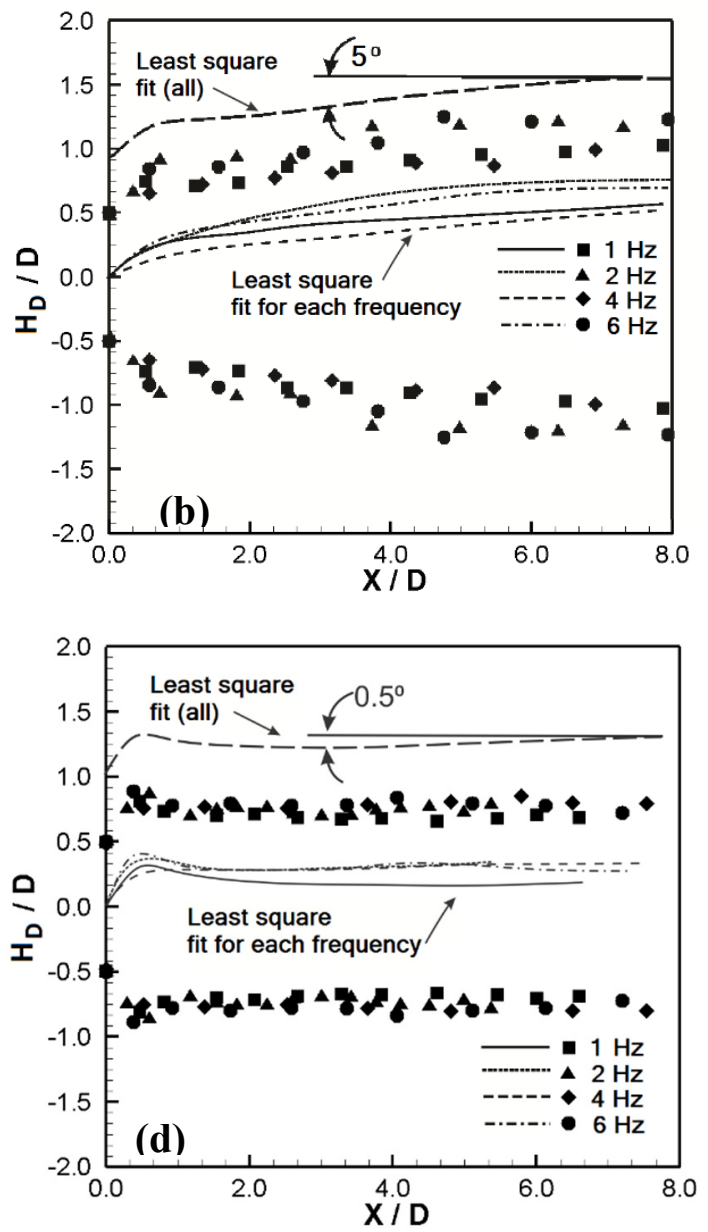

Figure 4.5: (a) Schematic of separation distance between the cores of counter rotating vortices in LIF images. The measured distance for (b) $8 \mathrm{~mm}$, (c) $10 \mathrm{~mm}$, and (d) $13 \mathrm{~mm}$ are shown at 1, 2, 4, and $6 \mathrm{~Hz}$ actuation frequency. ( $*$ The fitted curves are shifted up and down by a value 0.5 for clarity).

For $8 \mathrm{~mm}$ orifice in Figure 4.5 (b), the size of vortex core gradually increases in downstream direction. The corresponding fits according to individual frequency and cumulative data are shown in the figure. The overall vortex core separation distance for 13 mm orifice (see Figure 4.5 (d)) is less as compared to the other frequencies (see Figure 4.5(b) and (c)). The expansion of vortex ring or separation distance between counter rotating vortices increases in downstream direction for $8 \mathrm{~mm}$ orifice but remains almost same for 10 and $13 \mathrm{~mm}$ orifices till $\mathrm{X} / \mathrm{D}=6$. The trend of vortex growth in downstream direction changes as the orifice diameter increases from $8 \mathrm{~mm}$ to $13 \mathrm{~mm}$. After the 
overshoot of vortex ring $(\mathrm{X} / \mathrm{D}<3)$ at orifice exit, the slope of least square fit at all frequencies shown in Figure 4.5 decreases for increases in orifice diameter from 8 to 13 $\mathrm{mm}$. One possible reason for this drop may be the pinch-off process of leading vortex ring. Mohseni et al. (2001) reported that at pinch-off moment, the axial velocity of following shear layer is equal to the translation velocity of vortex ring. During vortex rollup, the shear layer is forced towards the axis of symmetry where it loses its strength. They also observed that pinch-off process can be delayed by moving the feeding shear layer away from the orifice center or by gradually increasing the speed of feeding shear layer during vortex roll up. Since increasing the orifice diameter from $8 \mathrm{~mm}$ to $13 \mathrm{~mm}$ at a particular frequency pushes the shear layer away from the synthetic jet centerline and hence delays the vortex pinch off. The maximum width of region containing the locus of upper or lower vortex cores at 1, 2, 4 and $6 \mathrm{~Hz}$ is approximately $0.5 \mathrm{D}$ for $8 \mathrm{~mm}, 0.25 \mathrm{D}$ for $10 \mathrm{~mm}$ and negligible for $13 \mathrm{~mm}$ orifice in the range of $\mathrm{X} / \mathrm{D}=0.5$ to 6 (Figure $4.5(\mathrm{~b}-\mathrm{d})$ ). The reason could be the azimuthal instability associated with the vortex core. Nitsche (2006) suggested that the vortex ring of smaller cross section are subjected to larger azimuthal instability, which is unstable and grow as the core size decreases. The other reason is that the velocity of vortex ring increases towards the vortex core and consequently the pressure decreases thus drawing more fluid inside the vortex ring from surroundings and increase its size. Since an increase in diameter of moving vortex ring reduces the velocity gradient inside the vortex ring (Sullivan et al. (2008), Nitsche (2006)), entrainment of surrounding fluid is also suppressed.

In Figure $4.5(\mathrm{~d})$, there is significant reduction in the vortex ring size before $\mathrm{X} / \mathrm{D}=$ 1. The vortex ring diameter increases until the feeding shear layer is consumed inside the forming vortex ring and the diameter of vortex ring reaches its maximum value prior to the end of expulsion stroke. Due to the suction stroke of the following cycle, fluid flow towards 
the cavity begins near the orifice sides, which pushes the feeding shear layer towards the orifices center and finally seizes the fluid flow inside the forming vortex ring. Further, the reversed flow towards the orifice cavity in suction stroke, squeezes the forming vortex ring at its rear end and the vortex ring shrinks. The shrinking of vortex ring after its detachment from the supply of feeding shear layer is termed as the overshoot of the vortex ring. Shuster and Smith (2007) concluded that the amplitude of overshoot is more prominent for synthetic jet having smaller value of formation number for a synthetic jet of a circular orifice having diameter to thickness ratio equal to 0.5 . A reduction of $50 \%, 20 \%$ and $10 \%$ in vortex ring diameter is reported by them at $\mathrm{L} / \mathrm{D}=1,2$ and 3 respectively. A $37 \%, 24 \%$ and $20 \%$ reduction in vortex ring is observed at $\mathrm{L} / \mathrm{D}=1.6,2.3$ and 3.3 respectively for 13 $\mathrm{mm}$ orifice. The vortex rings shrink about $8.5 \%$ and $13 \%$ for 8 and $10 \mathrm{~mm}$ orifice size respectively at $1 \mathrm{~Hz}$ actuation frequency. For other cases either there is no reduction in vortex ring diameter or they are negligible 


\subsubsection{Time-averaged centerline velocity}

Time-averaged velocity distribution along the synthetic jet centerline can be used to show different regions of synthetic jet evolution (see Figure 4.6) by observing the flow structures seen in Figure 4.3 and Figure 4.4. The velocity variation along the synthetic jet centerline can be broadly divided into four regions: (a) vortex rollup and suction region, (b) steadily translating vortex ring region, (c) vortex breakdown region, and (d) dissipation region of small-scale vortices.



Figure 4.6: Various regions of time-averaged velocity profile along the synthetic jet centerline.



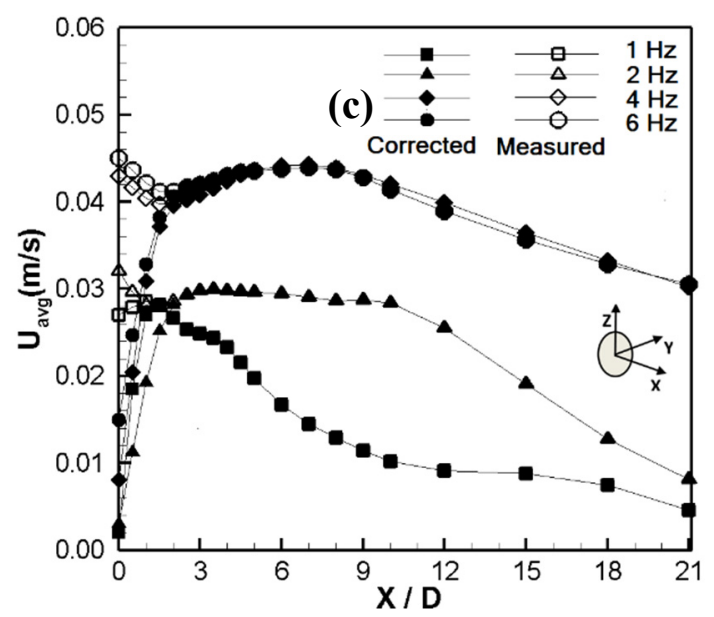

Figure 4.7: Time-averaged streamwise velocity measured along the synthetic jet centerline at different downstream locations actuating at 1, 2, 4 and $6 \mathrm{~Hz}$ for orifice diameter equal to (a) $8 \mathrm{~mm}$, (b) $10 \mathrm{~mm}$ and (c) $13 \mathrm{~mm}$. The hollow and solid symbols correspond to actual measurement using hot-film and corrected values by correcting the error of directional insensitiveness in hot-film.

There exists a saddle point along the synthetic jet centerline where the minimum velocity is zero at particular instant of time and greater than zero for rest of the time (Glezer \& Amitay, 2002). They also observed that during the suction stroke of diaphragm, reversal of velocity exists before the saddle point and the effect is maximum at orifice exit and decreases towards saddle point. Therefore, the magnitude of time-averaged velocity is minimum at the orifice exit and increases to a higher magnitude at the end of suction region (region-A in Figure 4.6 (a)). Once the vortex ring is formed and comes out from the suction region, the steadily translating vortex ring has almost constant velocity (region-B), which indicates approximately constant vorticity of vortex core with negligible loss of energy. The combined effect of growth in instability of vortex core, viscous diffusion, mixing and interaction with other vortices in the flow field leads to radial expansion of vortex core. Therefore, the velocity of the vortex ring decreases rapidly in the vortex expansion region (region-C) and finally the vortex ring breaks into a broad spectrum of small vortices that leads to plateau in velocity distribution in far field (region-D).

The time-averaged stream wise velocity component measured using hot-film along the synthetic jet centerline are shown for 8,10 and $13 \mathrm{~mm}$ orifice in Figure 4.7. The 
measured velocity is corrected for directional insensitiveness of single wire hot-film in suction region. It has been found that the effect of suction stroke is negligible after $\mathrm{X} / \mathrm{D}=$ 2 for all three orifices and at all actuation frequencies. The averaged velocity at the center of the orifice exit is greater than zero indicates that the velocity distribution at orifice exit during suction period differ from that of ejection period. In other words if $\varphi$ is phase angle of suction period for a sinusoidal velocity program then the absolute magnitude of velocity at $\varphi+\pi$ is not same at orifice exit.

The velocity profile does not show a steep rise for $8 \mathrm{~mm}$ and $10 \mathrm{~mm}$ orifice as the vortex ring remains laminar over a longer distance in downstream direction with negligible smaller scale vortices generated by trailing jet or by bursting of vortex ring (see Figure 4.3 (a) and (b)). In contrary, velocity after $\mathrm{X} / \mathrm{D}=2$ decreases immediately after attaining the maximum velocity. This is also supported by the LIF image shown in Figure 4.3 (c), where the vortex ring is distorted and partially evolve due to following suction stroke. The velocity decreases after the suction region $(\mathrm{X} / \mathrm{D}<3)$ at $2 \mathrm{~Hz}$ actuation for 8 and $10 \mathrm{~mm}$ orifice and for $13 \mathrm{~mm}$ orifice the velocity remains at higher value till $\mathrm{X} / \mathrm{D}=9$ followed by a steep decline (see Figure 4.7).

The Reynolds number increases at fixed orifice size with increase in the actuation frequency from 1 to $2 \mathrm{~Hz}$ (see Table 1). Therefore, the flow structure changes at $2 \mathrm{~Hz}$ actuation frequency in comparison to $1 \mathrm{~Hz}$ actuation frequency as shown in Figure 4.3. Synthetic jet with leading vortex ring and isolated coherent trailing jet for $8 \mathrm{~mm}$ orifice at $2 \mathrm{~Hz}$ actuation frequency (Figure 4.3 (d)) and synthetic jet with leading vortex ring but negligible trailing jet for $10 \mathrm{~mm}$ orifice at $2 \mathrm{~Hz}$ actuation frequency (Figure 4.3 (e)) propagates for a longer distance preserving their coherency. This leads to a lower rate of velocity decline after $\mathrm{X} / \mathrm{D}=3$ in Figure 4.7 (a) and (d) at $2 \mathrm{~Hz}$ actuation frequency. The 
leap forging and bursting of vortex ring is seen at $\mathrm{X} / \mathrm{D}=9$ and this is the reason for steep decline in velocity as shown in Figure 4.7 (c) at $2 \mathrm{~Hz}$ actuation frequency.

As compared to $8 \mathrm{~mm}$ orifice, the trailing jet and leading vortex rings are clearly distinguishable and coherent at 4 and $6 \mathrm{~Hz}$ actuation frequency for 10 and $13 \mathrm{~mm}$ orifice due to comparatively lower $\mathrm{L} / \mathrm{D}$ ratio (Table 4.1 ) than $8 \mathrm{~mm}$ orifice at same actuation frequency (See Figure 4.4). The deviation of vortex rings from synthetic jet centerline is more frequent after $\mathrm{X} / \mathrm{D}=4$ for $8 \mathrm{~mm}$ orifice as observed from the sequence of images. In LIF images shown in Figure 4.4 (a) and (d), a continuous interaction with the mesh of left over trailing jet for $8 \mathrm{~mm}$ orifice at 4 and $6 \mathrm{~Hz}$ actuation frequency is observed.

Both of these reasons are attributed to the peak observed near $X / D=4$ which is followed by steep decline and subsequently by a constant velocity up to $\mathrm{X} / \mathrm{D}=8$ in velocity distribution as shown in Figure 4.7 (a) for $8 \mathrm{~mm}$ orifice at 4 and $6 \mathrm{~Hz}$ actuation. In Figure 4.7 (b) for $10 \mathrm{~mm}$ orifice the peaks are followed by a clear decline of velocity (loss of coherency) at 4 and $6 \mathrm{~Hz}$ actuation frequency between $\mathrm{X} / \mathrm{D}=2$ to 4 , whereas a steady translation of vortex ring is observed by higher value of velocity up to $\mathrm{X} / \mathrm{D}=9$ to 12 at 1 and $2 \mathrm{~Hz}$ actuation frequency. For $13 \mathrm{~mm}$ orifice in Figure 4.7(c), due to coherent trailing jet and leading vortex for a longer distance in downstream direction (see Figure 4.4 (c) and (f)), the velocity distribution remains at higher state for a longer distance ranging from $\mathrm{X} / \mathrm{D}$ $=2$ to 9 for 4 and for $6 \mathrm{~Hz}$ actuation frequency respectively.

\subsubsection{Time-averaged velocity field in transverse direction}

The velocity profiles in transverse direction at different X/D locations are shown in Figure 4.8. The downstream locations are chosen such that $\mathrm{X} / \mathrm{D}=2$ corresponds to jet exit region with negligible effect of suction stroke, $X / D=5$ corresponds to the region where vortex formation is complete and have large-scale coherent structures and $\mathrm{X} / \mathrm{D}=8$ corresponds to 
the region where vortex ring is in the process of enlargement and decay. Results are shown at two additional points $\mathrm{X} / \mathrm{D}=12$ and 15 for 4 and $6 \mathrm{~Hz}$ actuation frequency. The velocity is non-dimensionalized by the maximum velocity $\left(\mathrm{U}_{\mathrm{C}}\right)$ at the respective cross-stream locations. The synthetic jet have no trailing jet or have weak trailing jet as compared to the leading vortex ring for $8 \mathrm{~mm}$ orifice actuating at 1 and $2 \mathrm{~Hz}$ actuation frequency (see Figure 4.3 (a) and (d)). The velocity distribution at 1 and $2 \mathrm{~Hz}$ actuation frequencies (see Figure 4.7 (a)), indicates that the broadening of vortex rings in downstream direction is negligible until $\mathrm{X} / \mathrm{D}=8$. This is because of self-similar nature of velocity profiles. At 4 and $6 \mathrm{~Hz}$ actuation frequency, the velocity profile is not self-similar and there is broadening of velocity profile in stream wise direction. The magnitude of peak velocity at the centerline falls rapidly (see Figure 4.7 (a) and (b)). The broadening of jet is also supported by the LIF images (see Figure 4.4).

In comparison to velocity distribution for $8 \mathrm{~mm}$ orifice at $1 \mathrm{~Hz}$ actuation frequency in Figure 4.8 (a), the spreading of jet is higher for $10 \mathrm{~mm}$ orifice in Figure 4.9 (a) as evident from flattening of velocity profile. At $2 \mathrm{~Hz}$ actuation frequency in Figure 4.9 (b), higher velocity is observed till $\mathrm{Z} / \mathrm{D}=0.6$ with more flattening of velocity as compared to $1 \mathrm{~Hz}$ actuation frequency due to increased spreading of synthetic jet as compared to that of $8 \mathrm{~mm}$ orifice. The reduced velocity and L/D ratio as compared to $8 \mathrm{~mm}$ orifice reduces the circulation strength of vortex ring.

The velocity magnitude is higher near the synthetic jet centerline and decreases in transverse direction for 8 and $10 \mathrm{~mm}$ orifice as seen in Figure 4.8 (a) and Figure 4.9 (a) at $1 \mathrm{~Hz}$ actuation frequency. However, this trend is not present in Figure 4.10 (a) at $1 \mathrm{~Hz}$ actuation frequency. This occurs due to partial suction and early breakdown of the vortex ring as visible in LIF images shown in Figure 4.3 (c). When the velocity signal is analysed for all these cases at $\mathrm{X} / \mathrm{D}=2$ in time domain the effect of suction stroke is negligible (seen 
as doubling of peaks respective to the actuation frequency which will be discussed in next section of this chapter). This value is significant only for $13 \mathrm{~mm}$ orifice with minimum at synthetic jet centerline and maximum at $\mathrm{Z} / \mathrm{D}=0.8$. The directional insensitiveness of hotfilm causes a false rise of velocity (discussed in next section of this chapter) in this region. The succeeding vortex ring which have comparatively higher speed penetrate inside the slowdown vortex ring of previous cycle and leads to the phenomena of leap forging (see Figure 4.3 (c) and (f). This can be seen as a velocity undulation or secondary peaks near $\mathrm{Z} / \mathrm{D}=1$ at $\mathrm{X} / \mathrm{D}=8$ in Figure 4.10 (a). Similar trend but with increased magnitude is also observed in Figure 4.10 (b) for $\mathrm{X} / \mathrm{D}=5$ at $\mathrm{Z} / \mathrm{D}=1.8$ and a undulation in velocity profile at $\mathrm{X} / \mathrm{D}=2$ and 8 . The LIF images and velocity profile indicate that these velocity fluctuations are due to the periodic passage of the vortex rings and not due to broad spectrum of turbulent velocity fluctuations.
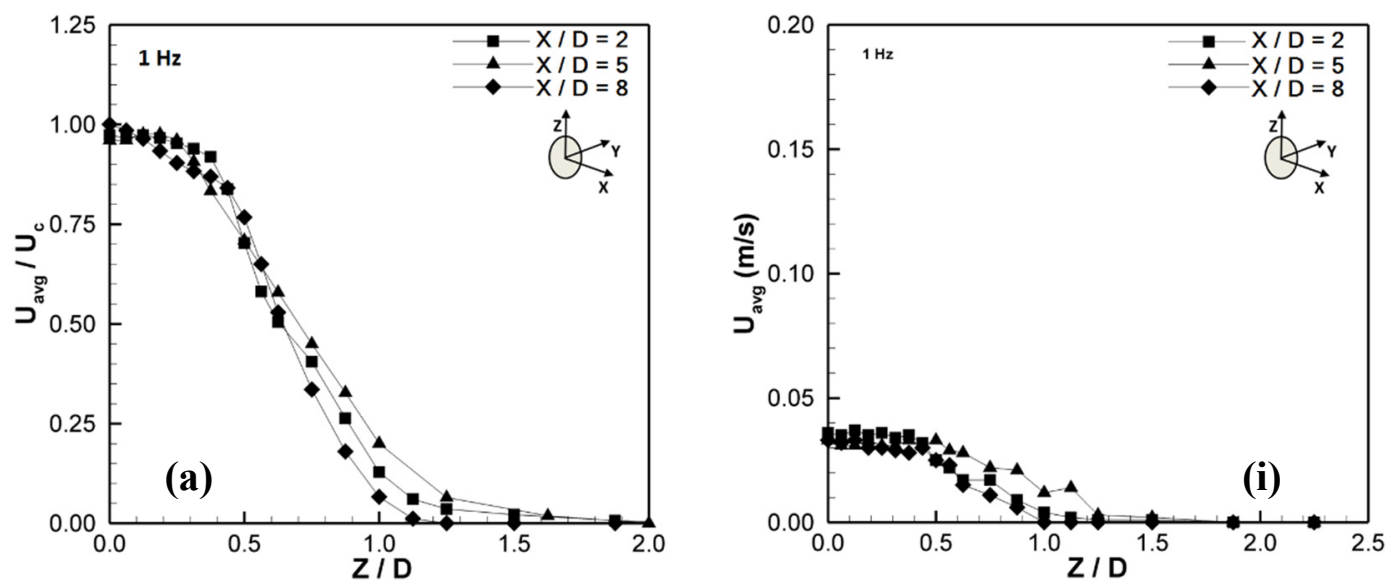

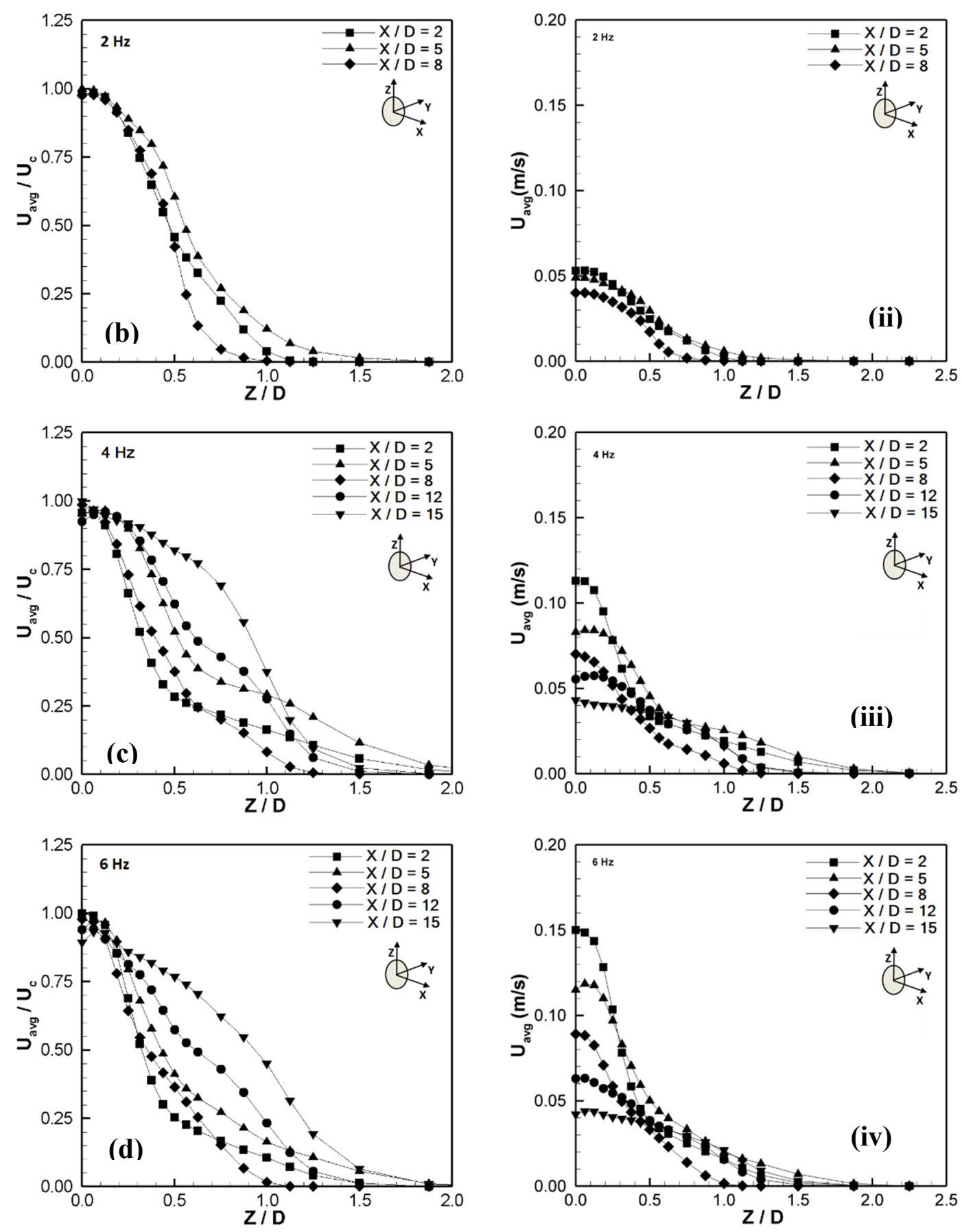

Figure 4.8: Time-averaged streamwise velocity profile on non-dimensional and actual scale for $8 \mathrm{~mm}$ orifice in transverse direction at downstream locations $X / D=2,5,8,12$ and 15 for actuation frequencies (a, i) $1 \mathrm{~Hz}$, (b, ii) $2 \mathrm{~Hz}$, (c, iii) $4 \mathrm{HZ}$ and (d, iv) $6 \mathrm{~Hz}$. 

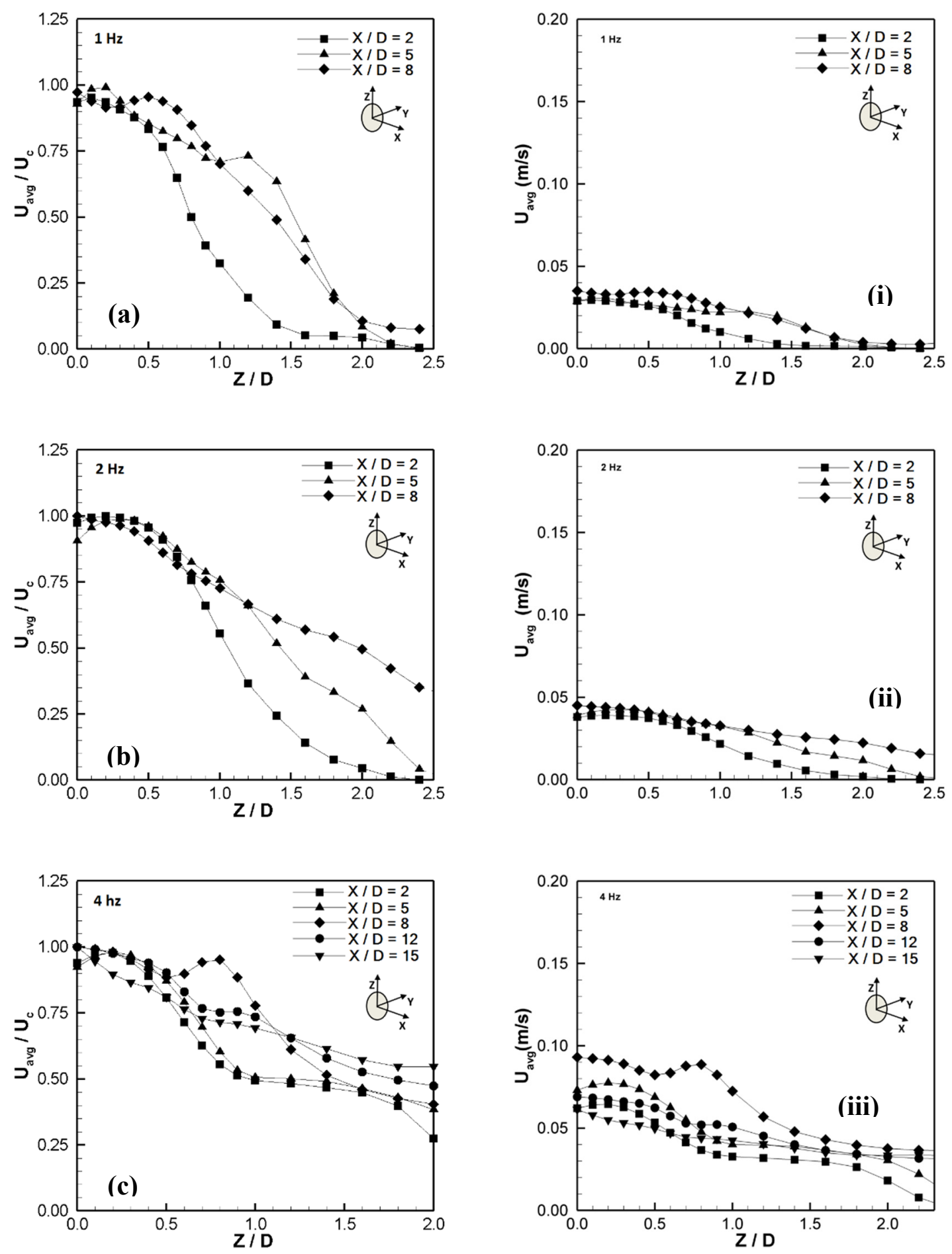

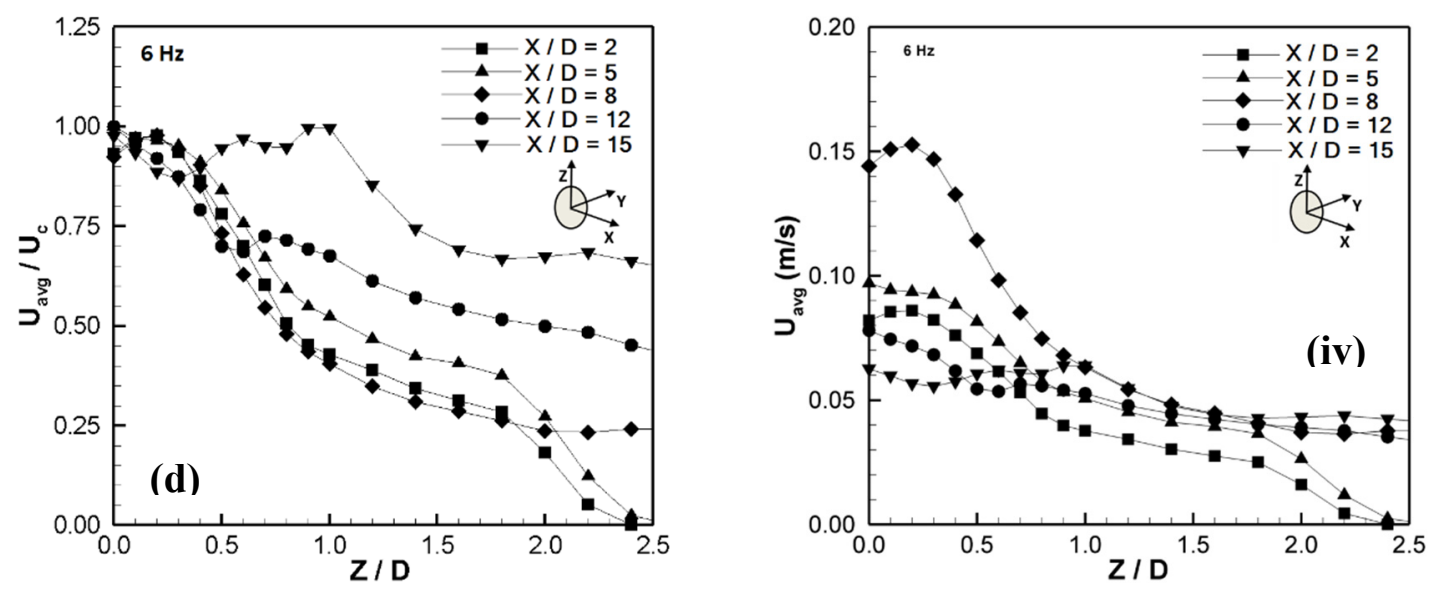

Figure 4.9: Time-averaged streamwise velocity profile on non-dimensional and actual scale for 10 $\mathrm{mm}$ orifice in transverse direction at downstream locations $X / D=2,5,8,12$ and 15 for actuation frequencies (a, i) $1 \mathrm{~Hz}$, (b, ii) $2 \mathrm{~Hz}$, (c, iii) $4 \mathrm{HZ}$ and (d, iv) $6 \mathrm{~Hz}$.


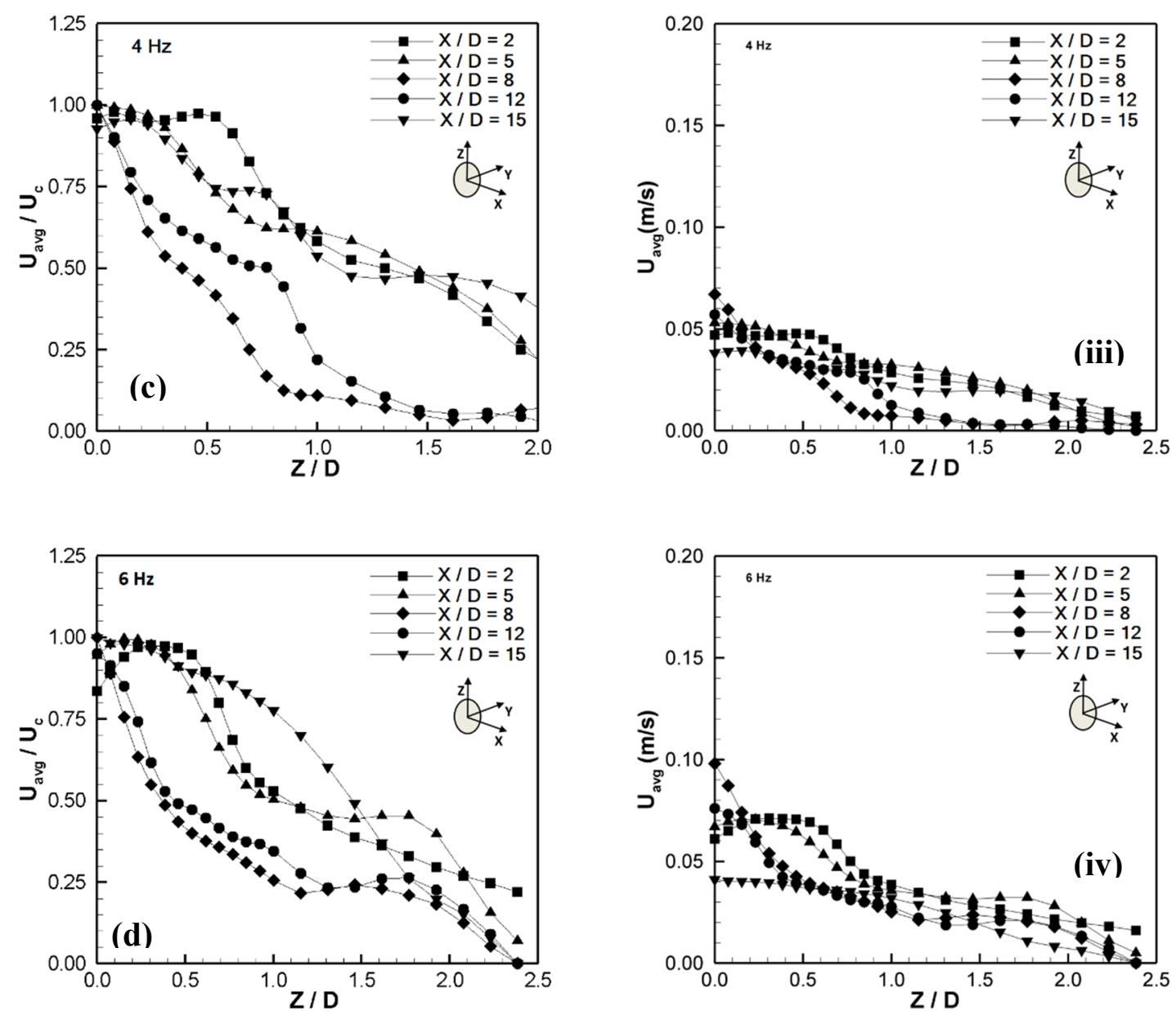

Figure 4.10: Time-averaged streamwise velocity profile on non-dimensional and actual scale for 13 $\mathrm{mm}$ orifice in transverse direction at downstream locations $X / D=2,5,8,12$ and 15 for actuation frequencies (a, i) $1 \mathrm{~Hz}$, (b, ii) $2 \mathrm{~Hz}$, (c, iii) $4 \mathrm{HZ}$ and (d, iv) $6 \mathrm{~Hz}$. 


\subsection{Synthetic jets with rectangular orifice}

The presence of corners and aspect ratio play an important role in the formation and propagation of vortex rings. Therefore, the formation number alone is not sufficient to predict the flow field of rectangular synthetic jet compared to the circular synthetic jet. Three orifices of aspect ratios equal to 1,2, and 4 are investigated at four-actuation frequencies 1, 2, 4, and $6 \mathrm{~Hz}$. This section focuses on the formation and near field behaviour of synthetic jet as a function of aspect ratio and actuation frequency. Actuation frequency and diaphragm displacement are same as specified for circular orifices. The hydraulic diameter $\left(\mathrm{D}_{\mathrm{h}}\right)$ is kept constant for all three non-circular orifices and is equal to $12 \mathrm{~mm}$. For all three aspect ratios of 1, 2 and 4, the formation number $\left(L / D_{h}\right)$ based on slug length $(L)$ varies between the range 1.1 to 4.5 .

\subsubsection{Flow visualization}

The LIF flow visualization images have been used to study the structure of the vortex ring generated by the synthetic jet. Two orthogonal planes (XZ \& XY) have been chosen to for the visualization. Due to symmetricity of AR1 orifice, the LIF image is captured at single plane (XZ-plane) and shown in Figure 4.11. For AR2 and AR4 orifices, the LIF images have been captured in both XZ and XY-planes and are shown in Figure 4.12 and Figure

\subsection{3 respectively.}

In Figure 4.11(a) for AR1 orifice at $1 \mathrm{~Hz}$ actuation frequency, the leading vortex ring sheds a very weak trailing jet which is consumed by the following leading vortex ring. The vortex rings are well separated and coherent in nature. At $2 \mathrm{~Hz}$ actuation frequency in Figure 4.11(b), the synthetic jet initiates considerable amount of shedding behind the leading vortex ring and the region between the consecutive leading vortex rings is filled by the sparse distribution of trailing jet. The trailing jet are incoherent in nature and interacts 
with the incoming vortex rings. At 4 and $6 \mathrm{~Hz}$ actuation frequencies, the vortex rings are more diffused and show stronger trailing jet.
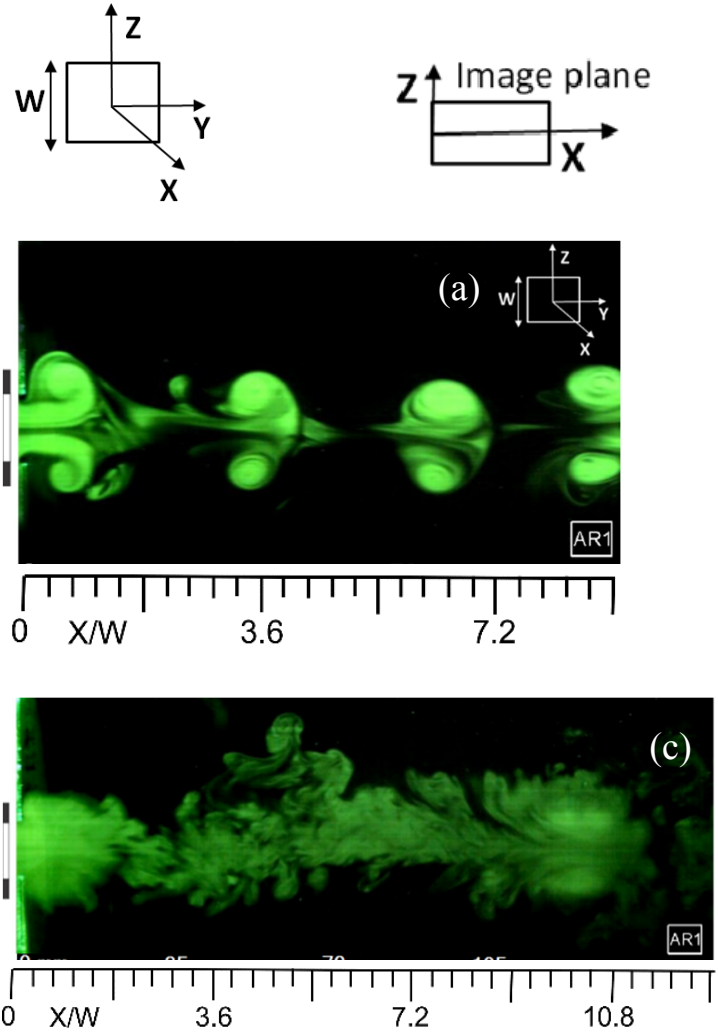


Figure 4.11: Flow visualization images of synthetic jet in XZ-plane (side view) for square orifice (AR1) at (a) $\mathrm{f}=1 \mathrm{~Hz}, \mathrm{~L} / \mathrm{D}_{\mathrm{h}}=1.6, \mathrm{Re}=\mathbf{2 4 2}$, (b) $\mathrm{f}=2 \mathrm{~Hz}, \mathrm{~L} / \mathrm{D}_{\mathrm{h}}=\mathbf{2 . 3}, \mathrm{Re}=553$, (c) $\mathrm{f}=4 \mathrm{~Hz}, \mathrm{~L} / \mathrm{D}_{\mathrm{h}}=$ 3.3, $R e=1742$, and (d) $f=6 \mathrm{~Hz}, \mathrm{~L} / \mathrm{D}_{\mathrm{h}}=4.5, \mathrm{Re}=3846$.
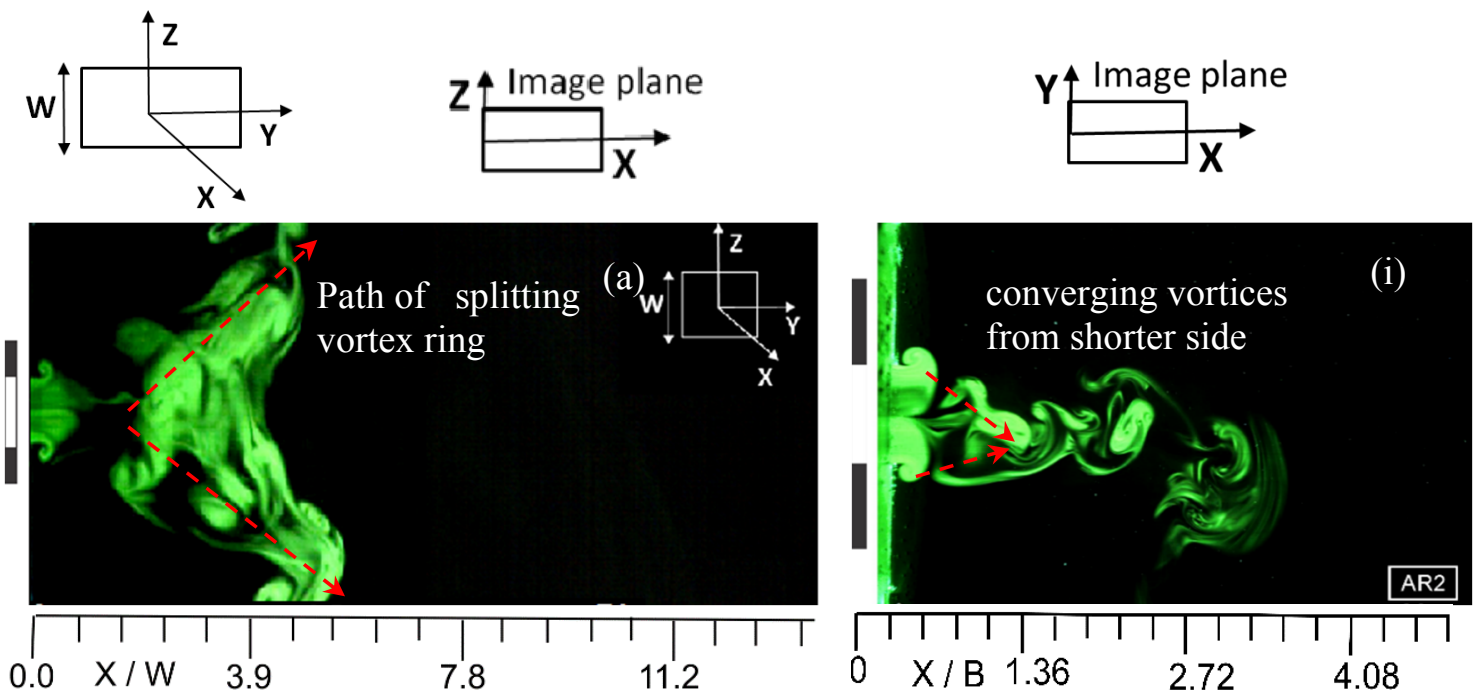

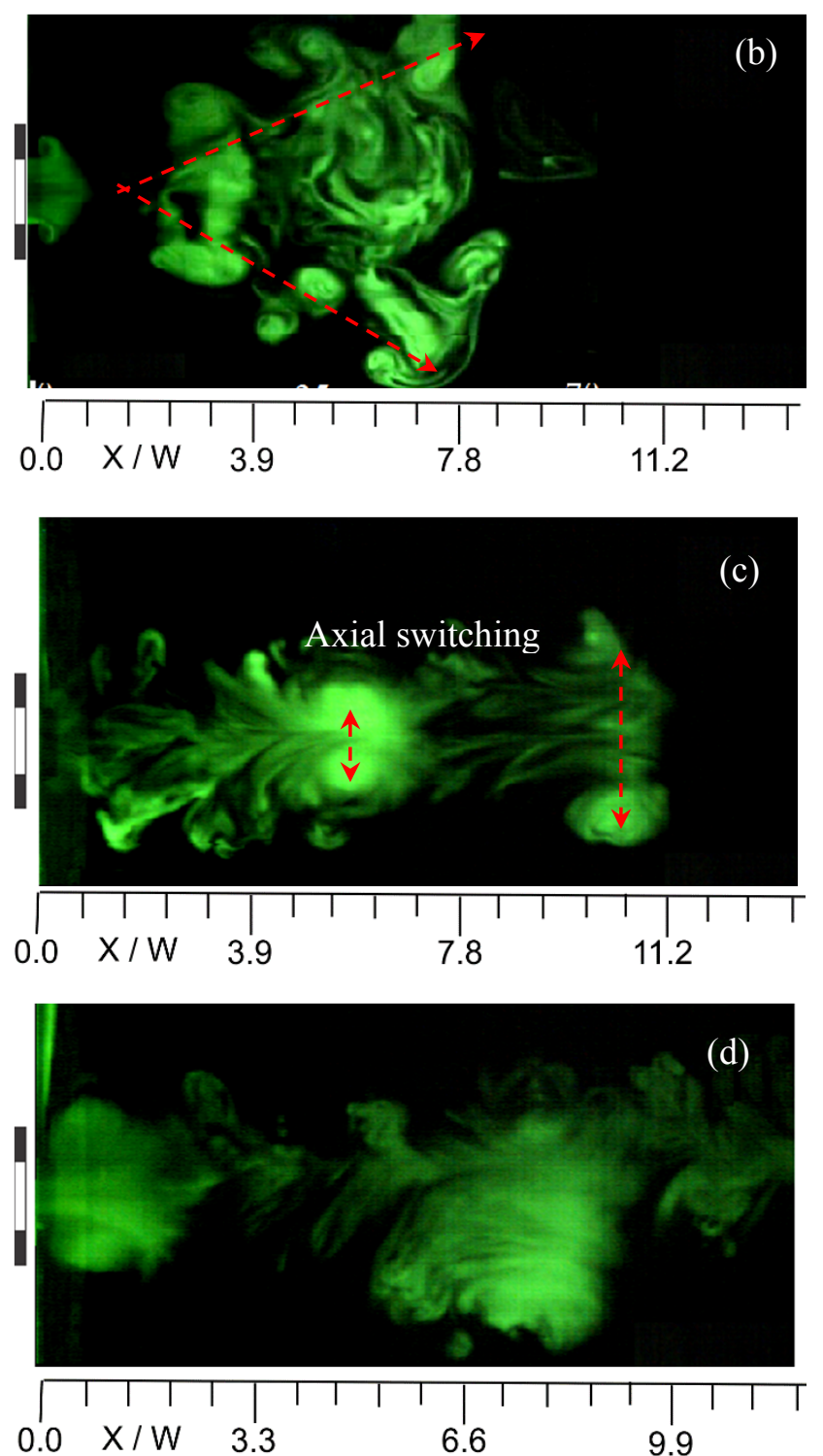
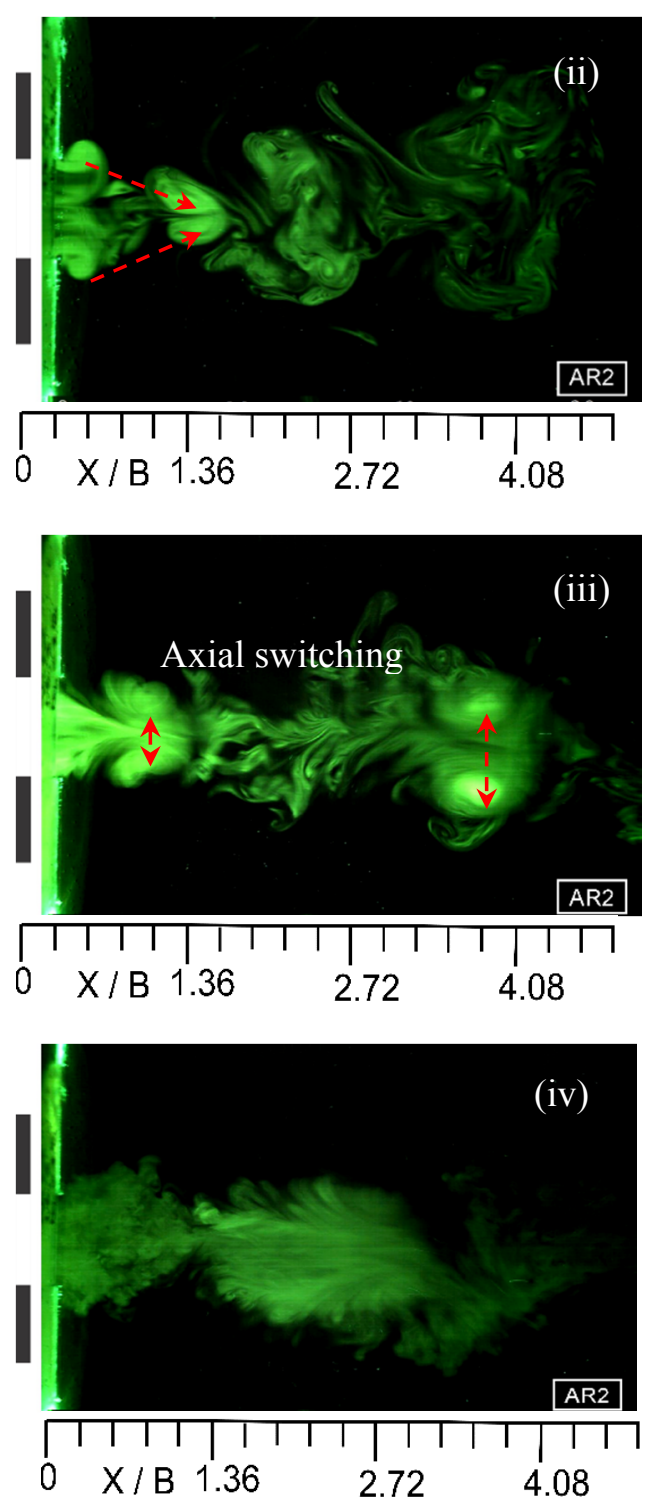

Figure 4.12: Flow visualization images of synthetic jet in XZ-plane (side view) and in XY-plane (top view) for rectangular shaped orifice (AR2) at ((a) \&(i)) $f=1 \mathrm{~Hz}, \mathrm{~L} / \mathrm{D}_{\mathrm{h}}=1.5, \mathrm{Re}=215$, ((b) \& (ii)) at $f=2 \mathrm{~Hz}, \mathrm{~L} / \mathrm{D}_{\mathrm{h}}=\mathbf{2 . 1}, \mathrm{Re}=491,\left((\mathrm{c}) \&\right.$ (iii)) $\mathrm{f}=4 \mathrm{~Hz}, \mathrm{~L} / \mathrm{D}_{\mathrm{h}}=2.9, \operatorname{Re}=1549$ and ((d) \& (iv)) $\mathrm{f}=6$ $\mathrm{Hz}, \mathrm{L} / \mathrm{D}_{\mathrm{h}}=4, \mathrm{Re}=3435$. (* The images in $\mathrm{XY}$ and $\mathrm{XZ}$-planes are not at same time instant). 

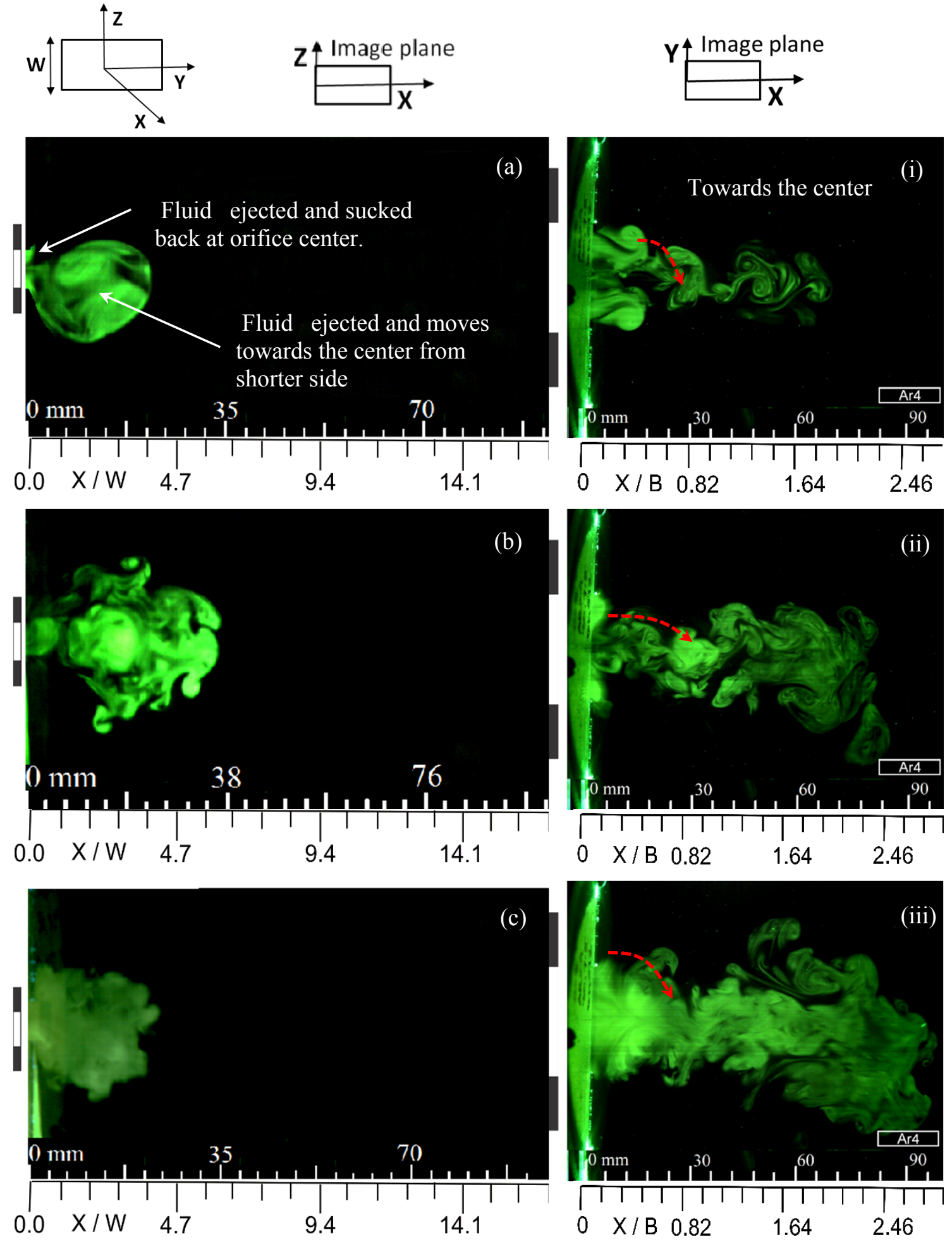


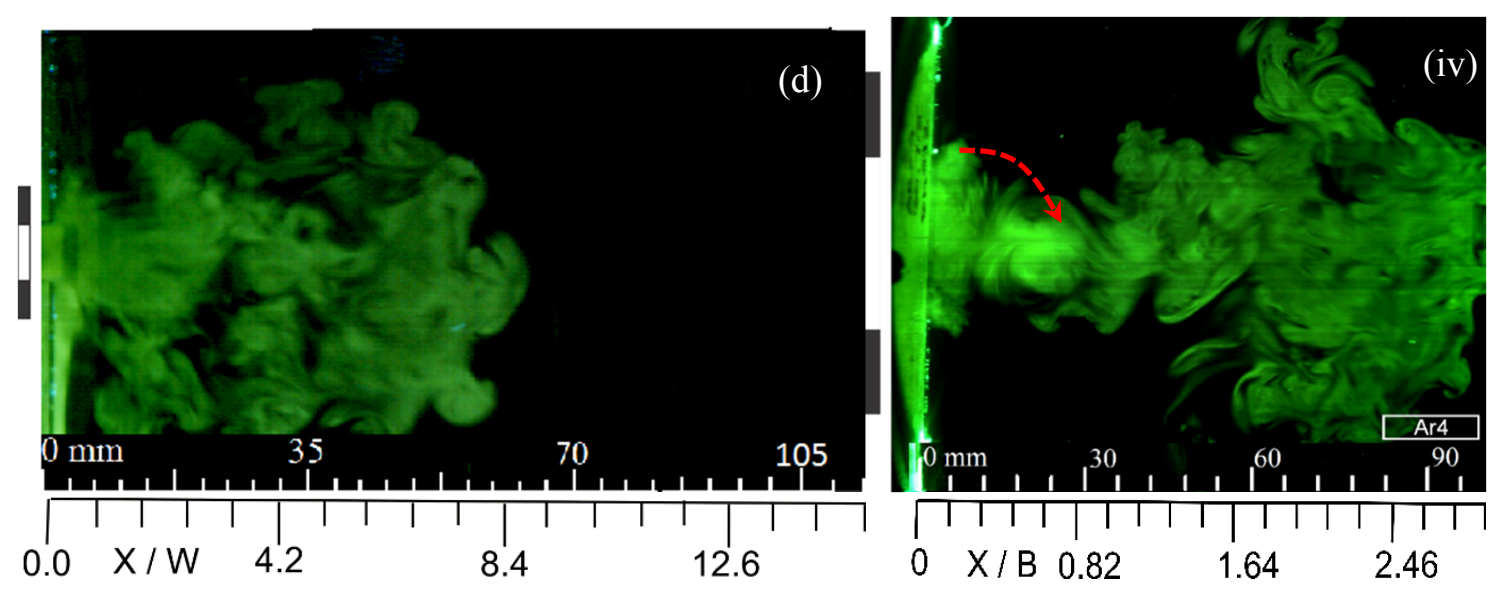

Figure 4.13: Flow visualization images of synthetic jet in XZ-plane (side view) and in XY-plane (top view) for rectangular shape orifice (AR4) at ((a) \& i)) $f=1 \mathrm{~Hz}, \mathrm{~L} / \mathrm{D}_{\mathrm{h}}=1.1, \mathrm{Re}=155$, ((b) \& (ii)) at $\mathrm{f}=2 \mathrm{~Hz}, \mathrm{~L} / \mathrm{D}_{\mathrm{h}}=1.5, \operatorname{Re}=354$ ((c) \& (iii)) $\mathrm{f}=4 \mathrm{~Hz}, \mathrm{~L} / \mathrm{D}_{\mathrm{h}}=2.1, \operatorname{Re}=1115$ and ((d) \& (iv)) f=6 Hz, $\mathrm{L} / \mathrm{D}_{\mathrm{h}}=2.9, \mathrm{Re}=2473$. ( $* *$ The images in $\mathrm{XY}$ and $\mathrm{XZ}$-planes are not at same time instant).

In Figure 4.12 (a) and (b) for an aspect ratio of two, the single vortex ring splits itself into two isolated vortex rings and convects at an angle of 72 degree to each other. The splitting of vortex ring is observed in a plane perpendicular to the longer side (in XZ-plane) near $\mathrm{X} / \mathrm{W}=1.5$ from the orifice exit. Approximately, at the same downstream distance the merging of counter rotating vortices are seen in Figure 4.12 (i). Similar trend of vortex splitting and merging of counter rotating vortices are also observed at $2 \mathrm{~Hz}$ actuation frequency in Figure 4.12 (b) and (ii). The difference between the two cases results in the reduction of splitting angle from $72^{0}$ to $52^{0}$ for $2 \mathrm{~Hz}$ actuation frequency. Another difference is the portion of vortex ring left between the splited vortex rings. At 4 and $6 \mathrm{~Hz}$ actuation frequency, the axial switching is indicated from the variation of separation distance between counter rotating vortices in two orthogonal planes as discussed in subsequent sections. For AR4 orifice at $1 \mathrm{~Hz}$ actuation frequency, the portion of ejected vorticity is sucked back towards the cavity as shown in Figure 4.13 (a) and (i). The segment of vortex ring from shorter side has comparatively higher induced velocity due to increased curvature and thus the segment of vortex ring on shorter side is able to move downstream and not sucked back into the cavity. The ejected fluid near the center of longer side of 
orifice is sucked back inside the orifice cavity (see Figure 4.13 (a), (i)). Similar trend of flow structure is also observed at $2 \mathrm{~Hz}$ actuation frequency. However, due to increased velocity and frequency, the chunk of vorticity moving towards the center from shorter side gets brusted and this can be seen as smaller segments of vortices in Figure 4.13 (b) and (ii). The synthetic jet for AR4 orifice at 4 and $6 \mathrm{~Hz}$ actuation frequency as shown in Figure 4.13 (c, d, iii and iv) breaks into smaller pieces in their early stage of the vortex formation and thus no recognizable vortex ring is observed in the LIF images. The spreading and mixing of synthetic jet is quite high for AR4 orifice as compared to AR1 and AR2 orifices in near field region. The uneven influence of suction stroke on forming vortex ring leads to early breakdown of vortex ring into small vortices. The other possible factor is the large difference in the self-induced velocity of the vortex segment ejecting near the shorter and longer side of orifice. The flow patterns for this aspect ratio orifice are more chaotic leading to higher spreading and mixing with the surroundings.

(a)
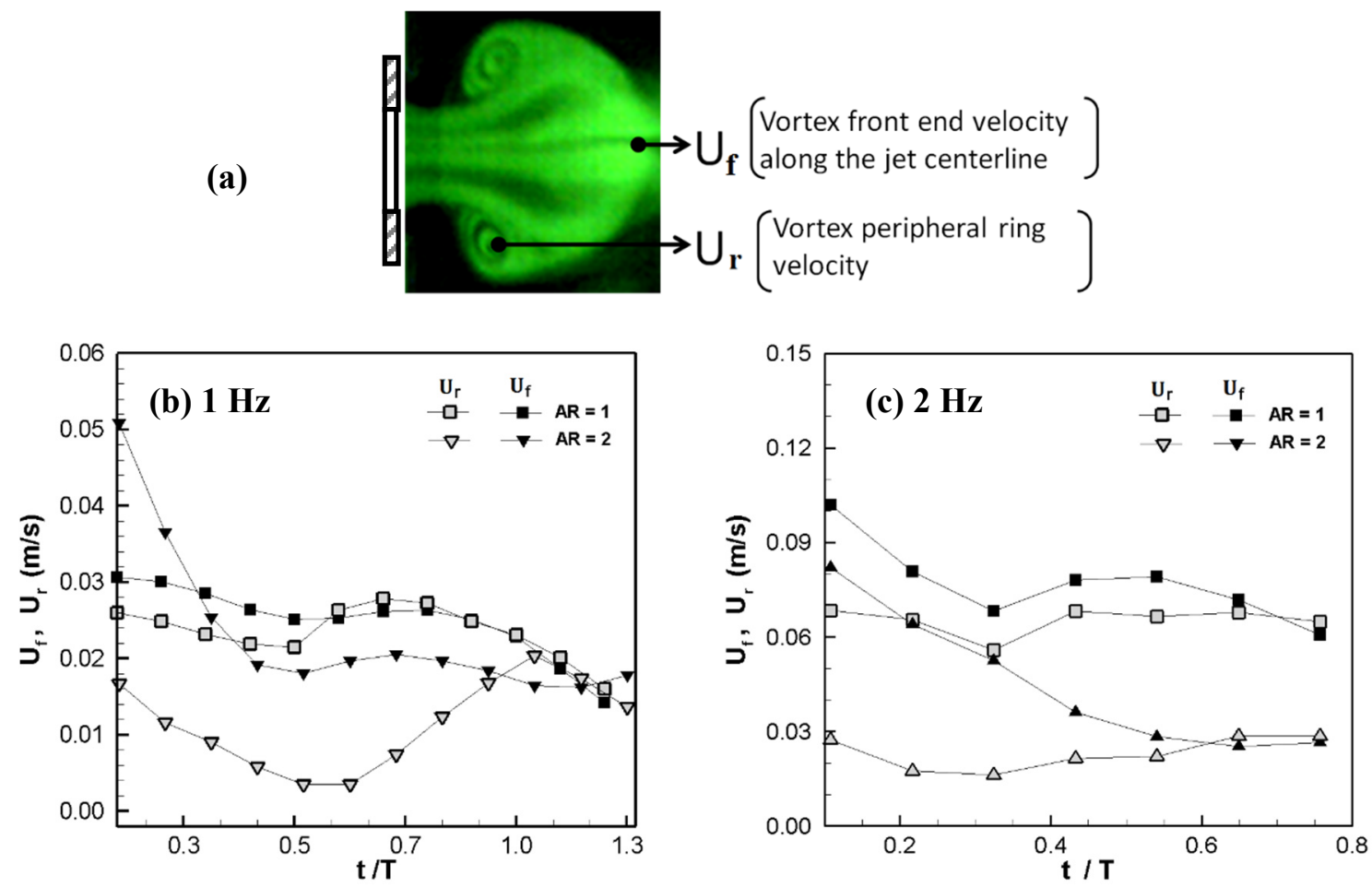

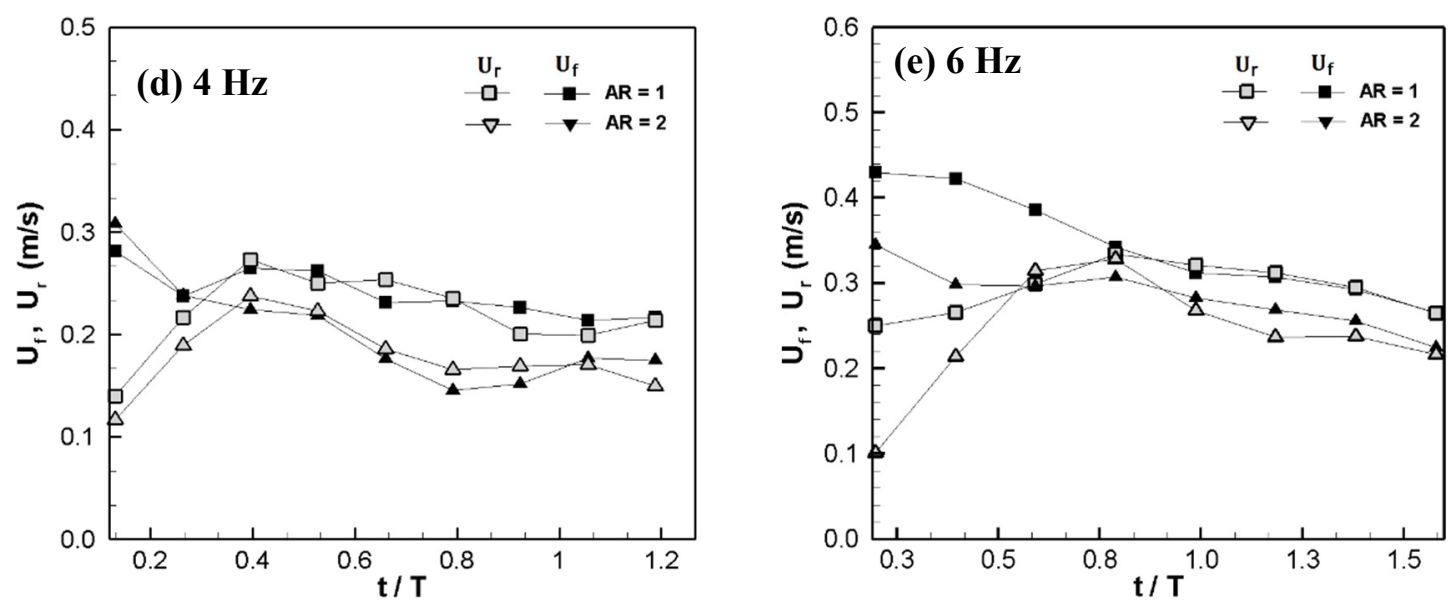

Figure 4.14: (a) Stream wise velocity of front end of leading vortex ring $\left(U_{f}\right)$ and counter rotating vortices of leading vortex ring $\left(U_{r}\right)$ calculated from the sequence of LIF images for AR1 and AR2 orifices in XZ-plane for (b) $1 \mathrm{~Hz}$, (c) $2 \mathrm{~Hz}$, (d) $4 \mathrm{~Hz}$ and (e) $6 \mathrm{~Hz}$ actuation frequency.

For obtaining more insight into the axial switching and bifurcation process, the relationship between the velocity of vortex front-end and vortex-core (peripheral ring or tube of vortex ring) as shown in Figure 4.14 (a) has been explored. The velocity difference between vortex front-end $\left(\mathrm{U}_{\mathrm{f}}\right)$ and vortex-core $\left(\mathrm{U}_{\mathrm{r}}\right)$ has been calculated using the sequence of LIF images and shown in Figure 4.14. The approximate time duration between each frame is obtained using frame grabbing rate and the corresponding velocity is calculated from travelled distance extracted from the LIF images (for detail see appendix B). The variation in $U_{f}$ and $U_{r}$ for AR1 and AR2 orifice at four frequencies 1, 2, 4 and $6 \mathrm{~Hz}$ are shown in Figure 4.14 (b, c, d, and e). Since, there is early breakdown of vortex ring and mixing in AR4 orifice as shown in Figure 4.13, it is not possible to distinguish the front end and peripheral ring velocity and hence not shown.

The sequence of LIF images reveals that the difference between $U_{f}$ and $U_{r}$ play an important role in the bifurcation of vortex ring as a large difference in velocity leads to larger stretching of vortex rings. In Figure 4.14(b) for AR1 orifice at $1 \mathrm{~Hz}$ excitation frequency, initially the magnitude of $U_{f}$ is larger than $U_{r}$ between $t / T=0.1$ to 0.5 and the two values are close to each other at $t / T=0.5$. At latter time i.e. at $t / T=0.6$, the two values 
differs with opposite trend and then converge at $t / T=0.9$. After $t / T=0.9$, both $U_{f}$ and $U_{r}$ differ with very low margin. The alternate sign of variation in the magnitude of $U_{f}$ and $U_{r}$ corresponds to increase and decrease in the velocity of vortex ring due to axial switching. The AR2 orifice at $1 \mathrm{~Hz}$ actuation frequency shows a larger difference between the magnitude of $U_{f}$ and $U_{r}$ between $t / T=0.1$ to 0.9 . They cross each other after $t / T=0.9$ and proceeds with reduced difference between the two. Here the vortex ring ejected from rectangular shaped orifice is not able to switch its axis until a longer duration and downstream distance. This stretches the vortex ring outwards in XZ-plane and contracts in XY-plane and finally the vortex rings bifurcates into two rings. These bifurcated vortex rings moves further at an angle to each other (see Figure 4.12 (a) and (b)). At $2 \mathrm{~Hz}$ actuation frequency in Figure 4.14 (c) for AR1 orifice, $U_{f}$ remains higher for a longer duration till $\mathrm{t} / \mathrm{T}=0.7$, but the bifurcation is not observed. Typically, a square vortex ring experiences $45^{\circ}$ of axial switching due to symmetricity of corners (Grinstein et al., 1995 and Gohil et al., 2010). The AR2 orifice case at $2 \mathrm{~Hz}$ actuation follow the same trend of $1 \mathrm{~Hz}$ actuation, but the convergence period falls between $\mathrm{t} / \mathrm{T}=0.55$ to 0.65 . The differences in magnitude of $\mathrm{U}_{\mathrm{f}}$ and $\mathrm{U}_{\mathrm{r}}$ in Figure 4.14 (d) and (e) for AR1 and AR2 are more significant before $\mathrm{t} / \mathrm{T}=$ 0.4 for actuation frequency $4 \mathrm{~Hz}$ and before $\mathrm{t} / \mathrm{T}=0.8$ for $6 \mathrm{~Hz}$ actuation frequency, thereafter the difference is either negative or negligible.

The axial switching for square orifice is not significant in comparison to higher aspect ratio orifices, because for AR1 orifice all the corners are at equal distance from the orifice center. Depending on the ratio of major to minor axis, the vortex ring undergoes many changes in its pattern prior to axial switching. The portions of the vortex ring with higher curvature convect ahead of the rest due to higher local self-induced velocity. The portion of vortex ring, which lags behind, stretches the vortex ring and drags the fast moving corner portions towards the center. The fast moving segments of vortex ring 
towards the centre pushes the slow moving portion (middle portion) of vortex ring outward, which increases its curvature by reducing its length. The reduced length of longer side of vortex ring is added to shorter side, which increases the length of shorter side. Consequently, the major axis becomes minor and minor axis becomes major. This switching is repeated as the vortex ring convects downstream until its breakdown, well known as axial switching of vortex ring. Hussain et al. (1989) explained axial switching for excited and non-excited elliptical plain jet based on difference in velocities of different segments of vortex ring. The non-circular vortex ring are curvature dependent and the selfadvection velocity of the vortical structures produces more three dimensional deformation as compared to the circular normal vortex ring. The vortex splitting which is also known as bifurcation was reported by Auerbach and Grimm (1994) while experimenting on rectangular shaped vortex rings ejected from the nozzle of aspect ratio in the range of 2 to 20. They reported the bifurcation for a rectangular shaped vortex ring having aspect ratio three. These results are concentrated on the formation of single vortex ring without backward stroke. In the second part, the study is again repeated for a single forward stroke followed by a backward stroke. For different piston velocity profile and stroke length, the non-splitting motion of primary vortex ring is reported for first case and splitting of primary vortex ring is reported for second case. It was concluded that it is the backward stroke, which renders a significant influence on the splitting of vortex ring. Synthetic jet is also a cyclic repetition of forward and backward stroke of movable boundary, where the backward stroke influences the vortex formation and its stability in downstream direction. 


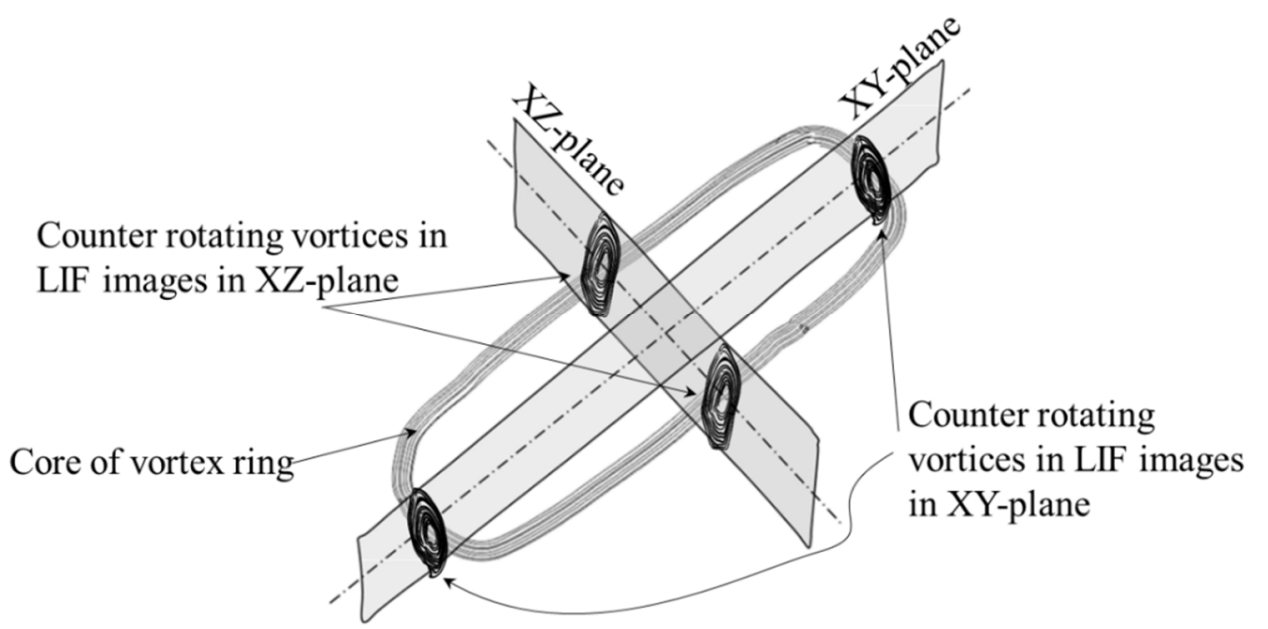

Figure 4.15: Schematic representation of the counter rotating vortices in major (XY) and minor (XZ) axis planes for non-circular vortex ring.

As the axial switching of vortex ring has substantial impact on fluid entrainment and flow structure, the number of its occurrence before the breakdown of vortex ring have great importance. The separation distance between the counter rotating vortices increases and decreases with time. The contraction of vortex ring in XY-plane towards the synthetic jet centerline decreases the separation distance between the counter rotating vortices in XYplane. The movement of vortex ring towards the center in XY-plane pushes the vortex tube outwards in Z-direction and thus increase in separation distance between the counter rotating vortices in the XZ-plane is observed. Figure 4.16 shows minor variation in the separation distance of counter rotating vortices in downstream direction in $\mathrm{XY}$ and $\mathrm{XZ}$ planes, which indicates that either the axial switching is absent or the orthogonal planes are not sufficient to capture the event. Because, Gohil et al. (2010) reported a 45 degree of axial switching for square shaped vortex ring whereas Grinstein et al. (1995) reported that if the ratio of circular-equivalent diameter to momentum thickness at the side of the orifice is small, the continuous square jet is not expected to have any axial switching. 

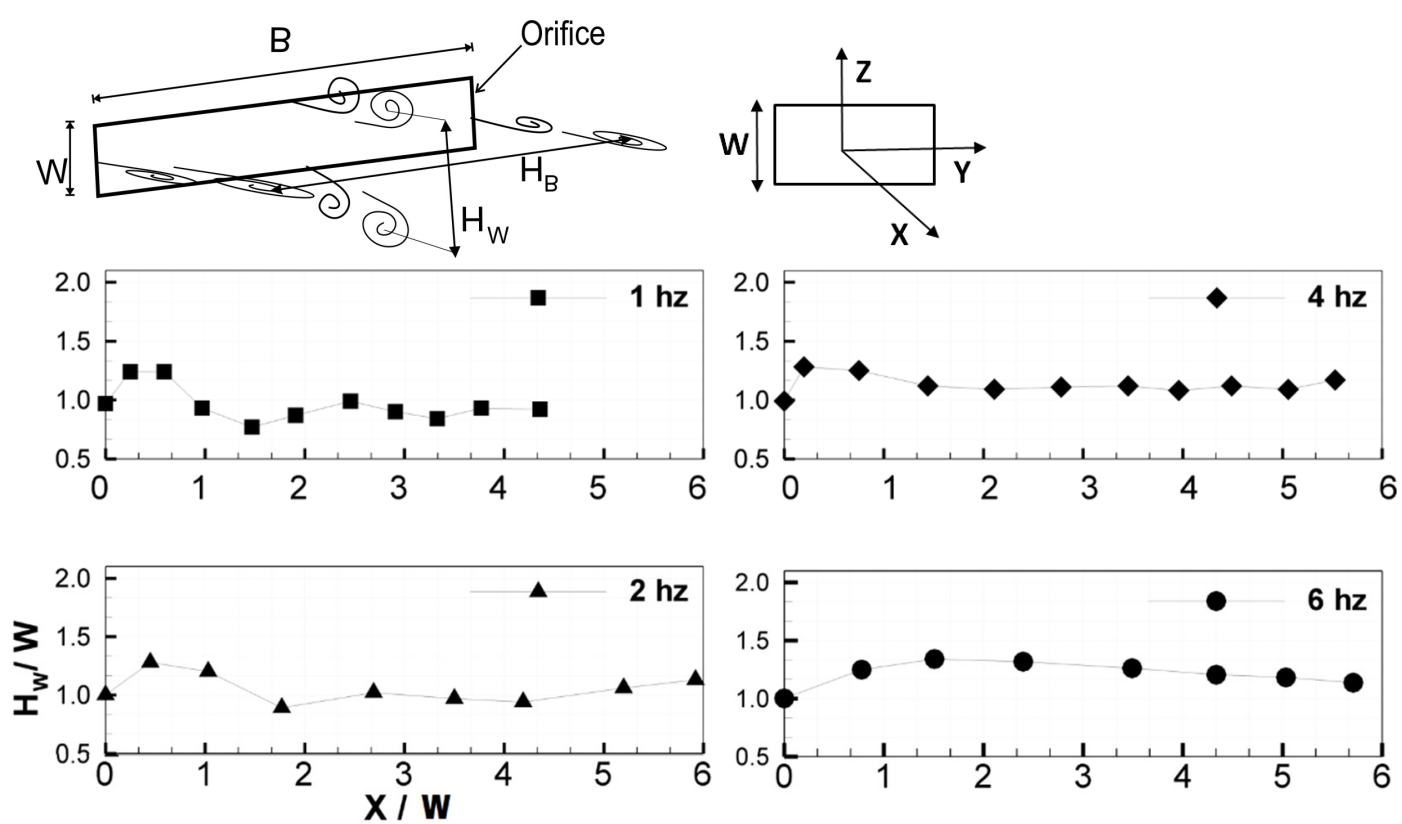

(a) AR1


(b) AR2
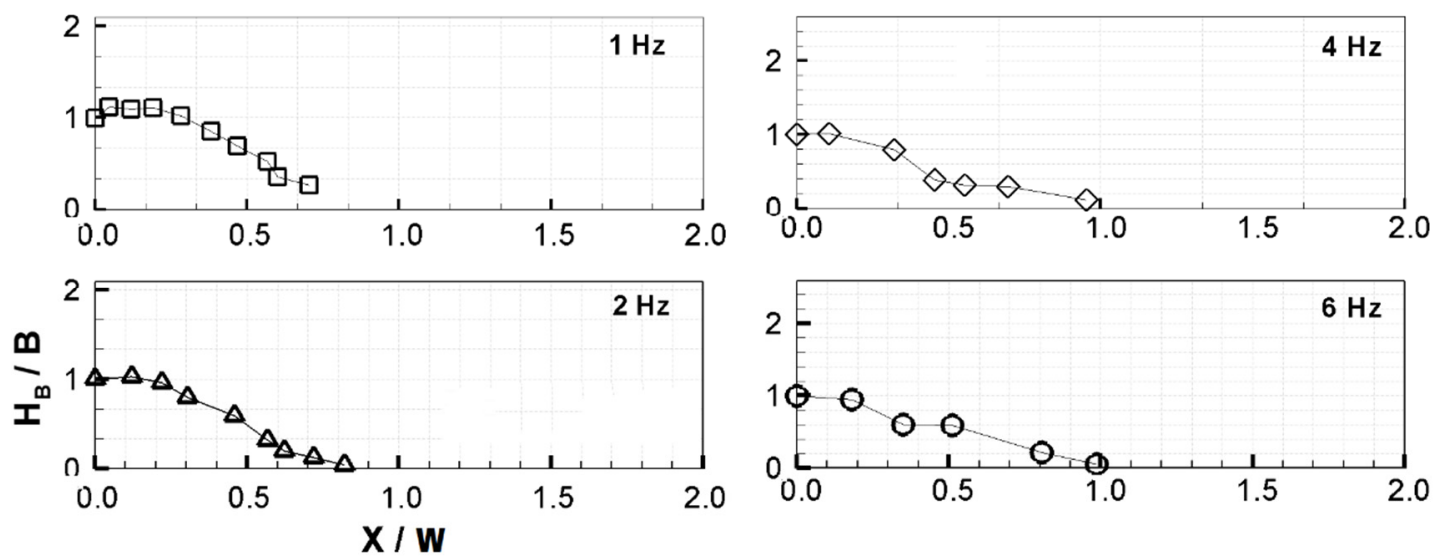

(c) AR4

Figure 4.16: Separation distance between the counter rotating vortices in LIF images captured in XY and XZ-plane at actuation frequency 1, 2, 4 and $6 \mathrm{~Hz}$ for (a) AR1 (b) AR2 and (c) AR4 orifice cases. 
The separation distance between the counter rotating vortices for AR2 orifice is shown in Figure 4.16 (b). The variation in the size of vortex ring follows entirely different trend for axial switching and bifurcation process. The $\mathrm{H}_{\mathrm{w}}$ and $\mathrm{H}_{\mathrm{B}}$ values crosses each other at $\mathrm{X} / \mathrm{W}=0.6$ and $\mathrm{X} / \mathrm{W}=0.8$ for 1 and $2 \mathrm{~Hz}$ actuation frequency respectively. After the vortex overshoot $(\mathrm{X} / \mathrm{W}>0.3)$, the value of $\mathrm{HW}$ remains constant in between $\mathrm{X} / \mathrm{W}=0.3$ to 1.5 at 1 and $2 \mathrm{~Hz}$ actuation frequency. However, the $\mathrm{H}_{\mathrm{B}}$ decreases rapidly within this region and finally disappears as the counter rotating vortices merge together. As soon as the counter rotating vortices merge in XY-plane and vortex ring splits in XZ-plane (see Figure 4.12 (i) \& (ii)), the magnitude of $\mathrm{H}_{w}$ increases rapidly (after vortex splitting $\mathrm{H}_{\mathrm{w}}$ is measured between the top and bottom vortices). At 4 and $6 \mathrm{~Hz}$ actuation frequency, $\mathrm{H}_{\mathrm{B}}$ and $\mathrm{Hw}_{\mathrm{w}}$ flip their values twice and in half way of third attempt the vortex rings breaks. The flipping of $\mathrm{H}_{\mathrm{W}}$ and $\mathrm{H}_{\mathrm{B}}$ represents the completion of axial switching of vortex ring. The first axial switching is completed near $\mathrm{X} / \mathrm{W}=2$ and the second axial switching between near $\mathrm{X} / \mathrm{W}=$ 4.4 at $4 \mathrm{~Hz}$ actuation frequency. The attempt of third axial switching is also observed between $\mathrm{X} / \mathrm{W}=4.4$ to 7 before vortex breakup. At $6 \mathrm{~Hz}$, the limited frame grabbing rate restricts to identify the clear locations of axial switching but the approximate location is somewhere between $\mathrm{X} / \mathrm{W}=1.8$ to 2.2 for first axial switching and between $\mathrm{X} / \mathrm{W}=3.5$ to 4 for second axial switching.

The separation distance between the counter rotating vortices are difficult to measure from LIF images in XZ-plane (see Figure 4.3 and Figure 4.4) for AR4 orifice due early breakdown, mixing and ambiguity in identifying the counter rotating vortices. Therefore, the separation distance $\left(\mathrm{H}_{\mathrm{B}}\right)$ is measured only in XY-plane are shown in Figure 4.16 (c). At 1 and $2 \mathrm{~Hz}$ actuation frequency, the magnitude of $\mathrm{H}_{\mathrm{B}}$ approaches to zero near $\mathrm{X} / \mathrm{W}=0.65$ and 0.85 respectively, whereas at 4 and $6 \mathrm{~Hz}$ actuation frequency $\mathrm{HB}$ approached to zero near $\mathrm{X} / \mathrm{W}=1$. The trend of variation for $\mathrm{HB}$ at 4 and $6 \mathrm{~Hz}$ actuation 
frequency is similar to the observation obtained for the bifurcation cases at 1 and $2 \mathrm{~Hz}$ actuation frequency for AR2 orifice. Instead of splitting, the forming vortex ring blooms very close to the orifice and spread very quickly in XZ-plane in the form of tiny vortices. The increased spreading in $\mathrm{XY}$-plane is observed after $\mathrm{H}_{\mathrm{B}}$ is almost zero or negligible.

\subsubsection{Time-averaged flow field}
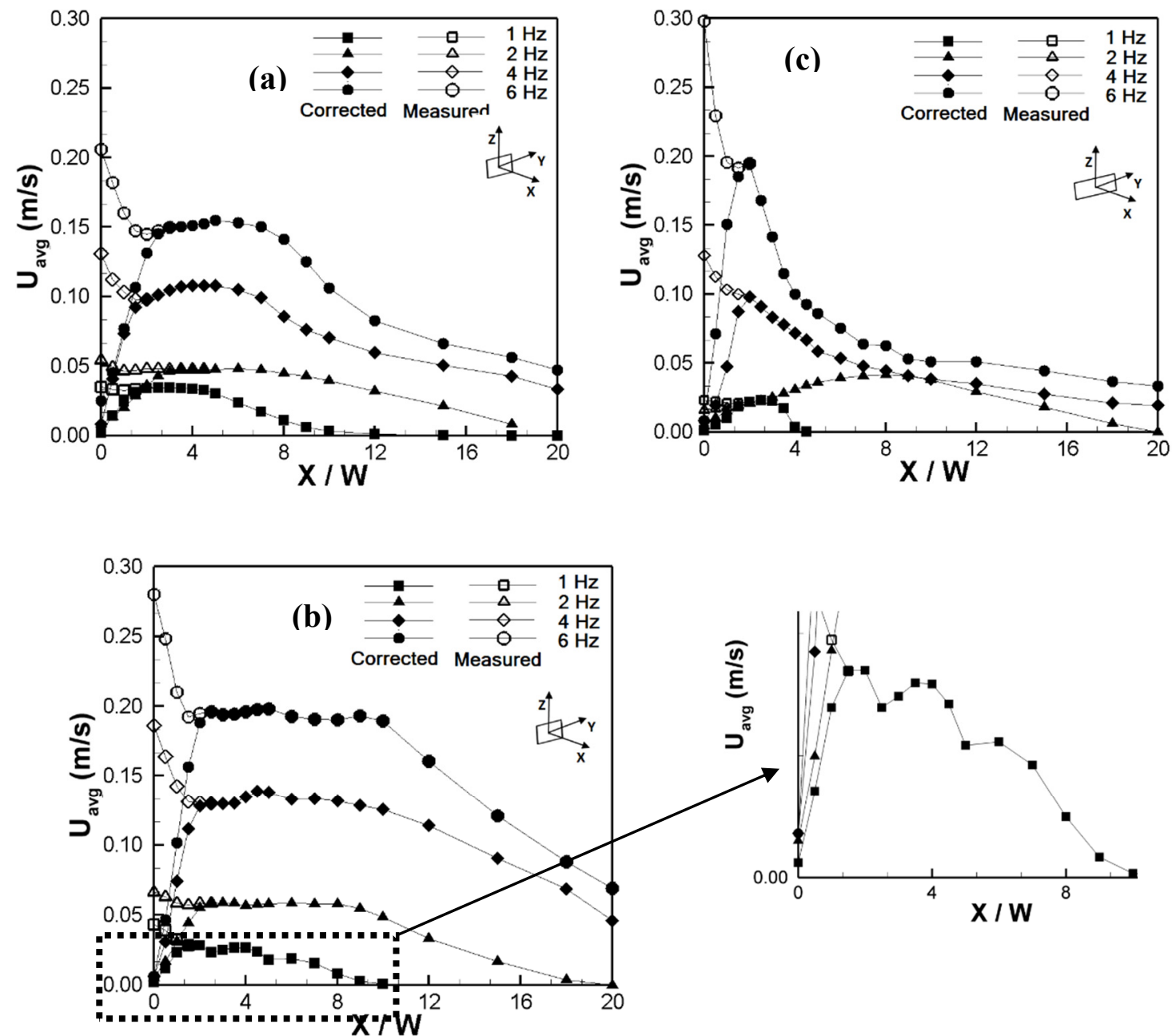

Figure 4.17: Time-averaged streamwise velocity measured along the synthetic jet centerline at different downstream locations actuating at 1, 2, 4 and $6 \mathrm{~Hz}$ actuation frequency for rectangular shaped orifices: (a) AR1, (b) AR2 and (c) AR4. (*The hollow and solid symbols correspond to actual measurement using hot film and corrected values by correcting the error of directional insensitiveness in hot film).

Figure 4.17 show the time-averaged streamwise velocity distribution in downstream direction along the synthetic jet centerline for rectangular orifices of aspect ratio AR1, AR2 
and AR4. The measured error due to directional insensitiveness of hot-film has been corrected and shown. The corrected average velocity is calculated by subtracting the suction region from the exit velocity region for a minimum of 30 actuation cycles. Both the corrected and measured velocity distributions are presented as solid and hollow symbols respectively. Assuming the symmetry of orifice in both Y and Z-direction, the velocity profile is plotted only in Y-direction for AR1 orifice. By close examination of the velocity signal at various actuation frequency and axial locations, it has been observed that suction velocity becomes negligible beyond $\mathrm{X} / \mathrm{W}=3$ for all cases studied in the present investigation.

The velocity increases rapidly from the orifice exit to saddle point (discussed in 4.3.3) and during this period the shear layer ejecting out from the orifice exit rolled up and forms the vortex ring. In Figure 4.17 (a) for AR1 orifice at $1 \mathrm{~Hz}$ excitation frequency, the average velocity increases in the streamwise direction till $\mathrm{X} / \mathrm{W}=2$ and remains constant till $\mathrm{X} / \mathrm{W}=4.5$ followed by a slow reduction till $\mathrm{X} / \mathrm{W}=7$ and finally after some distance the vortex rings disappears. At $2 \mathrm{~Hz}$ actuation frequency the velocity increases to a higher value and remains constants till $\mathrm{X} / \mathrm{W}=8$ and then decreases slowly in downstream direction. The slow decay rate of velocity after attaining higher state of velocity at both the frequencies 1 and $2 \mathrm{~Hz}$ is an indication of slow mixing of vortex ring with the surroundings. The average velocity of AR1 at $4 \mathrm{~Hz}$ actuation frequency increases in the streamwise direction until $\mathrm{X} / \mathrm{W}=3$ (suction zone), remains at higher value up to $\mathrm{X} / \mathrm{W}=5$ indicating a steadily translating vortex ring which is followed by a rapid reduction of velocity. Because of vortex growth, the velocity falls rapidly between $\mathrm{X} / \mathrm{W}=5$ to $\mathrm{X} / \mathrm{W}=10$, and after breakdown finally decreases slowly due to viscous dissipation. The velocity distribution at $6 \mathrm{~Hz}$ actuation follow the same trend of $4 \mathrm{~Hz}$ actuation with increased vortex ring translation at higher state. 
The enlarged view of centerline velocity profile for AR2 orifice at $1 \mathrm{~Hz}$ excitation frequency in Figure 4.17 (b) shows an undulation of velocity profile in between $\mathrm{X} / \mathrm{W}=0$ to 6. A sudden fall in velocity magnitude occurs after $\mathrm{X} / \mathrm{W}=1.5$ due to the vortex ring growth (see Figure 4.12 (a)). The formation of secondary peaks is observed at $\mathrm{X} / \mathrm{W}=4$ near the vortex splitting region. After $\mathrm{X} / \mathrm{W}=6$, the bifurcated vortex ring moves away from the synthetic jet centerline as seen in Figure 4.12 (b) and a very steep negative slope is observed in Figure 4.16. The cross-sectional area of AR2 $\left(162 \mathrm{~mm}^{2}\right)$ is larger than that of AR1 $\left(144 \mathrm{~mm}^{2}\right)$, but the magnitude of maximum velocity is larger for AR2 orifice as compared to the AR1 orifice 4 and $6 \mathrm{~Hz}$ actuation frequency (compare Figure 4.17 (a) and (b)). The possible reason is that a change in orifice aspect ratio subsequently changes the velocity distribution at orifice exit and further in downstream. As discussed earlier, the vortices ejecting at shorter side of orifice moves faster than the central region of vortex ring due to higher curvature. This favours an increase in suction of ambient fluid near the shorter side of orifice as compared to the central region of orifice where the vortex ring is in the formation stage. It also reduces the effect of suction stroke at the core or central region of forming vortex ring (see the vortex formation at orifice exit in Figure 4.12 (a) \& (i), where the central region is ahead or back w.r.t. to orifice sides). Another possible reason could be the length scale of axial switching, which controls the amplitude and distribution of velocity fluctuation in the downstream. An increase in actuation frequency changes the spacing between the consecutive vortex rings. The formation of trailing jet or closely spaced vortex rings may also increase or decrease the time mean velocity. As discussed earlier, since the drifting of vortex ring from the synthetic jet centerline is a function of various factors which differs from case to case also affects the time-averaged value of velocity along the synthetic jet centerline. 
In Figure 4.17 (c) for AR4 at $1 \mathrm{~Hz}$ excitation frequency, a short duration of higher velocity indicates that the mid portion of vortex ring is not able to roll up completely and moves away from the orifice before the suction strokes begins. The strong effect of suction stroke ceases the formation of vortex ring and its detachment from the orifice. The portion of vortex ring from shorter side moves toward the center and accumulate near the orifice exit. Due to increased accumulation of fluid near the orifice, the velocity increases till X/W $=2$ and then follow a steep decline in velocity. At other actuating frequencies of 2, 4 and $6 \mathrm{~Hz}$, instead of forming a single vortex ring like AR1 and AR2 orifice case, a cluster of tiny vortex rings are formed in the near field of synthetic jet ( see Figure 4.13) for AR4 orifice case. There is a gradual increase in velocity at $2 \mathrm{~Hz}$ actuation frequency till $\mathrm{X} / \mathrm{W}=$ 8 , due to the momentum gain by the ejecting fluid during ejection stroke and not due to the presence of vortex rings. At 4 and $6 \mathrm{~Hz}$ actuation frequency for AR4 orifice, the velocity increases rapidly till $\mathrm{X} / \mathrm{W}=1.2$ with subsequent reduction due to early breakup of vortex rings near the jet exit. After $\mathrm{X} / \mathrm{W}=5$, a sharp decrease in velocity is observed indicating large mixing and energy dissipation.

The streamwise velocity profile at different span wise locations at actuation frequencies 1, 2, 4, and $6 \mathrm{~Hz}$ have been presented in Figure 4.18, Figure 4.19 and Figure 4.20 for aspect ratio AR1, AR2 and AR4 respectively. The streamwise velocity is normalized by maximum streamwise velocity $\left(\mathrm{U}_{\mathrm{C}}\right)$ at the respective downstream location. The velocity of AR1 orifice is shown in a XY-plane and the velocity distribution for aspect ratio AR2 and AR4 are shown in both XY and XZ-plane. 
Table 4.4: Values of maximum streamwise velocity $\left(U_{c}\right)$ in $\mathrm{m} / \mathrm{s}$ at different cross stream locations and frequencies for AR1 orifice.

\begin{tabular}{|c|c|c|c|c|}
\hline \multirow{2}{*}{$\mathbf{X} / \mathbf{W}$} & \multicolumn{4}{|c|}{ XZ-plane } \\
\cline { 2 - 5 } & $\mathbf{1 ~ H z}$ & $\mathbf{2 ~ H z}$ & $\mathbf{4 ~ H z}$ & $\mathbf{6 ~ H z}$ \\
\hline 1 & 0.024 & 0.020 & 0.072 & 0.078 \\
\hline 3 & 0.035 & 0.046 & 0.105 & 0.149 \\
\hline 5 & 0.030 & 0.048 & 0.108 & 0.155 \\
\hline 10 & - & - & 0.070 & 0.107 \\
\hline
\end{tabular}
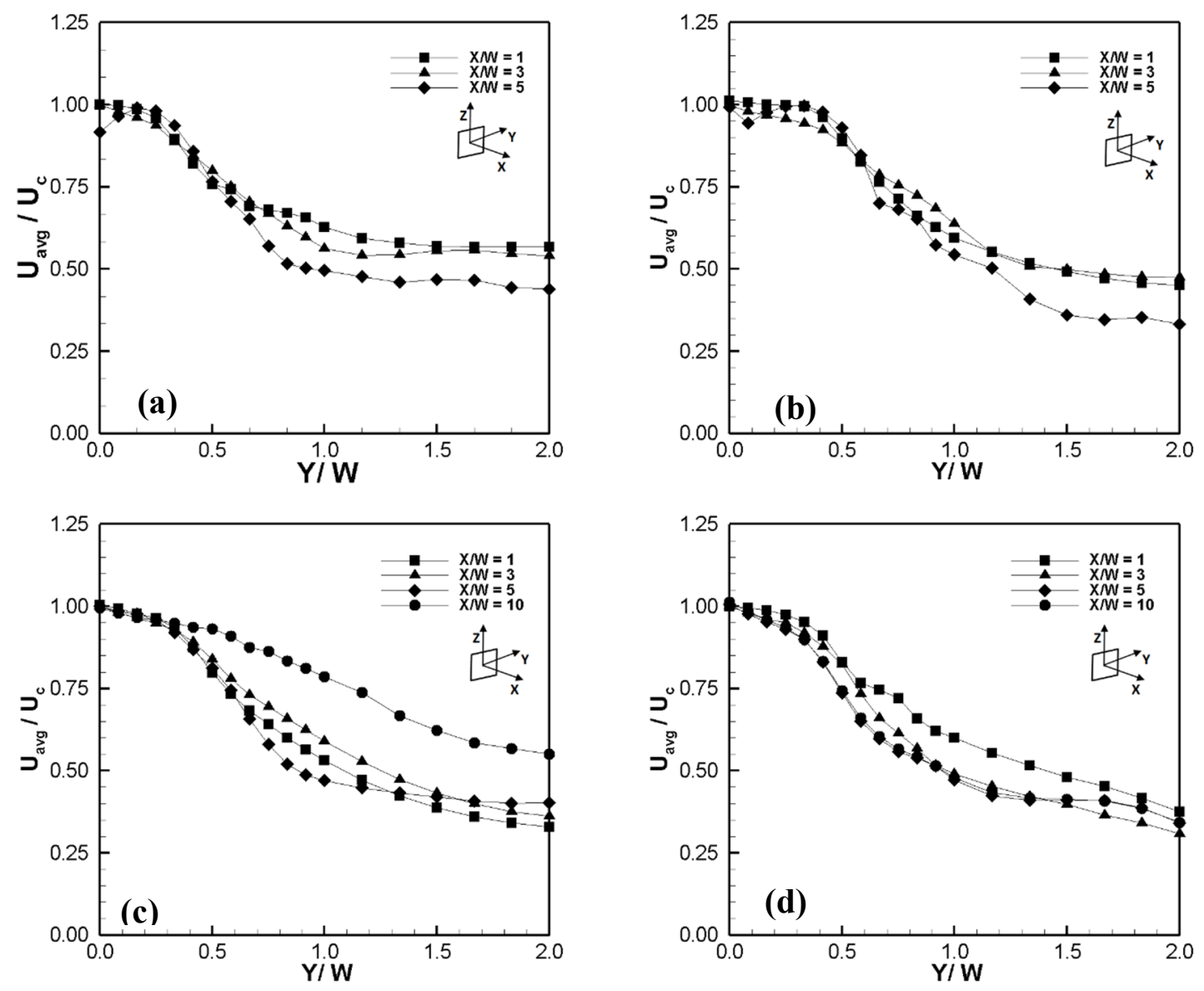

Figure 4.18: Time-averaged streamwise velocity profile in transverse direction (Y-axis) at different downstream locations $\mathrm{X} / \mathrm{W}=1,3,5$ and 10 at (a) $1 \mathrm{~Hz}$, (b) $2 \mathrm{~Hz}$, (c) $4 \mathrm{~Hz}$ and (d) $6 \mathrm{~Hz}$ actuation frequency for square orifice (AR1).

The velocity distribution for AR1 orifice shown in Figure 4.18 at different span wise locations indicates the self-similarity of velocity profile at all four frequencies. The higher velocity magnitude exist in traverse direction up to $\mathrm{Y} / \mathrm{W}=0.5$ which is followed by steeper downward slope between $\mathrm{Y} / \mathrm{W}=0.5$ to 1 for AR1 orifice at all locations due to 
increased interaction of synthetic jet outer periphery with surroundings. After $\mathrm{Y} / \mathrm{W}=1$, the monotonic decrease in velocity profile indicates a higher dissipation of energy. The overall pattern of velocity profile at all frequencies indicates the propagation of vortex ring to a longer distance with minimum entrainment of surroundings, which resembles with the LIF images shown in Figure 4.3.

In Figure 4.19, the variation in velocity profile at different downstream locations indicates that the velocity is not self-similar for AR2 orifice. The velocity distribution in XZ-plane at $1 \mathrm{~Hz}$ actuation frequency in Figure 4.19 (a) correlate with the bifurcation image of synthetic jet for AR2 orifice in Figure 4.12 (a). The location of peak shifts from Z/W = 0.4 at $\mathrm{X} / \mathrm{W}=1$ location to $\mathrm{Z} / \mathrm{W}=0.5$ at $\mathrm{X} / \mathrm{W}=3$ and at $\mathrm{Z} / \mathrm{W}=1.6$ at location $\mathrm{X} / \mathrm{W}=5$ in XZ-plane as shown in Figure 4.19 (a). In Y-direction, rapid fall in the magnitude of velocity is seen near the synthetic jet centerline at all downstream locations due to bifurcation of synthetic jet in Z-direction (see Figure 4.12(b)) which is similar to the observation in Figure 4.12 (a) at $1 \mathrm{~Hz}$ actuation frequency. At $2 \mathrm{~Hz}$ actuation frequency in Figure 4.19 (b), the velocity profile in $\mathrm{Y}$-direction at $\mathrm{X} / \mathrm{W}=1$ and 3 locations decreases more than the velocity profile at $\mathrm{X} / \mathrm{W}=5$. A higher value of velocity until $\mathrm{Y} / \mathrm{B}=0.5$ confirms the increased spreading of synthetic jet after $\mathrm{X} / \mathrm{W}=3$ in $\mathrm{Y}$-direction. In Z-direction the higher value of velocity is observed until $\mathrm{Z} / \mathrm{W}=1.0$ at $\mathrm{X} / \mathrm{W}=1$ location whereas an increased decay is observed at $\mathrm{X} / \mathrm{W}=3$ location. Therefore, it supports the bifurcation of vortex ring (see Figure 4.12(b)). At $4 \mathrm{~Hz}$ actuation frequency in Figure 4.19 (c), the velocity is minimum at $\mathrm{Y} / \mathrm{B}=0$ and increases up to $\mathrm{Y} / \mathrm{B}=0.4$. This is attributed to higher induce velocity near the shorter side of orifice that increases the time average velocity. In Z-direction, there is a gradual decrease in the velocity profile at all locations. Similarly, at $6 \mathrm{~Hz}$ actuation frequency in $\mathrm{Y}$ and Z-direction, the velocity profile has a gradual decrease. The reason is that at both 4 and $6 \mathrm{~Hz}$ actuation frequency, trailing jet is present behind the leading vortex 
ring and the flow structure is coherent (see Figure 4.12). A chunk of small-scale vortices is also present near the orifice, which interacts with the forming vortex ring.

The lower value of formation number $\left(\mathrm{L} / \mathrm{D}_{\mathrm{h}}=1.16\right)$ and aspect ratio limits the circulation of vortex ring at the longer side and the suction of ejected fluid occurs at the center of orifice for AR4 orifice at $1 \mathrm{~Hz}$ actuation frequency as shown in Figure 4.13 (i)). The suction effect at center of orifice and higher induced velocity at shorter side of orifice replicate themselves as low velocity near the synthetic jet centerline and maximum value at $\mathrm{Y} / \mathrm{B}=0.5$ for $\mathrm{X} / \mathrm{W}=1$ location (Figure 4.20 (a)). At same location $\mathrm{X} / \mathrm{W}=1$, the velocity profile decreases slowly in Z-direction. The axial switching is indicated by higher velocity at location $\mathrm{X} / \mathrm{W}=3$ in Z-direction coupled with decreasing slope in Y-direction (see Figure 4.20). Instead of completing axial switching the vortex ring breaks and spread more in XZplane as compared to XY plane (see Figure 4.13 (a) \& (i)). In Figure 4.20 (b) at location $\mathrm{X} / \mathrm{W}=3$ the velocity magnitude is high till $\mathrm{Z} / \mathrm{W}=1.2$ and shifts at $\mathrm{Z} / \mathrm{W}=1.5$ at $\mathrm{X} / \mathrm{W}=5$ location. In Y-direction, the velocity increases only at $\mathrm{Y} / \mathrm{B}=0.5$ i.e. near the orifice whereas at locations $\mathrm{X} / \mathrm{W}=3$ and 5 it decreases. This indicates that more spreading of synthetic jet in XZ-plane, which is also seen in the flow field of AR4 orifice in Figure 4.13 (b) \& (ii). At $4 \mathrm{~Hz}$ actuation frequency, the velocity distribution increases from centerline to $\mathrm{Y} / \mathrm{B}=0.5$ at location $\mathrm{X} / \mathrm{W}=1$ followed by a sudden drop (see Figure 4.20 (c)). Because the portion of vortex ring emerging from the shorter side of orifice dragged towards the vortex center due to higher induced velocity and vortex stretching. In Z-direction, the decay of velocity is observed at location $\mathrm{X} / \mathrm{W}=1$. However, due to vortex bursting and spreading (see Figure 4.13 (c) \& (iii)) the velocity remains higher for a longer distance at other locations. AT $6 \mathrm{~Hz}$ actuation frequency in Figure 4.20 (d), the trend of variation is similar to that of $4 \mathrm{~Hz}$ actuation frequency. At 2, 4 and $6 \mathrm{~Hz}$ actuation frequency, a uniform 
distribution of smaller scale vortices after vortex breakup gives rise to a straight velocity profile except very near to the orifice at $\mathrm{X} / \mathrm{W}=1$.

Table 4.5: Values of maximum streamwise velocity component at cross stream location $\left(U_{c}\right)$ in $\mathrm{m} / \mathrm{s}$ for AR2 orifice

\begin{tabular}{|c|c|c|c|c|c|c|c|c|c|}
\hline \multirow{3}{*}{$\mathrm{X} / \mathrm{W}$} & \multicolumn{4}{|c|}{ XZ-plane } & & \multicolumn{4}{|c|}{ XY-plane } \\
\hline & $1 \mathrm{~Hz}$ & $2 \mathrm{~Hz}$ & $4 \mathrm{~Hz}$ & $6 \mathrm{~Hz}$ & & $1 \mathrm{~Hz}$ & $2 \mathrm{~Hz}$ & $4 \mathrm{~Hz}$ & $6 \mathrm{~Hz}$ \\
\hline & & & & & & & & & \\
\hline 1 & 0.024 & 0.031 & 0.0746 & 0.102 & 1 & 0.023 & 0.031 & 0.089 & 0.102 \\
\hline 3 & 0.027 & 0.059 & 0.130 & 0.194 & 3 & 0.024 & 0.059 & 0.130 & 0.194 \\
\hline 5 & 0.020 & 0.058 & 0.137 & 0.197 & 5 & 0.023 & 0.058 & 0.137 & 0.197 \\
\hline 10 & - & - & 0.125 & 0.188 & 10 & - & - & 0.125 & 0.188 \\
\hline
\end{tabular}

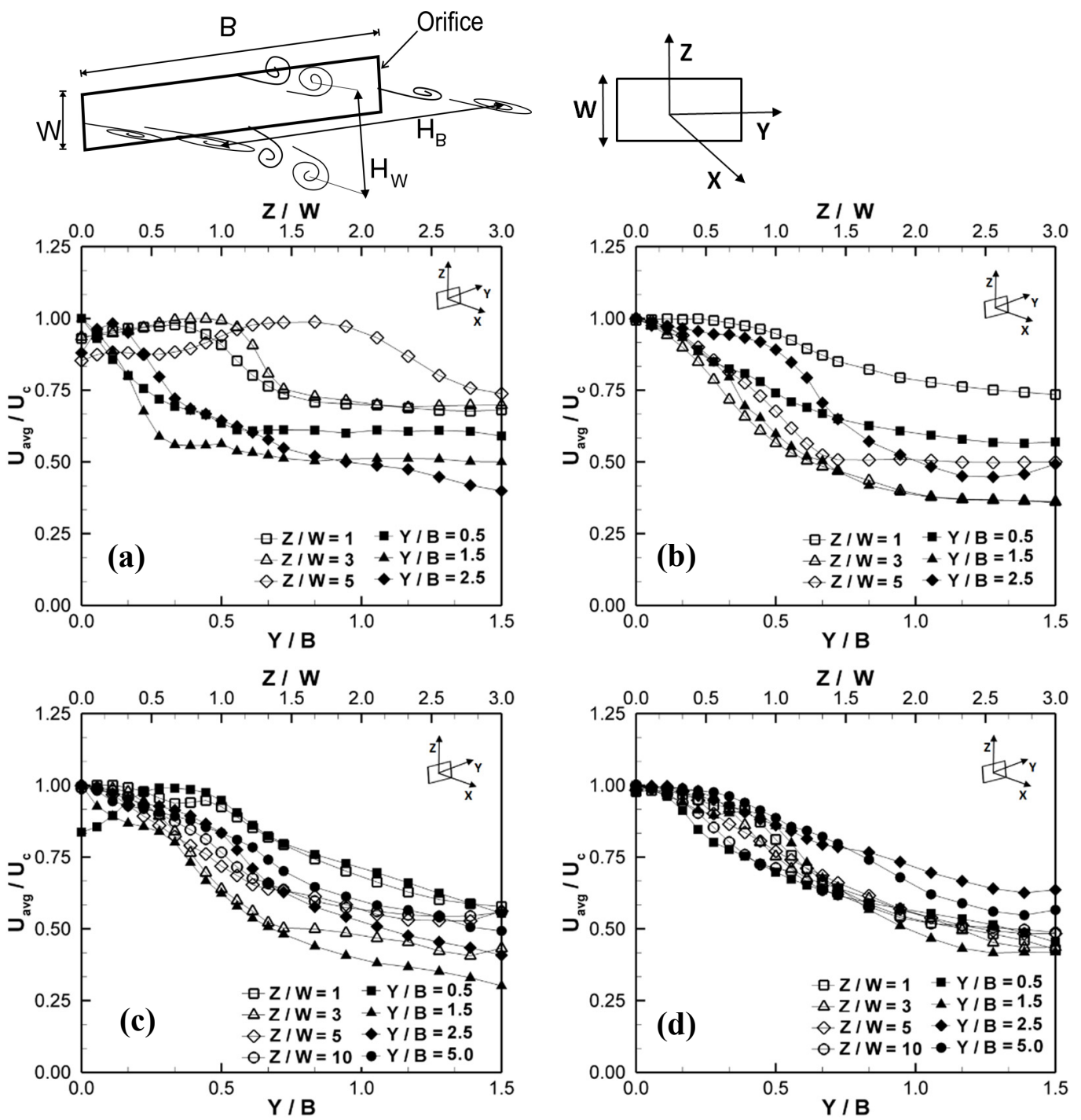

Figure 4.19: Time-averaged streamwise velocity profile at different downstream locations $(\mathrm{X} / \mathrm{W}=1,3,5$ and 10) in cross-stream directions $\mathrm{Y}$ and $\mathrm{Z}$ for rectangular orifice (AR2) at different actuation frequencies: (a) $1 \mathrm{~Hz}$, (b) $2 \mathrm{~Hz}$, (c) $4 \mathrm{~Hz}$ and (d) $6 \mathrm{~Hz}$. 
Table 4.6: Values of maximum streamwise velocity component at cross stream location $\left(U_{c}\right)$ in m/s for AR4 orifice

\begin{tabular}{|c|c|c|c|c|c|c|c|c|c|}
\hline \multirow{3}{*}{$x / W$} & \multicolumn{4}{|c|}{ XZ-plane } & f & \multicolumn{4}{|c|}{ XY-plane } \\
\hline & $1 \mathrm{~Hz}$ & $2 \mathrm{~Hz}$ & $4 \mathrm{~Hz}$ & $6 \mathrm{~Hz}$ & & $1 \mathrm{~Hz}$ & $2 \mathrm{~Hz}$ & $4 \mathrm{~Hz}$ & $6 \mathrm{~Hz}$ \\
\hline & & & & & $\mathbf{X} / \mathbf{W}$ & & & & \\
\hline 1 & 0.011 & 0.017 & 0.050 & 0.151 & 1 & 0.014 & 0.017 & 0.053 & 0.169 \\
\hline 3 & 0.024 & 0.026 & 0.086 & 0.142 & 3 & 0.022 & 0.025 & 0.083 & 0.142 \\
\hline 5 & 0.036 & 0.040 & 0.058 & 0.086 & 5 & 0.036 & 0.037 & 0.058 & 0.086 \\
\hline 10 & - & - & 0.038 & 0.051 & 10 & - & - & 0.038 & 0.051 \\
\hline
\end{tabular}


Figure 4.20: Time-averaged streamwise velocity profile at different downstream locations $(X / W=1,3,5$ and 10) in transverse directions $Y$ and $Z$ for rectangular orifice (AR4) at different actuation frequencies : (a) $1 \mathrm{~Hz}$, (b) $2 \mathrm{~Hz}$, (c) $4 \mathrm{~Hz}$ and (d) $6 \mathrm{~Hz}$. 


\subsection{Spectral analysis of synthetic jet}

The coherent structure is a connected large-scale turbulent fluid mass with a phasecorrelated vorticity having a size comparable to the transverse extent of the shear flow, which is responsible for significant transport of mass, heat and momentum (Hussain, 1983). The evolution of coherent structures, their propagation, interaction among themselves and with surrounding fluid and sustainability in downstream direction have a significant impact on local (space and time) or time-averaged behaviour. The nature of coherent structure depends on orifice geometry, fluid properties, Reynolds number, Strouhal number and nature of excitation. The objective of this section is to extract more information regarding the characteristics of coherent structure at various length scales in flow domain from the velocity signal. A study on the behaviour of coherent structures generated by synthetic jet in joint time-frequency domain provides more information compared to the velocity signal analysis in time domain or Fast Fourier transformation in frequency domain. The joint timefrequency $(\mathrm{TF})$ representation resolves the signal both in time and frequency domain, which helps to identify the localized events in time-frequency domain. The velocity signals acquired at different locations in downstream direction along the synthetic jet centerline is considered for the study of coherent structures in time, frequency and joint-time frequency domain in downstream direction. The velocity signals are acquired using S-type hot film. The four different orifices, a circular orifice of $10 \mathrm{~mm}$ diameter (D10), a square orifice of size $12 \times 12 \mathrm{~mm}^{2}$ (AR1), a rectangular orifice of aspect ratio two of size $9 \times 18 \mathrm{~mm}^{2}$ (AR2) and aspect ratio four of size $7.5 \times 30 \mathrm{~mm}^{2}$ (AR4) are selected to study the synthetic jet at 1 , 6 and $18 \mathrm{~Hz}$ actuation frequency. 



Figure 4.21: Flow visualization images of synthetic jet in XZ-plane (side view) for circular orifice (D10), square orifice (AR1), rectangular orifice of aspect ratio two (AR2) and for rectangular orifice of aspect ratio four (AR4) at ((a), (b), (c) and (d)) $1 \mathrm{~Hz}$ actuation frequency and ((i), (ii), (iii) and (iv)) at $6 \mathrm{~Hz}$ actuation frequency. (* The dots represent the location for S-type hot-film measurement for spectral analysis. It may be noted that images are not at same phase). 
The points considered for spectral analysis along the synthetic jet centerline are located at $\mathrm{X} / \mathrm{D}$ or $\mathrm{X} / \mathrm{W}=0,3,5$ and 8 at $1 \mathrm{~Hz}$ actuation frequency and at $\mathrm{X} / \mathrm{D}$ or $\mathrm{X} / \mathrm{W}=0$, 3, 8, 12 and 18 for 6 and $18 \mathrm{~Hz}$ actuation frequency for all four orifices. These locations are shown in Figure 4.21 as dots. The LIF images in XZ-plane for all four orifices at 1 and $6 \mathrm{~Hz}$ actuation frequency are shown in Figure 4.21. Due to limitation of laser power and lower limit of frame grabbing rate, the camera is not able to capture the images at $18 \mathrm{~Hz}$ actuation frequency due to low intensity of dye and higher rate of mixing and diffusion. The LIF images shown in Figure 4.21 are already discussed in the previous sections. Here, the images at different time instant are used for correlation with the time and frequency analysis.

\subsubsection{Velocity time trace}

The time trace of velocity signal represents the variation of velocity in time domain. Whenever a vortex ring crosses the measurement point, the magnitude of velocity increases and decreases with time that forms peaks in velocity signal. The number of peaks per second in signal is equal to the actuation frequency of synthetic jet. In downstream direction the presence of discrete peaks at actuation frequency in velocity time trace confirms the presence of large-scale coherent structures (in the form of vortex rings) produced by the synthetic jet. The non-dimensionalized velocity variation in time domain is shown in Figure 4.22 along the synthetic jet centerline for all the cases considered for spectral analysis. Due to directional insensitiveness of hot-film, the suction velocity of synthetic jet is sensed as positive velocity. This gives an additional peak between two consecutive ejection periods and doubles the number of peaks in velocity time trace near the orifice. The propagation of vortex ring in downstream direction is favoured only when the magnitude of velocity during ejection period is higher than that of suction period. Based on these assumptions the higher peaks are considered as ejection period peaks in velocity time trace shown in Figure 4.22. 
Except the velocity signal or time trace at 1 and $18 \mathrm{~Hz}$ actuation frequency for AR1 orifice case, the un-rectified signal shows occurrence of smaller peaks (suction period) at actuation frequency with magnitude less than $80 \%$ of the of higher peaks (ejection period).

The amplitude of suction effect at the center of orifice for circular orifice in Figure 4.22 (a) is comparatively much lower than the non-circular orifice as seen in Figure 4.22 (b), (c) and (d). For non-circular orifices (AR1, AR2 \& AR4), it is observed that the amplitude of suction stroke is nearly same as that of ejection stroke at the center of orifice with some difference in the area within the peaks. The complete vortex formation and its propagation is obtained only for AR1 among all three non-circular orifices at $1 \mathrm{~Hz}$ actuation frequency (see Figure 4.21 (b)). For AR2 orifice the vortex ring splits and there is a large expansion of vortex ring nearer to the orifice exit for AR4 (see Figure 4.21 (c)). The propagation of vortex ring for non-circular orifices is different from the circular orifice as the induced velocity of corners and/or shorter sides of vortex ring has higher velocity than the central region. Therefore, even though the amplitude of suction velocity is equal or at the same order of magnitude in comparison to ejection stroke, the vortex rings can be dragged out from the suction region by fast moving segments of vortex ring (see Figure 4.21(b)). The coherent structure at the length scale of orifice or the signature of synthetic jet pulsation in downstream direction is present till $\mathrm{X} / \mathrm{D}=8$ for $\mathrm{D} 10$ orifice and till $\mathrm{X} / \mathrm{W}=$ 5 for AR1 orifice (see Figure 4.22 (a) \& (b)). The pulsation in velocity time trace is lost immediately after the exit of the orifice for AR2 and AR4 orifice. At $6 \mathrm{~Hz}$ actuation frequency the signature of coherent structure at actuation frequency is present up to $\mathrm{X} / \mathrm{D}=$ 12 for $\mathrm{D} 10$, till $\mathrm{X} / \mathrm{W}=8$ for $\mathrm{AR} 1$ and $\mathrm{AR} 2$ orifices, and till $\mathrm{X} / \mathrm{W}=3$ for $\mathrm{AR} 4$ orifice (see Figure $4.22(\mathrm{e}-\mathrm{h})$. At $18 \mathrm{~Hz}$ actuation frequency, the coherent structures are present till $\mathrm{X} / \mathrm{D}=8$ for $\mathrm{D} 10$ orifice, till $\mathrm{X} / \mathrm{W}=8$ to $\mathrm{X} / \mathrm{W}=3$ for AR1 orifice and AR2 orifice. For AR4 
orifice pulsation in velocity signal is present until $\mathrm{X} / \mathrm{W}=3$ with an abrupt change in magnitude with time.
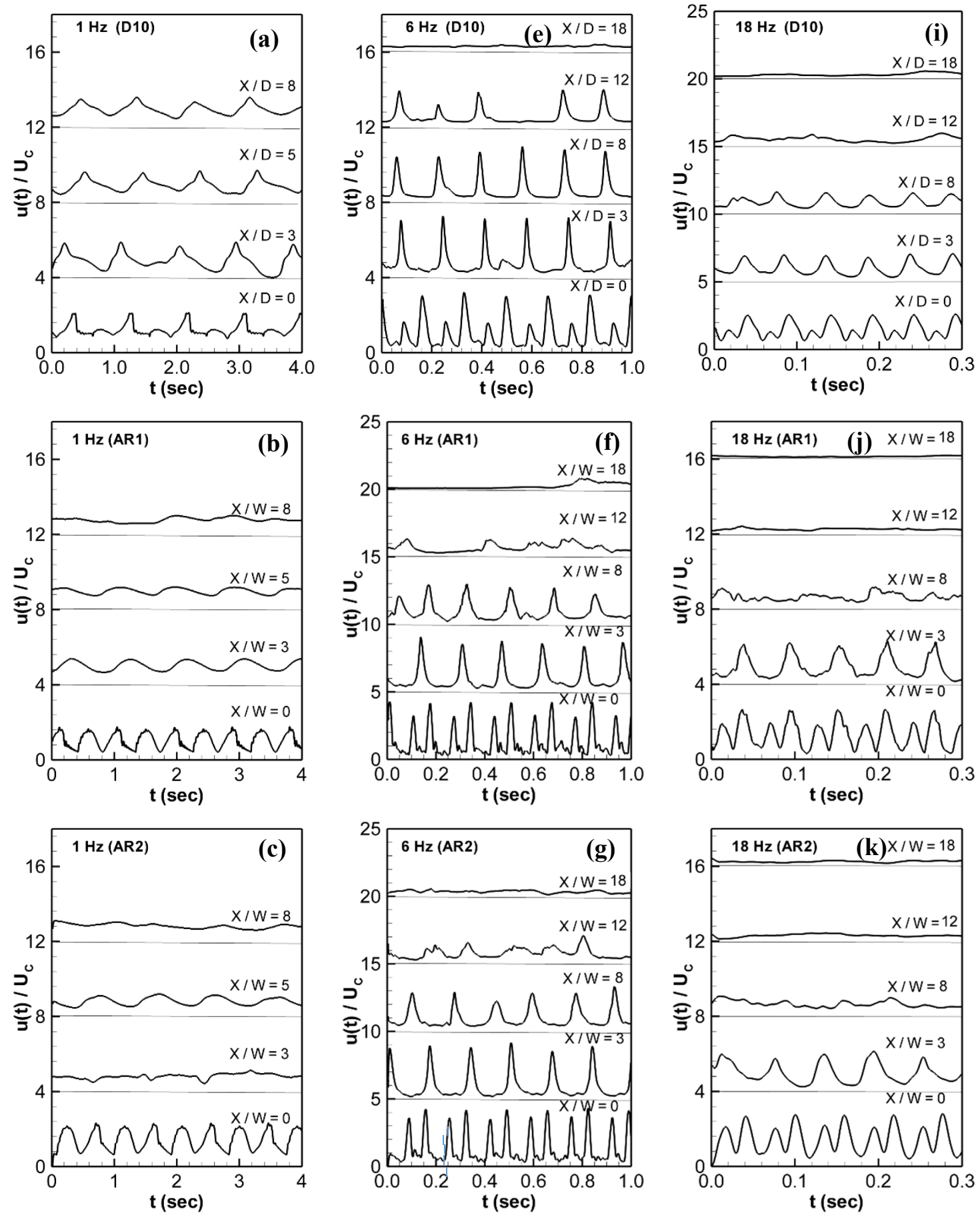

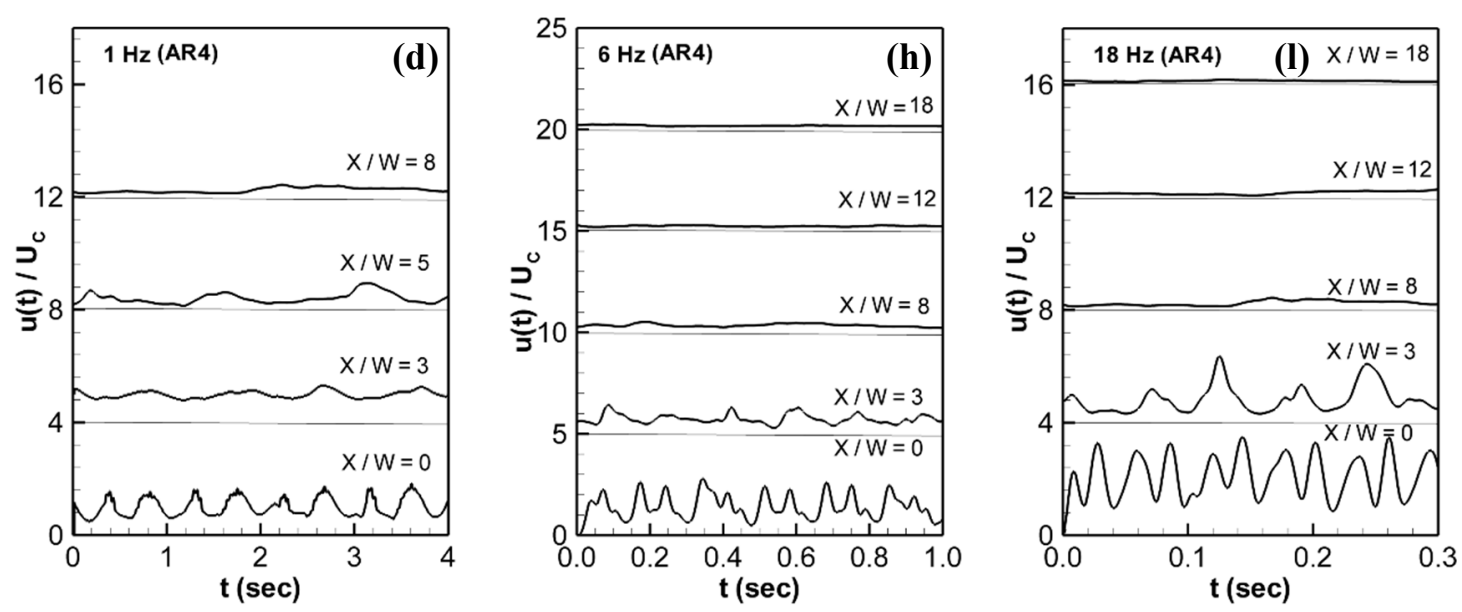

Figure 4.22: Velocity time trace of synthetic jet using hot-film along the synthetic jet centerline at different downstream locations for circular orifice (D10), square orifice (AR1), rectangular orifice of aspect ratio two (AR2) and rectangular orifice of aspect ratio 4 (AR4) at $1 \mathrm{~Hz}$ actuation frequency (a-d), $6 \mathrm{~Hz}$ actuation frequency (e-h) and $18 \mathrm{~Hz}$ actuation frequency (i-l). (* The plots are shifted upward by a step size of 4 or 5 for clarity).

It has been observed that for all four orifices, an increases in actuation frequency from 1 to $6 \mathrm{~Hz}$, increases the sustainability of large-scale coherent structure in downstream direction. Further increase in actuation frequency from 6 to $18 \mathrm{~Hz}$ reduces the propagation distance of large-scale coherent structure. At $1 \mathrm{~Hz}$ actuation frequency, since the induced velocity of vortex ring is low due to low $\mathrm{L} / \mathrm{D}_{\mathrm{h}}$ ratio and Reynolds number (see Table 4.3), the vortex rings form and are able to escape from the suction region. However, they bifurcate or break near the orifice exit as seen in Figure 4.21 (c) \& (d) for AR2 and AR4 orifice. For higher $\mathrm{L} / \mathrm{D}_{\mathrm{h}}$ ratio and Reynolds number, the vortex rings form and convect downstream but loses the pulsating nature at lower distance (see Figure 4.22 (a) (b)) in comparison to the $6 \mathrm{~Hz}$ actuation frequency as the vortex ring does not have enough energy to sustain their growing size and energy loss in downstream direction. At $18 \mathrm{~Hz}$ actuation frequency, an increase in $\mathrm{L} / \mathrm{D}_{\mathrm{h}}$ ratio and Reynolds number in comparison to $6 \mathrm{~Hz}$ actuation frequency for all the orifices causes an increase in turbulence level, strength of trailing jet and vortex interaction as observed in Figure 4.21 (i - iv). Correspondingly, a greater loss of coherency is observed in velocity time trace in Figure 4.22 (i - 1). In spite of all these reasons, the effect of orifice corner and variation in induced velocity of different segments 
of vortex rings controls the presence of coherent structure. This effect is more profound for AR4 orifice where the vortex rings break quickly leaving no signature of pulsation after $\mathrm{X} / \mathrm{W}=0$ even at higher $\mathrm{L} / \mathrm{D}_{\mathrm{h}}$ ratio and Reynolds number (see Figure $4.22(\mathrm{~h}) \&(1)$ ).

\subsubsection{Power spectral density}

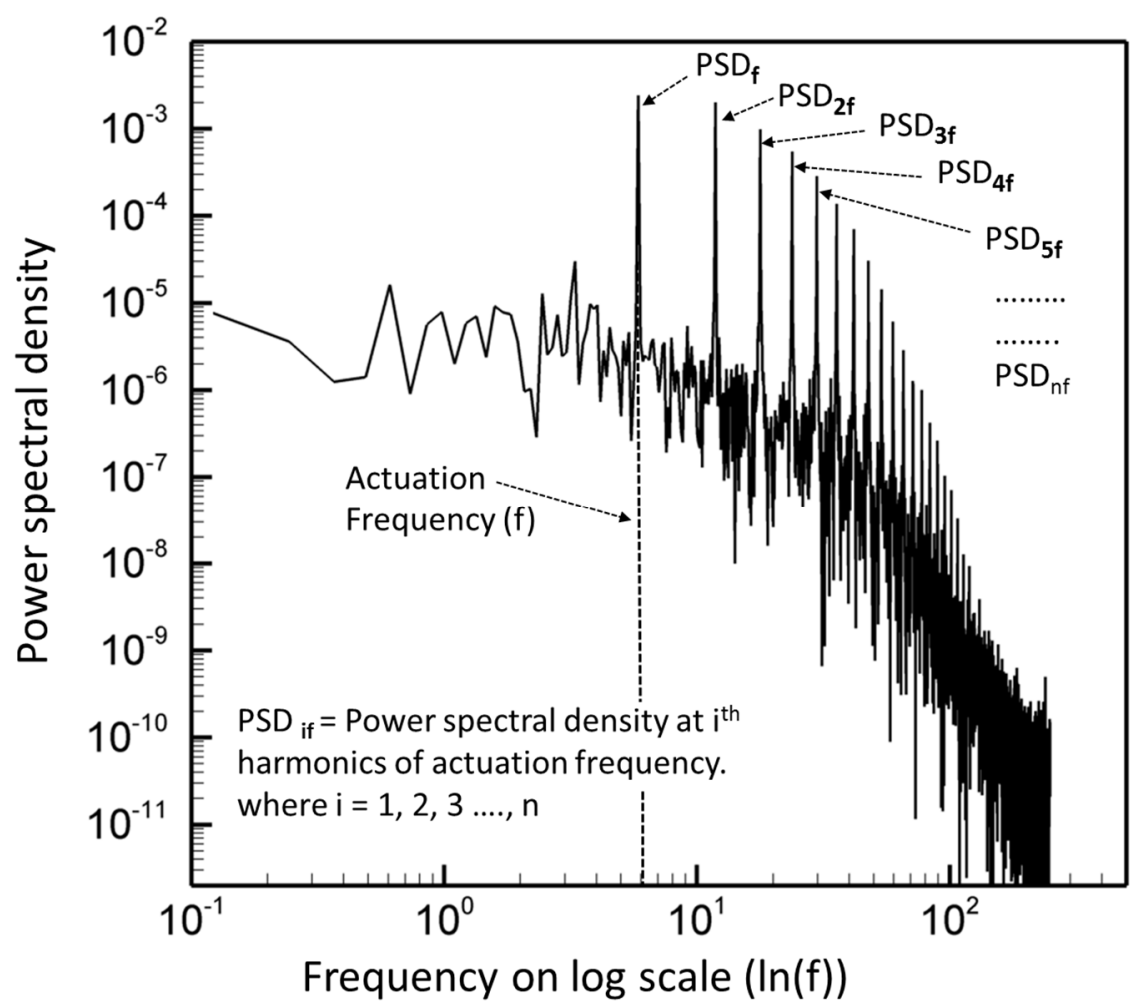

Figure 4.23: Power spectral density (PSD) distribution along the centerline at $6 \mathrm{~Hz}$ actuation frequency for $10 \mathrm{~mm}$ circular orifice (D10) at $\mathrm{X} / \mathrm{D}=3$.

This section reports the frequency content of velocity-time trace discussed in previous section. Figure 4.23 shows a typical power spectral density distribution of velocity-time trace of circular orifice (D10). The small-scale coherent structures at integer multiple of actuation frequency are known as harmonics of actuation frequency. The power spectral density (PSD) of velocity signal calculated using Fast Fourier transformation with windowing and averaging gives the power distribution of signal per unit frequency i.e. energy of the velocity time trace distributed in frequency domain. The power spectral density of a velocity signal is useful to know the distribution of energy in frequency domain 
where the total energy associated with the velocity signal is distributed in frequency domain. In power spectral density distribution, the presence of coherent structures in synthetic jet can be verified from the peaks occurring at the harmonics of actuation frequency. The existence of peak at first harmonic (actuation frequency) in spectral density distribution indicates that the coherent structure of orifice length scale (largest vortex ring) is present at that location. The others peaks which are located at integer multiple of actuation frequency represent the coherent structures of smaller length scale. In case of synthetic jet, the magnitude of peak at different harmonics decreases with increasing order of harmonic frequency (Debiasi et al., 2004). It means that the highest energy is associated with the coherent structure at first harmonic, then second harmonic and so on. The location of actuation frequency (f) is shown by the vertical dotted line marked with symbol ' $\mathrm{f}$ ' in Figure 4.24 .
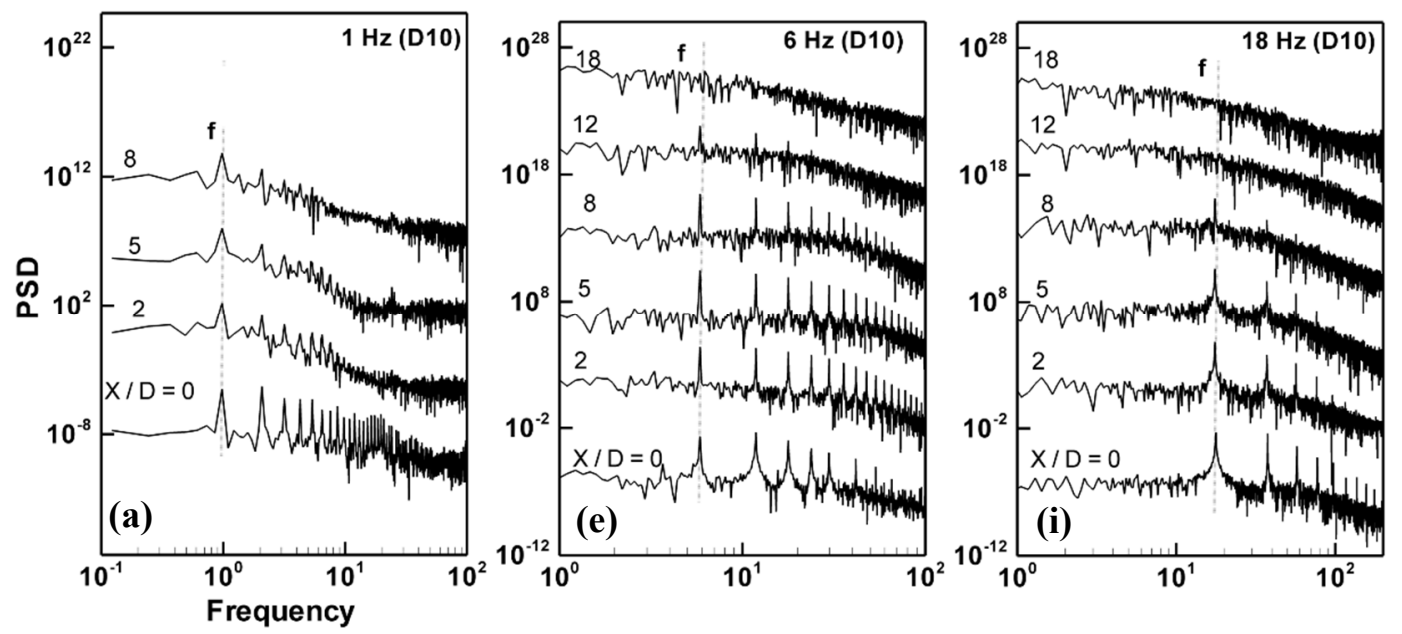



Figure 4.24: Power spectral density (PSD) of streamwise velocity along synthetic jet centerline at different downstream locations for circular orifice (D10), square orifice (AR1), rectangular orifice (AR2) and rectangular orifice (AR4) at (a-d) $1 \mathrm{~Hz}$, (e-h) $6 \mathrm{~Hz}$ and (i-l) $18 \mathrm{~Hz}$ actuation frequency.

As stated earlier, the number of peaks is doubled due directional insensitiveness of hot-film (see Figure 4.22 at $\mathrm{X} / \mathrm{W}$ or $\mathrm{X} / \mathrm{D}=0$ ). This also increases the amplitude of second harmonics compared to the first harmonic at $\mathrm{X} / \mathrm{D}$ or $\mathrm{X} / \mathrm{W}=0$ as seen in Figure 4.24 at all 
cases. This effect is more profound at $1 \mathrm{~Hz}$ actuation frequency for non-circular orifices as seen in Figure 4.24 (b), (c) and (d) at $\mathrm{X} / \mathrm{W}=0$. The sharp peaks present till $\mathrm{X} / \mathrm{D}=8$ for D10 orifice and flattened peaks till X/W $=5$ for AR1 orifice in Figure 4.24 (a) and (b) closely support the presence of large scale coherent structure as shown in Figure 4.21 (a) and (b). The peaks are not present in downstream direction after X/W $=0$ for AR2 and AR4 orifices at $1 \mathrm{~Hz}$ actuation frequency in Figure 4.24 (c) and (d). This is connected to no distinct formation of vortex rings in LIF images as shown in Figure 4.21 (c) and (d). No recognizable peak is present at $\mathrm{X} / \mathrm{W}=2$ and beyond for $\mathrm{AR} 1, \mathrm{AR} 2$ and $\mathrm{AR} 4$ orifices except at first harmonics frequency. At $6 \mathrm{~Hz}$ actuation frequency, the peak at first and second harmonic frequency is present till $\mathrm{X} / \mathrm{D}=12$ for $\mathrm{D} 10$ orifice, till $\mathrm{X} / \mathrm{W}=5$ for $\mathrm{AR} 1$ orifice, till $\mathrm{X} / \mathrm{W}=8$ for $\mathrm{AR} 2$ orifice and till $\mathrm{X} / \mathrm{W}=2$ for AR4 orifice as shown in Figure $4.24(\mathrm{e}$ h). When the actuation frequency is increased further to $18 \mathrm{~Hz}$, the presence of peak at first and second harmonic frequency decreases to $\mathrm{X} / \mathrm{D}=5$ for $\mathrm{D} 10$ orifice, to $\mathrm{X} / \mathrm{W}=5$ for $\mathrm{AR} 1$ and AR4 orifice as compared to $6 \mathrm{~Hz}$ actuation frequency.

Irrespective of the actuation frequency in Figure 4.24, peaks at higher order harmonics are present for circular orifices D10 up to a longer distance in comparison to non-circular orifices AR1, AR2, and AR4. The peaks disappear within a very short distance $(\mathrm{X} / \mathrm{W}<5)$ for AR4 orifice in comparison to AR1 and AR2 orifice, which is supported by the velocity signal shown in Figure 4.22. The other observation is that the peak at the first harmonic frequency disappears at the end in comparison to the peaks at higher order of harmonics. Since the peaks in power spectral density distribution at multiple of actuation frequency represents the coherent structure of different length scales associated with the synthetic jet, their absence indicates a non-pulsatile flow. Therefore, if the peak at first harmonic frequency disappears in power spectral density, it indicates that there is no coherent structure present at any order of harmonic frequency. A plot showing the 
magnitude of peaks present at first harmonic frequency in power spectral density distribution with respect to the downstream distance from the orifice is shown in Figure 4.25 , which provides an overview of coherent structure present in synthetic jet as reported in the next section.
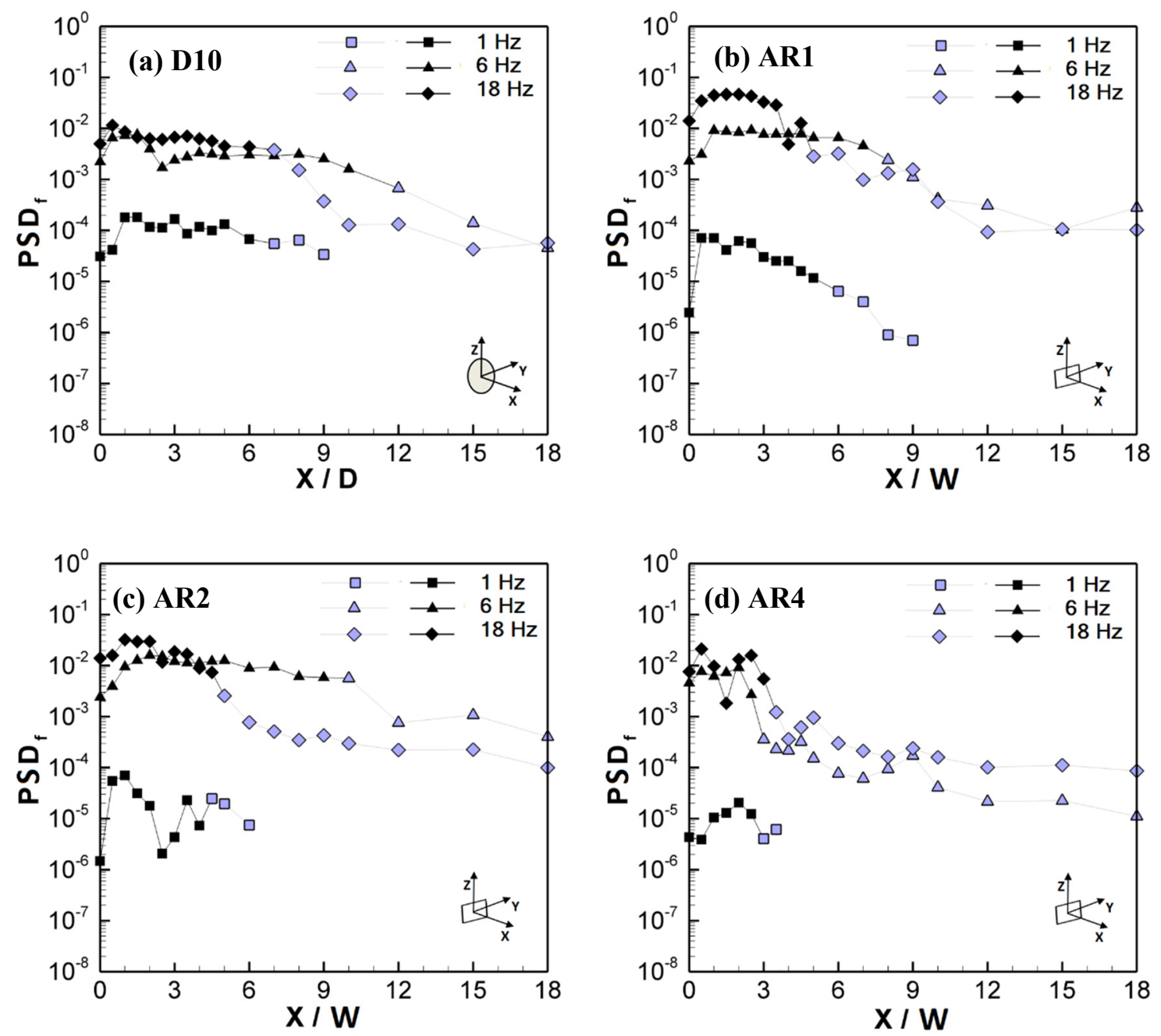

Figure 4.25: Magnitude of power spectral density at first harmonic along the synthetic jet centerline for (a) D10, (b) AR1, (c) AR2 and (d) AR4 orifices at 1, 6 and $18 \mathrm{~Hz}$ actuation. (*The values of PSDf more than $10 \%$ of maximum achieved value at first harmonic are represented by dark symbols and the value less than $10 \%$ are represented by shaded symbols.)

The variation in the magnitude of power spectral density at first harmonic frequency or in other words energy associated with coherent structure at actuation frequency is shown in Figure 4.25. The magnitude of peak at first harmonic less than $10 \%$ (above the error zone $\pm 5 \%)$ of maximum value achieved after suction zone $(\mathrm{X} / \mathrm{D}$ or $\mathrm{X} / \mathrm{W}<2$ to 3$)$ is considered 
as threshold for signature analysis of large-scale coherent structure at orifice length scale (vortex ring) in far downstream distance. For most cases the discrete peaks in velocity time trace (Figure 4.22) also disappears within the same region between $\mathrm{X} / \mathrm{D}=9$ to 12 as observed in Figure 4.25. Another observation is that at $6 \mathrm{~Hz}$ actuation frequency the signature of coherent structure at first harmonic is longer than that at 1 and $18 \mathrm{~Hz}$ actuation frequency for all four orifices seen in Figure 4.25 by the presence of peaks. In comparison to all four orifices, the loss of coherency occurs very rapidly (before $\mathrm{X} / \mathrm{W}=3$ ) for AR4 orifice at all three actuation frequencies due early vortex breakdown as seen in Figure 4.21.

\subsubsection{Joint time-frequency analysis}

The Fast Fourier transformation (FFT) of the signal represents spectral information of a fluctuating signal with no temporal information. Therefore, an event localized in time is masked due to background averaged spectral content. The time trace representation provides the temporal information but no spectral information. Signals are often nonstationary and complex in nature. Analysis of these signals can be simplified if joint timefrequency representation (TF) of signal is used. To have both temporal as well as spectral information, the joint time-frequency domain analysis is useful. The joint time-frequency (TF) representation resolves a signal (function of time) in both time and frequency domain. In joint time-frequency representation the spectral contribution at any particular time instant can be obtained by drawing a thin time strip parallel to the frequency axis and similarly the frequency range at particular instant of time can be obtained by drawing a line parallel to the time axis. The wavelet transformation is a well-accepted and a powerful tool for transforming a real time domain signal into joint time-frequency domain. The wavelet transformation technique has been applied in various applications like quantum physics (Sadiku et al., 2005). The other applications are identification and characterization of transient random processes in wind flow in geophysical phenomenon, in earthquake and 
ocean engineering (Kareem \& Gurley, 1999). The wavelet transformation is also useful in medical science for blood pressure waveform analysis (Melis et al., 2009) and ECG characterization (Sahambi \& Bhatt, 1997). It is also applied for musical transcription (Wakefield \& Pielemeier, 1992), in speech reorganization, in communication for signal processing and controlling in the general field of acoustics. In fluid dynamics, the wavelet transformation is used for the study of the transient signals propagation in inhomogeneous media (Ginette et al., 1998). Analysis of coherent structures present in near wall of turbulent channel flow obtained through numerical simulation has been studied by Farge et al. (1990). This technique is also applied in the detection of vortex ring generated from PIV images in an impulsively starting jet (Schram and Riethmuller, 2001). The length scales identification in synthetic jet for which energy fluctuations and intermittency are important is identified with the help of wavelet transformation by Mallinson et al. (2004). Narasimha (2007) used the wavelet transformation in detection of coherent structures for jets and plumes at different length scales using laser-induced fluorescence (LIF) and dye-flow visualization technique.

In wavelet transformation, a small wave (prototype for generating the window functions) known as mother wavelet is scaled in amplitude and translated in time to fit within the portion of velocity signal inside the window size. The spectral component is computed and then window is shifted on the signal to compute the spectral component for every position. This process is repeated many times over the entire signal by reducing the window size until all the possible frequency can be resolved. Finally, a time-frequency representation of signal is obtained with different resolutions (Farge M., 1992). In any practical signal, the event occurring at lower frequency exists for longer duration that requires low resolution in time domain but high resolution in frequency domain. Whereas the event, which occurs at higher frequency, exists for short duration and to detect them, 
higher resolution in time domain with lower resolution in frequency domain is required. The wavelet transformation meets this requirement and this is the key feature of wavelet analysis, which makes it suitable for analysing most of the practical signal in time frequency domain. The general definition (Sahambi et al., 1997) of wavelet equation is:

$\psi_{a}(t)=\frac{1}{\sqrt{a}} \psi(t / a)$

Where $\psi_{a}(t)$ is the dilation of $\psi(t)$ by the scale factor ' $a$ '. To conserve the energy during the transformation and to facilitate the reconstruction of original signal from the transformed wavelet, the factor $1 / \sqrt{a}$ is used for energy normalization in above equation. The wavelet transform of a signal $u(t)$ at scale factor ' $\mathrm{a}$ ' and position ' $\tau$ ' is carried out as: $W u(a, \tau)=\frac{1}{\sqrt{a}} \int_{-\infty}^{\infty} u(t) \psi^{*}\left(\frac{t-\tau}{a}\right) d t$

Depending on the magnitude of scale factor, the mother wavelet is contracted or expanded over the signal in time domain. The smaller value of scale factor provides finer detail of signal in time domain and approaches towards the global value with increase in its value. For wavelet transformation, Choi-Williams distribution method is applied due to its potentially better performance for impulsive or sinusoids with quasi-constant frequency signals (Hlawatsch \& Manickam, 1995). The Choi-Williams distribution is a Cohen-class distribution representing energy distribution in joint time-frequency domain. In ChoiWilliams distribution proposed by Choi \& Williams (1989), where the cross terms present in Winger-valley distribution (see Figure 4.26 (a) \& (b)) are suppressed by exponential kernel. A bilinear time-frequency distribution (here wavelet transformation) possesses a spurious terms (here peaks) for multicomponent signal. This spurious term is called as cross terms. However, the drawback is occurrence of negative value while suppressing the crossterms and therefore a proper care should be taken while deciding the cross term suppression 
co-efficient $(\alpha)$ (Hlawatsch \& Manickam, 1995). In Choi-William wavelet transformation the cross-term suppression coefficient $(\alpha)$ suppresses the cross term but there is an adverse effect on time-frequency resolution. Therefore, different cross-term suppression coefficient is used for each wavelet transformation such that the negative magnitude does not occur and maximum possible resolution is achieved. A code using advanced signal processing toolkit is written on LabVIEW platform for wavelet transformation to resolve the velocity signal in joint time-frequency domain at a particular point. The energy obtained from wavelet transformation is normalized by square of maximum averaged velocity $U_{c}$.

The joint time frequency representation, Fast Fourier Transformation and corresponding velocity signal in time domain is shown in Figure 4.26 (c) for D10 orifice at $6 \mathrm{~Hz}$ actuation frequency. The energy distribution at different harmonic of actuation frequency is extracted from the wavelet transformation and presented in Figure 4.26 (d). The energy of initial harmonics that represent the large-scale coherent structures contains the major portion of the total energy while the higher harmonics have lower percentage of total energy. A band parallel to time axis and with peaks at the rate of actuation frequency (see Figure 4.26 (e)) in time-frequency representation represents a continuous wave superimposed with periodic pulses (vortex rings ) in time domain. In time-frequency representation, the signature of synthetic jet is seen as the energy ribs parallel to the frequency axis combined with various waves in the form of band parallel to the time axis at the multiple of harmonic frequency. The small-scale coherent structure that occurs at higher frequency for small time can be detected through proper time step $(\Delta \mathrm{t})$, frequency resolution $(\Delta f)$ and cross term suppression coefficient $(\alpha)$. 


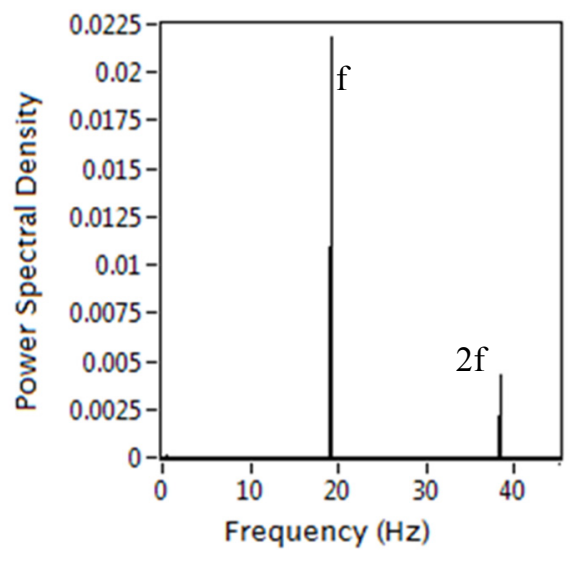

(a) Fast Fourier transformation

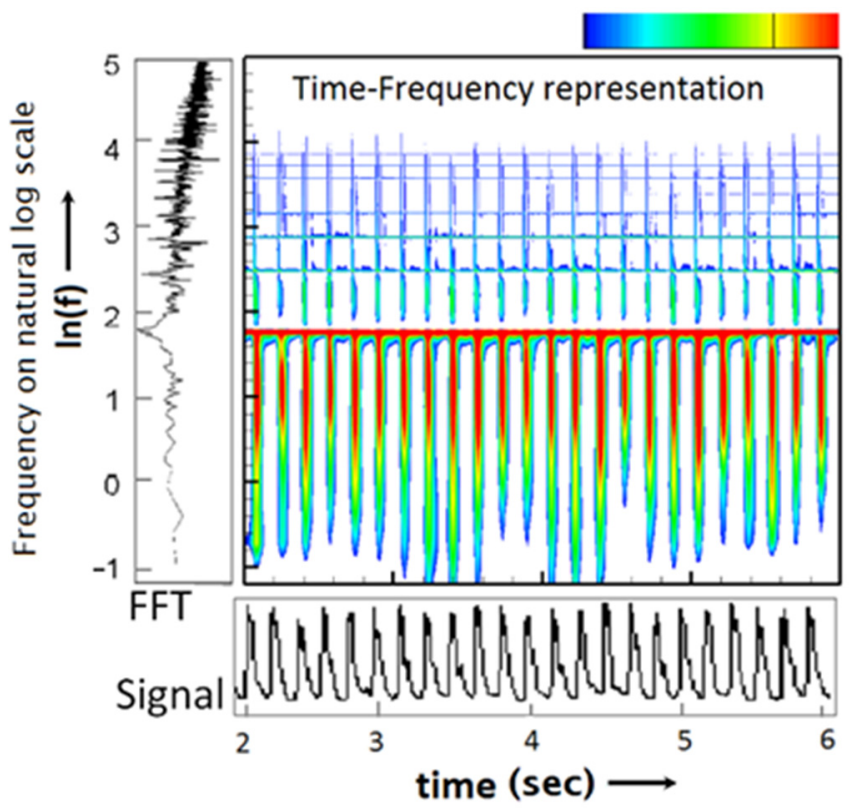

(c) Choi-Williams distribution

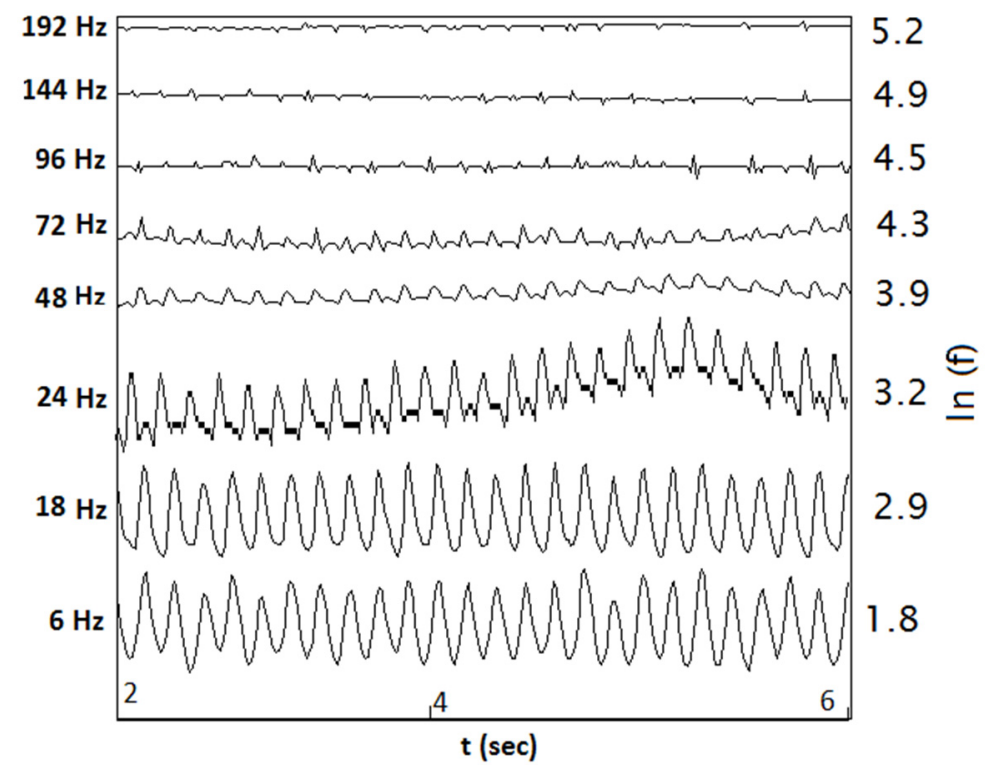

(b) Winger-Valley distribution

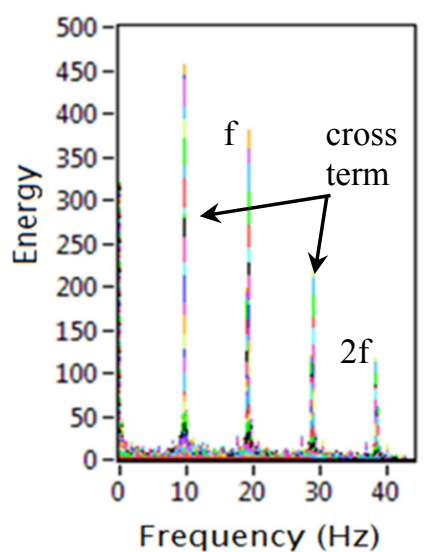






(e) Joint-time frequency representation

Figure 4.26 (a) Fast Fourier transformation and (b) Winger-Valley distribution of velocity signal at $18 \mathrm{~Hz}$ actuation frequency orifice at $X / D=5$. (c) Representation of velocity signal at $6 \mathrm{~Hz}$ actuation frequency at $X / D=5$ in time, frequency and joint time-frequency domain with (d) extracted value of energy form Time-Frequency representation plot at integer multiple of actuating frequency or at harmonic frequency. (e) 3-Dimendional view of joint time frequency representation of AR1 orifice for $6 \mathrm{~Hz}$ actuation frequency at $\mathrm{X} / \mathrm{D}=3$.

The structure present in the Choi-William transformation of velocity signal is the representation of energy density in time-frequency domain. Farge (1992) reported different types of flow structures and their corresponding representation in joint time-frequency domain. They are: (a) If the flow field is superposition of waves, the energy density is distributed in phase space as bands (parallel to frequency axis) and each band corresponds to an excited wave number, (b) if the flow field is a set of localized structure or coherent structure, cone like patterns pointing to an excited frequency occurs, (c) if the flow field is superposition of wave packets, energy density shows like patches and (d) if the flow field is some kind of noise the energy density is randomly distributed without any pattern. The nature of fluid flow at the measurement point can be obtained from the type of structure present in the wavelet transformation.

The wavelet transformation results for D10 and AR1 orifices at $1 \mathrm{~Hz}$ actuation frequency have been compared in Figure 4.27. A vortex ring appears in the form of ribs parallel to the frequency axis with decreasing apex height in frequency domain. The vortex 
rings which are laminar, well separated with absence or presence of trailing jet (see Figure 4.21 (b), represent themselves in the form of ribs which are distinguishable from each other and well separated on time axis as seen in Figure 4.27 (i). In Figure 4.27 (iii), the synthetic jet lost its signature before $\mathrm{X} / \mathrm{W}=8$, because the number of ribs are not periodic and less than the number of ribs in comparison to actuation frequency. If the trailing jet is isolated and in the form of smaller vortex ring (see Figure 4.21 (a)), the wavelet transformation shows small conical structures at second harmonics (2f) in Figure 4.27 (a). The ribs parallel to time axis is continuous at second harmonics location indicating that some amount of vorticity is always present at the measurement point. Till $\mathrm{X} / \mathrm{D}=8$, leading vortex ring or large scale coherent structures are present with significant magnitude but the trace of trailing jet vortex rings disappears after $\mathrm{X} / \mathrm{D}=5$ at second harmonics.

Figure 4.28 compares the wavelet transformation results of AR2 and AR4 orifice at $1 \mathrm{~Hz}$ actuation frequency. The periodic distribution of conical shape structures localized in time domain are present at first harmonic with small trace at second harmonic in Figure 4.28 (a) at $\mathrm{X} / \mathrm{W}=3$. The splitting of ribs into several patches in time domain at downstream locations $\mathrm{X} / \mathrm{W}=5$ and 8 in Figure 4.28 (b) and (c) for AR2 orifice diameter at $1 \mathrm{~Hz}$ actuation frequency can be correlated to stretching and tearing of vortex ring in Figure 4.21 (c). The energy density distribution is random with some coherent structures present at $\mathrm{X} / \mathrm{W}$ $=8$. There is no vortex formation for AR4 orifice at $1 \mathrm{~Hz}$ actuation frequency (see Figure 4.21 (d)). This leads to random coherent structures of different energy scale at $\mathrm{X} / \mathrm{W}=3$ and 5 in Figure 4.28 (i) and (ii).The splitting of larger strips into thinner streaks in time domain towards higher frequency in Figure 4.28 (iii) represents the broad spectrum of vortices in flow field.

Figure 4.29 compares the wavelet transformation results of D10 and AR1 orifice at $6 \mathrm{~Hz}$ actuation frequency. There are coherent structures superposed over the rib of 
continuous vorticity as sharp peaks. These peaks are localized in both time and frequency domain for D10 and AR1 orifice at $6 \mathrm{~Hz}$ actuation frequency in Figure 4.29 (a) and (i) respectively at $\mathrm{X} / \mathrm{D}=3$. The maximum share of energy are observed at first harmonics and its multiple for $\mathrm{D} 10$ orifice at $\mathrm{X} / \mathrm{D}=3,5$, and 8 . The maximum contribution for AR1 orifice is seen at first harmonics. The closely spaced fine streaks in time domain after the second harmonic in Figure 4.29 (ii) indicates the presence of small scale coherent structure at higher frequency and these structures are well mixed in time as seen in Figure 4.29 (iii) at $\mathrm{X} / \mathrm{W}=12$. Figure 4.30 compares the wavelet transformation results of AR2 and AR4 orifice at $6 \mathrm{~Hz}$ actuation frequency. The pattern of wavelet analysis for AR2 orifice in Figure 4.30 is similar to the pattern seen Figure 4.29 for AR1 orifice. However some nonperiodic coherent structure are present at lower frequency at $\mathrm{X} / \mathrm{W}=12$ in Figure 4.30 (c). The Figure 4.30 (i-iii) for AR4 orifice shows patches instead of ribs as no well separated vortex rings are seen Figure 4.21 (iv).

The signature of large-scale coherent structure at higher order of harmonics in the wavelet transformation shown in Figure 4.31 and Figure 4.32 at $18 \mathrm{~Hz}$ actuation frequency decreases drastically in comparison to the wavelet analysis shown in Figure 4.29 and Figure 4.30 (c) at $6 \mathrm{~Hz}$ actuation frequency. The signature of periodic actuation is visible in the form of small peaks over the continuous band at first harmonic in Figure 4.31 ((a), (i)) and Figure 4.32 ((a), (i)). The energy level at first harmonic decreases rapidly to a very small value after $\mathrm{X} / \mathrm{D}$ or $\mathrm{X} / \mathrm{W}=3$ and finally randomly distributed patches are seen at $\mathrm{X} / \mathrm{D}=12$ in Figure 4.31 (b) and (c) for D10 orifices. For other three non-circular orifices AR1, AR2 and AR4, the signature of large-scale coherence structure or band of vorticity are present at $\mathrm{X} / \mathrm{W}=3$ at first harmonics. 

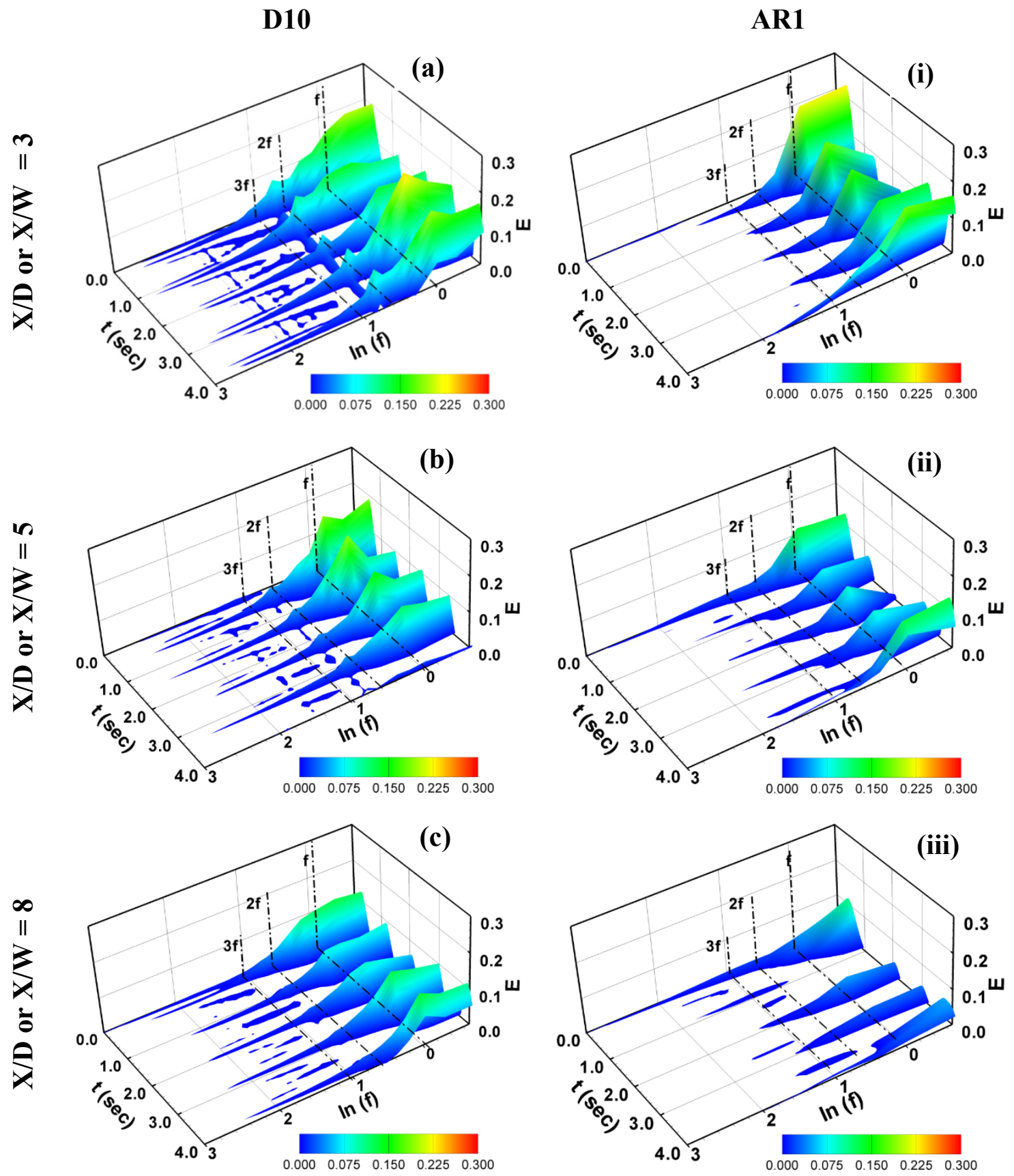

Figure 4.27: Choi-William transformation at $1 \mathrm{~Hz}$ actuation frequency in downstream direction along the synthetic jet centerline at three different stream wise locations $X / D$ or $X / W=3,5$ and 8 for circular orifice (D10) are shown in ((a), (b) \& (c))and for square orifice(AR1) are shown in ((i), (ii) \& (iii)). 

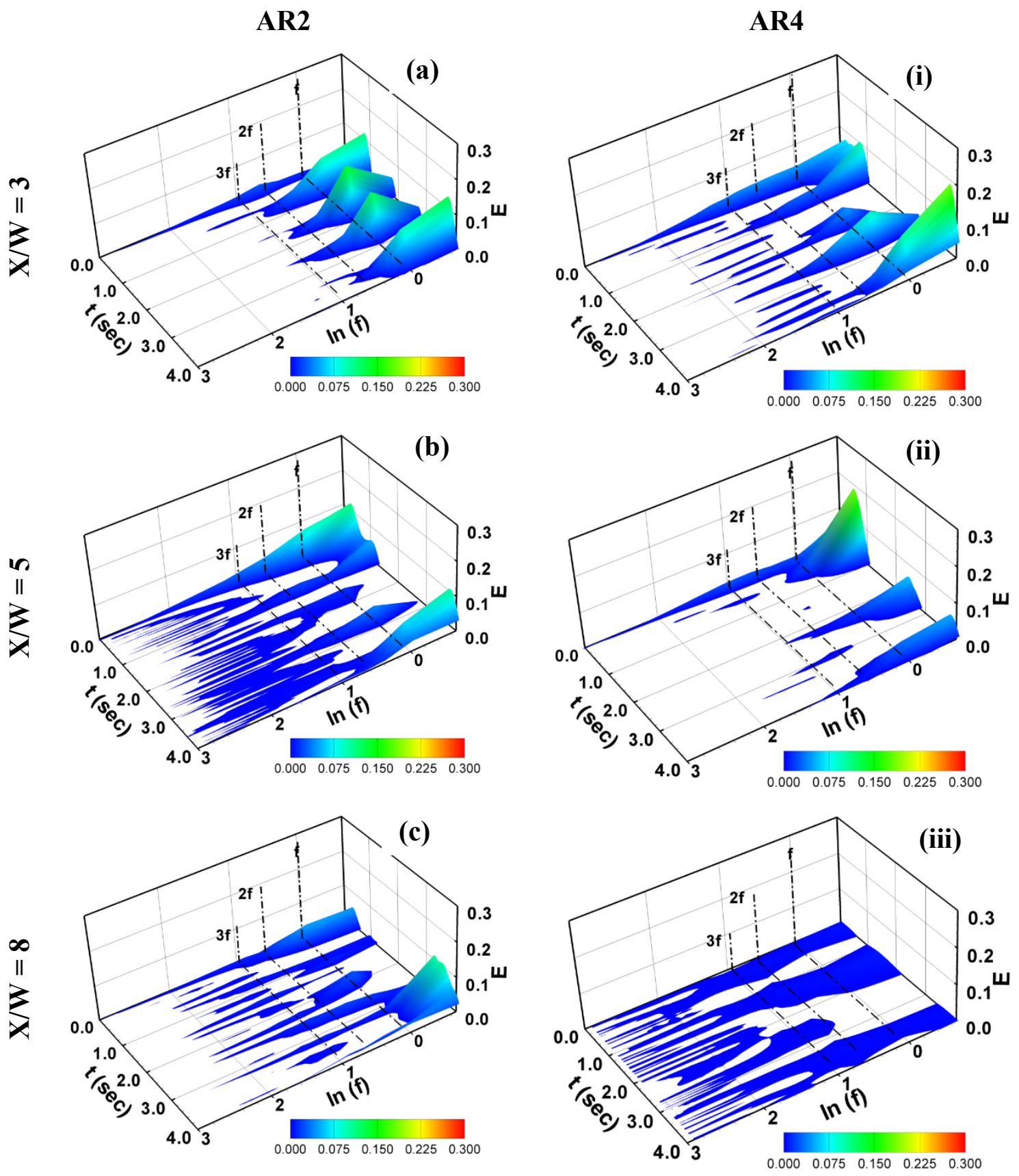

Figure 4.28: Choi-William transformation at $1 \mathrm{~Hz}$ actuation frequency in downstream direction along the synthetic jet centerline at three different stream wise locations $X / W=3,5$ and 8 for rectangular orifice (AR2) are shown in ((a), (b) \& (c))and for rectangular orifice(AR4) are shown in ((i), (ii) \& (iii)). 
D10
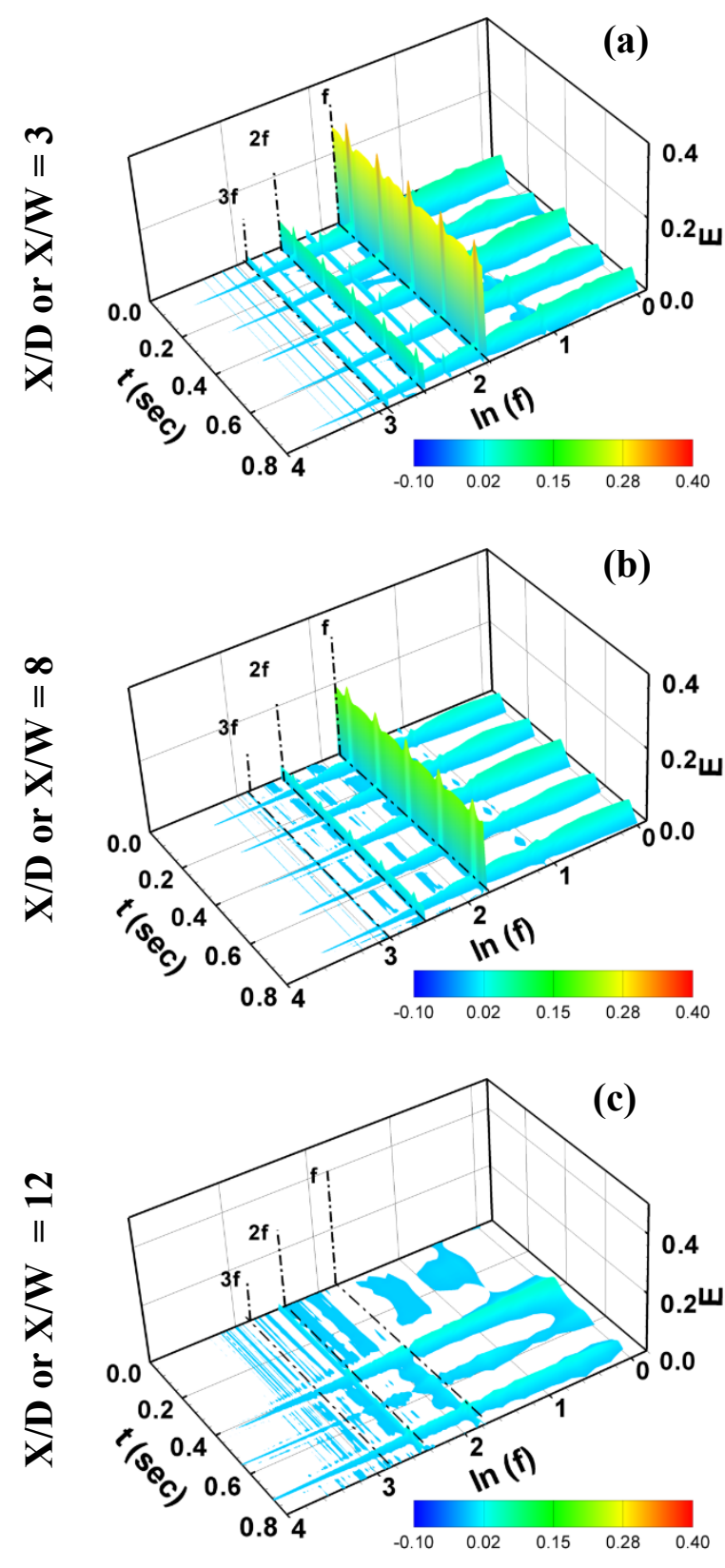

AR1


Figure 4.29: Choi-William transformation at $6 \mathrm{~Hz}$ actuation frequency in downstream direction along the synthetic jet centerline at three different stream wise locations $X / D$ or $X / W=3,8$ and 12 for circular orifice (D10) are shown in ((a), (b) \& (c))and for square orifice(AR1) are shown in ((i), (ii) \& (iii)). 

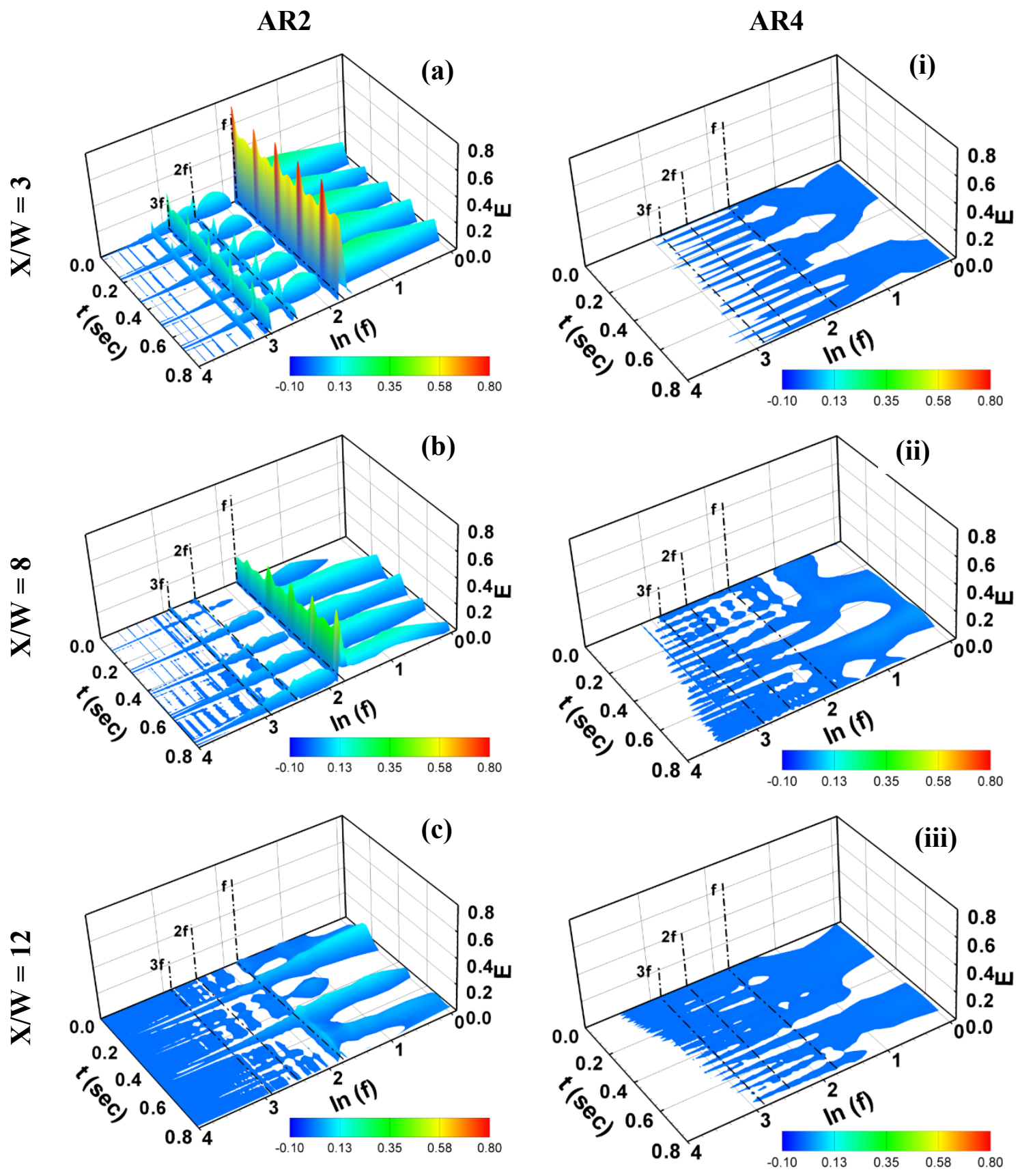

Figure 4.30: Choi-William transformation at $6 \mathrm{~Hz}$ actuation frequency in downstream direction along the synthetic jet centerline at three different stream wise locations $X / W=3,8$ and 12 for rectangular orifice $(A R 2)$ are shown in $((a),(b) \&(c))$ and for rectangular orifice(AR4) are shown in ((i), (ii) \& (iii)). 
D10

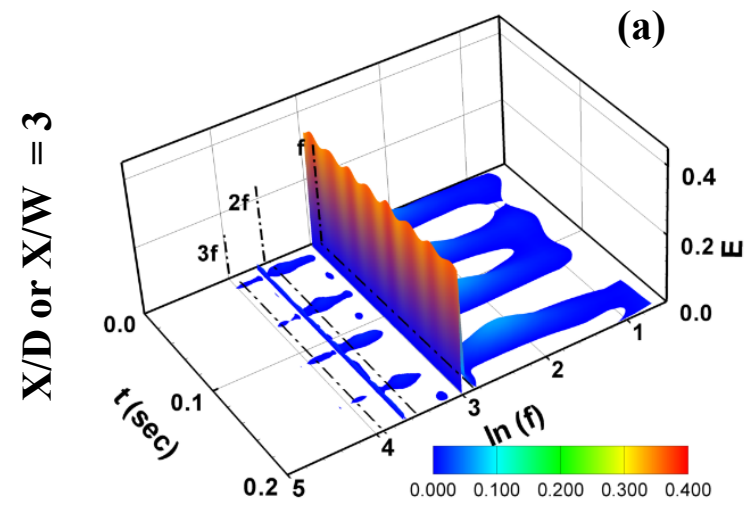

(b)

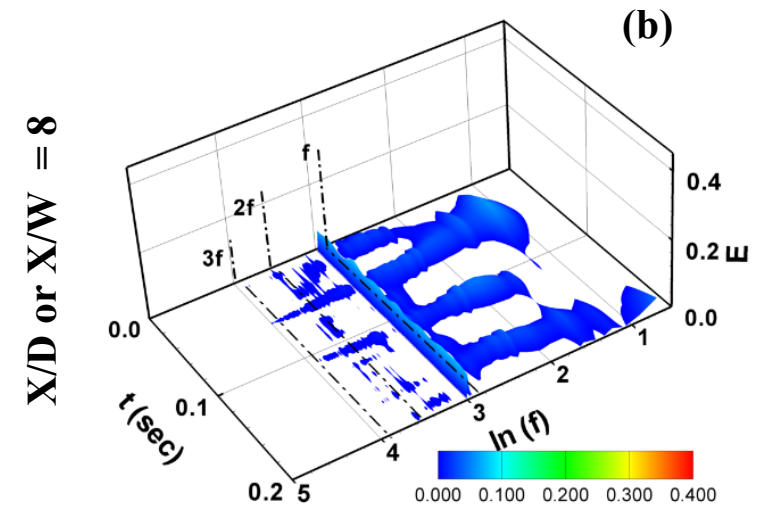

(c)

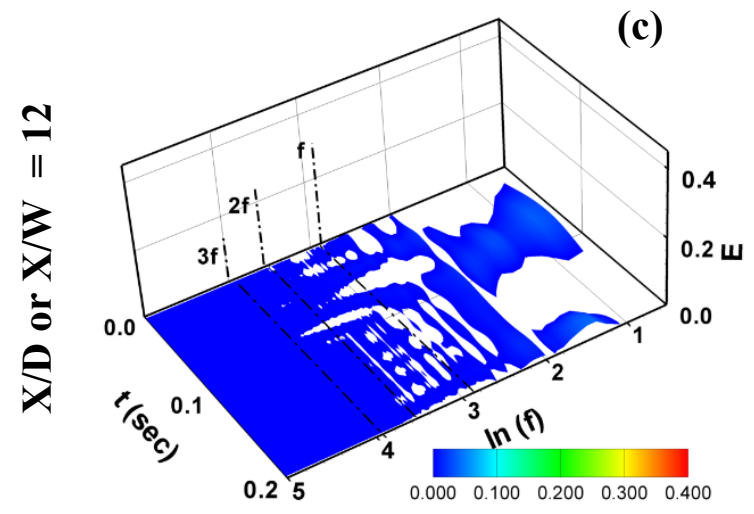

AR1

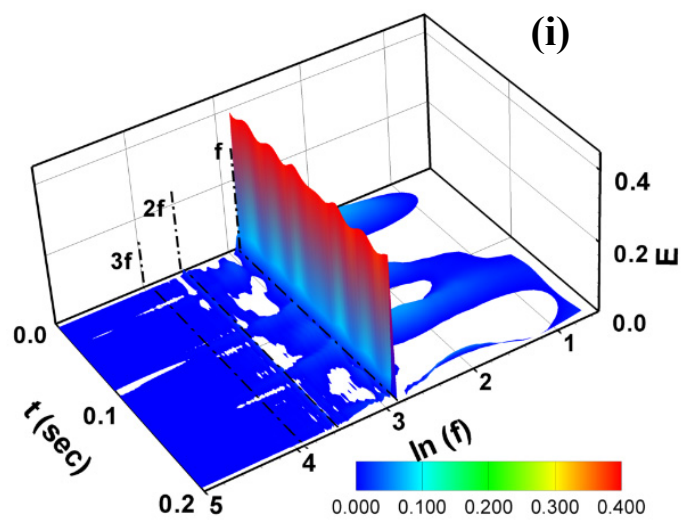

(ii)



(iii)

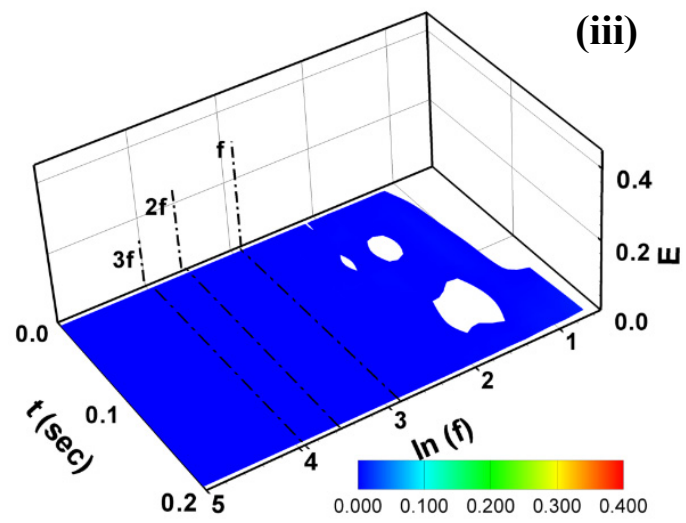

Figure 4.31: Choi-William transformation at $18 \mathrm{~Hz}$ actuation frequency in downstream direction along the synthetic jet centerline at three different stream wise locations $X / D$ or $X / W=3,8$ and 12 for circular orifice (D10) are shown in $((a),(b) \&(c))$ and for square orifice(AR1) are shown in ((i), (ii) \& (iii)). 
AR2

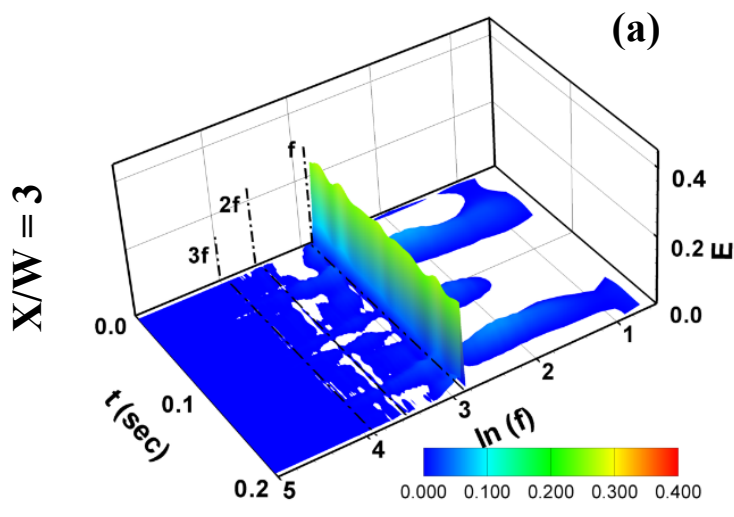

(b)

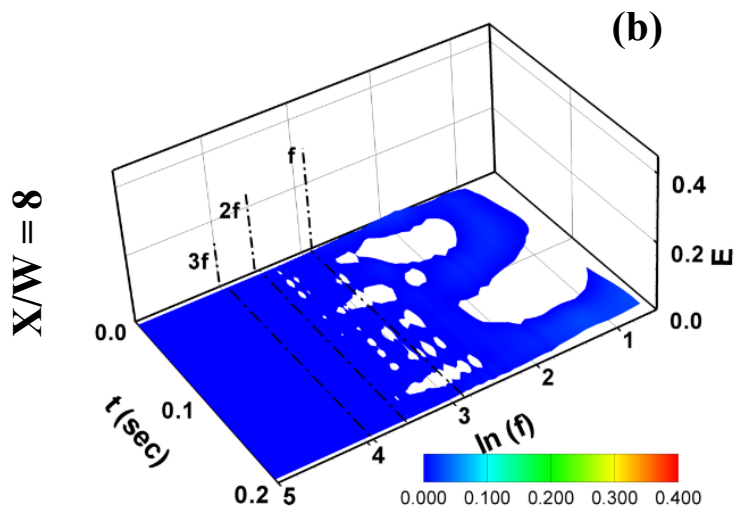

(c)

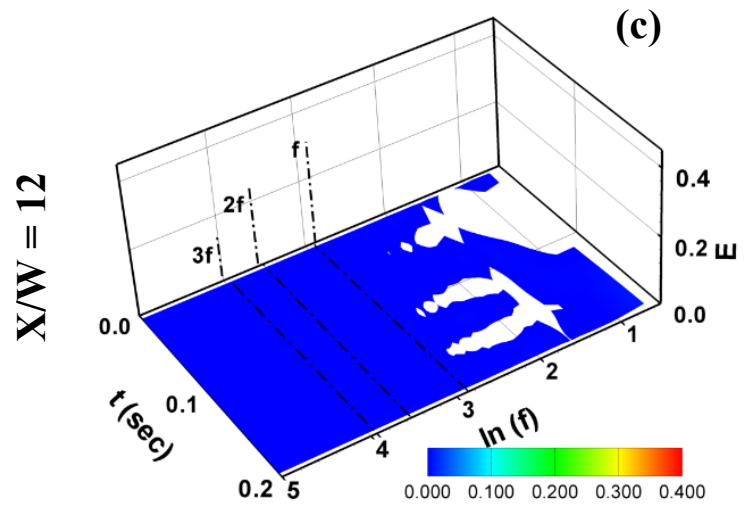

AR4

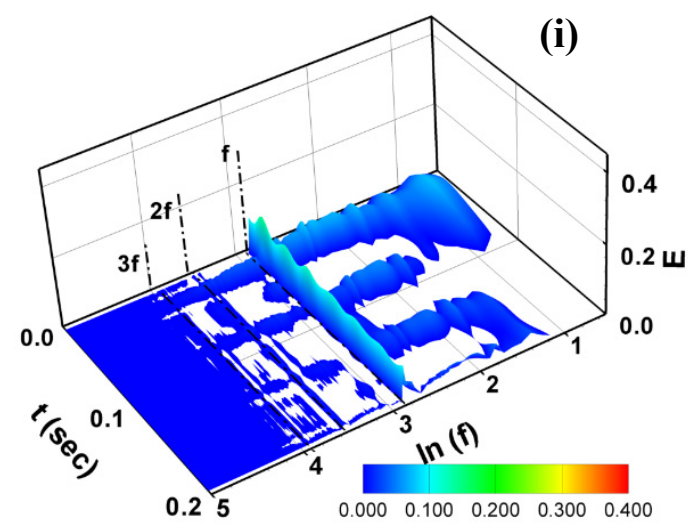

(ii)

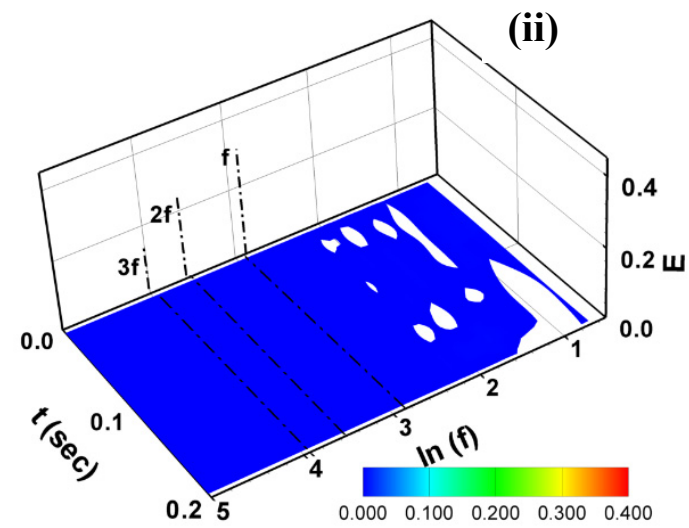

(iii)

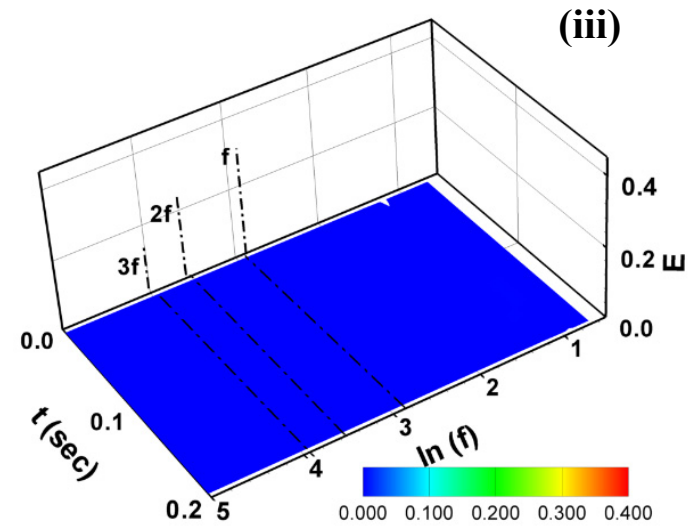

Figure 4.32: Choi-William transformation at $18 \mathrm{~Hz}$ actuation frequency in downstream direction along the synthetic jet centerline at three different stream wise locations $X / W=3,8$ and 12 for rectangular orifice (AR2) are shown in ((a), (b) \& (c))and for rectangular orifice(AR4) are shown in ((i), (ii) \& (iii)).

In between the two harmonic frequencies, the shape of rib for circular orifice D10 is different than that of non-circular orifices AR1, AR2 and AR4 in the sense that rib 
decreases monotonically for D10 orifice whereas it has curved shape for AR1, AR2 and AR4. This may be linked to the periodic fluctuation due to corner effect or axial switching in velocity at the synthetic jet centerline. In comparison to AR1 and AR2 orifice, there is no formation of vortex rings and thus the wavelet distribution is filled with sparse distribution of small-scale vortices of different length scale for AR4 orifice. It also indicate that there is no periodicity in time domain. The wavelet transformation also shows some randomly distributed streaks of varying width, which indicates that the flow field is chaotic and close to turbulent.

\subsection{Closure}

In this chapter, the characteristics of circular, square, and rectangular orifices are discussed with the help of LIF images and velocity field measured using S-type hot film. Different patterns of flow structure are observed for circular orifices of 8,10 and $13 \mathrm{~mm}$ diameter are: vortex ring with partial suction of ejected fluid, formation of distinct vortex ring, formation of coherent trailing jet and turbulent synthetic jet. The time-averaged velocity magnitude increases from orifice exit to a maximum value within the suction region near the orifice, remains at a higher value until the vortex convects and expands which subsequently decreases monotonically for distinct vortex ring and steeply for turbulent synthetic jet as vortex ring slow down and breaks.

The behaviour of rectangular orifices are different from that of the circular orifice due to presence of corners, which significantly affects the vortex formation and its propagation in downstream direction. For rectangular orifices, at low Reynolds number, the propagation velocity of newly formed vortex rings is lower than that of vortex ring at higher Reynolds number. Therefore, shape of the vortex ring is extensively governed by the suction period of following cycle causing a wide variation in the flow structure. After 
traveling a certain distance downstream, the flow pattern changes from laminar to chaotic or turbulent more rapidly than the circular orifices at same actuation frequency and at equivalent hydraulic diameter. The different patterns of flow structure observed for rectangular orifices are vortex ring with axial switching, vortex ring bifurcation with and without the left over fluid at splitting point, the partial suction of ejected vortex ring and the exploding of vortex ring into multiple tiny rings very near to the orifice exit. Based on vortex size measurement, the maximum number of axial switching is in between 2 to 3 before the vortex rings breakdown or disappear. The synthetic jet spreads more in the plane perpendicular to the longer side of orifice.

In velocity time trace, the pulsatile nature are seen in the form of peaks or humps evenly distributed in time at the rate of actuation frequency. In Fast Fourier transformation, the presence of peaks at harmonics of actuation frequency indicates the existence of coherent structures. Maximum numbers of peaks at harmonic frequencies are observed for $6 \mathrm{~Hz}$ actuation frequency in comparison to 1 and $18 \mathrm{~Hz}$ actuation frequency. However, the sustainability of peaks for a longer distance in downstream is observed for D10 orifice in comparison to AR1, AR2 and AR4 orifices. In wavelet transformation (joint timefrequency representation), the total energy of the velocity signal is primarily distributed in the form of ribs and bands. Ribs parallel to the frequency axis show the energy distribution of the vortex ring in frequency domain and bands parallel to the time axis shows periodicity or pulsating nature of coherent structures at different harmonics of actuation frequency. The ribs are separated and decrease monotonically if the vortex rings are laminar and well separated. Synthetic jets with trailing jet have a band parallel to time axis at different harmonics. For turbulent and chaotic flow, first harmonic frequency have maximum share of energy in comparison to the energy distributed at higher order harmonics. 


\section{Chapter 5}

\section{Bifurcation in synthetic jet with rectangular shape orifice}

In the previous chapter, the characteristics of synthetic jets with square and rectangular shape orifice have been discussed. An interesting phenomenon of vortex ring bifurcation is observed for rectangular shape orifice of aspect ratio two without any other mode of excitation except the periodic axial actuation. Since the bifurcation of synthetic jet have better mixing and spreading compared to non-bifurcation case, it exhibits a possible application in flow control devices. The aspect ratio is an important parameter in determining the jet-spreading rate of non-circular vortex rings. Increasing the aspect ratio increases the variation in self-induced velocity of different segments of vortex ring. The difference in induced velocity enhances the mixing of vortex ring with surrounding fluids. Trentacoste and Sforza ( 1967) reported that enhancement in entrainment for free jet having aspect ratio larger than five is not significant. Schadowk et al. (1984) while experimenting on gas-generator nozzles of various aspect ratios suggested that the fine scale mixing is optimized for nozzles having aspect ratio in between $2: 1$ to $3: 1$. The current chapter focuses on detailed study of rectangular synthetic jet of aspect ratio two in still water.

The non-circular vortex ring of aspect ratio more than one has two different types of vortex deformation: one that leads to axial switching and the other, which leads to 
bifurcation. The history of bifurcation shows two different kinds of bifurcation method: (a) periodically deviating the path of vortex ring from the jet centerline by imposing combined axial and flapping/helical mode of excitation and (b) splitting of vortex ring into two smaller vortex rings. While the first mode is largely seen for circular vortex rings, the second one finds its application for non-circular vortex rings. In dual mode of excitation, the combined axial and flapping forcing creates a periodic array of vortex rings, which are alternatively shifted in the radial direction. The helical disturbances reach their maximum amplification at the end of the potential core. As a result, the vortex rings tilts and propagate along two different trajectories (Danaila and Boersma, 2000). Parekh et al. (1988) and Reynolds et al. (2003) reported that a round jet could be bifurcated by simultaneously imposing helical and axial modes of forcing. Gohil et al. (2010) investigated the mechanism of bifurcation in jet using Direct Numerical Simulation. By enforcing an axial excitation at the preferred mode of frequency (axial) and a helical excitation at the sub-harmonic mode frequency for both the circular and square orifice, they reported that in dual mode excitation, both circular as well as square jets splits into two separate jet on the bifurcating plane. The angle between the two separated jets is greater for circular jets than that of the square jet. Silva and Metais (2002) studied flapping excitation at preferred and subharmonic modes for various Reynolds numbers and concluded that the maximum spreading rate is achieved by applying a sub-harmonic frequency.

In the second case, the bifurcation is obtained by combined effect of orifice shape and velocity for free jet/single vortex ring. However, in case of synthetic jet the actuation frequency is also added as third controlling parameter. Here, the prime source of vortex splitting is variation in the self-induced velocity of vortex ring due to variation in curvature of nozzle or orifice (IIO et al. (2006) and Jiao Jian et al. (2005)), which leads to the phenomenon of axial switching (Krothapalli et al. (1981), Ho and Gutmark (1987) and 
Zaman (1996)). However, the mechanism of vortex splitting will be different for bifurcation in free jet, bifurcation of single vortex ring, bifurcation of single vortex ring followed by a suction stroke or the bifurcation in a train of vortex ring (synthetic jet). In free jets, initially the axial switching followed by vortex ring splitting is reported by Arms and Hama (1965), Dhanak and de Bemardinis (1981), Hussain and Husain (1989), Grinstein (2001) and Zaman (1996) for elliptical or rectangular shaped orifice or nozzle. The splitting of vortex ring in free jet differs from single vortex ring in the sense that in free jet there is continuous supply of vorticity in form of continuous jet issuing from the nozzle whereas in single vortex ring case it is stopped after certain duration. Stopping of feeding fluid after certain duration creates a single vortex ring with or without trailing jet that depends on the magnitude and supply rate of vorticity. In this case, no perturbation exists in environment before the formation of vortex ring and hence there is no influence of environment on the behavior of vortex ring. Heertch (1976) studied the jet flow for rectangular nozzle of aspect ratio in the range of 1 to 20, while keeping the cross-sectional area constant. The vortex ring is formed by electromagnetically driven actuator where the forward stroke varies between 0.18 to 1.0 second, giving Reynolds number in between 144 to 1080 based on hydraulic diameter of nozzle. On using the forward stroke only (single vortex ring), he reported a clear splitting of vortex ring for $9 \geq A R \geq 7$ in major axis plane of nozzle. For AR $>9$, he observed that as the aspect ratio grows one needs an everincreasing stroke length in order to generate splitting vortices. Oshima et al. (1988) for single vortex ring observed partial bifurcation, because the vortex ring collapse just after the splitting and no isolated path for splitted vortex rings is formed. However, for his case the bifurcation plane is minor axis plane of orifice instead of major axis plane. It means that the bifurcation can happen in both minor and major axis plane of orifice depending on the orifice shape and controlling parameter. Kiya et al. (1992) with help of numerical 
simulation and experimental results showed the bifurcation of single pseudo-elliptical vortex ring in both planes (minor and major axis plane) for aspect ratio in between 2 to 20 . At constant Reynolds number equal to 1500 (based on circulation), four different patterns are reported and they are: pseudo-periodic axial switching for $2<\mathrm{AR}<4$, axial switching followed by vortex splitting in minor axis plane for $5<\mathrm{AR}<8$, vortex ring splitted without axial switching in major axis plane for $8.9<\mathrm{AR}<11$ and vortex ring splitted into three distorted vortex ring without experiencing axial switching for $11<\mathrm{AR}<20$. Auerbach and Grimm (1994) compared his results with that of Heertch (1976) for AR = 3 with slightly different experimental conditions in terms of stroke length, orifice cross-sectional area and velocity profile of actuation. They reported similar observation of vortex splitting in major axis plane based on divergence or convergence of counter rotating vortices in captured images in major axis plane of nozzle alone. In his case, the lower value of Reynolds number promotes the convergence whereas increasing the value of Reynolds number leads to splitting and divergence. However, no quantitative information or further detail in another orthogonal plane is reported. As per author's knowledge, there is no literature that reports the process of bifurcation in synthetic jet. Since in synthetic jet the forward stroke is followed by suction stroke and vice - versa in a cyclic manner, the built up flow field of vortex ring continuously changes and influences the behavior of newly born vortex ring. Therefore, the factors controlling the bifurcation process behaves differently in case of synthetic jet.

In the present work, a rectangular orifice of aspect ratio two $\left(7 \times 14 \mathrm{~mm}^{2}\right)$ with orifice thickness of $3 \mathrm{~mm}$ and sinusoidal actuation of diaphragm by electromagnetic actuator have been investigated. The details of experimental parameters are given in Table 5.1. The calculation details of hydraulic diameter, Reynolds number, Strouhal number and other parameters are given in Chapter 3. The hydraulic diameter of the orifice is equal to 
$9.33 \mathrm{~mm}$. The LIF images are acquired in major and minor axis planes for qualitative study.

Two components of velocity measurement are reported using Laser Doppler Velocimetry.

The synthetic jet unit is mounted on the sidewall of the water tank (quiescent flow set-up discussed in Chapter 3). The orifice orientation and axis notation used are shown in Figure 5.1.

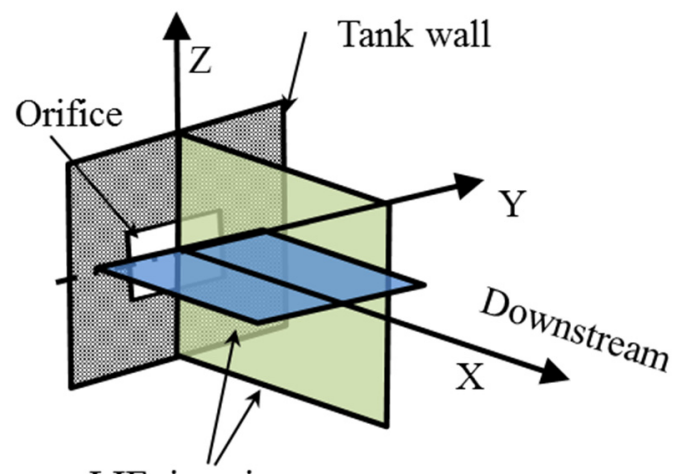

LIF imaging planes (a)
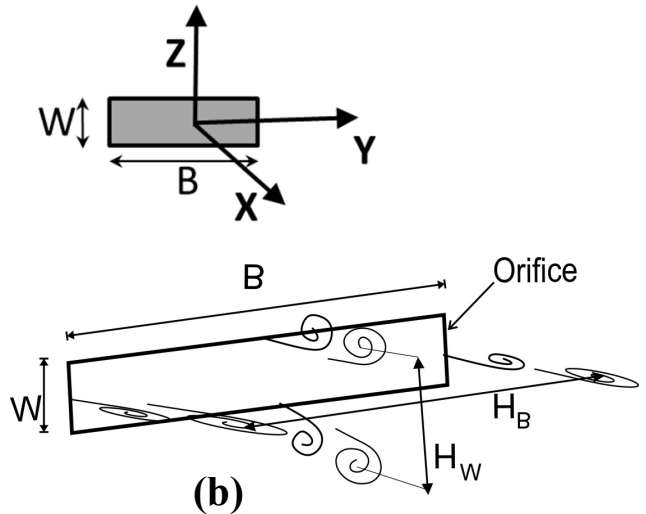

(b)

Figure 5.1: Schematic of (a) orifice orientation and (b) axis notations.

Table 5.1: Details of experimental variables.

\begin{tabular}{|c|c|c|c|c|c|c|}
\hline \multirow[t]{2}{*}{ Cases } & \multicolumn{2}{|c|}{$\begin{array}{l}\text { Actuation frequency } \\
\text { (f) } \mathrm{Hz}\end{array}$} & \multirow{2}{*}{$\begin{array}{c}\text { Reynolds } \\
\text { number } \\
\text { (Re) }\end{array}$} & \multirow{2}{*}{$\begin{array}{c}\text { Strouhal } \\
\text { number } \\
\left(\mathbf{S}_{t}\right)\end{array}$} & \multirow[t]{2}{*}{$\mathbf{L} / \mathbf{D}_{\mathbf{h}}$} & \multirow[t]{2}{*}{ Remark } \\
\hline & Actual & Round-off & & & & \\
\hline I & 1.02 & 1 & 275 & 0.33 & 1.51 & \multirow{3}{*}{$\begin{array}{l}\text { Bifurcation } \\
\text { or vortex splitting }\end{array}$} \\
\hline II & 1.60 & 2 & 1101 & 0.13 & 3.83 & \\
\hline III & 2.56 & 3 & 1309 & 0.17 & 2.94 & \\
\hline IV & 1.02 & 1 & 625 & 0.15 & 3.42 & \multirow{3}{*}{$\begin{array}{l}\text { No bifurcation } \\
\text { or axial switching }\end{array}$} \\
\hline V & 1.60 & 2 & 1348 & 0.10 & 4.69 & \\
\hline VI & 2.56 & 3 & 2038 & 0.11 & 4.57 & \\
\hline
\end{tabular}




\subsection{Flow visualization}

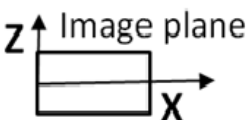

Side view (XZ-plane)
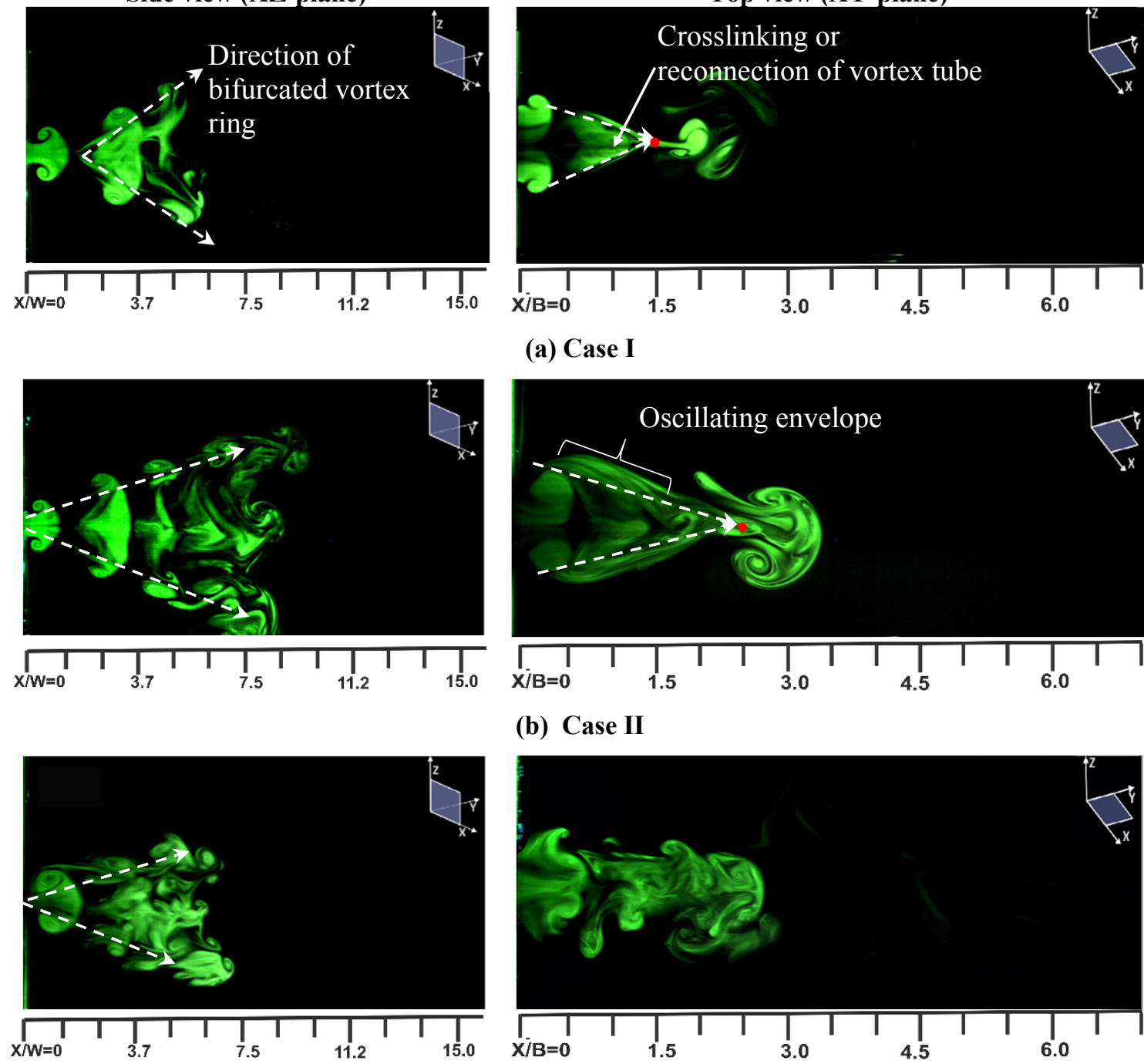

(c) Case III

Figure 5.2: Flow visualization images of synthetic jet in XZ-plane (side view) and in XY-plane (top view) for bifurcation cases (a) Case 1: $f=1 \mathrm{~Hz}, \mathrm{Re}=275, \mathrm{~L} / \mathrm{D}_{\mathrm{h}}=1.51$, (b) Case II: $\mathrm{f}=\mathbf{2} \mathrm{Hz}$, $\mathrm{Re}=$ $1101, \mathrm{~L} / \mathrm{D}_{\mathrm{h}}=3.83$, and (c) Case III: $\mathrm{f}=3 \mathrm{~Hz}, \mathrm{Re}=1309, \mathrm{~L} / \mathrm{D}_{\mathrm{h}}=2.94$. (*It may be noted that the images are not exactly at same instant of time).

Figure 5.2 shows the LIF images of vortex ring at actuation frequency of 1,2, and

$3 \mathrm{~Hz}$, where vortex ring splits and Figure 5.3 shows the cases where vortex ring proceeds towards axial switching. The LIF images of vortex ring captured from side (XZ-plane or 
minor axis plane) and from the top of the tank (XY-plane or major axis plane) are shown. The images are not acquired at same instant of time for side and top view. However, the numbers of cycles from the starting of synthetic jet are approximately same for different actuation frequency cases.

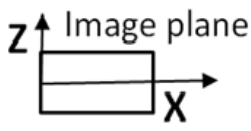

Side view (XZ-plane)
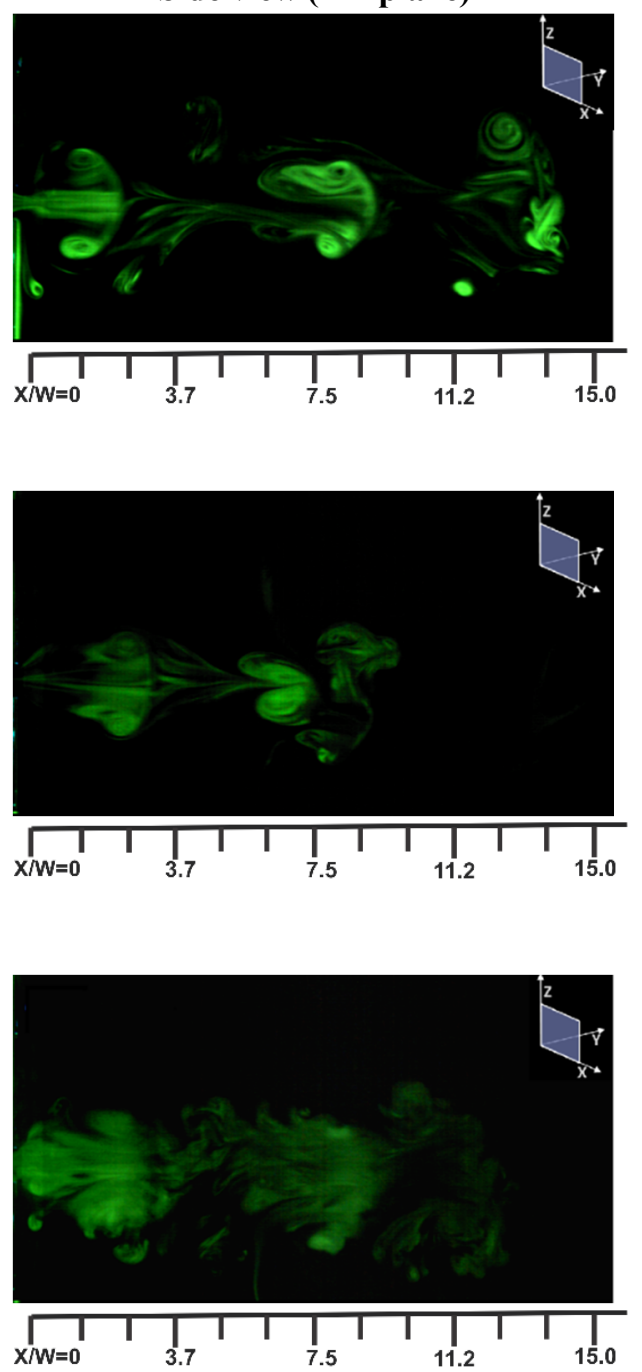
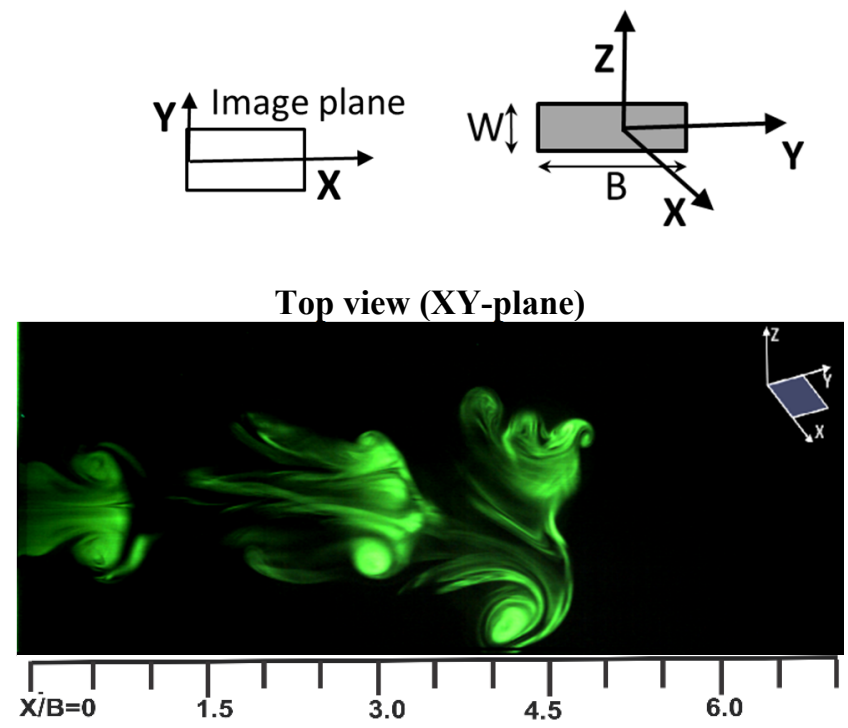

(a) Case IV

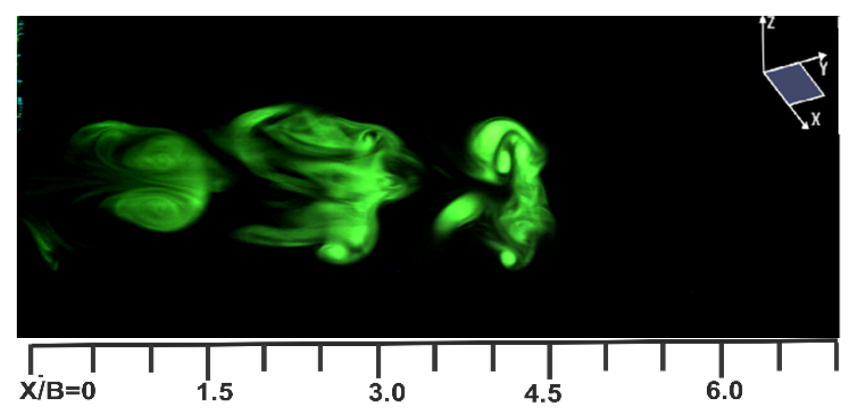

(b) Case V

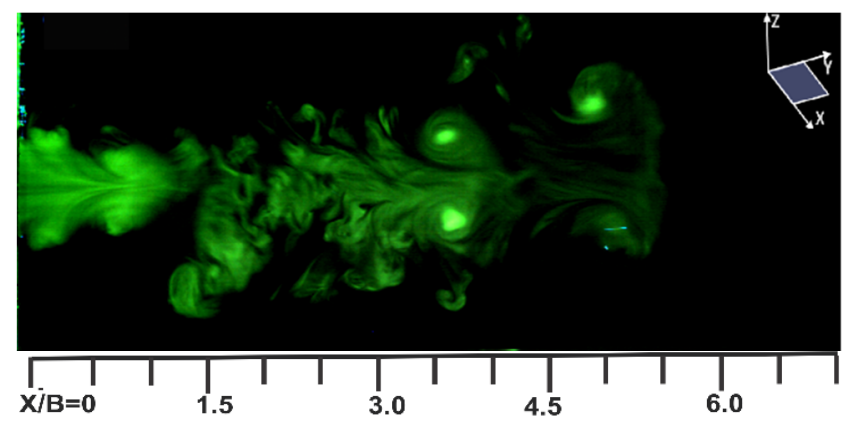

(c) Case VI

Figure 5.3: Flow visualization images of synthetic jet in $X Z$-plane (side view) and in $X Y$-plane (top view) for non- bifurcation cases at (a) Case IV: $f=1 \mathrm{~Hz}, \mathrm{Re}=625, \mathrm{~L} / \mathrm{D}_{\mathrm{h}}=3.42$, (b) Case $\mathrm{V}: \mathrm{f}=2 \mathrm{~Hz}$, $\operatorname{Re}=1348, \mathrm{~L} / \mathrm{D}_{\mathrm{h}}=4.69$ and (c) Case VI: $\mathrm{f}=3 \mathrm{~Hz}, \mathrm{Re}=2038, \mathrm{~L} / \mathrm{D}_{\mathrm{h}}=4.57$. (* It may be noted that the images are not exactly at same instant of time). 
In Figure 5.2 (a), the splitting of vortex ring is shown for Case I where the vortex ring bifurcates in XZ-plane and moves at an angle of $62^{\circ}$ with each other in downstream direction. Figure 5.2 (b) shows the vortex splitting with splitting angle equal to $46^{0}$ for Case II with some portion of vortex ring left at the center during the splitting, forming a $\Psi$ - shape of flow structure. In top view, the diverging angles of counter rotating vortices from orifice exit to the point of merging are approximately $56^{0}$ and $40^{\circ}$ for Case I and Case II respectively. In Case III (Figure 5.2(c)), the splited vortex rings have a smaller splitting angle equal to $34^{\circ}$ and no clear or distinct splitting is observed in the side view. Here, the process of bifurcation is initiated but before the completion of bifurcation or splitting, the vortex ring breaks into smaller vortices and moves all around randomly. Similar observation was also reported by Oshima et al. (1988) for elliptical vortex ring of aspect ratio three in air using Schlieren method. They reported that at the point of vortex reconnection (see Figure 5.2 (a)), counter rotating vortices come closer and cancel the outer part of adjacent vortex filament. At the same time, a breakdown is initiated at the crosslinking region generating abrupt turbulence in the vortex filament. These turbulence structures propagate around the remaining vortex tube from the interaction region and break the vortex ring into tiny vortices, which prevents the bifurcation of vortex ring. In all three bifurcation cases I, II and III, the vortex rings splits or try to split just after the first axial switching in minor axis plane of orifice. The trend for vortex ring splitting obtained above for rectangular synthetic jet of aspect ratio two is in good agreement with Kiya et al. (1992) for single pseudo-elliptical vortex ring of aspect ratio two. Since, after the first axial switching the major axis of vortex ring becomes minor and vice-versa, the shorter side of vortex rings that is initially in major axis plane (XY-plane) switches in minor axis plane (XZ-plane) of orifice. Therefore, the vortex reconnection and splitting occurs in XZ-plane perpendicular to the longer side of orifice. Another important observation is the location of 
vortex crosslinking shown by dots in the top view of LIF images (see Figure 5.2). This location is about $\mathrm{X} / \mathrm{B}=1.5$ for Case I and $\mathrm{X} / \mathrm{B}=2.5$ for Case II. It indicates that the point of vortex splitting is not fixed, and varies depending on the flow control parameters. In side view, there is no sign of vortex leap forging (following vortex ring penetrates inside the leading vortex ring and moves ahead due to velocity difference). However, the top view shows that the newly formed counter rotating vortices penetrate into the previous vortex rings (see Figure 5.2 (b)). In this process, they get squeezed in the beginning and finally consume inside the envelope of previous rings. Careful examination of video corresponding to the LIF images in XY-plane shows that the piercing action of converging vortices pushes the envelope of fluid away from the synthetic jet centerline in the region between $\mathrm{Y} / \mathrm{B}=$ 0.5 to 1.5 during convection. As the counter rotating vortices moves ahead, the envelope moves down to fill the gap. This cycle is repeated and the envelope oscillates in Y-direction for each passes of counter rotating vortices (see Figure 5.2 (a) and (b) top view). In Figure 5.3, at the same actuating frequency 1,2 and $3 \mathrm{~Hz}$, the diaphragm displacement is increased so that synthetic jet experiences axial switching instead of vortex ring bifurcation. The vortex rings are isolated and the trailing jet is negligible for Case IV but a small amount of trailing jet forms for Case $\mathrm{V}$, which interacts with the following vortex rings. The increased frequency from 1 to $2 \mathrm{~Hz}$ decreases the spacing and increases the interaction which is seen by early breakdown of vortex ring. In Case VI, the images in both the planes show a significant amount of trailing jet.

\subsection{Vortex size}

The vortex size variation is an important criterion to decide the vortex growth and behavior of vortex ring. The trajectory of counter rotating vortices are measured from the sequence of LIF images in XZ and XY-plane and plotted in Figure 5.4. The formation of trailing jet is also seen in some cases but the global flow pattern largely depends on the vortex 
dynamics of leading vortex ring. Hence, the separation distance between the cores of counter rotating vortices of leading vortex ring provides useful information about the formation and evolution of synthetic jet. The measured values of separation distances are plotted in both, time and space domain for all six cases as shown in Figure 5.4.


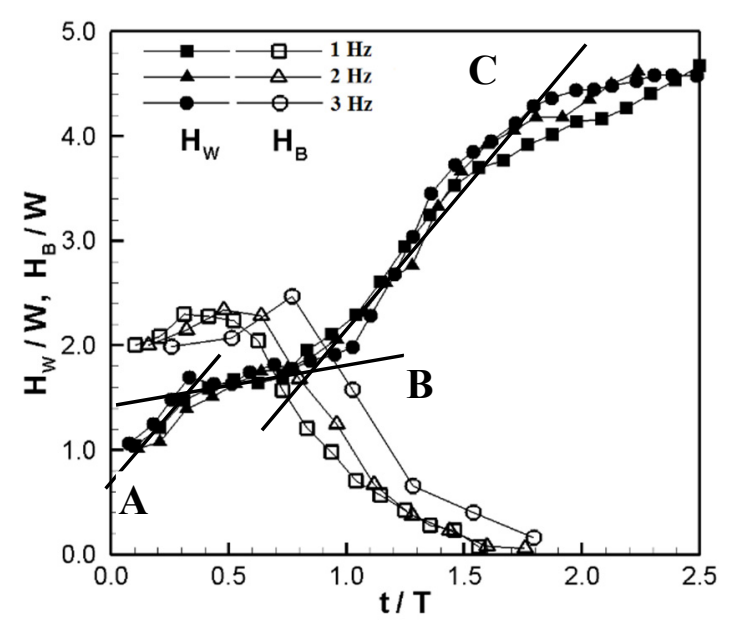

(a) Bifurcation cases



(c) Non-bifurcation cases

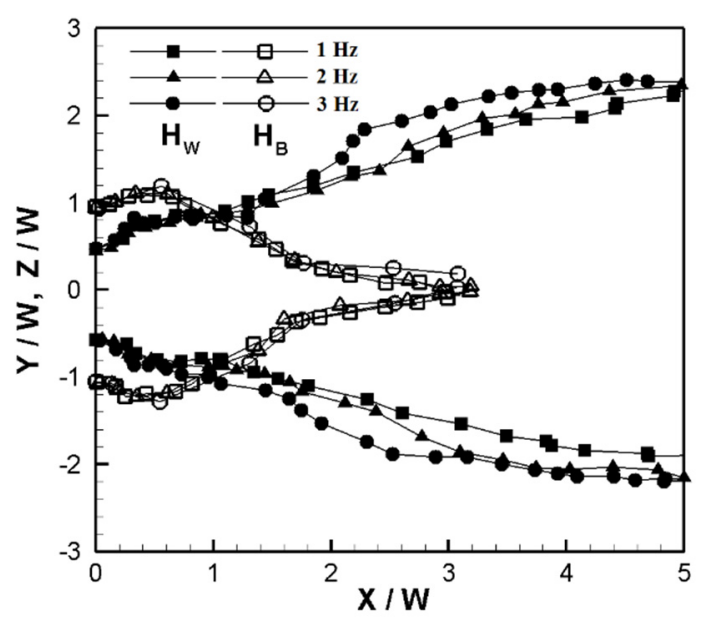

(b) Bifurcation cases

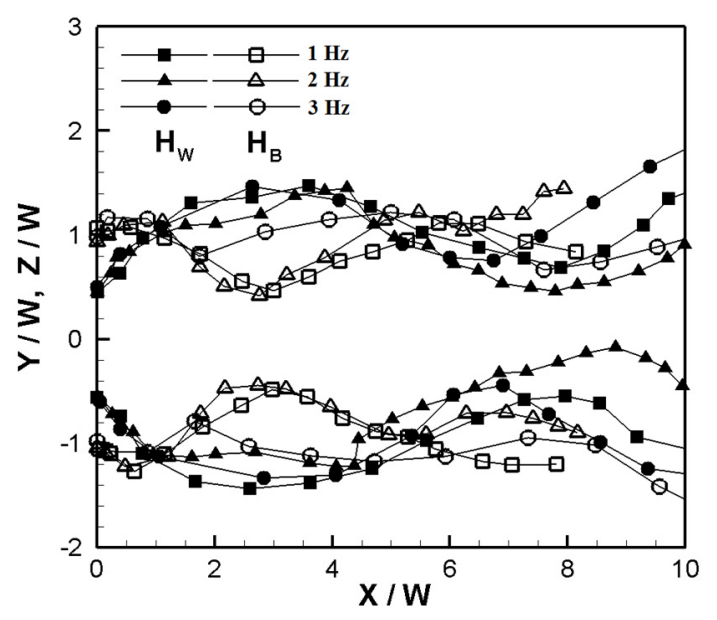

(d) Non-bifurcation cases

Figure 5.4: Separation distance ((a) and (c)) between the cores of counter rotating vortices in time domain and position of cores $((b)$ and(d)) in space domain measured from the sequency of LIF images in XZ and XY-plane for bifurcation cases (I, II and III) and for non-bifurcation cases (IV, V and VI). ( ${ }^{*}$ The values of $\mathrm{H}_{\mathrm{B}}$ and $\mathrm{H}_{w}$ are shifted in $\mathrm{Y}$ axis by step size equal to 2 in figure (c) for calarity). 
In the bifurcation cases, I, II and III in Figure 5.4 (a) at 1, 2 and $3 \mathrm{~Hz}$ actuation frequency, the separation distance between counter rotating vortices in both the orthogonal planes follow the same trajectory in time domain. The first axial switch-over after which the shorter side becomes larger and vice-versa occurs between $\mathrm{t} / \mathrm{T}=0.6$ to 0.9 in time domain and nearly at $\mathrm{X} / \mathrm{W}=1$ in downstream direction. The trajectory of counter rotating vortices in XZ-plane for bifurcating cases have three distinct regions represented by three straight lines as shown in Figure 5.4 (a). The regions are: (i) the time in which shear layer rolls into vortex ring (line-A), (ii) the time after first axial switching when the longer side of vortex ring interconnects and then splits (line-B) and (iii) the splitted vortex rings moving at an angle (line-C). It has been observed that the size of vortex ring in XZ-plane remains almost stagnant for some time (line-B) but it increases and decreases in XY-plane with a small magnitude. It means that during the vortex reconnection and splitting period, the size of vortex ring in bifurcation plane is on hold. Once the splitting is completed, the splitted vortex rings moves at an angle to each other seen as higher separation distance between the upper and lower vortex rings.

In general, the rectangular shape vortex ring expands in the direction of minor axis and contract in major axis due to differences in their self-induced velocity. After some time the major axis of vortex ring becomes minor axis, and minor axis switches to major axis. At this stage, the first axial switchover is completed and it advances towards the second switchover. Normally, the first axis switch-over is expected in the case where vortex splits in the direction of major axis for larger aspect ratio orifices. The vortex size expansion, entrainment with surrounding fluid, growth of core instability and decreasing velocity restricts the number of total axial switching before the vortex breakdown. A maximum of third axial switching is reported by Hussain and Husain (1989) for elliptical jets. They also reported that the third axial switching is usually faint and may not be easily detectable. Ho 
and Gutmark (1987) also reported maximum of three axial switching in elliptical nozzle of aspect ratio two on free air jet. A maximum of three axial switching is also observed in Figure 5.4 for Case IV, V and VI. The trajectory of counter rotating vortices shown in Figure $5.4(\mathrm{~d})$, shows the size of major and minor axis of vortex ring are equal at $\mathrm{X} / \mathrm{W} \sim$ 1. However, the location of first axial switch-over vary significantly for Case VI in comparison to Case IV and V which is likely to be linked with the strong trailing jet observed in Figure 5.4 (c).

\subsection{Vortex evolution}

The time stamp images of vortex evolution and bifurcation process are shown in minor axis plane (XZ) and in major axis planes (XY) in Figure 5.5 and Figure 5.6 respectively. The maximum width and orientation of counter rotating vortices are also indicated in the figure using line segment. At $\mathrm{t} / \mathrm{T}=0.4$ in Figure 5.5, the fluid ejected from the orifice center is ahead than the fluid curling to form the vortex tube near the orifice wall. However, at the same instant of time in Figure 5.6, the fluid ejected at the orifice center lags behind the fluid at orifice wall. The other observation is curling of shear layer to form the vortex tube in $\mathrm{XY}$-plane is weaker than that of curling of shear layer in XZ-plane at same time. The differences in curling radius of vortex tube formation in $\mathrm{XY}$ and $\mathrm{XZ}$-plane forms vortex ring of varying tube radius and velocity. In result, the axis of vortex tube stretches and tilted (indicated by small straight lines drawn just above the upper counter rotating vortices) in XZ-plane and contracts in XY-plane. At the end of forward stroke, when the vortex ring detached itself from the orifice and moves downstream, nearly at the same moment the backward stroke of the diaphragm initiate the suction stroke. Hence, the fluid moving towards the orifice cavity squeezes the newly formed vortices at its rear end and changes the shape and orientation of vortex tube as shown in Figure 5.5 at $\mathrm{t} / \mathrm{T} \sim 0.6$. Due to changes in orientation and shape, the counter rotating vortices moves away from the synthetic jet 
centerline in XZ-plane in Figure 5.5 and comes closer in XY-plane at $\mathrm{t} / \mathrm{T} \sim 0.8$ and 1.0 in Figure 5.6. In XY-plane, the separation distance between the counter rotating vortices decreases continuously with time and the left over portion of former vortex rings creates an envelope over the newly formed vortices and squeeze them towards the synthetic jet centerline. The contraction of counter rotating vortices at $\mathrm{t} / \mathrm{T}=1.2$ to 1.6 in Figure 5.6 leads to vortex tube reconnection in XY-plane and bifurcation in XZ-plane as seen in Figure 5.5. The sequence of images reveals that the thickness of envelope increases initially and then remains unchanged in time.
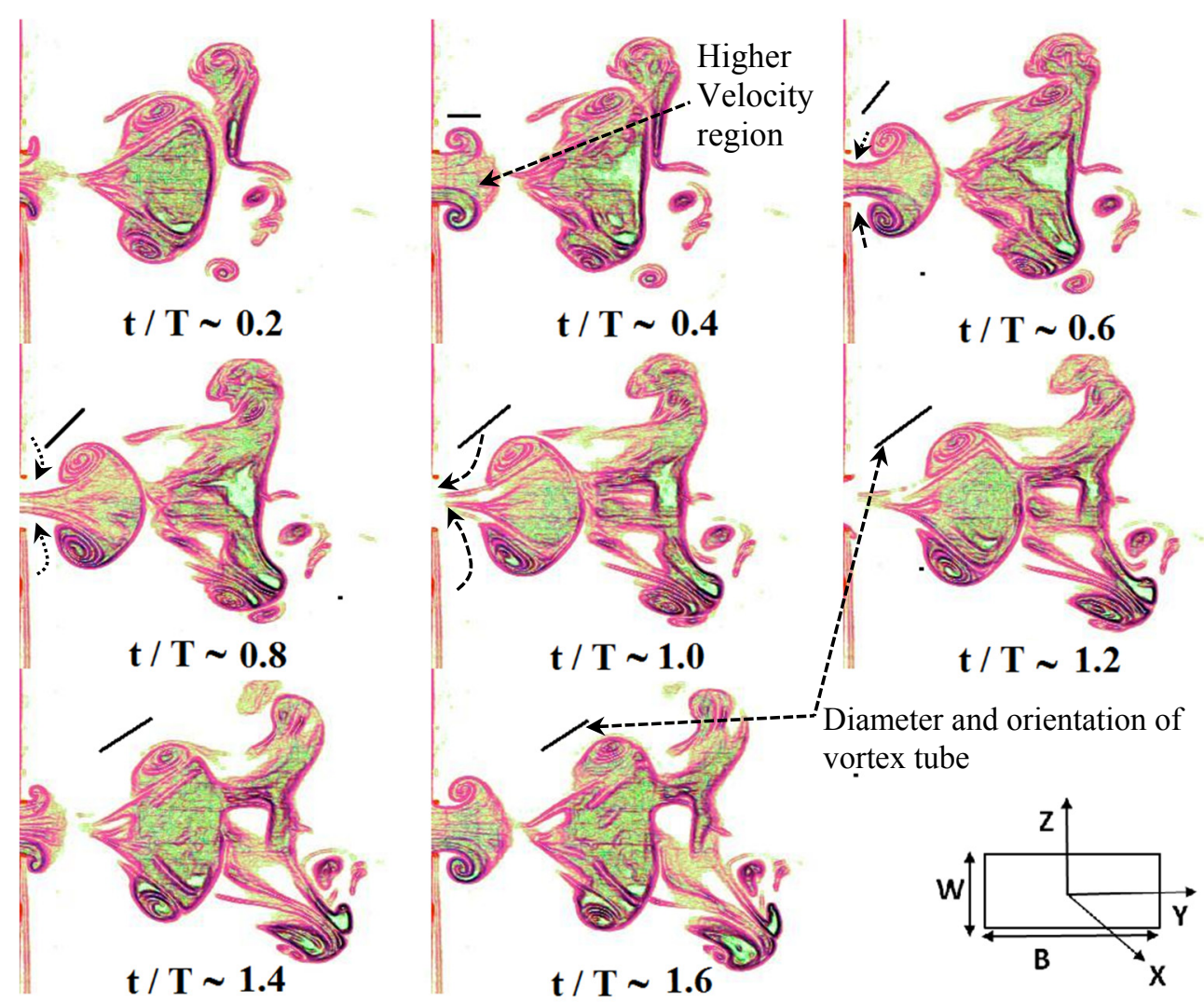

Figure 5.5: Sequence of LIF images showing vortex evolution and bifurcation process at different time stamps in XZ-plane for Case I. 


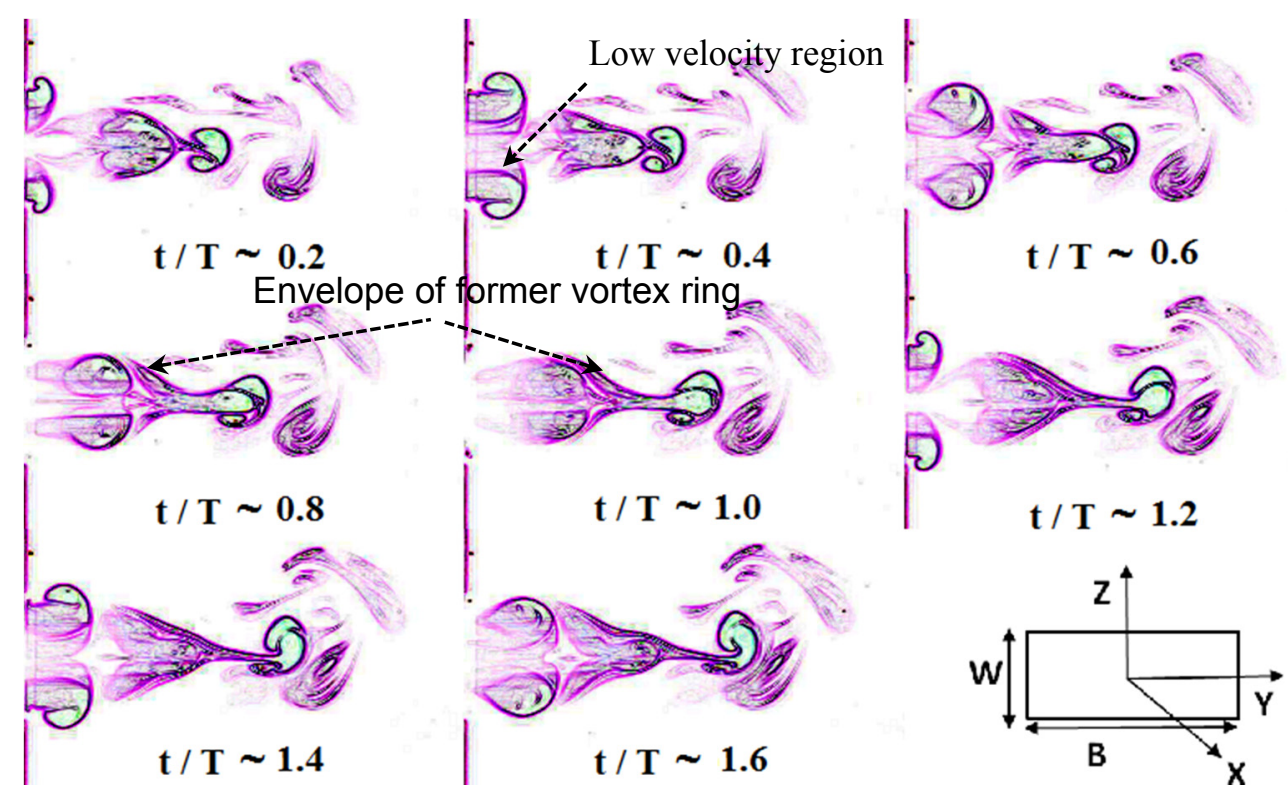

Figure 5.6: Sequence of LIF images showing vortex evolution and bifurcation process at different time stamps in XY-plane for Case I.

Based on the experimental observations and literature, the schematic of axial switching and bifurcation of rectangular shape vortex ring are shown in Figure 5.7. At the orifice exit, the shorter sides of vortex tube are labeled as $\mathrm{S}_{1}$ and $\mathrm{S}_{2}$ and longer sides are labeled as $1_{1}$ and $1_{2}$. The vortex ring experiences axial switching or splitting depending on the flow condition imposed. As soon as the vortex detaches itself from the orifice, the shorter side $\left(S_{1}, S_{2}\right)$ moves faster than the longer side $\left(l_{1}, l_{2}\right)$. The differences in velocity produce strain within the vortex ring. To eliminate the regions that have higher strain rate, the shorter side of vortex ring moves towards the vortex center, where as the longer side of vortex tube moves away from the center. If the vortex ring have enough energy and comparatively lower velocity difference between the shorter and longer side, the shorter side becomes longer side and vice versa. This process is continue till the vortex breakdown. This process is shown in Figure 5.7 (a), where the sorter side moves ahead and towards the vortex center pushing the longer side away from the orifice (shown by arrowheads) and in due time the shorter side $S_{1}, S_{2}$ becomes longer, and longer side $1_{1}, l_{2}$ becomes shorter. In case of bifurcation, the shorter side of vortex ring comes very close to each other due to 
higher velocity difference as compared to the axial switching of vortex ring. The envelope formed from previously ejected vortex rings also supports the interconnection of vortex tube, which is called as crosslinking or connect-and-cut process. The process of vortex splitting is the result of crosslinking of vortex tube. Melande and Hussain (1989) suggested that the crosslinking of vorticity is a cut and connect process in which the core deformation and stretching is followed by annihilation and bridging, which is completed by threading and splitting. Yao and Xungang (1997) observed that opposite-signed vorticity (here counter rotating vortices of vortex ring) inside the contact zone gets cancelled out by viscous cross-diffusion. These cancelled vortex lines of vortex tube gets connected with their counterpart and forms isolated vortex rings.

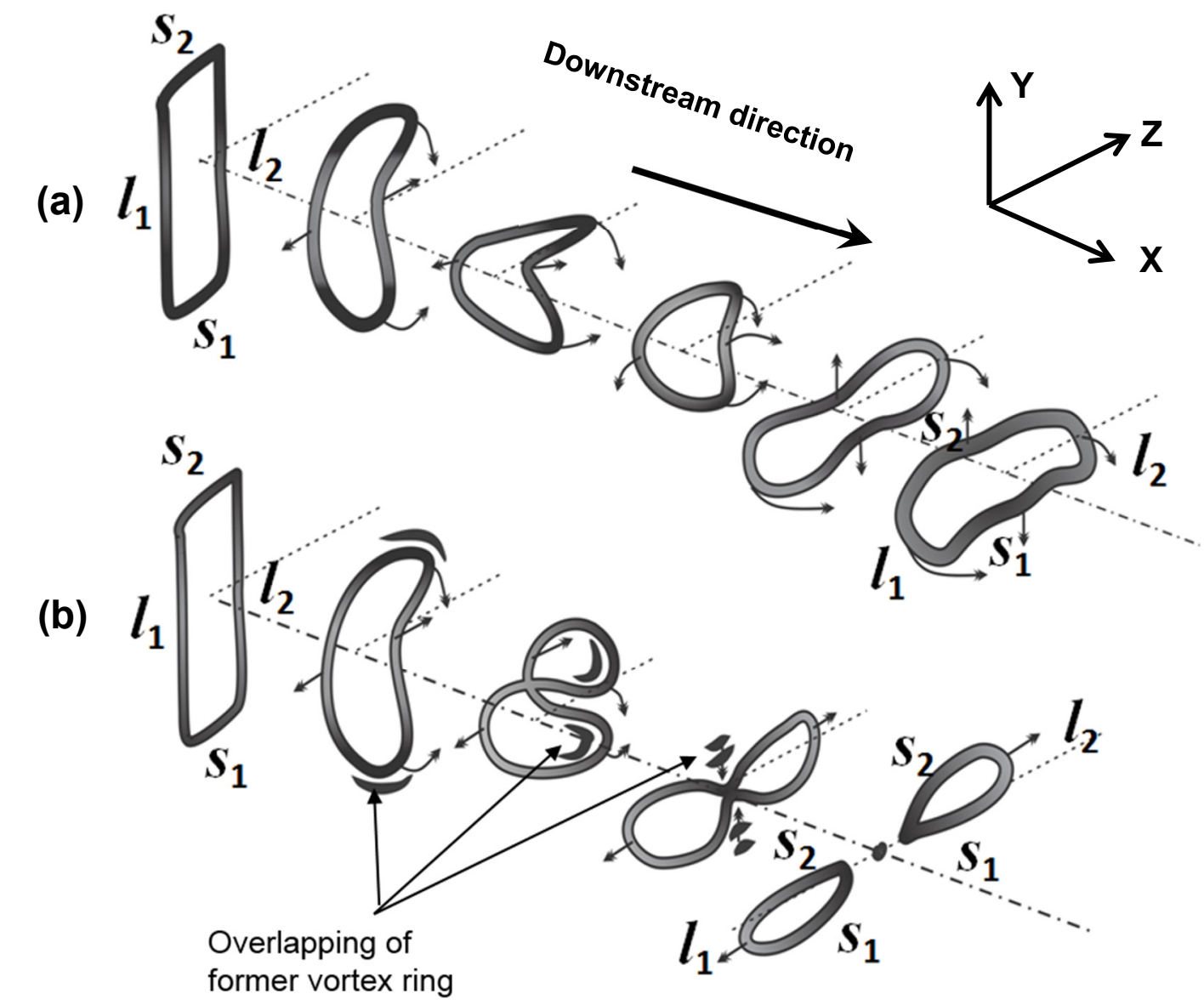

Figure 5.7: A schematic comparing the process of (a) axial switching and (b) bifurcation in rectangular synthetic jet. 


\subsection{Time-averaged flow field}

The velocity components measured using LDV along synthetic jet centerline is shown in Figure 5.8. The dark symbols represent the cases of axial switching or non-bifurcation vortex rings and the gray symbols represent the cases where bifurcation is obtained.

\subsubsection{Centerline velocity distribution}
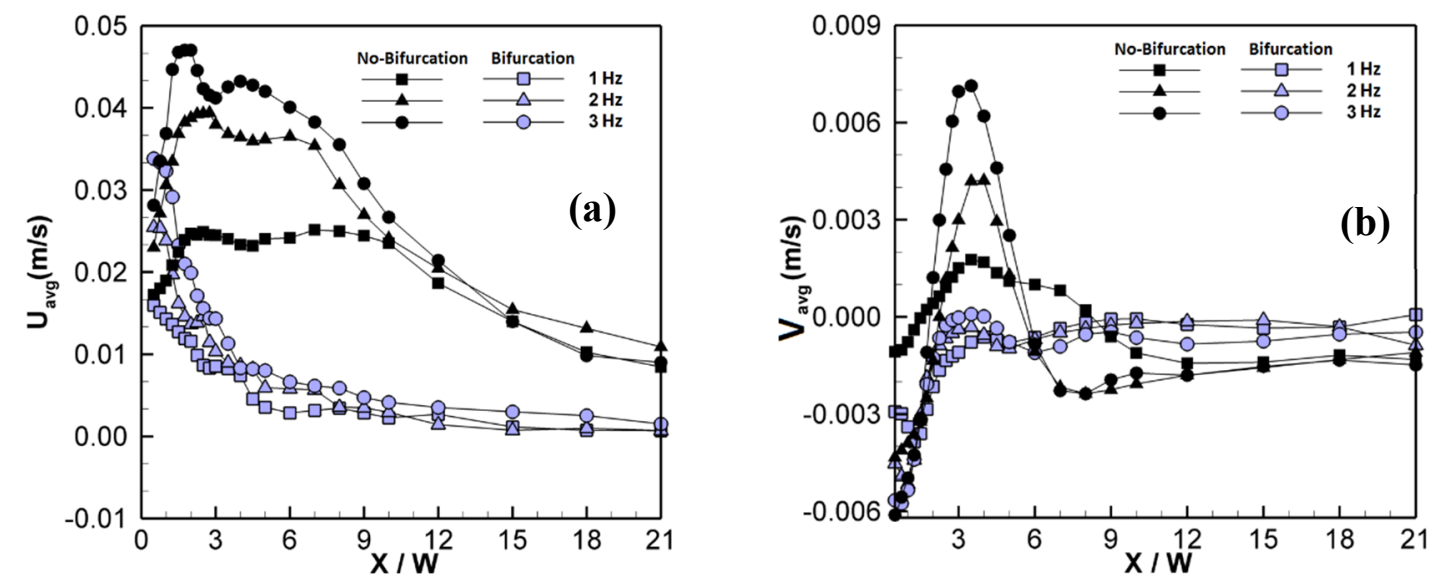

Figure 5.8: Time-averaged (a) streamwise velocity $\left(\mathrm{U}_{\text {avg }}\right)$ and $(\mathrm{b})$ cross-stream velocity $\left(\mathrm{V}_{\text {avg }}\right)$ along the synthetic jet centerline for bifurcation cases ((I), (II) and (III)) and for non-bifurcation cases ((IV), (V) and (VI)).

Figure 5.8 (a) presents the streamwise $\left(\mathrm{U}_{\text {avg }}\right)$ velocity and along the synthetic jet centerline for the bifurcation and non-bifurcation cases. The stream wise velocity component decreases rapidly from the orifice exit to $\mathrm{X} / \mathrm{W}=4$ in Figure 5.8 (a) for all the bifurcation cases. This is expected because during vortex reconnection the counter rotating vortices cancel each other vorticity, decreasing the time-averaged velocity in streamwise direction. After $\mathrm{X} / \mathrm{W}=4$ the velocity is negligible as the vortex ring splits and moves away from the synthetic jet centerline (see Figure 5.2). The non-bifurcating cases show a steep increase in velocity within the suction zone $(\mathrm{X} / \mathrm{W}<3)$ followed by a secondary peak. The undulation of velocity in the region between $\mathrm{X} / \mathrm{W}=4$ to 9 is related to the axial switching of vortex ring. During the process of axial switching, fluid along the synthetic jet centerline experiences periodic variation in its magnitude. 
The cross-stream velocity component $\left(\mathrm{V}_{\mathrm{avg}}\right)$ shown in Figure $5.8(\mathrm{~b})$ is negative (moving towards the synthetic jet centerline) for all the cases at the orifice exit. The crossstream velocity increases from the orifice exit and approaches to zero value near $\mathrm{X} / \mathrm{W}=3$ for all bifurcation cases whereas it is positive for all non-bifurcation cases with a peak at $\mathrm{X} / \mathrm{W}=4$. This indicates that the suction stroke adversely affects the vortex propagation in downstream direction for bifurcating cases in comparison to that of non-bifurcating cases. Since for bifurcation cases the suction stroke influences the flow field over a longer distance $(\mathrm{X} / \mathrm{W}=0$ to 3$)$, the process of vortex reconnection in $\mathrm{XY}$ plane is promoted (see Figure 5.6). At $\mathrm{X} / \mathrm{W}=3.5$ in Figure 5.4 (d), where the first axial switching is completed, the cross-stream velocity is positive and maximum for non-bifurcation cases but negative or zero for bifurcation cases as seen in Figure $5.8(\mathrm{~b})$. The positive value of cross-stream velocity at $\mathrm{X} / \mathrm{W}=3.5$ for non-bifurcation cases shows that the fluid at the vortex center moves away and prevent the possible reconnection of vortex tube for non-bifurcation cases. The velocity difference between the longer and shorter side of rectangular shape vortex ring is the prime source of first axial switching coupled with vortex reconnection and bifurcation in some conditions.

\subsubsection{Velocity distribution in transverse direction}

The time-averaged streamwise velocity $\left(\mathrm{U}_{\text {avg }}\right)$ and cross-stream velocity $\left(\mathrm{V}_{\text {avg }}\right)$ are shown in Figure 5.9 to Figure 5.12 for bifurcation and non-bifurcation cases in $\mathrm{X}$ and $\mathrm{Y}$-directions at downstream locations $\mathrm{X} / \mathrm{W}=1,2,3$ and 5 . The velocity is maximum along the synthetic jet centerline and decreases in Y-direction for bifurcation cases as shown Figure $5.9(\mathrm{a}-\mathrm{c})$. The velocity decreases rapidly and reaches to lower value between $\mathrm{Y} / \mathrm{B}=0.3$ to 0.4 , because in XY-plane the vortex rings moves towards the center (see Figure 5.2 (a) and (b)). Since bifurcation region lies between $\mathrm{X} / \mathrm{W}=2$ to 4 (see Figure 5.4 (b)), the velocity is not equal to zero in the near vicinity of synthetic jet centerline between $\mathrm{X} / \mathrm{W}=3$ and 5 in Figure 
5.9 (a-c). Significant amount of fluid drifts away from the synthetic jet centerline in XZplane due to vortex bifurcation. However, a small portion is left which moves along the synthetic jet centerline.

Figure 5.9 (i-iii), shows the velocity profile in Z-direction for bifurcation cases.

Comparison of velocity profile in Z-direction with velocity profile in Y-direction (Figure 5.9 (a-c)) show higher average velocity in Z-direction. This is due to the bifurcation in XZplane (see Figure 5.5). The overall magnitude of velocity reduces in X-direction while maintaining same overall trend.

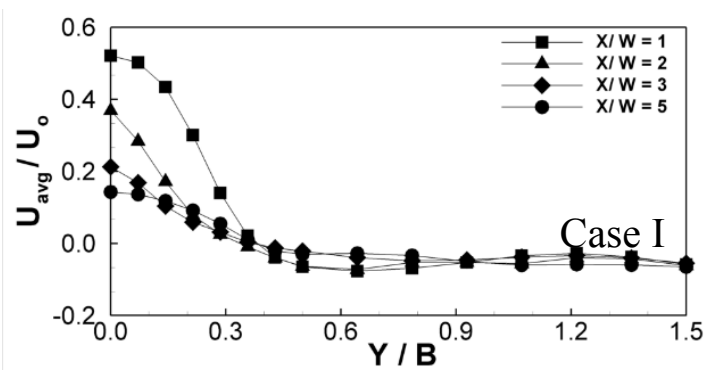

(a)

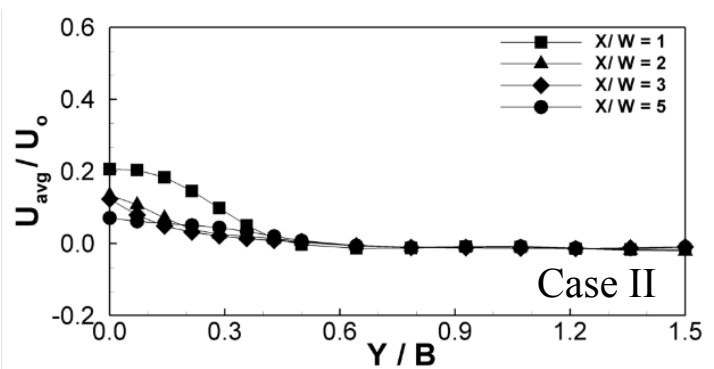

(b)



(c)

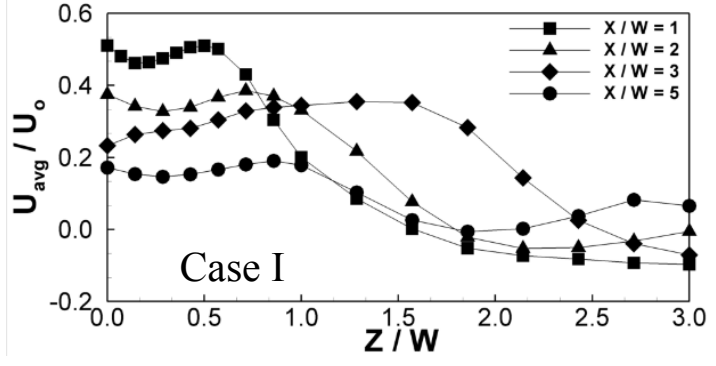

(i)

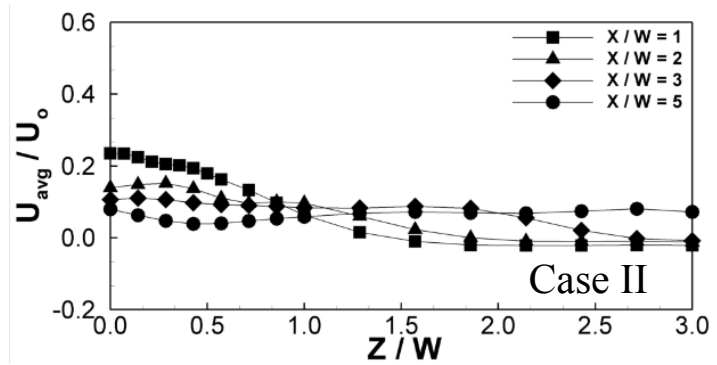

(ii)

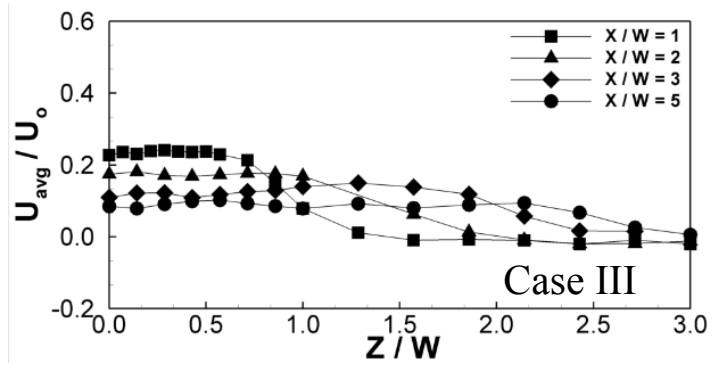

(iii)

Figure 5.9: Time-averaged streamwise velocity $\left(U_{\text {avg }}\right)$ profile normalised by $U_{0}$ for (a) Case $I$, (b) Case II and (c) Case III in Y-direction and (i) Case I, (ii) Case II and (iii) Case III in Z-direction at four downstream locations $(X / W=1,2,3$ and 5$)$. 


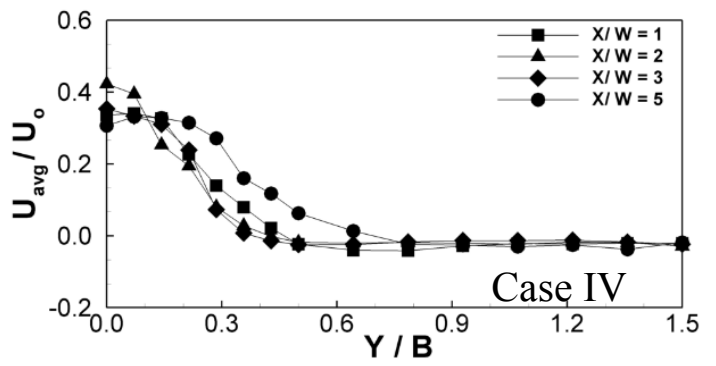

(a)

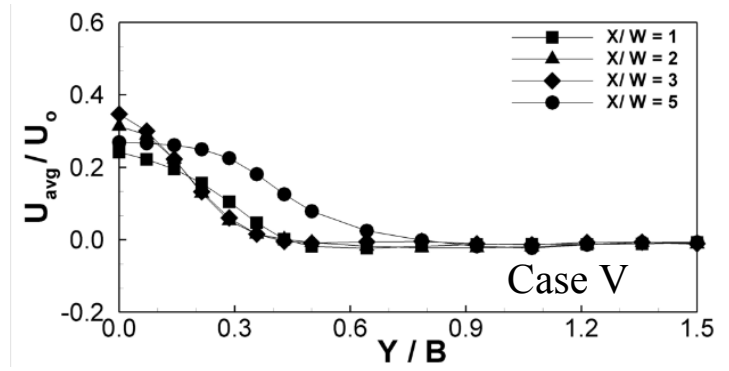

(b)



(c)



(i)



(ii)



(iii)

Figure 5.10: Time-averaged streamwise velocity ( $\left.U_{\text {avg }}\right)$ profile normalised by $U_{0}$ for (a) Case VI, (b) Case V and (c) Case VI in Y-direction and (i) Case VI, (ii) Case V and (iii) Case VI in Z-direction at four downstream locations $(\mathrm{X} / \mathrm{W}=1,2,3$ and 5$)$.

The velocity distribution in streamwise direction for non-bifurcating cases IV, V, and VI are shown in Figure 5.10. The streamwise velocity in Y-direction (major axis plane) follows similar trend for all three non-bifurcation cases with different slopes in between $\mathrm{Y} / \mathrm{B}=0$ to 0.6 in Figure 5.10(a-c). After $\mathrm{Y} / \mathrm{W}=1.2$, the velocity is negligible and no variation in velocity is observed which indicates that the spreading of vortex ring are limited within $\mathrm{Y} / \mathrm{W}=1.2$. The axial switching occurs between $\mathrm{X} / \mathrm{W}=1.8$ to 4.2 in Figure 5.4. Therefore, the velocity at $\mathrm{X} / \mathrm{W}=2$ and 3 is higher as compared to the slope at $\mathrm{X} / \mathrm{W}=$ 1 for non-bifurcation cases. Ho et al. (1987) reported that the large entrainment by elliptic jet of aspect ratio two is found in the minor axis plane (here $\mathrm{XZ}$ plane) of orifice and the 
entrainment was limited within the length of longer side of orifice in major axis plane (here $\mathrm{XY}$-plane) before $\mathrm{X} / \mathrm{W}<5$. Similar observation is seen by comparing the velocity distribution in $\mathrm{XY}$ and $\mathrm{XZ}$-plane in Figure 5.10. The velocity drops zero nearer to $\mathrm{Y} / \mathrm{B}=$ $0.5($ or $\mathrm{Y} / \mathrm{W}=1$ ) in $\mathrm{Y}$-direction but the velocity approaches to zero near $\mathrm{Z} / \mathrm{W}=1.5$ in $\mathrm{Z}$ direction.

The cross-stream velocity profile in $\mathrm{Y}$ and Z-direction for both bifurcation and nobifurcation cases have been presented in Figure 5.11 and Figure 5.12 respectively. If the measurement line is in XY-plane, the cross-stream velocity component indicates the velocity of fluid perpendicular to this plane. If the measurement line is in XZ-plane, the fluid moves away (+ value) or towards the synthetic jet centerline (- value).

Figure 5.11 shows overall higher magnitude of $\mathrm{V}_{\text {avg }}$ velocity in $\mathrm{XZ}$-plane compared to that in XY-plane. This observation relate to the occurrence of bifurcation in XZ-plane. The effect of bifurcation is higher near the synthetic jet orifice region $(\mathrm{X} / \mathrm{W}=1)$ leading to higher $\mathrm{V}_{\text {avg }}$ velocity. Figure 5.12 shows higher $\mathrm{V}_{\text {avg }}$ velocity in $\mathrm{XZ}$-plane compared to the XY-plane. However, the average velocity in XZ-plane for no-bifurcation case in Figure 5.12 is lower as compared to the bifurcation case in Figure 5.11.



(a)

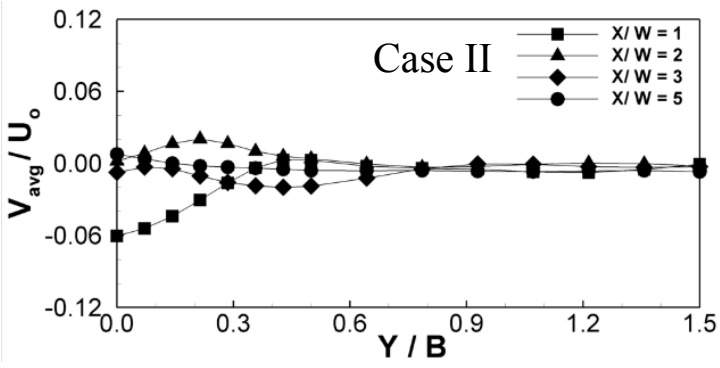



(b)

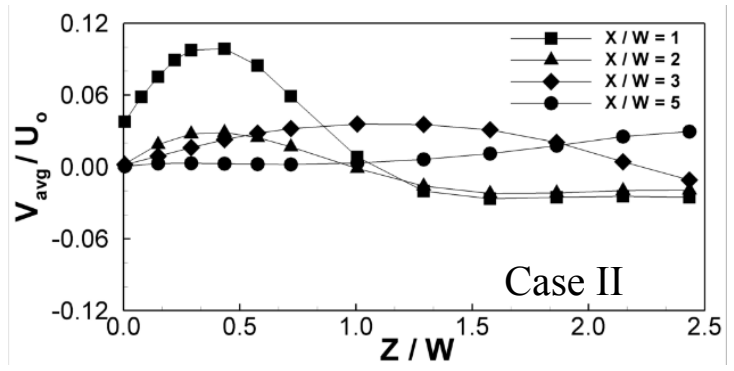


(c)

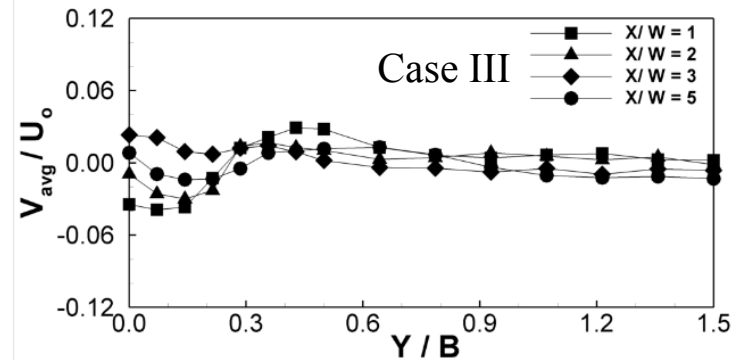

(e) (d)



(f)

Figure 5.11 Time-averaged cross-stream velocity ( $V_{\text {avg }}$ profile normalised by $U_{0}$ for (a) Case $I$, (b) Case II and (c) Case III in Y-direction and (i) Case I, (ii) Case II and (iii) Case III in Z-direction at four downstream locations $(\mathrm{X} / \mathrm{W}=1,2,3$ and 5).



(a)

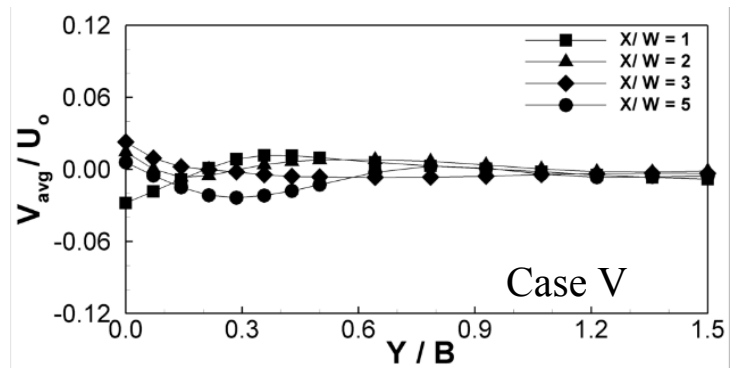

(c)

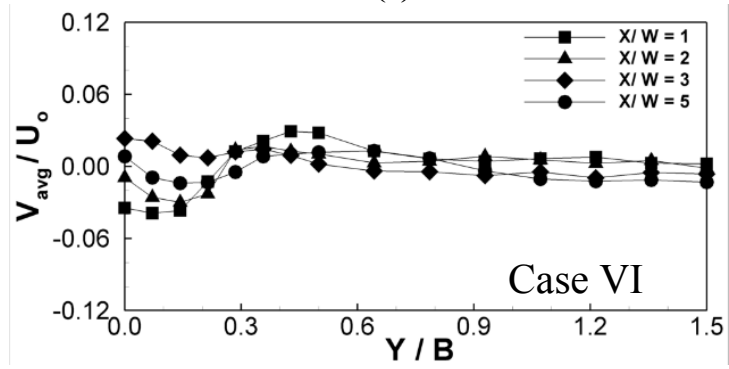

(e)

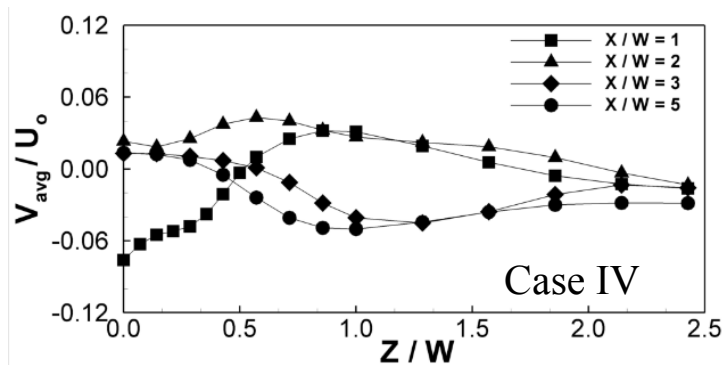

(b)

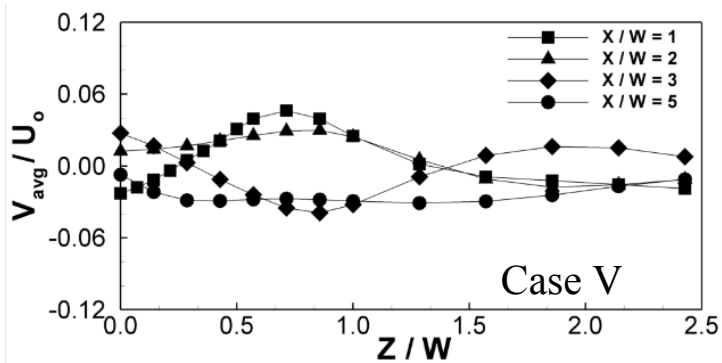

(d)

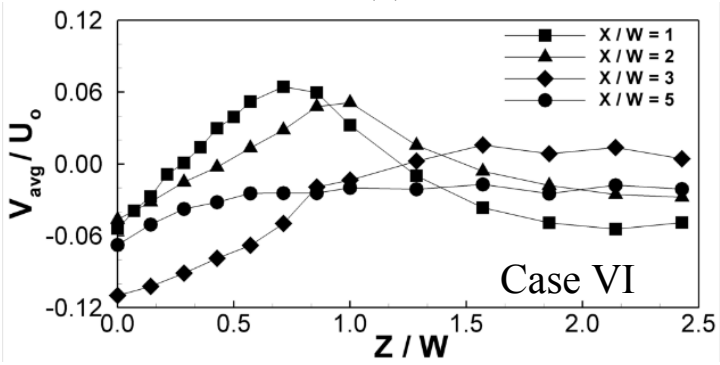

(f)

Figure 5.12: Time-averaged cross-stream velocity ( $\left.V_{\text {avg }}\right)$ profile normalised by $U_{0}$ for (a) Case III, (b) Case V and (c) Case VI in Y-direction and (i) Case III, (ii) Case V and (iii) Case VI in Z-direction at four downstream locations $(\mathrm{X} / \mathrm{W}=1,2,3$ and 5$)$. 


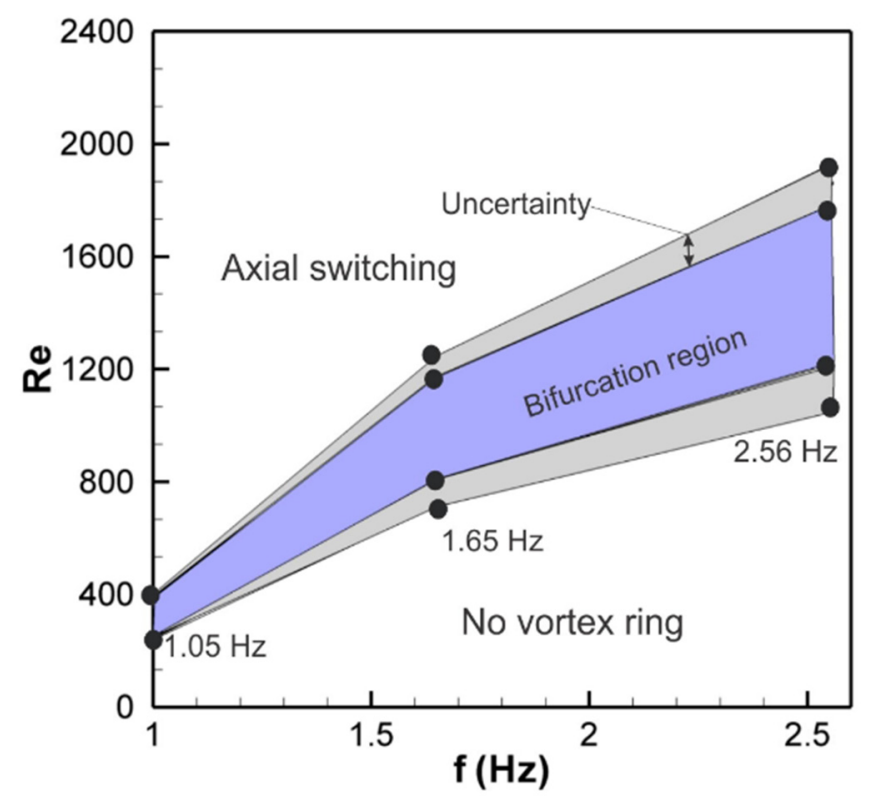

Figure 5.13: The range of Reynolds number for bifurcation observed from visualization images of synthetic jet of aspect ratio 2 .

The range of Reynolds number in which the rectangular shaped synthetic jet leads to bifurcation for orifice of aspect ratio two under the mentioned experimental conditions are shown in Figure 5.13 for actuation frequency $1.05 \mathrm{~Hz}, 1.65 \mathrm{~Hz}$ and at $2.65 \mathrm{~Hz}$ (rounded values are 1, 2 and $3 \mathrm{~Hz}$ ). At these three frequencies, the Reynolds number is varied by changing the magnitude of diaphragm displacement and the range in which vortex bifurcation occurs is reported. It has been observed that the range of Reynolds number for vortex bifurcation is sensitive to the actuation frequency. The range of Reynolds number in which the vortex ring bifurcates, increases with increasing the actuation frequency and no vortex splitting is observed in major axis plane $(\mathrm{XY})$ within the range shown in Figure 5.13. Below the shaded region, there is no distinct formation of vortex ring. In this region, the fluid ejected out from the orifice is sucked back partially or completely inside the cavity during following suction stroke. Above the shaded region, no distinct vortex splitting is observed and the formed vortex ring experiences the axial switching. 


\subsection{Closure}

The bifurcation in rectangular synthetic jet of aspect ratio two has been observed at 1, 2 and $3 \mathrm{~Hz}$ actuation frequency. The bifurcation and axial switching of leading vortex have been studied quantitatively and qualitatively for orifice of hydraulic diameter equal to 9.33 mm. It has been observed that the vortex splitting or bifurcation occurs in the minor axis plane of orifice perpendicular to the longer side of orifice. The divergence angle depends on the actuation frequency and average velocity of fluid expulsion through the orifice. The main source of bifurcation is variation in vortex tube cross-section area (due to differences in velocity) during the shear layer roll-up and leap forging near the orifice exit in major axis plane of orifice. For axial switching, the maximum variation in averaged velocity takes place in minor axis plane of orifice rather than major axis plane. It is also observed that the vortex ring experiences maximum of three axial-switching before breakup. 


\section{Chapter 6}

\section{Synthetic jet in crossflow}

In this chapter, a synthetic jet in crossflow having a circular orifice of $6 \mathrm{~mm}$ diameter and $6 \mathrm{~mm}$ thick has been investigated. The orifice is flush mounted on a torpedo shaped model, the detail of which is given in Chapter 3. The study in quiescent flow is initially carried out for two different diaphragm displacements $(1.5$ and $2 \mathrm{~mm})$ and four different actuation frequencies namely, 1, 2, 4, and $6 \mathrm{~Hz}$. The investigation is then carried out in crossflow for three different crossflow velocities $(0.072,0.2$ and $0.32 \mathrm{~m} / \mathrm{s}$ ) and the diaphragm displacement is kept constant at $2 \mathrm{~mm}$. Since, no flow is observed out of the orifice at an actuation frequency of $1 \mathrm{~Hz}$, the measurements have been undertaken for actuation frequencies of 2, 4, and $6 \mathrm{~Hz}$. The hydraulic diameter, Reynolds number and others parameters calculated using Equation 3.1 and 3.2 corresponding to various frequencies are listed in Table 6.1. The flow field is discussed using bulk-flow images acquired from the side and top of the test section as shown Figure 6.1 (b) giving qualitative information and the findings are supported quantitatively by the velocity measurements using Laser Doppler Velocimetry. The LIF images on centre plane of the synthetic jet are also captured in both quiescent and crossflow conditions. The orifice orientation and co-ordinate systems used for both conditions of flow (quiescent and crossflow) are shown in Figure 6.1. 


\subsection{Synthetic jet in quiescent flow}

Prior to the study of synthetic jet in crossflow, the behaviour of the same synthetic jet device is analysed in quiescent flow for two different diaphragm displacements and four different actuation frequencies. It is to be noted that for simplicity, the frequencies are given its nearest integer values though the actual values are fractional while discussing results. As mentioned earlier, the actuation frequencies and their corresponding non-dimensional numbers are tabulated in Table 6.1. A flow visualization study and measurement of timeaveraged flow variation along synthetic jet centerline is used to characterize the behaviour of synthetic jet.

Table 6.1: Experimental parameters to study the synthetic jet built in on torpedo shape model in quiescent flow.

\begin{tabular}{|c|c|c|c|c|c|c|c|}
\hline \multicolumn{2}{|c|}{ (f) Hz } & \multicolumn{3}{c|}{$\Delta=\mathbf{1 . 5} \mathbf{~ m m}$} & \multicolumn{3}{c|}{$\Delta=\mathbf{2 ~ m m}$} \\
\hline Actual & Round-off & Res & S & L/D & ResJ & S & L/D \\
\hline 1.23 & 1 & 323 & 8.5 & 4.56 & 425 & 8.3 & 6.08 \\
\hline 2.04 & 2 & 550 & 11 & 4.56 & 732 & 11 & 6.08 \\
\hline 3.92 & 4 & 1030 & 15 & 4.56 & 1372 & 15 & 6.08 \\
\hline 5.88 & 6 & 1544 & 18.3 & 4.56 & 2058 & 18.3 & 6.08 \\
\hline
\end{tabular}
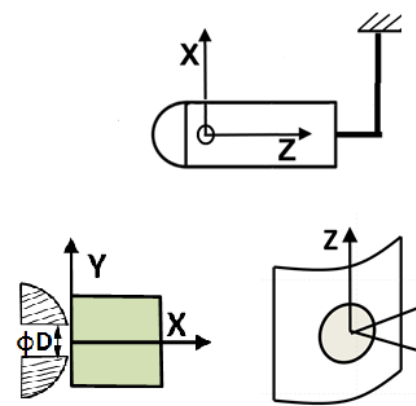

Image plane

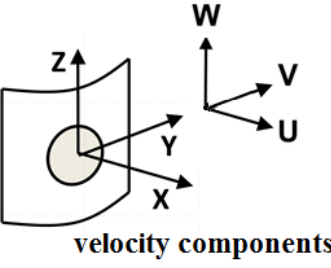

(a)


(b)

Figure 6.1: Schematic for axis notation and orifice orientation with imaging plane for torpedo shape model (a) quiescent flow set-up and (b) crossflow set-up. 


\subsubsection{Flow visualization}

The flow structures of synthetic jet for two different amplitudes of oscillation and four different frequencies has been illustrated using the visualization images taken on XY-plane bisecting the orifice diametrically have been shown in Figure 6.2.

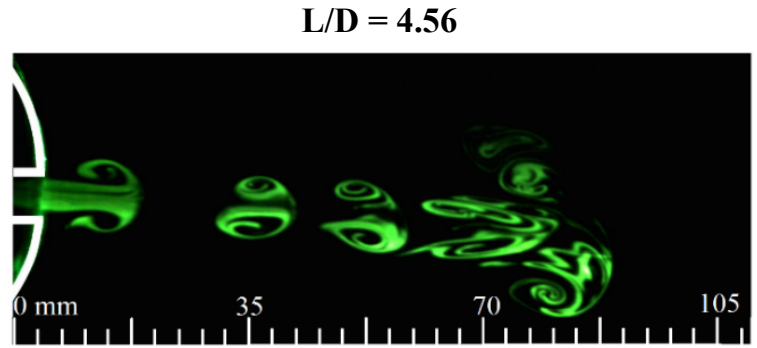

(a) $\Delta=1.5 \mathrm{~mm}, \mathbf{f}=1 \mathrm{~Hz}$

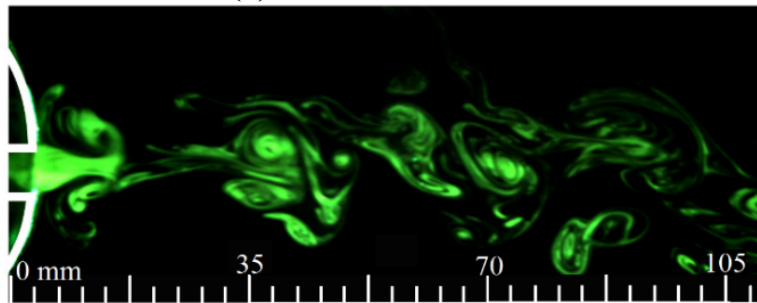

(c) $\Delta=1.5 \mathrm{~mm}, \mathrm{f}=2 \mathrm{~Hz}$



(e) $\Delta=1.5 \mathrm{~mm}, \mathrm{f}=4 \mathrm{~Hz}$

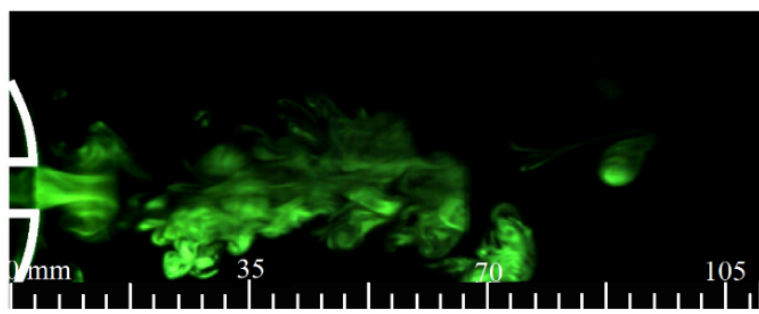

(g) $\Delta=1.5 \mathrm{~mm}, \mathbf{f}=6 \mathrm{~Hz}$



(b) $\Delta=2 \mathrm{~mm}, \mathbf{f}=1 \mathrm{~Hz}$

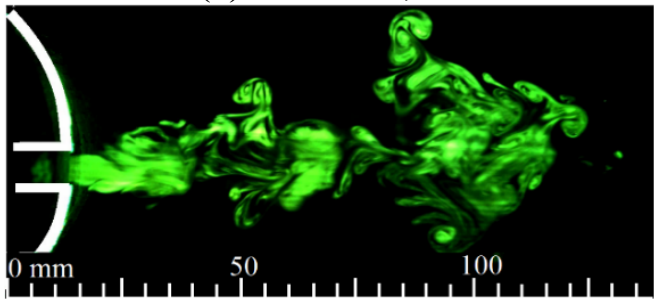

(d) $\Delta=2 \mathrm{~mm}, \mathrm{f}=2 \mathrm{~Hz}$



(f) $\Delta=2 \mathrm{~mm}, \mathrm{f}=4 \mathrm{~Hz}$

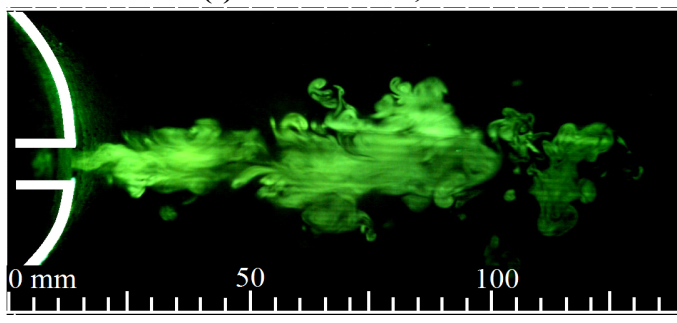

(h) $\Delta=2 \mathrm{~mm}, \mathrm{f}=6 \mathrm{~Hz}$

Figure 6.2: Sequence of LIF images at t/T $\sim 0.75$ for ((a) and (b)) at $1 \mathrm{~Hz}$, ((c) and (d)) at $2 \mathrm{~Hz},((\mathrm{e})$ and (f)) at $4 \mathrm{~Hz},((\mathrm{~g})$ and $(\mathrm{h}))$ at $6 \mathrm{~Hz}$ actuation frequency for diaphragm dispcement $(\Delta)$ equal to $1.5 \mathrm{~mm}$ and $2 \mathrm{~mm}$ respectively.

At an actuation frequency of $1 \mathrm{~Hz}$, the strength of vortices formed due to the rolling of shear layer coming out of the orifice is found to be weak with low vorticity content. The position of the velocity peak in the jet is governed by Stokes number, which is believed to 
affect the rolling of shear layer. A lower value of Stoke number restricts shear layer from rolling while a higher value promotes the formation of strong vortex rings (for detail see Appendix A.2.4). An increase in actuation frequency increases the amount of fluid expelled out of the orifice and consequently increases the jet velocity. This in turn increases the Stokes number and helps in enhancing the vortex roll up process. Close examination of the images for $\Delta=1.5 \mathrm{~mm}$ at different frequencies reveals that the strength of the vortex rings increases while the flow at far downstream becomes complex with increasing small scale structures. The formation of partial trailing jet is also clearly visible for an actuation frequency of $4 \mathrm{~Hz}$. With an increase in $\mathrm{L} / \mathrm{D}$ ratio for a diaphragm displacement, $\Delta=2 \mathrm{~mm}$, more elongated and stronger trailing jet has been observed to form as compared to a smaller diaphragm displacement, $\Delta=1.5 \mathrm{~mm}$. As frequency increases, increasingly stronger and longer trailing jet is seen for $\Delta=2 \mathrm{~mm}$ and has been demonstrated in Figure 6.2.

\subsubsection{Time-averaged velocity field}

The two different velocity components namely, streamwise velocity (in the direction of synthetic jet) and the cross-stream velocity have been measured using LDV for two amplitudes and four different actuation frequencies. The axial variation of the two components of velocity is shown along the centerline of synthetic jet in Figure 6.3. For both amplitudes, the streamwise velocity is observed to have a monotonic behaviour with the axial distance. As expected, the time-mean velocity in the near vicinity of orifice increases with increasing frequency. The velocity remains higher and almost at constant value until $\mathrm{X} / \mathrm{D} \sim 4$, followed by a rapid fall up to $\mathrm{X} / \mathrm{D} \sim 9$, and then decreases slowly with axial distance. This trend matches with the normal trend of velocity variation observed for synthetic jet in quiescent flow where the jet producing device is attached to one of its wall. The undulation of the streamwise velocity in the near field in Figure 6.3(b) at $2 \mathrm{~Hz}$ may be attributed to the leap forging motion of vortex rings as observed in the sequence of LIF 
images in Figure 6.2. The positive value of cross-stream velocity component (V) represents the fluid flow outwards from the jet centerline while negative value signifies the flow towards the centreline. The vortex rings at $\Delta=1.5 \mathrm{~mm}$ are found to be quite coherent with weak trailing jet. The coherent nature of the ring leads to monotonic distribution of crossstream velocity shown in Figure 6.3 (c). But a non-monotonic distribution in Figure 6.3 (d) for $\Delta=2 \mathrm{~mm}$ may be due to chaotic or probably turbulent nature of jet that has small scale structures in trailing jet as noticed in Figure 6.2.

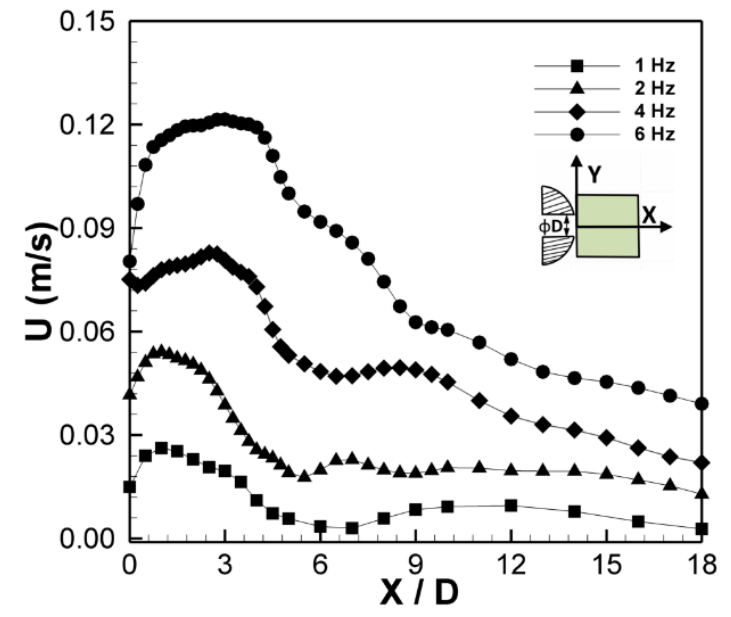

(a) $\Delta=1.5 \mathrm{~mm}, \mathrm{~L} / \mathrm{D}=4.56$



(c) $\Delta=1.5 \mathrm{~mm}, \mathrm{~L} / \mathrm{D}=\mathbf{4 . 5 6}$

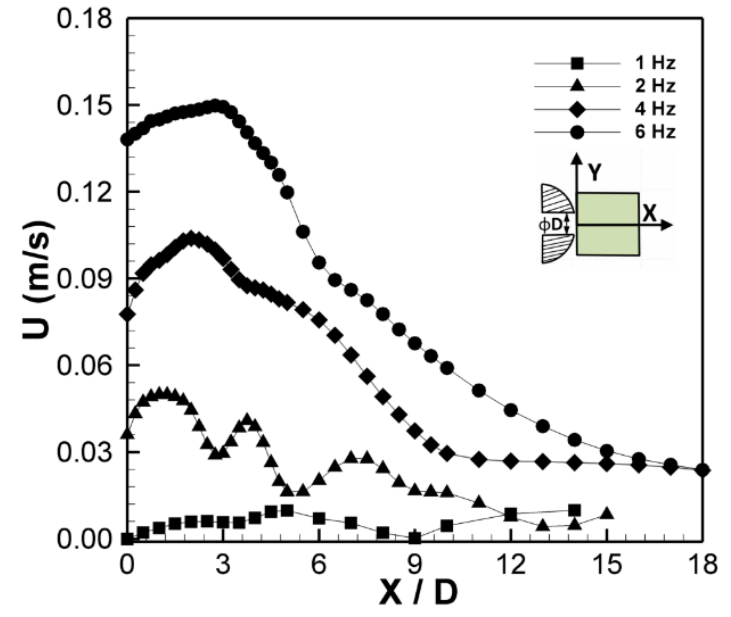

(b) $\Delta=2 \mathrm{~mm} \mathrm{~L} / \mathrm{D}=\mathbf{6 . 0 8}$

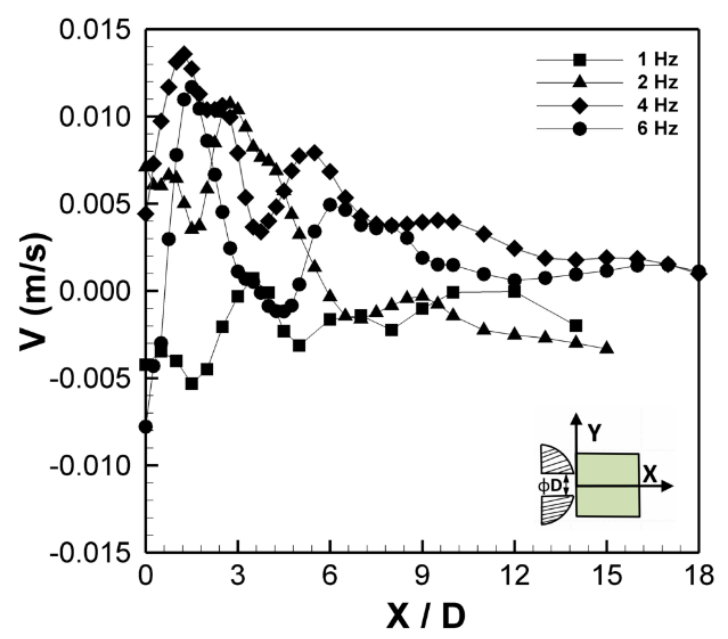

(d) $\Delta=2 \mathrm{~mm}, \mathrm{~L} / \mathrm{D}=\mathbf{6 . 0 8}$

Figure 6.3: Time averaged streamwise velocity profile (a) and (b) and cross-stream velocity profile (c) and (d) for $\Delta=1.5 \mathrm{~mm}$ and $\Delta=2 \mathrm{~mm}$ respectively along the synthetic jet centerline. 


\subsection{Synthetic jet in crossflow}

The experimental conditions to study the behaviour of synthetic jet in crossflow are tabulated in Table 6.2. The orifice position and the coordinate system are also shown in Figure 6.1(b). In this study, the velocity ratio (VR) is varied in the range of $0.34-4.77$, Reynolds number of synthetic jet for no crossflow condition $\left(\mathrm{U}_{\infty}=0\right)$ is set to three different values namely, 732, 1372, and 2058. The actuation frequency is also varied been 2 to $6 \mathrm{~Hz}$ depending on the crossflow velocity.

Table 6.2 Experimental parameters to study the synthetic jet built-in in torpedo shape model in crossflow

\begin{tabular}{|c|c|c|c|c|c|}
\hline \multirow{3}{*}{ Cases } & \multirow{2}{*}{$\mathbf{U}_{\infty}(\mathbf{m} / \mathbf{s})$} & \multicolumn{2}{|c|}{$\begin{array}{c}\text { Actuation frequency } \\
(\mathbf{f}) \mathbf{H z}\end{array}$} & $\begin{array}{c}\text { Reynolds } \\
\text { number (Ress) }\end{array}$ & $\begin{array}{c}\text { Velocity ratio } \\
\text { (VR) }\end{array}$ \\
\cline { 3 - 6 } & & actual & rounded & \\
\hline \multirow{3}{*}{1} & 0.072 & 2.04 & 2 & 732 & 1.69 \\
\cline { 2 - 6 } & 0.072 & 3.92 & 4 & 1372 & 3.18 \\
\cline { 2 - 6 } & 0.072 & 5.88 & 6 & 2058 & 4.77 \\
\hline \multirow{3}{*}{2} & 0.2 & 2.04 & 2 & 732 & 0.61 \\
\cline { 2 - 6 } & 0.2 & 3.92 & 4 & 1372 & 1.14 \\
\cline { 2 - 6 } & 0.2 & 5.88 & 6 & 2058 & 1.72 \\
\hline \multirow{3}{*}{3} & 0.32 & 2.04 & 2 & 732 & 0.34 \\
\cline { 2 - 6 } & 0.32 & 3.92 & 4 & 1372 & 0.64 \\
\cline { 2 - 6 } & 0.32 & 5.88 & 6 & 2058 & 0.95 \\
\hline
\end{tabular}

As discussed above while discussing jet in quiescent flow, it has been observed that higher diaphragm displacement contributes to stronger trailing jet. The secondary vortices or trailing jet of synthetic jet in crossflow induces streamwise tertiary vortices near the wall. In time-averaged sense, this vortices corresponding to the legs embedded in a boundary layer exhibit characteristics similar to a longitudinal vortex pair giving a common downwash. Since the higher displacement gives rich flow physics, a diaphragm displacement of $\Delta=2 \mathrm{~mm}$ is opted for the study of synthetic jet in crossflow. The corresponding $\mathrm{L} / \mathrm{D}$ ratio is 6.08 , which is on the higher side. The bulk flow visualization is 
carried out using incandescent light. Two different projected views; XZ (side view) and YZ (top view) are taken. Near the orifice, the LIF images are also captured on XZ-plane. Two components of time-averaged flow velocity is measured using LDV technique. The details regarding the experimental set-up and measurement technique has been discussed in Chapter 3.

\subsubsection{Flow visualization}

Both bulk flow visualization using color dye and LIF technique have used to capture complex flow structures. While bulk flow visualization gives the complete threedimensional picture of the flow field while LIF gives the sectional field of the threedimensional field. However, the LIF provides more distinct flow features in comparison to bulk flow visualization.

Since it is customary to compare the jet flow under no crossflow condition with the jet flow experiencing a crossflow, the visualization of synthetic jet in quiescent flow has been carried out. Figure 6.4 presents the bulk flow visualization of synthetic jet under no crossflow velocity for various actuation frequencies. The synthetic jet at $2 \mathrm{~Hz}$ depicted in Figure 6.4 (a) generates leading vortex with elongated weak trailing jet in comparison to 4 Hz shown in Figure 6.4 (b) where the leading vortex ring is followed by strong coherent trailing jet. At an actuation frequency of $6 \mathrm{~Hz}$ (Figure 6.4 (c)), synthetic jet reveals a complex trailing jet showing higher spreading in transverse direction. The approximate value of boundary layer thickness of the crossflow without synthetic jet in place is measured from the estimate of transverse distance where local velocity becomes $0.99 \mathrm{U}_{\infty}$. The boundary layer thickness so calculated is also termed here as the reference boundary layer. The values of boundary layer are 5, 3.5 and $2 \mathrm{~mm}$ respectively for crossflow velocities of $0.072,0.2$ and $0.32 \mathrm{~m} / \mathrm{s}$. The relative positions of the boundary layer are also shown using dotted lines above the model surface wherever necessary. It is to be noted that 
the images of side view (XZ-plane) and top view (YZ-plane) are not in phase and are at different instants of time because they are not taken simultaneously.
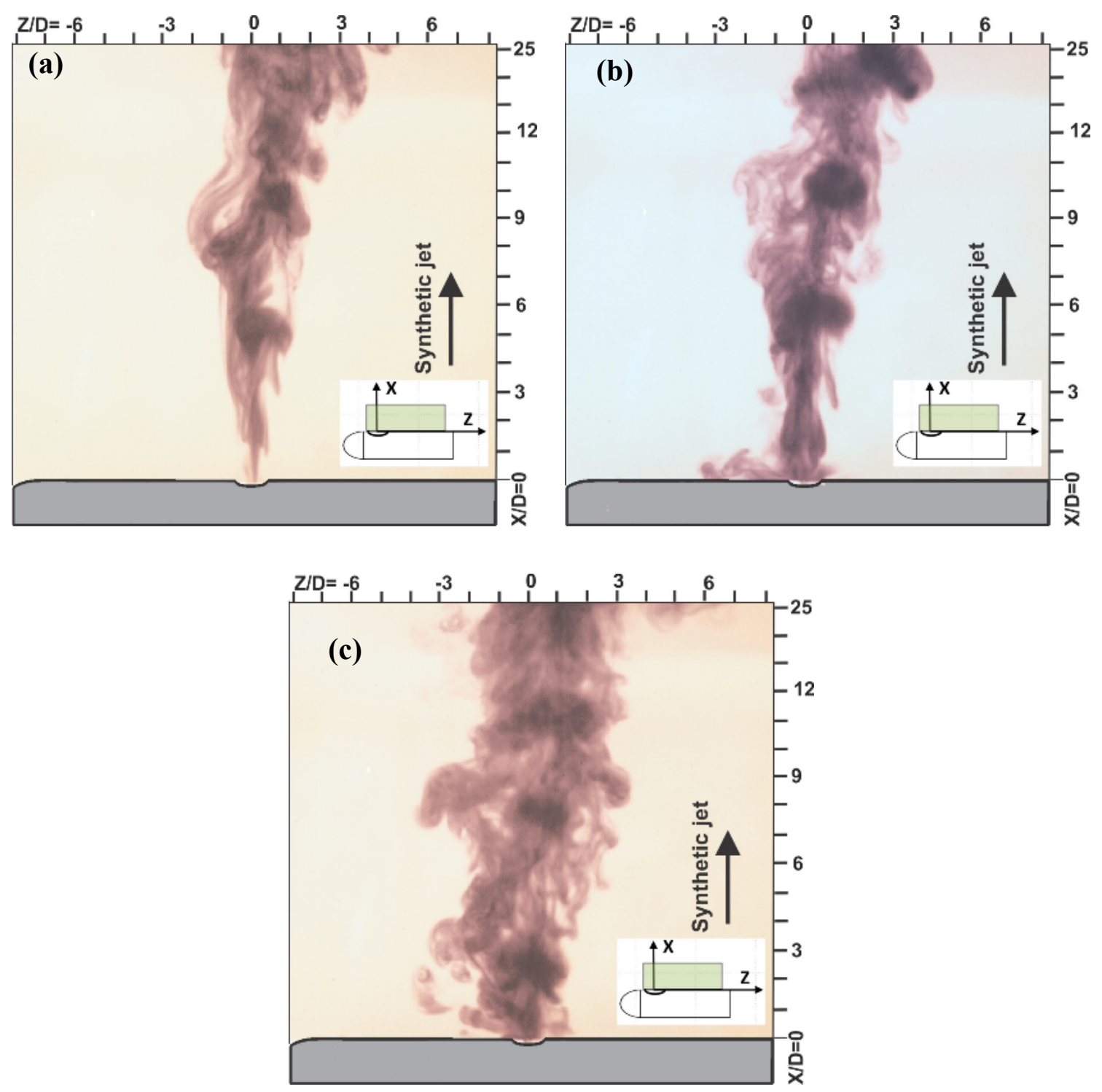

Figure 6.4: Bulk flow visualization of synthetic jet in side view at (a) $2 \mathrm{~Hz}$, (b) $4 \mathrm{~Hz}$ and (c) $6 \mathrm{~Hz}$ actuation frequency at crossflow velocity $\left(\mathrm{U}_{\infty}\right)=0$.

As discussed earlier that at lower frequency, the jet produced is weak. Close scrutiny of the synthetic jet at $2 \mathrm{~Hz}$ shows the formation of weak vortex ring and elongated trailing jet as seen in Figure 6.2 (d) and Figure 6.4 (a). The bulk flow images of synthetic jet at $2 \mathrm{~Hz}$ actuation frequency is shown in Figure 6.5 at different cross stream velocities $\left(\mathrm{U}_{\infty}\right)$ equal to $0.072,0.2$ and $0.32 \mathrm{~m} / \mathrm{s}$. For synthetic jet in crossflow, the flow structures can be grouped into three types such as: (a) hairpin vortices with maximum mass 
accumulated in its streamwise legs called hairpin vortex ring, (b) hairpin vortices where maximum mass accumulated at its head and is termed as stretched vortex ring and (c) the vortex ring making an angle to the direction of crossflow and experiences minimum or negligible distortion within boundary layer is referred as tilted vortex ring (see Figure 6.9). In Figure 6.5 (a), for $U_{\infty}=0.072 \mathrm{~m} / \mathrm{s}$, the shear layer rolls to form vortex rings which under the influence of crossflow becomes stretched in the streamwise direction. The flow in the near field of orifice experiences interaction between the vortex rings and the boundary layer causing an elongated leg between $\mathrm{Z} / \mathrm{D}=1$ and 5 . The distorted and stretched vortex ring is able to cross the reference boundary layer between axial distance, $\mathrm{Z} / \mathrm{D}=5$ and 10 and then travel in crossflow direction without any significant movement in the vertical direction, which is orthogonal to the crossflow direction. Chaudhry and Zhon (2012) reported similar flow structures (stretched but above the boundary layer). These types of structures generate strong fluid eruption from the wall and result in the generation of new vortices in wake of the jet particularly at higher velocity ratio known as induced streamwise vortices (see Figure 6.9(a)). In both views of visualization images, the two consecutive coherent structures are significantly far apart and consequently have a minimum interaction between them. The synthetic jet at same actuation frequency but at higher crossflow velocity $\left(\mathrm{U}_{\infty}=\right.$ $0.2 \mathrm{~m} / \mathrm{s}$ ) depicted in Figure 6.5 (b) experiences a higher stretching due to lower VR which reduces from 1.69 at $\mathrm{U}_{\infty}=0.072 \mathrm{~m} / \mathrm{s}$ to 0.61 at $\mathrm{U}_{\infty}=0.2 \mathrm{~m} / \mathrm{s}$. Because of the higher crossflow velocity, asymmetric vortex ring is formed at the exit of the orifice. The downstream part of vortex ring becomes quite stretched and travel downstream along the wall without experiencing any lift. The upstream portion of vortex ring pairs up with trailing jet of the preceding ring in the downstream and travel as a combined entity. When the crossflow is increased further to $\mathrm{U}_{\infty}=0.32 \mathrm{~m} / \mathrm{s}$, the VR decreases to 0.34 . As a consequence, the synthetic jet becomes highly stretched and leads to interconnected and 
closely spaced hairpin vortices with large number of streamwise streaks (see corresponding top view) in downstream. At this VR, the synthetic jet has transformed into a collection of highly stretched hairpin vortex rings, which remain close to the wall with major portion of the rings residing within the reference boundary layer.

In a quiescent flow, the leading vortex rings are observed to be closely packed for an actuation frequency of $4 \mathrm{~Hz}$ with coherent trailing jet near the orifice (see Figure 6.2(f)). However, in crossflow environment $\left(\mathrm{U}_{\infty}=0.072 \mathrm{~m} / \mathrm{s}\right)$, the velocity and vorticity content of leading vortex ring is seen to be higher than the trailing jet. The difference in velocity between the leading vortex rings and trailing jets leads to two different trajectories under the influence of crossflow velocity as shown in Figure 6.6 (a). The probable reason for such a flow structures are the different VR experienced by leading and trailing jets because of the unequal self-induced velocities. As discussed earlier, the velocity of leading vortex rings are more than that of the trailing jets causing two different VRs. The higher velocity of leading vortex rings leads to the trajectory caused by the higher elevation angle at the orifice exit than that of trailing jet indicated by dotted lines. The leading vortex rings are well separated and distinguishable in comparison to the synthetic jet in quiescent flow condition. This is because of the fact that the separation of the higher velocity leading vortex rings and the trailing jets does not allow the interaction between the two sets of jets. On the other hand, the interaction of leading and trailing jets has been observed in the far field and results in the loss of the coherency of the jet structures. The trailing jets are also found to be interlinked because of their low convection velocity and interact with each other in downstream (Figure 6.6 (a)). The entire jet travels in the crossflow direction but remains above the reference boundary layer. An increase in crossflow velocity to $\mathrm{U}_{\infty}=0.2$ $\mathrm{m} / \mathrm{s}$, the trailing jets become weak as large amount of fluid gets accumulated in the leading region of the vortex lifting the vortex structures upwards. However, they remains partially 
within the reference boundary layer (side view in Figure 6.6 (b)). These stretched vortex ring structures are the intermediate forms of hairpin and tilted vortex rings. The stretched vortex rings differ from hairpin vortex rings in the sense that maximum amount of ejected fluid having higher vorticity is accumulated in the head region of the vortex rather than in counter rotating vortex legs (Chaudhry and Zhong, 2014). The flow structures developed at an actuation frequency of $4 \mathrm{~Hz}$ and crossflow velocity of $0.32 \mathrm{~m} / \mathrm{s}$ depicted in Figure 6.6 (c) is almost similar to the flow structures shown in Figure 6.5 (c) where the actuation frequency and crossflow velocity is $2 \mathrm{~Hz}$ and $0.32 \mathrm{~m} / \mathrm{s}$ respectively. The number of vortex rings within equal axial length of the flow domain at $4 \mathrm{~Hz}$ actuation frequency is more than that at $2 \mathrm{~Hz}$ for equal crossflow velocity thus indicating lower interaction of two consecutive vortex rings for $2 \mathrm{~Hz}$.

The bulk flow visualization images taken for actuation frequency of $6 \mathrm{~Hz}$ are shown in Figure 6.7 at three crossflow velocities. For a crossflow velocity of $0.072 \mathrm{~m} / \mathrm{s}$, the elevation angle and penetration depth at $6 \mathrm{~Hz}$ is much higher in comparison to those at 4 $\mathrm{Hz}$ as shown in Figure 6.7 (a). The other significant difference between two flow structures at two frequencies is that the leading vortex rings and trailing jet interacts due to lower difference in their ejection velocity giving a sparsely distributed flow structures. 
Side view


(a) $\mathrm{U}_{\infty}=\mathbf{0 . 0 7 2} \mathrm{m} / \mathrm{s}, \mathrm{VR}=\mathbf{1 . 6 9}$

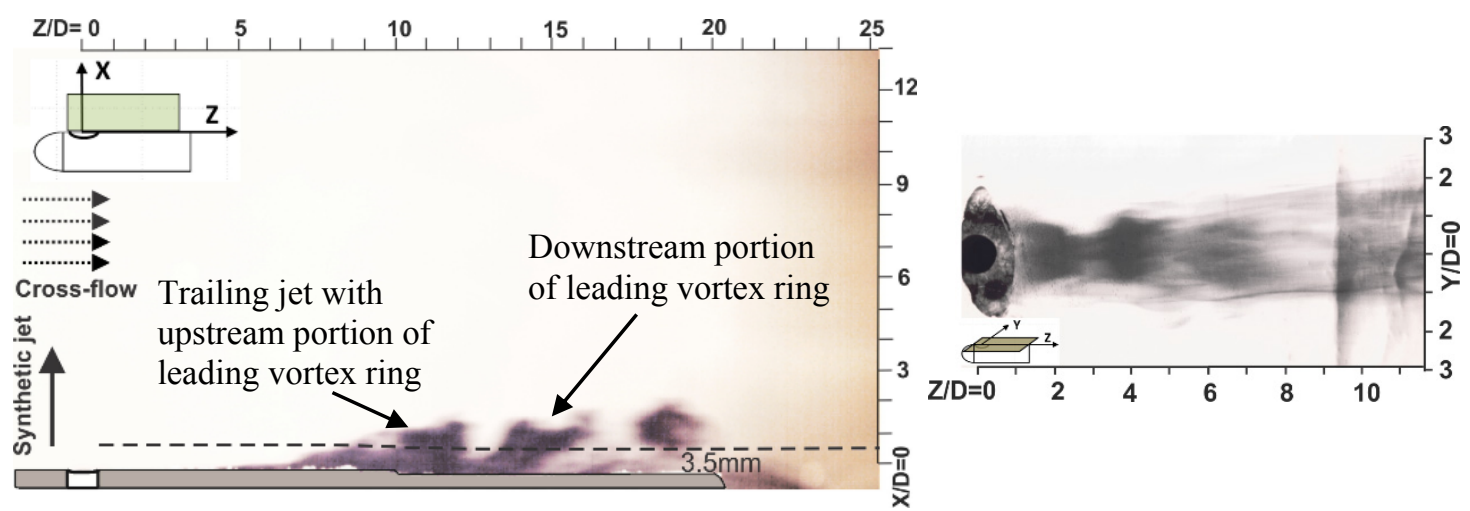

(b) $\mathrm{U}_{\infty}=0.2 \mathrm{~m} / \mathrm{s}, \mathrm{VR}=\mathbf{0 . 6 1}$

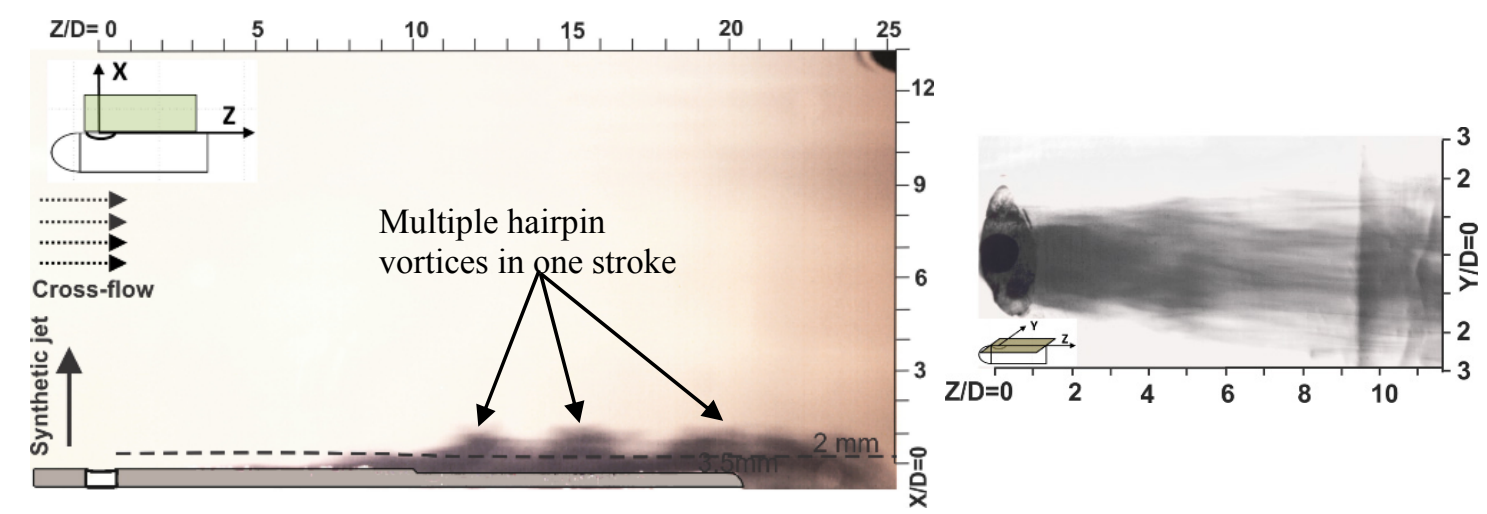

(c) $\mathrm{U}_{\infty}=0.32 \mathrm{~m} / \mathrm{s}, \mathrm{VR}=\mathbf{0 . 3 4}$

Figure 6.5: Bulk flow visualization of synthetic jet from side and top at crossflow velocity (a) 0.072 $\mathrm{m} / \mathrm{s}$ (b) $0.2 \mathrm{~m} / \mathrm{s}$ and (c) $0.32 \mathrm{~m} / \mathrm{s}$ at $2 \mathrm{~Hz}$ actuation frequency. (* The dashed line is approximate boundary layer thickness with synthetic jet at $0 \mathrm{~Hz}$ actuation frequency). 




(a) $\mathrm{U}_{\infty}=\mathbf{0 . 0 7 2} \mathrm{m} / \mathrm{s}, \mathrm{VR}=3.18$

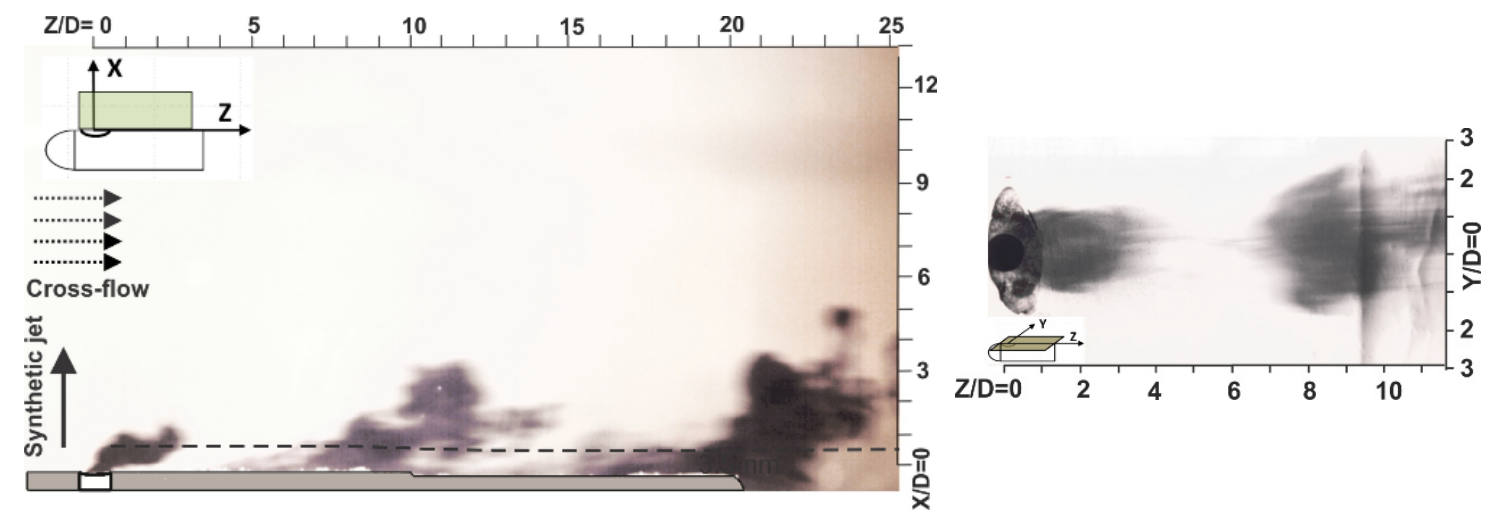

(b) $\mathrm{U}_{\infty}=0.2 \mathrm{~m} / \mathrm{s}, \mathrm{VR}=1.14$

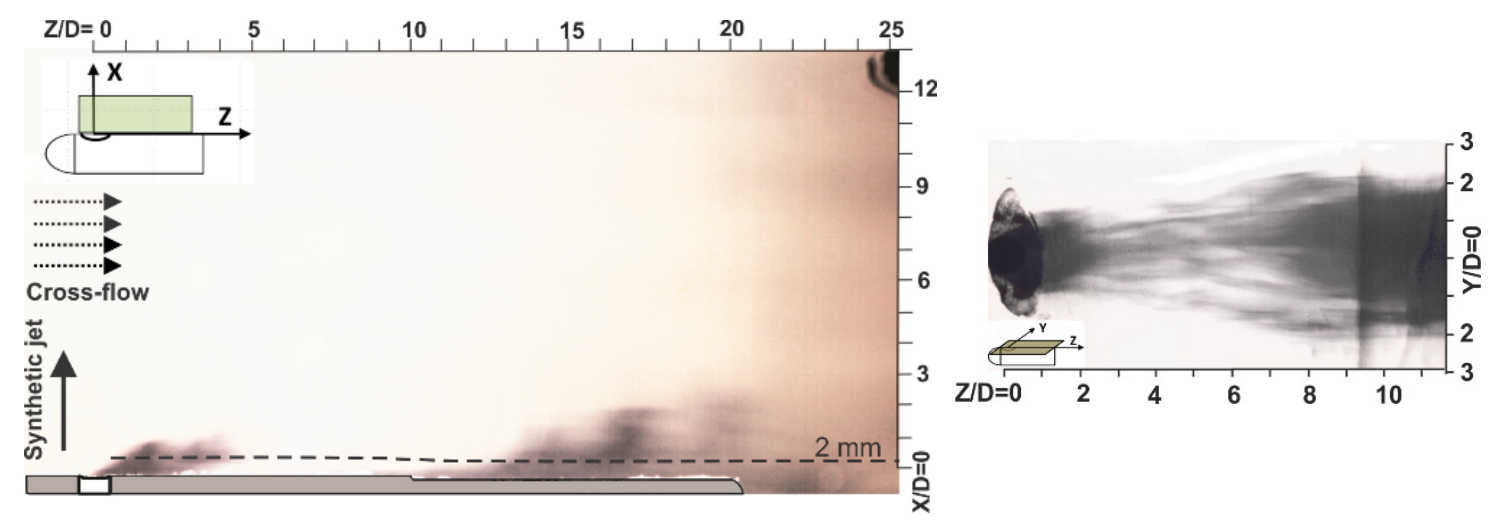

(c) $\mathrm{U}_{\infty}=0.32 \mathrm{~m} / \mathrm{s}, \mathrm{VR}=\mathbf{0 . 6 4}$

Figure 6.6: Bulk flow visualization of synthetic jet from side and top at crossflow velocity (a) 0.072 $\mathrm{m} / \mathrm{s}$ (b) $0.2 \mathrm{~m} / \mathrm{s}$ and (c) $0.32 \mathrm{~m} / \mathrm{s}$ at $4 \mathrm{~Hz}$ actuation frequency. (* The dashed line is approximate boundary layer thickness with synthetic jet at $0 \mathrm{~Hz}$ actuation frequency). 




Top view

(a) $\mathrm{U}_{\infty}=\mathbf{0 . 0 7 2} \mathrm{m} / \mathrm{s}, \mathrm{VR}=4.77$
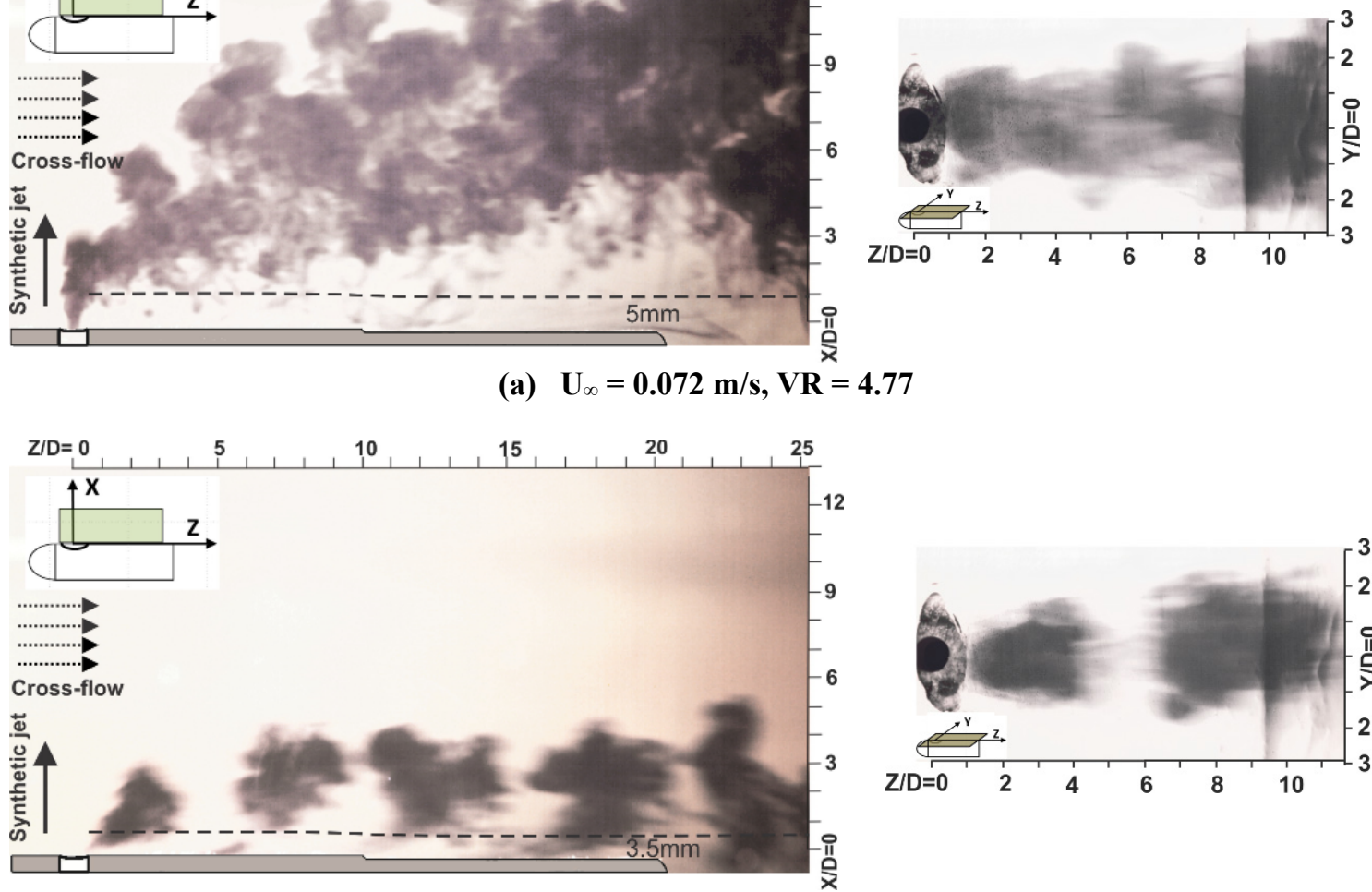

(b) $\mathrm{U}_{\infty}=0.2 \mathrm{~m} / \mathrm{s}, \mathrm{VR}=1.72$


(c) $\mathrm{U}_{\infty}=0.32 \mathrm{~m} / \mathrm{s}, \mathrm{VR}=\mathbf{0 . 9 5}$

Figure 6.7: Bulk flow visualization of synthetic jet from side and top at crossflow velocity (a) 0.072 $\mathrm{m} / \mathrm{s}$ (b) $0.2 \mathrm{~m} / \mathrm{s}$ and (c) $0.32 \mathrm{~m} / \mathrm{s}$ at $6 \mathrm{~Hz}$ actuation frequency. (* The dashed line is approximate boundary layer thickness with synthetic jet at $0 \mathrm{~Hz}$ actuation frequency). 
t/T $\sim 0.25$

$\mathrm{t} / \mathrm{T} \sim 0.5$

$\mathbf{t} / \mathbf{T} \sim \mathbf{0 . 7 5}$

$\mathbf{t} / \mathbf{T} \sim \mathbf{1 . 0}$
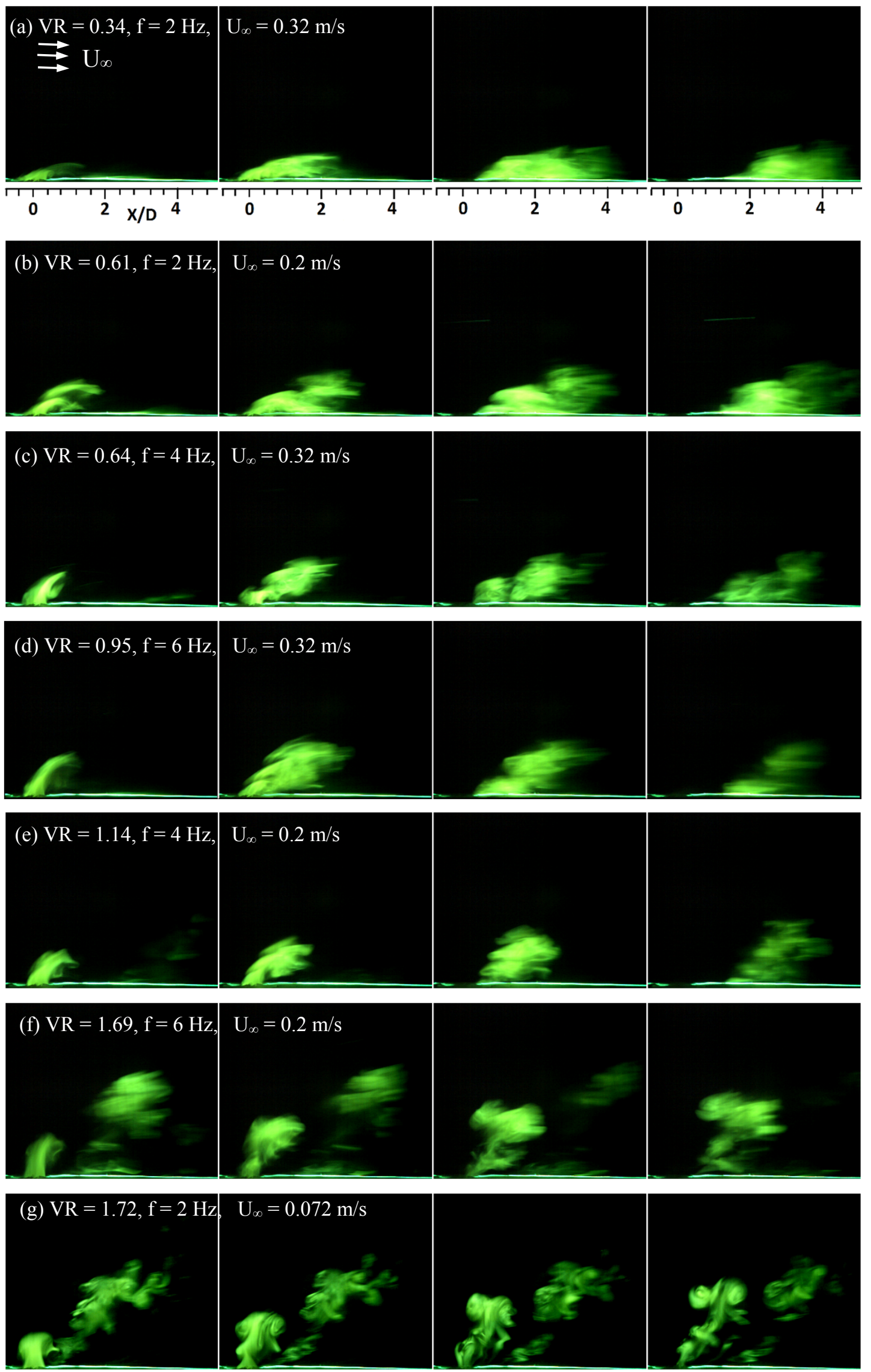

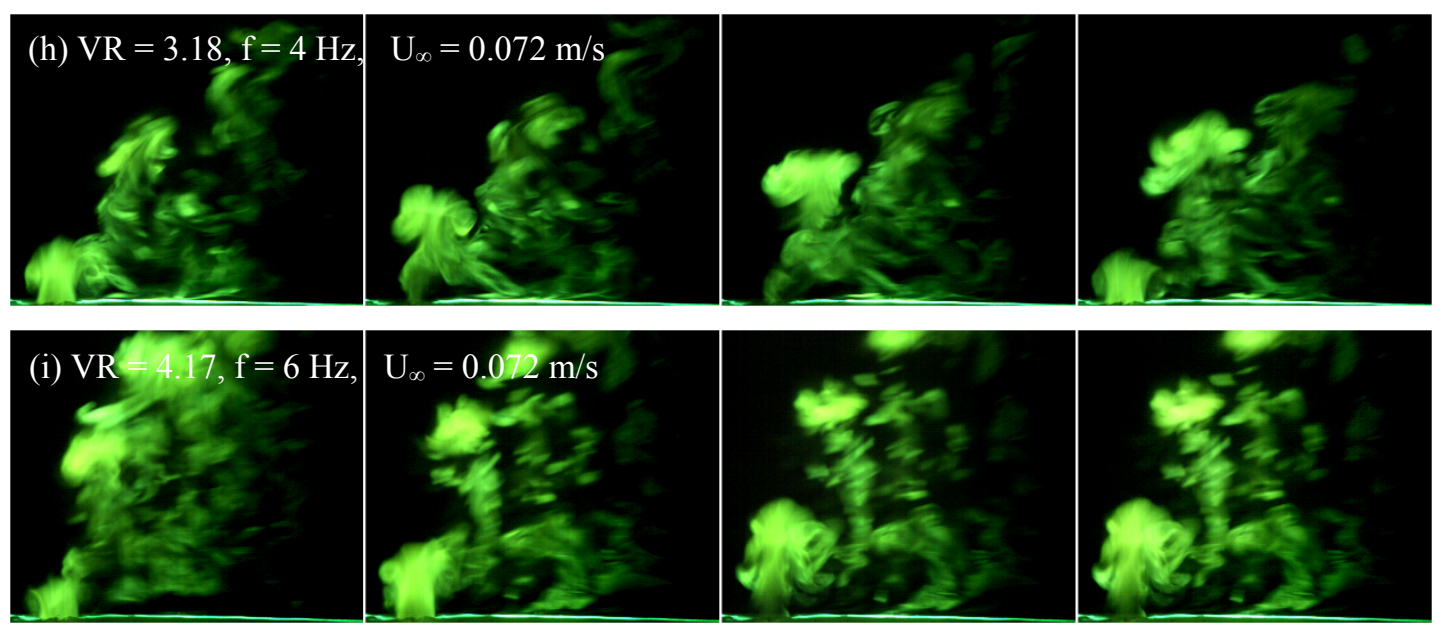

Figure 6.8: At crossflow velocity $0.072 \mathrm{~m} / \mathrm{s}, 0.2 \mathrm{~m} / \mathrm{s}$ and $0.32 \mathrm{~m} / \mathrm{s}$, the sequence of LIF images at actuation frequency 2,4 , and $6 \mathrm{~Hz}$ are arranged in ascending order of velocity ratio. The images are positioned at time stamp of $t / T \sim 0.25, t / T \sim 0.5, t / T \sim 0.75$ and $t / T \sim 1$ from left to right for each case.

The flow trajectories and evolution of vortex rings at $6 \mathrm{~Hz}$ in Figure 6.7 (b) for a velocity of $0.2 \mathrm{~m} / \mathrm{s}$ and Figure 6.7 (c) for a velocity of $0.32 \mathrm{~m} / \mathrm{s}$ closely resemble with those shown in Figure 6.5 (a) at $2 \mathrm{~Hz}$ (and velocity of $0.072 \mathrm{~m} / \mathrm{s}$ ) and Figure 6.6 (b) at $4 \mathrm{~Hz}$ (and velocity of $0.2 \mathrm{~m} / \mathrm{s}$ ) respectively. The probable reason for this behaviour is due to close match of VRs between the two different cases having different actuation frequencies. Analysis of the flow structures of visualization images at different frequencies and crossflow velocities reveals that the flow structures and evolution of synthetic jet in crossflow primarily depend on resulting VR which in turn depends on both the actuation frequency and crossflow velocity. For a given VR, Milanovic and Zaman (2005) found that the penetration depth collapses to a single point when scaled with the ratio of square of crossflow velocity to square of synthetic jet velocity.

It has been discussed earlier that the LIF images give a sectional view of the bulk flow field. Consequently, it provides better and distinct flow features compared to the bulk dye visualization where a projection of the entire three-dimensional integrated images in the direction of projection is taken thus eliminating the information on a particular plane. It is also noticed that VR is the most significant parameter affecting the jet flow when 
experiencing the crossflow. Therefore, the LIF flow visualization on XZ-plane cutting the orifice diametrically is aimed at the systematic comparison of the flow structures with increasing VR. At the lowest VR $(0.34$ at $2 \mathrm{~Hz})$, the hairpin vortices are found to form which is clear from Figure 6.8 (a). As mentioned earlier, asymmetric vortex rings are formed when the synthetic jet experiences a crossflow. Since the VR is quite low, the upstream part of the rings being weaker gets stretched significantly and made an envelope with the downstream part of the vortex. It is also expected that the jet formed in quiescent flow is quite elongated because of the formation of trailing jet at higher L/D ratio. Under the action of crossflow, more stretching of the structure transform it into a stretched vortex ring at $t / T=1$. Since the imaging plane bisects the hairpin vortices and shows only a slice in XZ-plane, both the legs of stretched vortices are not visible. Therefore, only the leading vortex along with thin shear layer upstream is captured by LIF technique. On the other hand, the sequence of bulk flow visualization (not shown here) can reveal phenomenon occurring out of the sectional plane of LIF. In the bulk flow visualization breakage of the structures has been observed in the downstream in Figure 6.5 (c). A higher value of stroke length $(\mathrm{L} / \mathrm{D}=6.08)$ and lower value of VR $(0.34)$ keep the vortices attached to the wall and the non-linear interactions between the boundary layer and the stretched vortices cause the structures to break after traveling a considerable distance downstream. Similar trend is also reported by Chaudhry and Zhong (2014) in case of stretched vortex ring at $\mathrm{L} / \mathrm{D}=6.67$ for circular orifice. It has been observed that at low VR, the synthetic jet remains within the boundary layer. Therefore, synthetic jet at low VR may be effective in controlling the boundary layer (Jabbal and Zhong, 2008). The difference in the structures between VR = 0.61 (Figure $6.8(\mathrm{~b})$ ) and VR $=0.34$ exists only in the near orifice field. The upstream part of vortex ring being weaker is swept away faster than the downstream part of the ring and rides over the downstream part. While traveling downstream, they also get stretched but 
remain close to the wall. The flow structures change significantly within the range of VR $(0.64,0.95$ and 1.14$)$. The vortex rings are observed to penetrate more compared to $\mathrm{VR}=0.34$ and 0.61 in the vertical direction before being convected in the crossflow direction. Unlike low VR cases $(\mathrm{VR}=0.34$ and 0.61$)$, the jet does not bend much but stretching of the jet is quite distinct. The trailing jet behind the tilted and distorted leading vortex ring without any distinct breakages are observed for $\mathrm{VR}=0.64$ to 1.14 . In this range of VR, jet has low Reynolds number but high Stroke length. Also the leading vortex ring is followed by strong trailing jet for a longer duration.

Further, at VR equal to 1.69 in Figure 6.8 (f), the flow structure differs significantly. Due to higher momentum of synthetic jet in comparison to crossflow, the leading vortex ring penetrates much well above the boundary layer and travel downstream. The trailing jet being weaker diffuses very quickly. When the VR is increased further (1.72, 3.18 and 4.17), the jet penetrates deep into the core of the crossflow without bending much in the flow direction (Figure $6.8(\mathrm{~g}-\mathrm{i})$ ). The tilted vortex rings experience no substantial stretching or distortion. The formation of long trailing jet or secondary vortices with multiple scales at $\mathrm{VR}=3.18$ and 4.77 confirms the incoherent nature of the trailing jet. For this higher VR, the leading vortex ring having higher self-induced velocity follows a trajectory different from the trailing jet covering the entire span between the trajectory of leading vortices and the wall.

The above analysis of flow visualization images and the studies available in the literature converge to a conclusion that the vortices or vortex rings in synthetic jet can be classified into two major groups: (a) stretched vortex ring and (b) tilted and distorted vortex ring. Stretched and elongated leading vortex ring, which occurs at low VR and remains attached to wall results in the formation of a hairpin structure or transformed into a stretched vortex ring. The basic difference between hairpin vortex ring and stretched vortex ring lies 
in the location of maximum vorticity as shown in Figure 6.9. If the legs of hairpin structures contain maximum amount of vorticity, they are known as hairpin vortices. However, if the maximum amount of vorticity is concentrated at the upward-lifted head region and gets stretched by the crossflow because of being partially above the boundary layer, it is called a stretched vortex ring. In case of tilted vortex ring, the leading vortex ring detached itself from wall and penetrates into the core crossflow due to higher momentum and induced velocity. If the trailing jet gets stretched in the form of hairpin structure behind the tilted vortex ring then it is seen to shed itself as coherent structures. The counter rotating legs of stretched hairpin vortices in Figure 6.9 (a) or the counter rotating stretched trailing jet in Figure 6.9 (b) are expected to induce two streamwise vortices beneath them because of the presence of wall. The interaction between the vortices of counter rotating legs with induced streamwise vortices draws higher momentum fluid near the wall from core crossflow and redistributes the vorticity content of the boundary layer. Since at low VR the maximum portion of the length of hairpin legs are close to the wall, they create a downwash bringing high momentum fluid from core flow towards the wall and thus increase the local shear stress.

It is also expected that because of the downwash as discussed in earlier paragraph, two streaks of walls shear stress is formed beneath the induced streamwise vortices as shown in Figure 6.9. The other vortices are also formed at upstream side of the orifice and are termed as upstream vortices or horse-shoe vortices (Wen and Tang, 2012). The magnitude and time-scale of upstream vortices are expected to depend on both VR and ejection period of synthetic jet. 


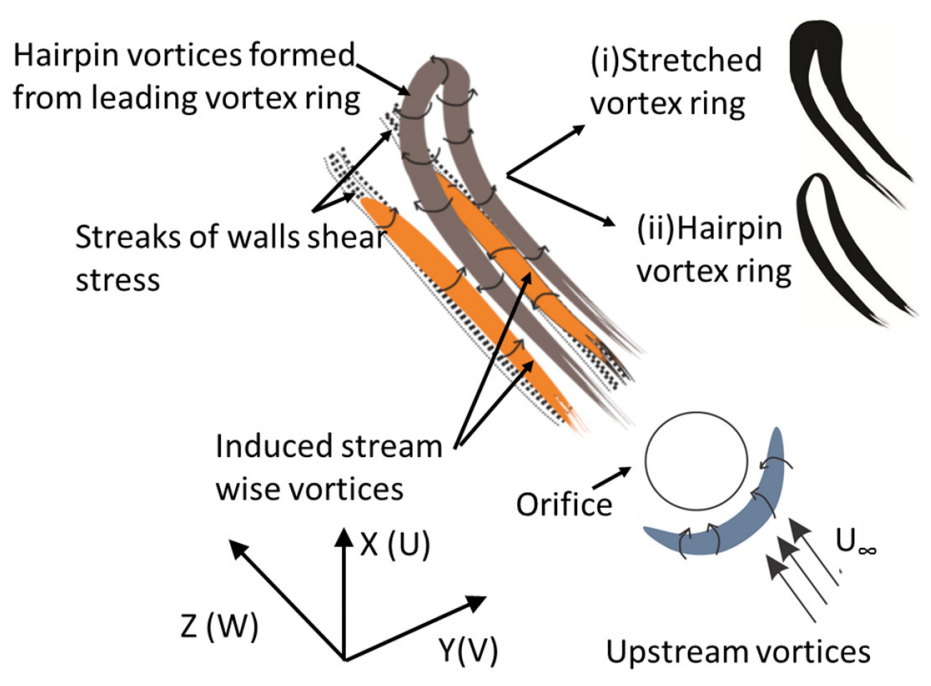

(a) Hairpin and stretched vortices

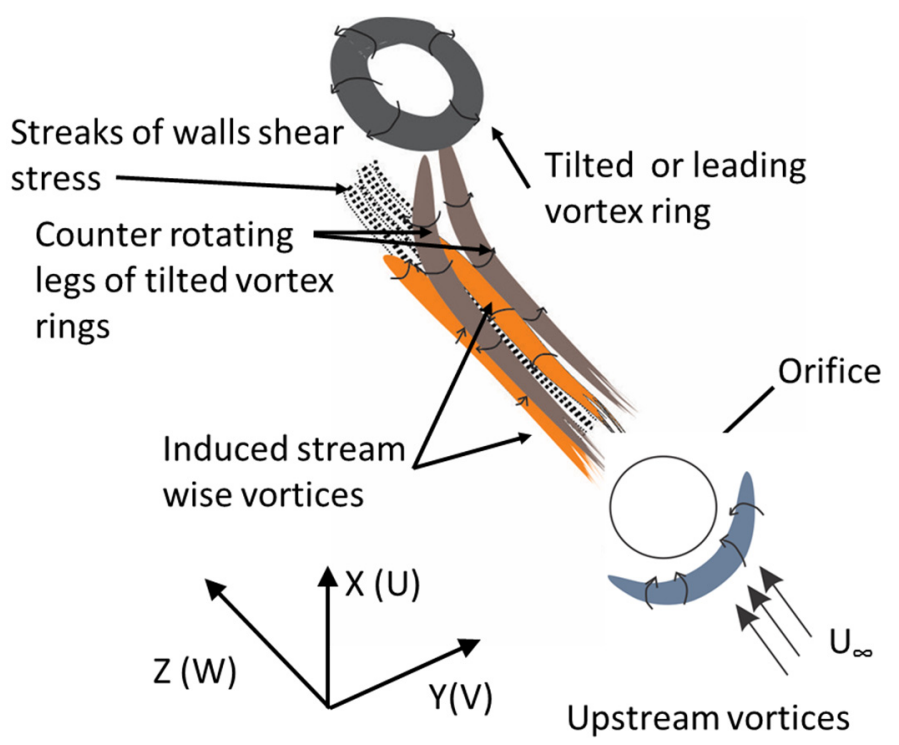

(b) Tilted and distorted vortex ring

Figure 6.9: Schematic representing the synthetic jet in crossflow having (a) hairpin and stretched vortices and (b) tilted and distorted vortex ring with stretched traling jet.

The tilting or stretching of synthetic jet in crossflow is a key feature in the formation of hairpin vortices aligned with crossflow direction. Within the boundary layer, the vortex tilting or stretching occurs because of vorticity variations of vortex ring at orifice exit. The sectional view of the vortex rings gives two counter-rotating (CR) vortices separated by a distance equal to the diameter of the ring. Due to crossflow, the vorticity strength of upstream counter-rotating vortices of synthetic jet is adversely affected by both (i) the 
upstream vortices also called horse-shoe vortices generated due to blockage of the incoming crossflow boundary layer by the jet and (ii) the shear layer of incoming flow. The regions which affect the upstream $\mathrm{CR}$ vortices are indicated as ' 1 ' and '2' in Figure 6.10. In region ' 1 ', the rotation of CR vortices is opposite to that of the horse-shoe vortices while in region ' 2 ', the top part of CR vortex is opposed by crossflow velocity as shown in Figure 6.10. On other hand, the downstream CR vortices is favoured by crossflow at the top (region 3) because both have identical sense of flow motion. Especially at higher VR, the blockage of crossflow by the jet creates a wake region (region 4) in the downstream side of synthetic jet and may lead to enhancement of the strength of downstream CR vortices. When the suction stroke begins, the large amount of fluid is sucked inside the cavity from upstream side because of the lower velocity of the crossflow caused by the blockage of the jet in this region. From the above discussion, it is quite clear that combined effect of shear layer interaction in the four region indicated earlier produces an asymmetric vortex ring with varying vorticity content around the circumference of the ring. The upstream part of the ring has minimum vorticity while the vorticity content increases slowly towards the downstream along the circumference. Consequently, this asymmetric distribution of vorticity results in a doughnut shape ring having non-uniform cross-sectional area, being minimum at upstream while maximum at downstream. Since the variation in vorticity causes a variation in self-induced velocity, the differential lift is experienced by the vortex ring. The downstream side of vortex ring experiences higher lift in comparison to the upstream side. In case of hairpin vortices, the difference of self-induced velocity is such that the downstream part of leading vortex ring lifts up while the upstream part remains attached to the wall and interact with the boundary layer. The velocity variation within the boundary layer further provides a differential shearing force on the asymmetric vortex ring causing a huge stretching in streamwise direction forming the legs of hairpin vortices. 


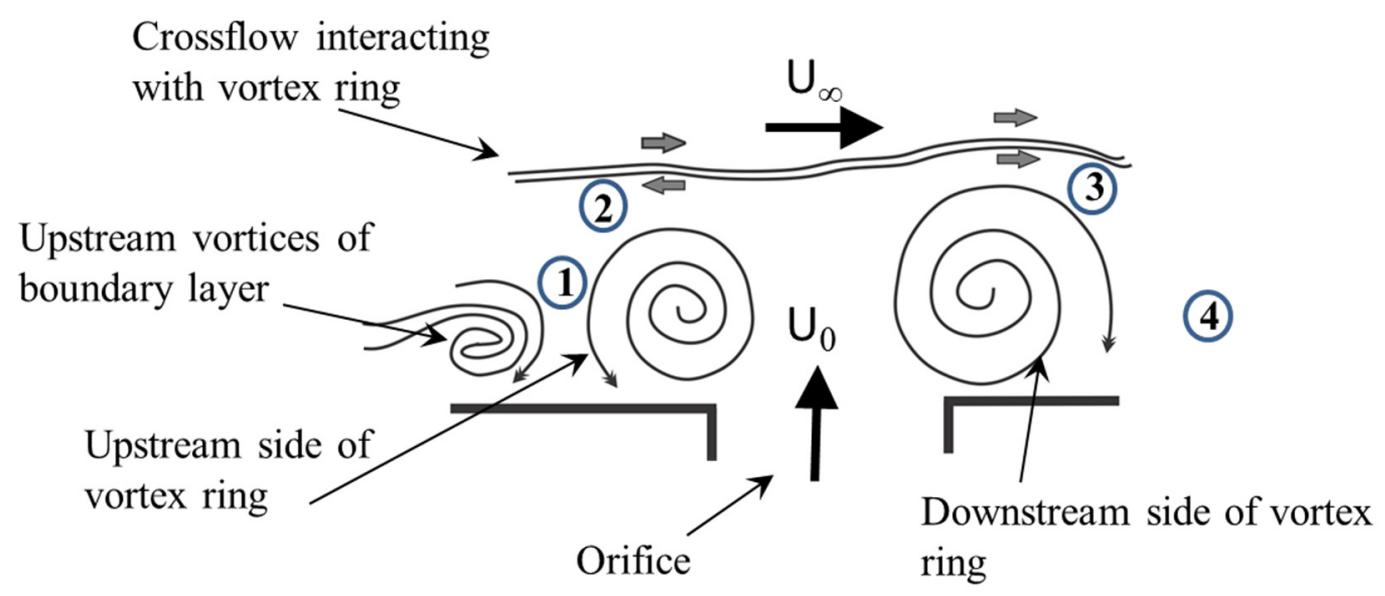

Figure 6.10: Evolution of synthetic jet in crossflow

\subsubsection{Time averaged flow field}



(a)

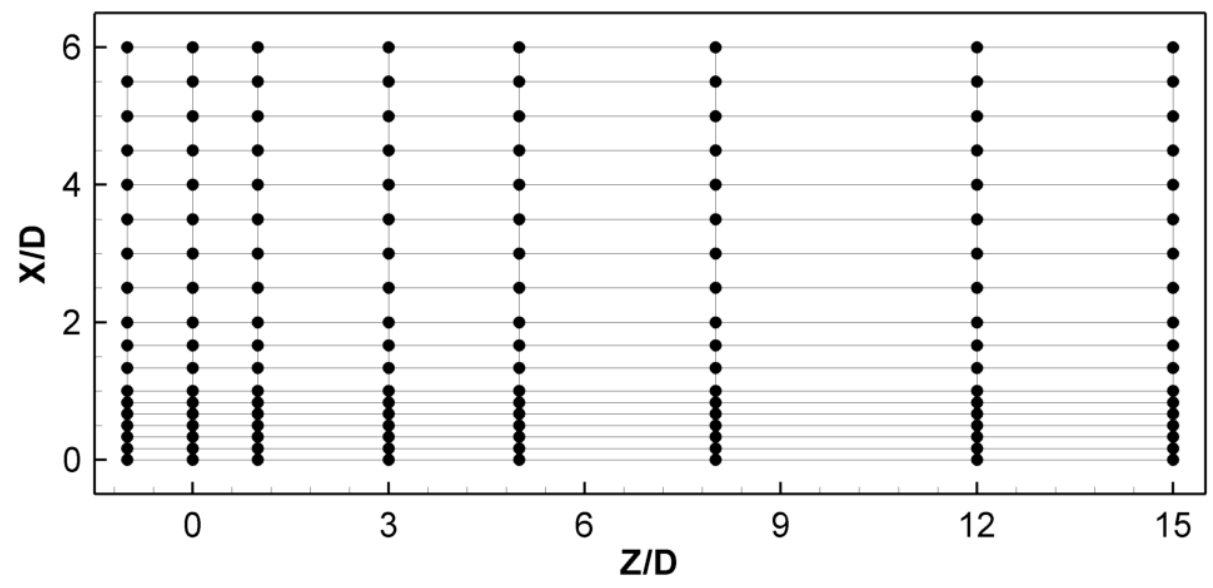

(b)

Figure 6.11: (a) Grid points for LDV measurement for comparing the torpedo shape model with synthetic jet orifice at $0 \mathrm{~Hz}$ actuation frequency and without the presence of crossflow and (b) grid points for velocity measurement in crossflow for the conditions shown in Table 6.2. 
The results of flow visualization study mainly present the instantaneous flow field of synthetic jet in crossflow. The time-averaged field provides the large scale coherent flow structures which are generally boundary dependent. The crossflow component of velocity (W) and the vertical velocity component along jet axis (U) are averaged over 60 cycles of synthetic jet actuation. The time-averaged flow field on a plane passing through the orifice centre is measured for two conditions: (i) case with orifice open without any actuation and (ii) the case having no orifice in place. The matrix for measurement points are shown in Figure 6.11 (a). Near the wall the spacing are $0.2 \mathrm{~mm}$ up to $\mathrm{X} / \mathrm{D}=0.75$ which is followed by a spacing of $0.5 \mathrm{~mm}$ thereafter. The grid of measurement points in XZ-plane for $\mathrm{Y}=0$ and in XY-plane for $\mathrm{Z} / \mathrm{D}=1,5$ and 12 for synthetic jet in crossflow are shown in Figure $6.11(b)$.

\subsubsection{Velocity flow field with and without orifice on torpedo shape model}
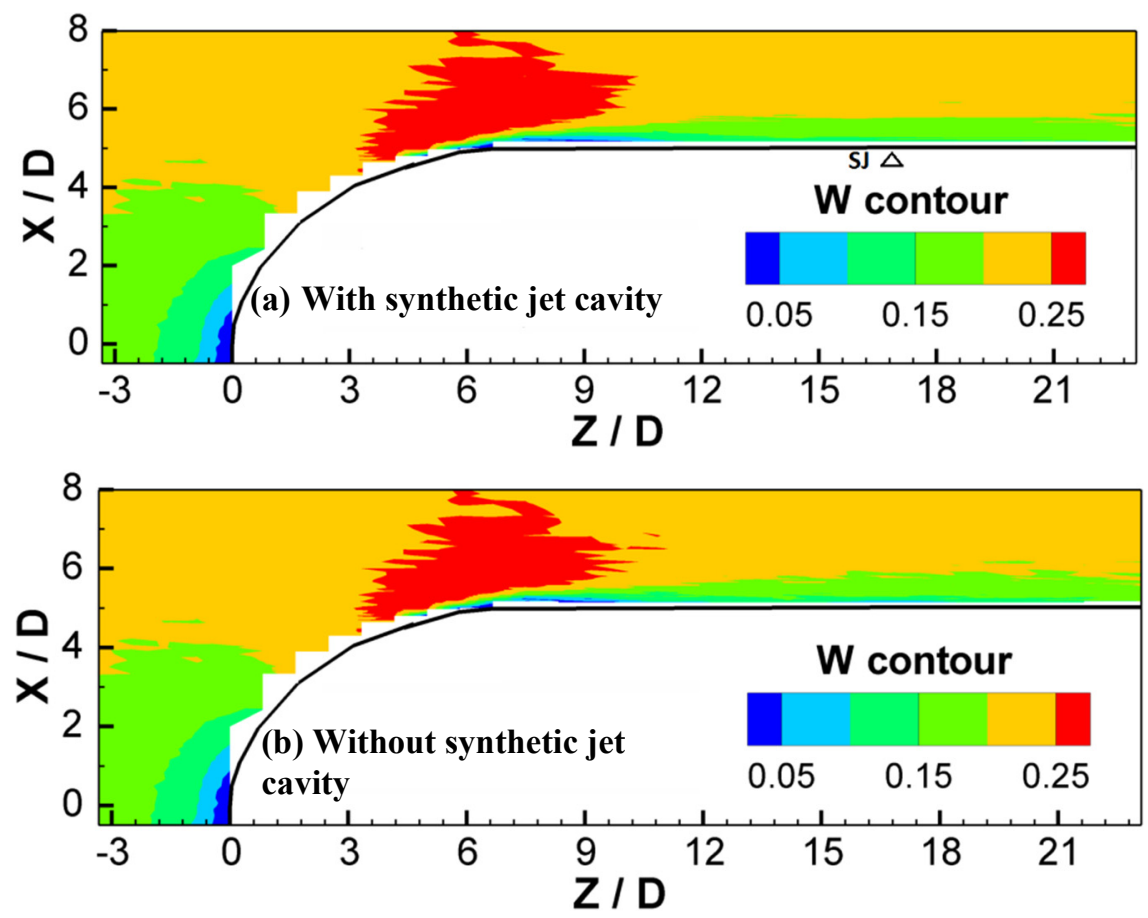

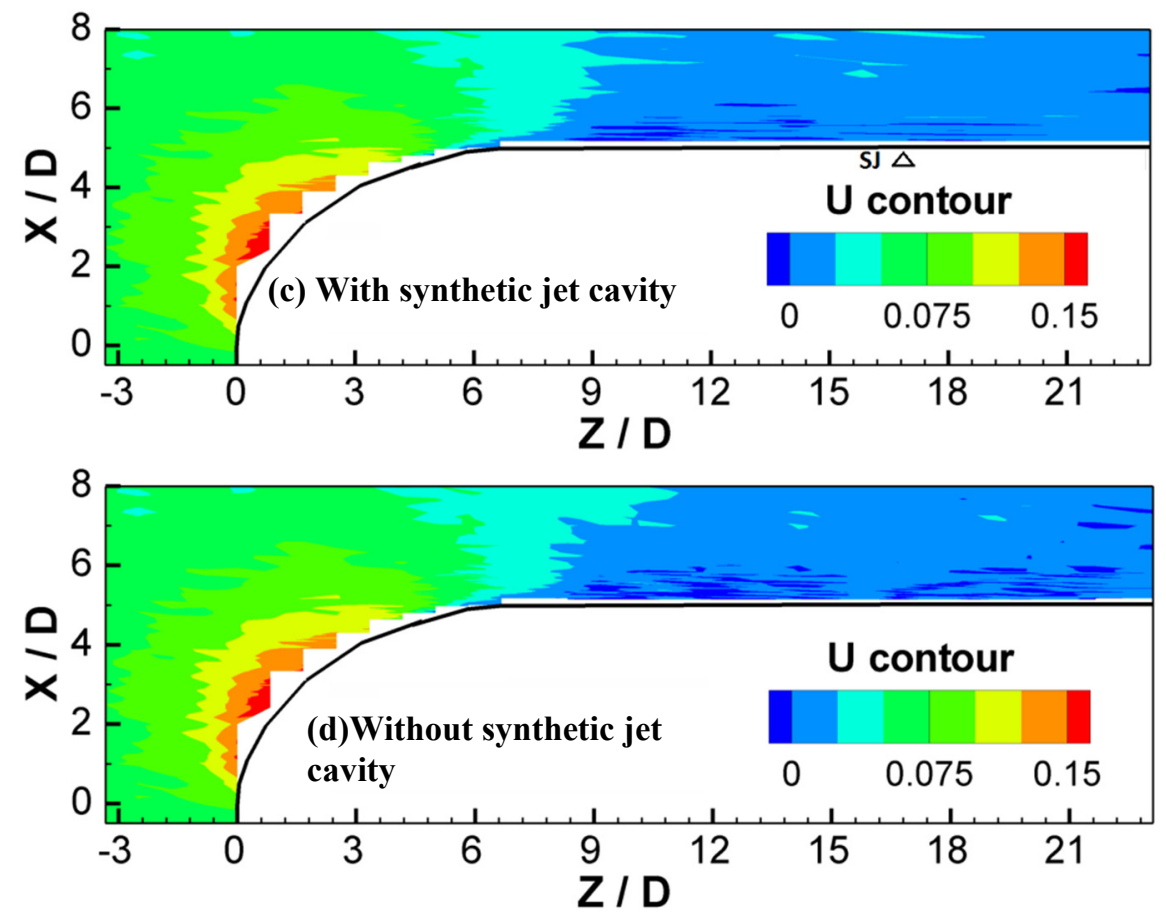

Figure 6.12: Velocity contours over the torpedo shape model with and without the cavity of synthetic jet (SJ) in test section of water tunnel at $0.2 \mathrm{~m} / \mathrm{s}$. (a) and (b) are the velocity component in crossflow direction and (c) and (d) are velocity component in synthetic jet direction.

The time-averaged flow field on the above mentioned plane for torpedo shaped model placed inside the test section of water tunnel has been measured using LDV. The measurements are taken for all three values of crossflow. The position of the orifice is shown using an upward triangle located between $\mathrm{Z} / \mathrm{D}=15$ and 18 . The model is positioned $170 \mathrm{~mm}$ away from two transverse sidewalls, $120 \mathrm{~mm}$ from the bottom wall, and $120 \mathrm{~mm}$ from the free surface of the water in the test section. The contours of both the crossflow and vertical velocity have been depicted in Figure 6.12 for crossflow velocity of $0.2 \mathrm{~m} / \mathrm{s}$. No significant variation in the velocity field has been observed for the two cases with and without orifice in place. As expected, the flow is found to be almost zero at the front stagnation region while the blockage created by the presence of the model accelerates the flow in the region between the sidewall of the test section and side straight wall of the model. The almost zero or negligible vertical velocity $(\mathrm{U})$ in the gap region beyond $\mathrm{Z} / \mathrm{W}=$ 9 ensures a parallel flow around the model. Moreover, the velocity measurements at various 
crossflow velocities provide us with the estimation of reference boundary thickness discussed earlier. Also the velocity field reveals the insignificant blockage created by the presence of model in the tunnel.

\subsubsection{Effect of actuation frequency and velocity ratio}

The vertical variation of velocity components at eight different locations namely, $\mathrm{Z} / \mathrm{D}=$ $1,0,1,3,5,8$ and 12 on streamwise plane (XZ) passing through the center of the orifice is shown in Figure 6.13-6.18. The actuation frequency has been varied in the range of $0-6$ $\mathrm{Hz}(0,2,4$ and $6 \mathrm{~Hz})$ for three different crossflow velocities $\left(\mathrm{U}_{\infty}=0.072,0.2\right.$ and $\left.0.32 \mathrm{~m} / \mathrm{s}\right)$. The velocity variation with no jet indicated using solid symbols and dashed line is taken as reference for comparing the velocity variation for the various frequencies.

For a crossflow velocity of $U_{\infty}=0.072 \mathrm{~m} / \mathrm{s}$, the vertical variations of crossflow velocity component for various frequencies at different axial locations are depicted in Figure 6.13 . The velocity variations at an upstream location of the orifice, $Z / D=-1$ reveal almost similar distribution near the wall except for frequencies of 4 and $6 \mathrm{~Hz}$ where velocity with positive velocity has been observed. The distribution of vertical velocity reveals the evidence of the negative value shown in Figure 6.14 at the same location. As discussed earlier the synthetic jet draws more fluid from the upstream side of orifice at higher frequencies thus increasing the crossflow velocity within the boundary layer along with a downward vertical velocity (negative velocity). The streamwise velocity component (W) decreases near the orifice exit $(\mathrm{Z} / \mathrm{D}=0)$ due to combined effect of blockage of crossflow during ejection stoke and suction of boundary layer fluid during suction stroke. At locations downstream of the orifice, the crossflow velocity near the wall is found to show velocity deficit in comparison to the reference velocity variation and two localised peaks indicating the two trajectories of vortices namely leading and trailing ones (see Figure 6.6 (a) and Figure 6.7 (a)). With increasing downstream distance the deficit decreases indicating 
weakening of the vortices in the two rows probably because of vortex diffusion and entrainment of outer fluid into the jet region. The variation of vertical velocity component (U) (Figure 6.14) at locations downstream of orifice shows positive velocity for two higher frequencies (4 and $6 \mathrm{~Hz}$ ) indicating the upward motion of the jet, the magnitude of which decreases with increasing downstream distance. Also increasing width of the velocity surplus in vertical velocity component indicates the spreading nature of the jet with increasing axial distance. The probable reason of this behaviour may be the following. The train of vortices generated in synthetic jet produces a shielding effect to the crossflow. This shielding generates a low-pressure region behind the synthetic jet in downstream and results in decreases of the velocity. This wake region draws more fluid from the adjoining region and increases the spreading. From the velocity variation, the blockage effect on streamwise velocity is found to be limited to $\mathrm{Z} / \mathrm{D}=5$ beyond which no substantial velocity deficit is observed. Similar observation has also been reported by Lardeau and Leschziner (2011) in their numerical study of circular synthetic jet in crossflow.

At higher crossflow velocity $\left(\mathrm{U}_{\infty}=0.2\right.$ and $\left.0.32 \mathrm{~m} / \mathrm{s}\right)$, the vertical variation of the two components of velocity does not reveal significant changes in comparison to the baseline case. It is obvious that the higher crossflow velocity corresponds to lower velocity ratio. This in turn diminishes the relative strength of the jet experiencing an increasing higher crossflow velocity. Figure 6.15 confirms this through a small velocity deficit near the wall at locations close to the orifice. Similar observation has also been observed in case of vertical velocity (Figure 6.16) where changes have been observed only at the highest frequency. The velocity variations for $\mathrm{U}_{\infty}=0.32 \mathrm{~m} / \mathrm{s}$ at various frequencies (see Figure 6.17 and Figure 6.18) reveal even lower influence of the jet on the crossflow velocity except close to the wall. This is because of the fact that at higher crossflow velocity, the jet 
structures confine themselves near the wall unlike at higher velocity ratio where the crossflow gets affected significantly over the entire vertical span of the flow domain.
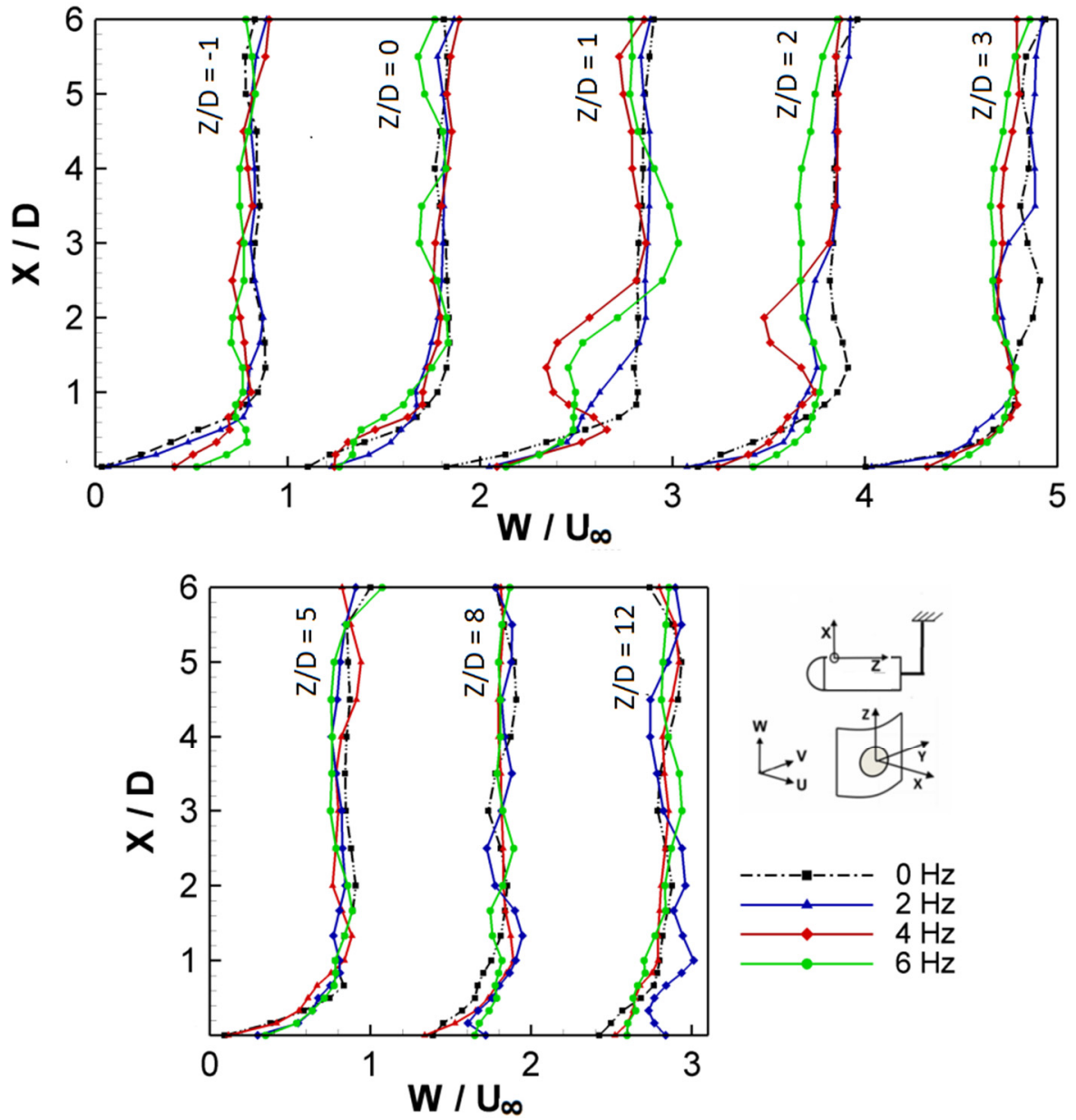

Figure 6.13: Time averaged velocity $(W)$ parallel to crossflow at eight different locations $Z / D=$ $1,0,1,2,3,5,8$ and 12 for $0,2,4$ and $6 \mathrm{~Hz}$ actuation frequency at $U_{\infty}=0.072 \mathrm{~m} / \mathrm{s}$. ( ${ }^{*}$ For clarity the velocity lines are shfted on $\mathrm{X}$-axis by a step size equal to one).



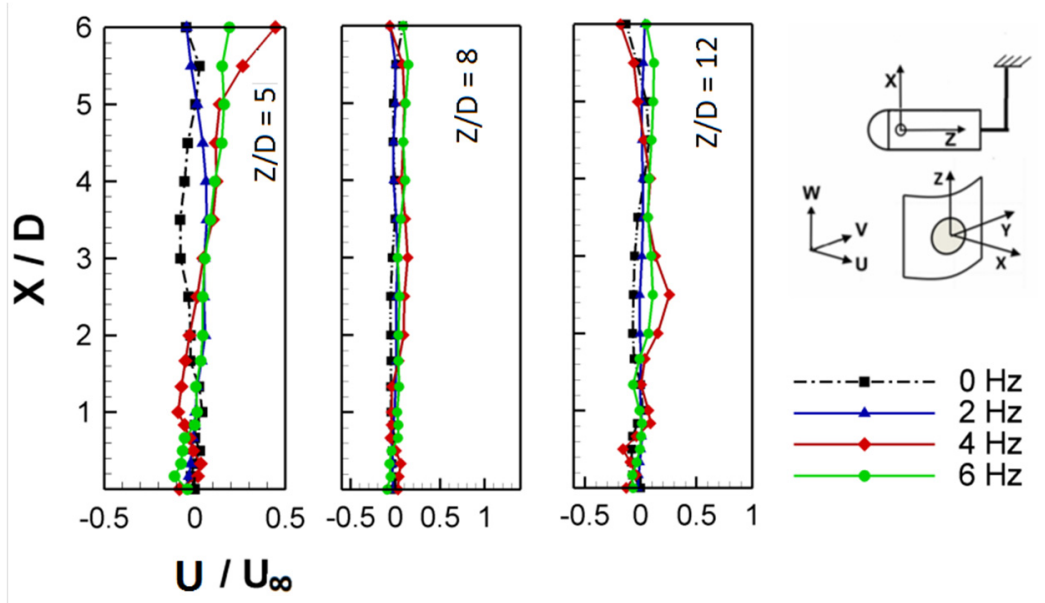

Figure 6.14: . Time averaged velocity $(U)$ parallel to crossflow direction at eight different locations $\mathrm{Z} / \mathrm{D}=-1,0,1,2,3,5,8$ and 12 at $0,2,4$ and $6 \mathrm{~Hz}$ actuation frequency at $\mathrm{U}_{\infty}=0.072 \mathrm{~m} / \mathrm{s}$.
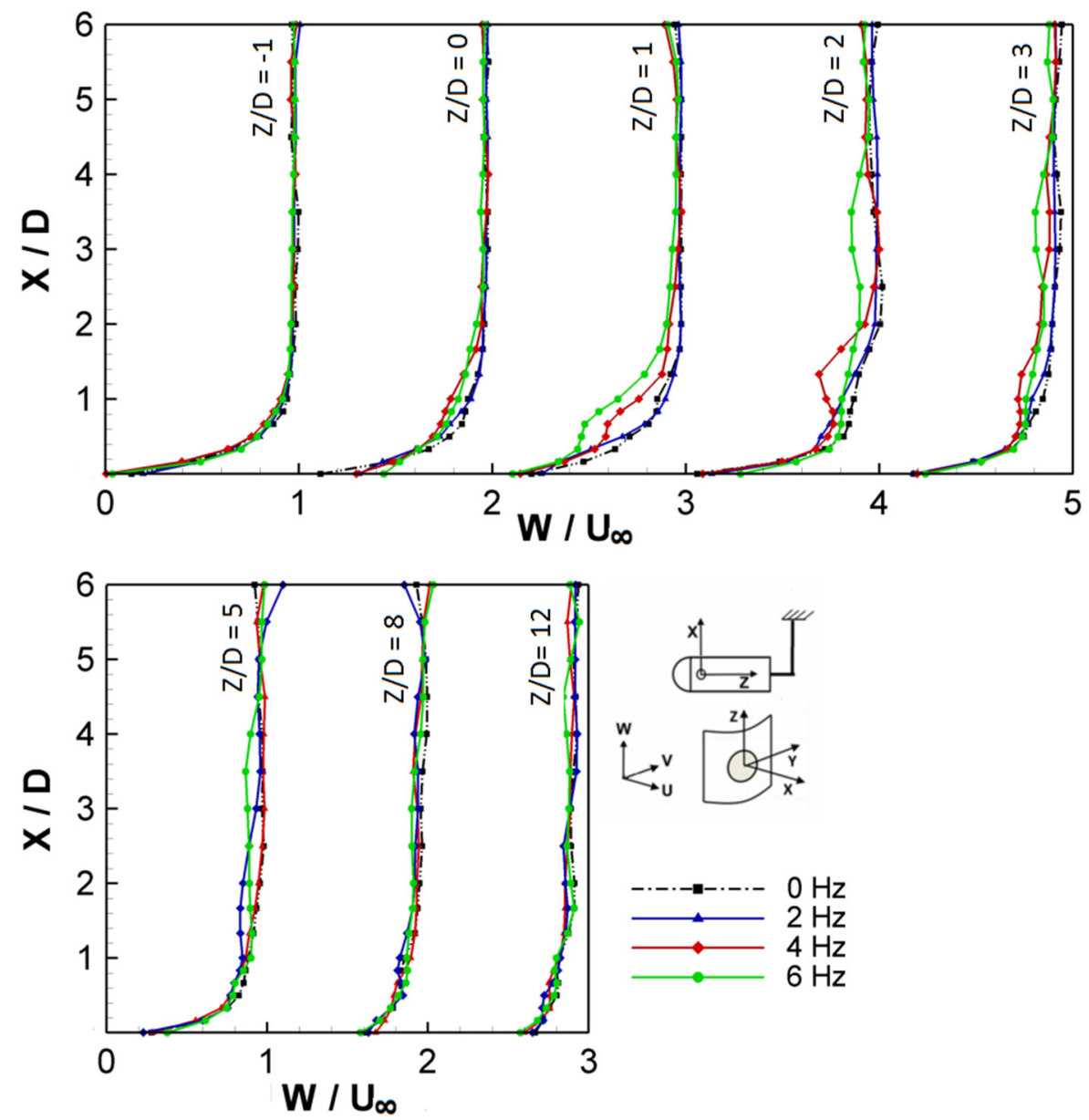

Figure 6.15: Time averaged velocity $(W)$ parallel to crossflow direction at eight different locations $\mathrm{Z} / \mathrm{D}=-1,0,1,2,3,5,8$ and 12 for $0,2,4$ and $6 \mathrm{~Hz}$ actuation frequency at $\mathrm{U}_{\infty}=0.2 \mathrm{~m} / \mathrm{s}$. (*For clarity the velocity lines are shfted on $\mathrm{X}$-axis by at a step size equal to one). 

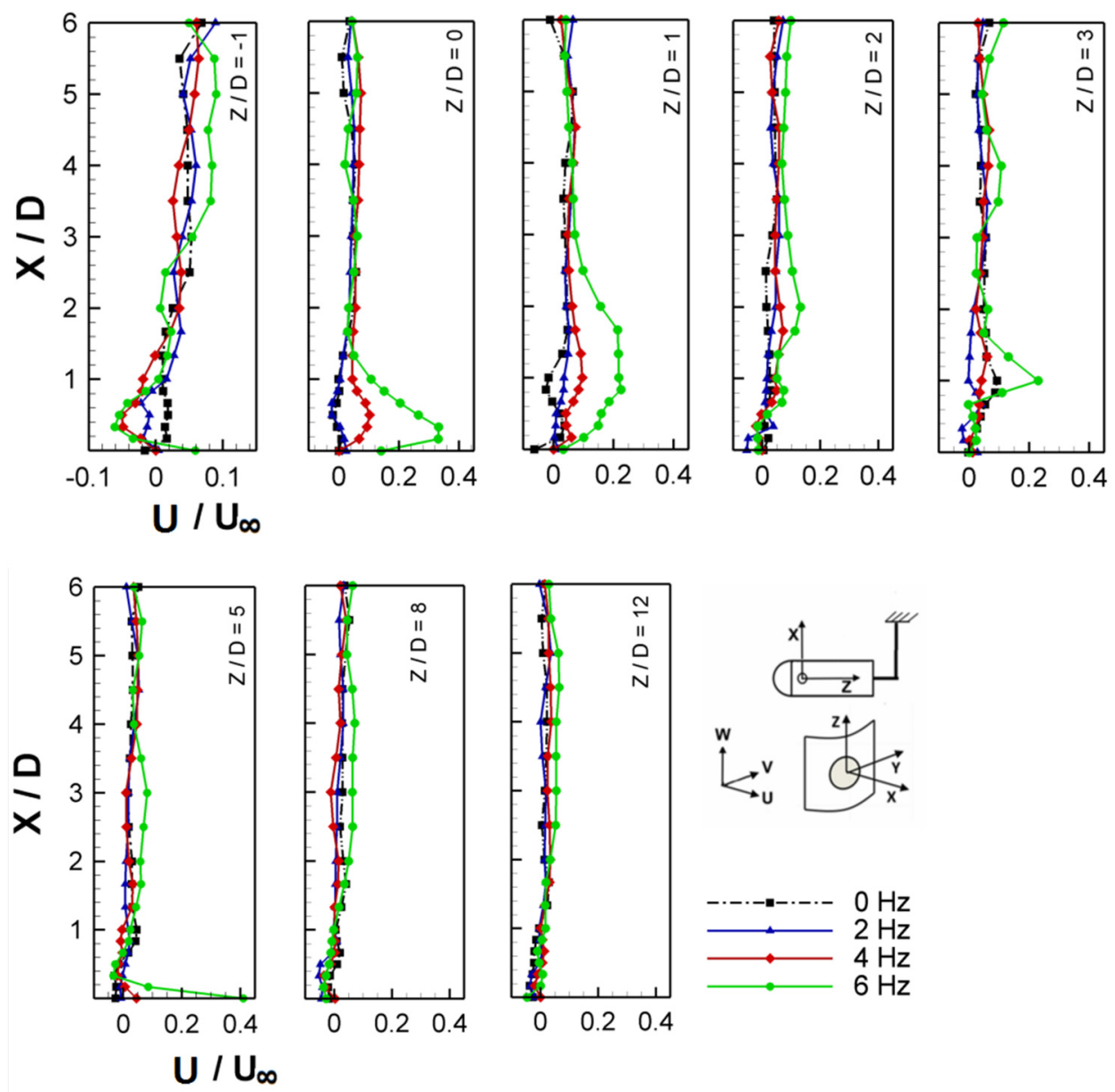

Figure 6.16: . Time averaged velocity (U) parallel to crossflow direction at eight different locations $\mathrm{Z} / \mathrm{D}=-1,0,1,2,3,5,8$ and 12 at $0,2,4$ and $6 \mathrm{~Hz}$ actuation frequency at $U_{\infty}=0.2 \mathrm{~m} / \mathrm{s}$.

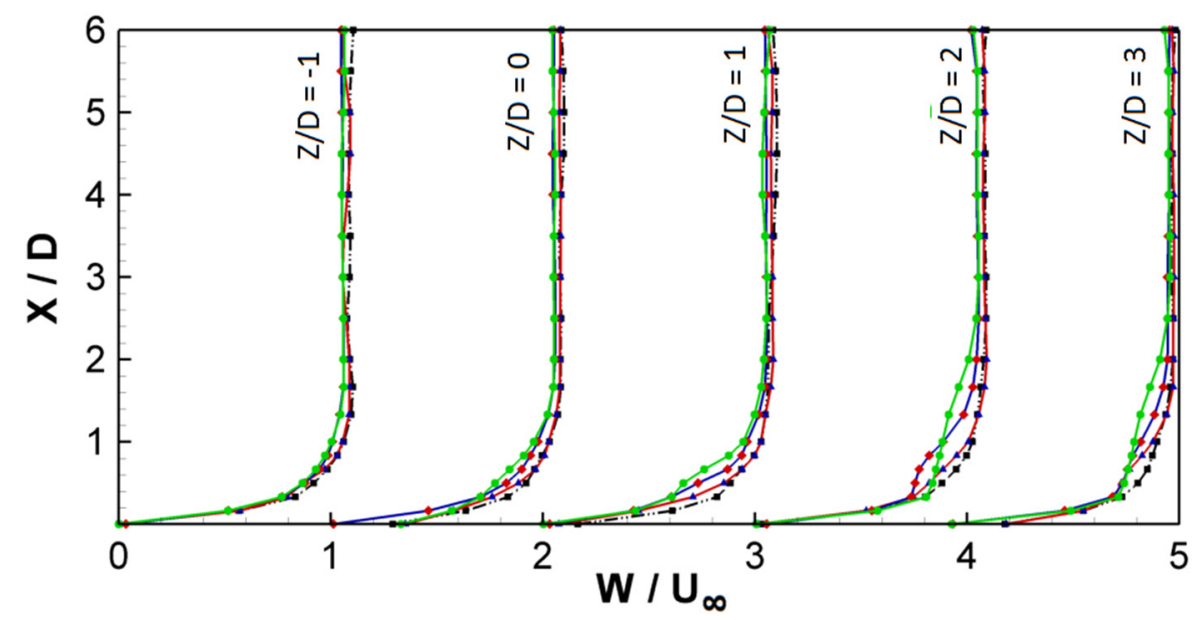




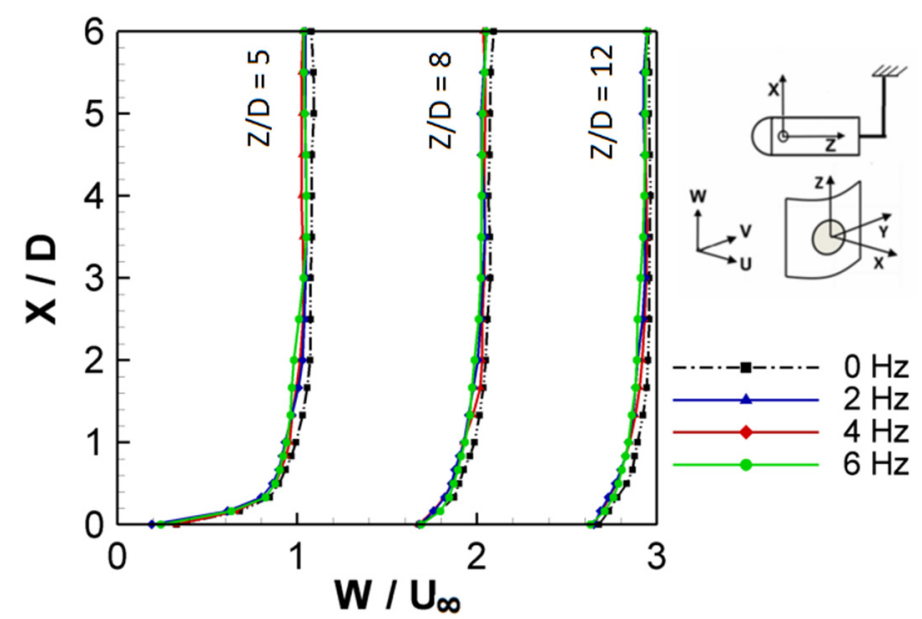

Figure 6.17: Time averaged velocity component $(W)$ parallel to crossflow direction at eight different locations $Z / D=-1,0,1,2,3,5,8$ and 12 at $0,2,4$ and $6 \mathrm{~Hz}$ actauation frequency at $\mathrm{U}_{\infty}=$ $0.32 \mathrm{~m} / \mathrm{s}$. (For clarity the velocity lines are shfted on $\mathrm{X}$-axis by at a step size equal to one)


Figure 6.18: Time averaged velocity component(U) parallel to cross-flow direction at eight different locations $\mathrm{Z} / \mathrm{D}=-1,0,1,2,3,5,8$ and 12 at $0,2,4$ and $6 \mathrm{~Hz}$ actuation frequency at $\mathrm{U}_{\infty}=$ $0.32 \mathrm{~m} / \mathrm{s}$. 


\subsection{Closure}

In the presence of crossflow, the behaviour of synthetic jet changes significantly particularly at higher velocity ratio as the formation, convection, and interaction among vortices are influenced by the crossflow. Analysis of the flow visualization images reveal two major types of vortex rings: (a) stretched vortex ring and (b) tilted and distorted vortex ring. The tilting or stretching of synthetic jet in crossflow is a key feature in the formation of hairpin vortices aligned with crossflow when residing within the boundary layer.

Comparison of time-averaged velocity variation in the vertical direction reveals the formation of two local peaks at higher velocity ratio and higher frequency of actuations. The crossflow is also found to be significantly affected by the high velocity ratio jet. On the other hand, the low velocity ratio jet in crossflow does not show substantial influence on the crossflow except near the wall. The high velocity ratio jet shows the formation of wake behind the jet region which is believed to be generated because of the blockage of the crossflow created by the jet. 


\section{Chapter 7}

\section{Conclusions and scope for future work}

\subsection{Conclusions}

The present dissertation reports the characteristics of circular and non-circular synthetic jets in quiescent and crossflow condition using water as the working fluid. The objectives of the present study are three fold. First objective is to design and fabricate a water tank fitted with synthetic jet generator on one of its sidewall for study in quiescent flow condition and a study of torpedo shaped model with built-in synthetic jet. The second objective is the characterization of the circular and rectangular (different aspect ratios) shaped synthetic jet in quiescent flow at different actuation frequencies and amplitudes using LIF and hot-film anemometry. The third objective is to study the circular synthetic jet in torpedo shaped model at various crossflow velocities and actuation frequencies. The LIF, bulk-flow visualization, hot-film and LDV measurements are carried out to achieve these objectives. The major conclusions drawn from the present work are summarized as follows:

1. Circular orifice: The sequence of LIF images reveal that after the vortex overshoot region $(X / D<3)$, the spreading of circular synthetic jet is the highest for $8 \mathrm{~mm}$ diameter orifice $(\mathrm{Re}=506-9533, \mathrm{St}=0.14-0.05)$ while it is the lowest for $10 \mathrm{~mm}$ diameter orifice $(\mathrm{Re}=462-7380, \mathrm{St}=0.28-0.10)$. However, for $13 \mathrm{~mm}$ diameter orifice $(\mathrm{Re}=$ $370-5906, \mathrm{St}=0.6-0.22)$ no spreading is found up to $\mathrm{X} / \mathrm{D}=8$. Four distinct forms of 
synthetic jet are observed from the parametric studies (indicated in the bracket) carried out using circular orifices:

- Partially formed synthetic jet $\left(\mathrm{L} / \mathrm{D}_{\mathrm{h}}=1.6, \mathrm{Re}=370\right)$

- Synthetic jet without trailing jet $\left(\mathrm{L} / \mathrm{D}_{\mathrm{h}}=3.6-7.1, \mathrm{Re}=462-1062\right)$

- Synthetic jet with coherent trailing jet $\left(\mathrm{L} / \mathrm{D}_{\mathrm{h}}=7.2-10, \mathrm{Re}=1462-7380\right)$

- Synthetic jet with chaotic or turbulent trailing jet $\left(L / D_{h}=14-20, \operatorname{Re}=6933-9533\right)$.

2. Rectangular orifice: The presence of corners in rectangular orifices significantly alters the vortex formation mechanism and its propagation. At low Reynolds number and formation number, the velocity of newly formed vortex rings is observed to be small and vortex shape is extensively governed by the suction period of the following cycle causing a large variation in the flow pattern. At certain distance downstream from the orifice exit, the flow pattern changes from laminar to chaotic or probably turbulent more rapidly than the circular orifices operating at same actuation frequency. Different patterns of flow structures observed in rectangular orifices $\left(D_{h}=12\right)$ are summarized below:

- The vortex ring experiences axial switching for AR2 (aspect ratio of 2) at $\mathrm{L} / \mathrm{D}_{\mathrm{h}}=$ $2.9-4.0$ and $\operatorname{Re}=1549-3435$.

- Bifurcation of the vortex ring is observed during the first axial switching for AR2 at $\mathrm{L} / \mathrm{D}_{\mathrm{h}}=1.5-2.1$ and $\mathrm{Re}=215-491$.

- For AR4, partial suction of ejected vortex ring is noticed for $\mathrm{L} / \mathrm{D}_{\mathrm{h}}=1.1$ and $\mathrm{Re}=$ 155.

- At AR4, breaking of vortex ring into multiple tiny rings at close to the orifice exit is observed for $\mathrm{L} / \mathrm{D}_{\mathrm{h}}=1.5-2.9$ and at relatively higher Reynolds number, $\mathrm{Re}=354$ - 2473. The jet with higher $\mathrm{L} / \mathrm{Dh}_{\mathrm{h}}$ and Reynolds number also reveals a large spreading and mixing in the far field region. 
- Vortex rings experience a maximum of three axial-switching before their breakup. However, the third axial switching is found to be weak and difficult to detect.

3. Time-Averaged Flow Field for Circular and Rectangular orifice: Based on the variation of time-averaged velocity distribution (streamwise velocity) along the synthetic jet centerline for both circular and non-circular orifice the synthetic jet evolution can be broadly classified into four zones:

- Vortex rollup and suction region $\left(\mathrm{X} / \mathrm{D}_{\mathrm{h}} \leq 3\right)$.

- Steadily translating vortex ring region $\left(\mathrm{X} / \mathrm{D}_{\mathrm{h}}=3-8\right)$.

- Vortex breakdown region $\left(\mathrm{X} / \mathrm{Dh}_{\mathrm{h}}=4-12\right)$.

- Dissipation of small-scale vortices $\left(\mathrm{X} / \mathrm{D}_{\mathrm{h}}=8-15\right)$.

Close observation of the sequence of LIF images shows that after a certain downstream distance, the trajectories of the vortex rings are not unique and drifts away from the synthetic jet centerline in all directions indicating possible blooming of jet.

4. Spectral analysis: Behavior of both circular (10 $\mathrm{mm}$ diameter) and rectangular orifices (aspect ratio 1,2 and 4) actuated at 1,6 and $18 \mathrm{~Hz}(\mathrm{Re}=155$ to 20374 and $\mathrm{St}=0.1$ to 68) have been discussed using velocity-time trace, power spectral density distribution and Choi-William wavelet transformation. The major findings from these analysis are mentioned below.

- In time domain representation of velocity signal, the coherent structures (vortex rings in the present study) represent themselves in the form of peaks evenly distributed on time axis, the number of which depends on actuation frequency. On the other hand, these peaks in frequency domain (power spectral density distribution) are observed at actuation frequency and its harmonics. These peaks in time and frequency domains are clearly observed up to a large downstream location for circular orifice compared to that of rectangular orifices. 
- In joint time-frequency representation (wavelet transformation), the total energy of the velocity signal is primarily distributed in the form of ribs and bands. Ribs parallel to the frequency axis show the energy distribution of the vortex ring in frequency domain and bands parallel to the time axis provides evidence of periodicity or pulsating nature of coherent structures at different harmonics of the actuation frequency. Synthetic jets having trailing jet are represented by bands parallel to time axis at different harmonics. For chaotic/turbulent jet, first harmonic shares maximum amount of energy unlike laminar vortex rings where the energy is distributed at different harmonics.

5. Bifurcation: For rectangular synthetic jet $\left(A R=2, D_{h}=9.33 \mathrm{~mm}\right)$ bifurcation has been observed at actuation frequencies of 1, 2 and $3 \mathrm{~Hz}$. Following observations are made based on the detailed study of bifurcation and axial switching of leading vortex.

- Vortex splitting or bifurcation is found on plane coinciding with the minor axis of the orifice. The divergence angles (using LIF images) obtained between the two trajectories of vortex rings are $124^{\circ}(\mathrm{Re}=275, \mathrm{St}=0.33)$ at $1 \mathrm{~Hz}, 112^{\circ}(\mathrm{Re}=1101$, $\mathrm{St}=0.13)$ at $2 \mathrm{~Hz}$ and $68^{\circ}(\mathrm{Re}=1309, \mathrm{St}=0.17)$ at $3 \mathrm{~Hz}$ of actuation frequency.

- Axial switching of vortex ring has been observed at 1,2 and $3 \mathrm{~Hz}$ of actuation frequency for $\mathrm{Re}=625,1348$ and 2038 and $\mathrm{St}=0.15,0.11$ and 0.11 respectively.

- The main source of bifurcation is believed to be the variation in vortex tube cross section that results in varying self-induced velocity along the length of the vortex tube.

6. Synthetic jet in crossflow: In the presence of crossflow, the behavior of synthetic jet changes significantly because the crossflow modifies the formation and advection behavior of the jet. The comparative study of synthetic jet $(\mathrm{D}=6 \mathrm{~mm} \mathrm{~L} / \mathrm{D}=6.08)$ has been carried out using various crossflow velocities $\left(\mathrm{U}_{\infty}=0.072,0.2\right.$ and $\left.0.32 \mathrm{~m} / \mathrm{s}\right)$ and 
different actuation frequencies $(2,4$ and $6 \mathrm{~Hz})$ with synthetic jet at various Reynolds numbers (Ress $=732,1372$ and 2058 respectively in quiescent flow condition). It has been observed that the flow structures primarily depend on the velocity ratio, which is a function of actuation frequency and crossflow velocity.

- For the velocity ratio in the range of $0.61-1.14(\operatorname{ReSJ}=732-1372)$, the leading vortex is followed by strong trailing jet. The asymmetric roll up of synthetic jet at orifice exit is strongly influenced by the momentum of crossflow.

- The stretched vortex rings with lifted downstream head with the legs within the reference boundary layer are observed for the velocity ratio between 1.69-1.72 $($ ResJ $=732-1372)$.

- For velocity ratio equal to 3.18 and $4.77($ Ress $=1372-1372)$, because of the changes in the self-induced velocity of the leading and trailing (leg) vortices, two different trajectories of vortices are observed one for the leading ones and the other for the trailing ones.

- Time-averaged velocity reveals that at low crossflow velocity and higher actuation frequency, synthetic jet draws more fluid from the upstream side of the orifice that increases the velocity within the boundary layer in upstream side of the orifice. The streamwise velocity decreases near the orifice exit due to the blockage provided by the jet and suction of the boundary layer fluid into the cavity during the suction period. At higher velocity ratio, the bulk of crossflow is influenced by the jet while at lower velocity ratio; flow near the wall is seen to be affected. 


\subsection{Future work}

Future research work on this topic should include the following.

The circular and square synthetic jets have larger penetration depth but lower spreading in transverse direction whereas the rectangular synthetic jet shows less penetration but higher spreading in transverse direction. Apart from circular and rectangular orifices, the synthetic jet with orifices having other shapes such as elliptical, triangular, or irregular shape needs to be investigated for a wide range of parameters.

Since, the bifurcating synthetic jet demonstrated a good mixing quality, elaborate experiments can shed more light on the physics responsible for bifurcation process. Judicious choice of parameters and instantaneous quantitative measurements using particle image velocimetry (PIV) should help in unearthing the physical mechanism of bifurcation of jet.

The present study using single jet can also be extended for near wall flow control around the torpedo shaped body using multiple jets. In addition, the force measurements should also be carried out for such applications.

The present study has been carried out with normal orientation of the synthetic jet with respect to crossflow. Additional research can be carried out for parametric study of synthetic jet with different shapes, size and at various inclination angles to the crossflow. 


\section{References}

[1] Akhmetov, D. G. \& Kisarov, O. P., 1966. Hydrodynamic structure of a vortex ring. Journal of Applied Mechanics and Technical Physics, 7(4), pp. 120-123.

[2] Amitay, M. \& Cannelle, F., 2006. Evolution of finite span synthetic jets. Physics of Fluids, 18(54101).

[3] Amitay, M. \& Cannelle, F., 2007. Transitory behavior of synthetic jets. Physics of Fluids, Volume 19, pp. 1 - 15.

[4] Amitay, M. \& Glazer, A., 2002. Controlled transients of flow reattachment over stalled airfoils. International Journal of Heat and Fluid Flow, Volume 23, pp. 690699.

[5] Amitay, M., Honohan, A., Trautman, M. \& Glezer, A., 1997. Modification of the Aerodynamics Characteristics of Bluff Bodies Using Fluidic Actuators. AIAA Paper 97-2004.

[6] Amitay, M. \& Pitt, D. a. G. A., 2002. Separation control in duct flows. Journal of Aircraft, 39(4), pp. 616-620.

[7] Amitay, M. et al., 2001. Modification of the Aerodynamics Characteristics of an Unconventional Airfoil Using Synthetic Jet Actuators. AIAA Journal, 39(3), pp. 361-370.

[8] Amitay, M. et al., 2001. Aerodynamic flow control over an unconventional airfoil using synthetic jet actuators. AIAA Journal, 39(3), pp. 361-370.

[9] Anderson, E. \& Grosenbaugh, M., 2005. Jet flow in steadily swimming adult squid. The Journal of Experimental Biology, Volume 208, pp. 1125-1146.

[10] Anna., P. \& Michael Amitay, M., 2006. Electronic Cooling Using Synthetic Jet Impingement. Journal of Heat Transfer, 128(9), pp. 897-907.

[11] Annoni, M., 2012. Water jet velocity uncertainty in laser Doppler velocimetry measurements. Measurement, Volume 45, pp. 1639-1650.

[12] Anthony, D., 1990. The influence of a free surface on the development of turbulence in a submerged jet, s.l.: s.n.

[13] Arms, R. J. \& Hama, F. R., 1965. Localized induction concept on a curved vortex and motion of an elliptic vortex ring. The Physics of Fluids, 8(4), pp. 553-559.

[14] Auerbach, D. \& Grimm, T., 1994. Factors influencing the non-circular ring vortex motion. Meccanica, 29(4), pp. 351-359. 
[15] Barenghi, C. F., Donnelly, R. J. \& Vinen, W. F., 1983. Friction on quantized vortices in helium II. J. Low Temp. Phys., Volume 52, pp. 189-247.

[16] Bell, J. \& Mehta, R., 1998. Contraction design for small low-speed wind tunnels, Washington: National Aeronautics and Space Administration.

[17] Bera, J., Michard, M., Grosjean, N. \& Comte-Bellot, G., 2001. Flow analysis of two-dimensional pulsed jets by particle image velocimetry. Experiments in Fluids, Volume 31, pp. 519-532.

[18] Brunn, H. H., 1995. Hot wire anemometry : principles and signal analysis. s.1.:Oxford University Press.

[19] Cannelle, F. \& Amitay, M., 2007. Transitory behavior of a finite span synthetic jet. Physics of Fluids, Volume 19, pp. 1-15.

[20] Cater, J. \& Soria, J., 2002. The evolution of round zero-net-mass-flux jets. J. Fluid Mech., Volume 472, pp. 167-200.

[21] Cater, J., Soria, J. \& Lim, T., 2004. The interaction of the piston vortex with a piston-generated vortex ring. J. Fluid Mech., Volume 499, pp. 327-343.

[22] Chaudhari, M., Puranik, B. \& Agrawal, A., 2010. Effect of orifice shape in synthetic jet based impingement cooling. Experimental Thermal and Fluid Science, Volume 34, pp. 246-256.

[23] Chaudhry, I. A. \& Zhong, S., 2014. A single circular synthetic jet issued into turbulent boundary layer. Journal of Visualization, 17(2), pp. 101-111.

[24] Chaudhry, I. \& Zhon, S., 2012. Understanding the interaction of synthetic jet with the flat plate boundary layer. Trivendum, International Conference on Advanced Research in Mechanical Engineering.

[25] Choi, H. \& Williams, W. J., 1989. Improved time-frequency representation of multicomponent signals using exponential kernels. IEEE Transactions on acoustics, speech, and signal processing, 37(6), pp. 862-871.

[26] Cicca, G. \& Iuso, G., 2007. On the near field of an axisymmetric synthetic jet. Fluid Dynamics Research, Volume 39, pp. 673-693.

[27] Collis, D. C. \& Williams, M. J., 1959. Two dimensional convection from heated wires at low Reynolds number. Journal of Fluid Mechanics, 6(3), pp. 357-384.

[28] Crittenden, T.\& Glezer, A., 2006. A high speed, compressible synthetic jet. Physics of Fluids, 18(017107).

[29] Crook, A., Crowther, W. \& Wood, N., 2000. A parametric study of a synthetic jet in a cross flow, Manchester: ICAS, Congress. 
[30] Crook, A. \& Wood, N., 2001. Measurement and visualisations of synthetic jets. Nevada, s.n.

[31] da Silva, C. B. \& Metais, O., 2002. Vortex control of bifurcating jets: A numerical study. Physics of fluids, Volume 14, pp. 3798-3819.

[32] Danaila, I. \& Boersma, B. J., 2000. Direct numerical simulation of bifurcating jets. Physics of fluids, Volume 12, pp. 1255-1257.

[33] Dancova, P. \& Vit, T., 2008. Analysis of the synthetic jet. Journal of applied science in the thermodynamics and fluid mechanics, 2(1), pp. 1-9.

[34] Davies, T. \& Jackson, M., 1985. A procedure for the design of nozzles used for the production of turbulent liquid jets. Int. J. of heat and fluid flow, 6(4), pp. 298-305.

[35] Dhanak, M. R. \& de Bemardinis, B., 1981. The evolution of an elliptical vortex ring. J. Fluid Mech., Volume 109, pp. 189-216.

[36] Didden, N., 1979. On the formation of vortex rings: rolling up and production of circulation. Journal of Applied Mathematics and Physics, Volume 30, pp. 102-116.

[37] Duvigneau, R. \& Visonneau, M., 2006. Optimization of a synthetic jet actuator for aerodynamic stall control. Computers \& Fluids, Volume 35, pp. 624-638.

[38] Eibeck, R. A., Keller, J., Bramlette, T. \& Sailor, D., 1993. Pulse combustion: Impinging jet heat transfer enhancement. 94(1-6), pp. 147-165.

[39] Farge, B., Guezennec, Y., Ho, C. \& Meneveau, C., 1990. Continuous wavelet analysis of coherent structures. s.1., Center for Turbulence Research, pp. 331-338.

[40] Farge, M., 1992. Wavelet transforms and their applications to turbulence. Annu. Rev. Fluid Mech., Volume 24, pp. 395-457.

[41] Freund, J. B. \& Parviz Moin, P., 2000. Jet mixing enhancement by high-amplitude fluidic actuation. AIAA Journal, 38(10), pp. 1863-1870.

[42] Fung, P. \& Amitay, M., 2002. Active Flow Control Application on a Mini Ducted Fan UAV. Journal of Aircraft. 39(4), pp. 561-571.

[43] Gallas, Q., Holman, R., Raju, R. \& Mittal, R., 2004. Low dimensional modelling of zero-net-mass-flux actuators. Portland, s.n., pp. 1-12.

[44] Gao, L., Yu, S. C. .., Ai, J. J. \& Law, A. W. K., 2008. Circulation and energy of the leading vortex ring in a gravity-driven starting jet. Physics of Fluids, Volume 20, pp. 1-9.

[45] Gharib, M., Rambod, E. \& Shariff, K., 1998. A universal time scale for vortex ring formation. J. Fluid Mech., Volume 360, pp. 121-140. 
[46] Gilarranz, J. L., Traub, L. W. \& Rediniotis, O. K., 2005. A new class of synthetic jet acuators - part I: Design, fabrication and bench top characterization. Journal of Fluids Engineering, Volume 127, pp. 367-376.

[47] Ginette, S., Grossmann, A. \& Tchamitchian, P., 1998. Use of wavelet transforms in the study of propagation of transient acoustic signals across a plane interface between two homogeneous media. Inverse Problems and Theoretical Imaging, pp. 139-146.

[48] Glezer, A., 1998. The formation of vortex rings. Physics of Fluids, 31(12), pp. 35323542.

[49] Glezer, A. \& Amitay, M., 2002. Synthetic jets. Annu. Rev. Fluid Mech., Volume 34, pp. 503-529.

[50] Glezer, A., Amitay, M. \& Honohan, A., 2005. Aspects of low- and high-frequency actuation for aerodynamic flow control. AIAA Journal, 43(7), pp. 1501-1511.

[51] Gohil, T. B., Saha, A. K. \& Muralidhar, K., 2010. Control of flow in forced jets: a comparision of round and square cross sections.. Journal of visualization, Volume 13, pp. 141-149.

[52] Gorden, M., Cater, J. E. \& Soria, J., 2004. Investigation of the mean passive scalar field in Zero-net-mass-flux jets in cross-flow using planer-laser induced fluorescence. Physics of Fluids, 16(3), pp. 794 - 808.

[53] Gorden, M. \& Soria, J., 2002. PIV measurements of a zero-net-mass-flux jet in cross flow. Experiments in Fluids, Volume 33, pp. 863-872.

[54] Grinstein, F. F., 1995. Entrainment and transition to turbulence in subsonic rectangular jets. Reno, NV, 33rd Aerospace Sciences.

[55] Grinstein, F. F., 2001. Vortex dynamics and entrainment in rectangular free jets. $J$. Fluid mech., Volume 437, pp. 69-101.

[56] Grinstein, F. F., Gutmark, E. J. \& Parr, T., 1995. Near field dynamics of subsonic, free square jets. A computational and experimental study. Physics of Fluids, Volume 7, pp. 1483-1497.

[57] Heertch, 1976. Vortex formation in rectangular nozzles and umbildung in secondary vortices, s.1.: Max-Planck Institute for Fluid Dynamics.

[58] Hlawatsch, F., Manickam, T. G., Urabanke, R. L. \& Jones, W., 1995. Smoothed pseudo-Winger, Choi-Williams distribution, and cone-kernel representation: ambiguity-domain analysis and experimental comparison. Signal Processing, Volume 43, pp. 149-168. 
[59] Ho, C. M. \& Gutmark, E., 1987. Vortex induction and mass entrainment in a smallaspect-ratio elliptical jet. J. Fluid Mech., Volume 179, pp. 383-405.

[60] Ho, C. M. \& Huerre, P., 1984. Perturbed free shear layers. Annu. Rev. Fluid Mech., Volume 16, pp. 365-424.

[61] Holman, R. et al., 2005. Formation criterion for synthetic jets. AIAA Journal, 43(10), pp. 2110-2116.

[62] Hong, G. et al., 2002. Effectiveness of synthetic jets enhanced by instability of Tollmien-Schlichting waves. Missoury, s.n.

[63] Hussain, A. K. M. F., 1983. Coherent structures - reality and myth. Physics of Fluids, 26(10), pp. 2816-2850.

[64] Hussain, F. \& Husain, H. S., 1989. Elliptical jets, part 1: characterization of unexcited and excited jets. J. Fluid Mech., Volume 208, pp. 257-320.

[65] IIO, S. et al., 2006. Vortex behavior of a pulsating jet from rectangular nozzle. JSME International Journal, 49(4), pp. 988-994.

[66] Ingard, U. \& Labate, S., 1950. Acoustic circulation effects and the nonlinear impedance of orifices. Journal of the Acoustical Society of America, Volume 22, pp. 211-218.

[67] Jabbal, M., Wu, J. \& Zhong, S., 2006. The performance of round synthetic jets in quiescent flow. The Aeronautical Journal, pp. 385- 393.

[68] Jabbal, M. \& Zhong, S., 2008. The near wall effect of synthetic jets in a boundary layer. International Journal of Heat and Fluid flow, Volume 29, pp. 119 - 130.

[69] Jabbal, M. \& Zhong, S., 2010. Particle image velocimetry measurements of the interaction of synthetic jets with a zero-pressure gradient laminar boundary layer. Physics of Fluids, Volume 22, pp. 1-17.

[70] Jagadeesh, P., Murli, K. \& Idichandy, V., 2009. Experimental Investigation of Hydrodynamic Force Coefficients Over AUV Hull. Ocean Engineering, Volume 36, pp. 113-118.

[71] Jahanmiri, M., 2010. Active Flow Control: A Review, Goteborg, Sweden: Chalmers University of Technology.

[72] Jahanmiri, M., 2011. Excited jets and its application, Goteborg, Sweden: Division of Fluid Dynamics.

[73] James, R. D., Jacobs, J. W. \& Glazer, A., 1996. A round turbulent jet produced by an oscillating diaphragm. Phys. Fluid, 8(9), pp. 2484-2495. 
[74] Jiao Jian, A., Yu, S. C. M., Lawa, A. W.-K. \& Chua, L. P., 2005. Vortex Dynamics in Starting Square Water Jets. Physics of Fluids, 17(14106).

[75] Kanamori, A., Hiwada, M., Oyakawa, K. \& Senaha, I., 2011. Effect of orifice shape on flow behavior and impingement heat transfer. The Open Transport Phenomena Journal, Volume 3, pp. 9-16.

[76] Kareem, A. \& Gurley, K., 1999. Applications of wavelet transforms in eartquake, wind and ocean engineering. Engineering structures, Volume 21, pp. 149-167.

[77] Kelso, R. M., Lim, T. T. \& Perry, 1996. An experimental study of round jets in cross-flow. J. Fluid Mech., Volume 306, pp. 1111-144.

[78] Khamshah, N., Abdalla, A., Koh, S. \& Rashag, H., 2011. Issues and temperature compensation techniques for hot wire thermal flow sensors: A review. International Journal of the Physical Sciences , 6(14), pp. 3270-3278.

[79] Kim, C. \& Aggarwal, R., 2000. Wavelet transforms in power systems. Power Engineering journal, pp. 81-87.

[80] Kim, W., Kim, S., Choi, K. \& Kim, C., 2010. Experimental and computational study on flow characteristics by synthetic jets configuration. Orlando,Florida, 48th AIAA Aerospace Sciences Meeting, pp. 1-12.

[81] Kiya, M., Toyoda, K., Ishii, H. \& Ohe, T., 1992. Numerical simulation and flowvisualization experiment. Fluid Dynamics Research, Issue 10, pp. 117-131.

[82] Krieg, M. \& Mohseni, K., 2008. Thrust characterization of a bio inspired vortex ring thruster for locomotion of underwater robots. IEEE Journal of Oceanic Engineering, 33(2), pp. 123-132.

[83] Krieg, M. \& Mohseni, M., 2010. Dynamic modeling and control of biologically inspired vortex ring thrusters for underwater robot locomotion. IEEE Transactions of Robotics , 26(3), pp. 542-554.

[84] Krieg, M., Pitty, A., Salehi, M. \& Mohseni, K., 2005. Optimal thrust characteristics of a synthetic jet actuator for applicaion in low speed maneuvering of underwater vehicle, s.1.: s.n.

[85] Krothapalli, A., Baganoff, D. \& Karamcheti, K., 1981. On the mixing of a rectangular jet. J. Fluid Mech., Volume 107, pp. 201-220.

[86] Krueger, P. S., 2003. Vortex ring pinch off in the presence of simultaneously initiated uniform background co-flow. Physics of Fluids, 14(7), pp. L49- L52.

[87] Krueger, P. S., 2005. An over-pressure correction to the slug model for vortex ring circulation. J. Fluid Mech., Volume 545, pp. 427-443. 
[88] Krueger, P. S. \& Gharib, M., 2003. The significance of vortex ring formation to the impulse and thrust of a starting jet. Physics of Fluids, Volume 15, pp. 1271-1281.

[89] Krueger, P. S. et al., 2008. Vortex rings in bio-inspired and biological jet propulsion. Advances in Science and Technology, Volume 58, pp. 237-246.

[90] Kumar, A., Gupta, M., Saha, A. K. \& Panigrahi, P. K., 2014. "Effect of free surface on submerged synthetic jet parallel to the surface. Kanpur, s.n.

[91] Kumar, A., Gupta, M., Saha, A. K. \& Panigrahi, P. K., 2014. Flow characteristics of synthetic jet on torpedo shape model in cross flow. Kanpur, s.n.

[92] Kumar, A. et al., 2010. Combined experimental and numerical study of synthetic jet in quiescent flow. Chennai, s.n.

[93] Lardeau, S. \& Leschziner, M. A., 2011. The interaction of round synthetic jets with a turbulent boundary layer separating from a rounded ramp. J. Fluid Mech., Volume 638, pp. 172-211.

[94] Lee, C., Hong, G., Ha, Q. P. \& Mallinson, S. G., 2003. A piezoelectrically actuated micro synthetic jet for active flow control. Sensors and Actuators, Volume A 108, pp. 168-174.

[95] Liddle, S. C. \& Wood, N. J., 2005. Investigation into the clustering of synthetic jet actuators for flow separation control applications. The Aeronautical Journal, pp. 35 $-44$.

[96] Linden, P. F. \& Turner, J. S., 2001. The formation of 'optimal' vortex rings, and the efficiency of propulsion devices. J. Fluid Mech., Volume 427, pp. 61-72.

[97] Linden, P. F. \& Turner, J. S., 2004. Optimal vortex rings and aquatic propulsion mechanisms. The Royal society, Volume 271, pp. 647-653.

[98] Mallinson, S. G., Riezes, J. A., Hong, G. \& Westbury, P. S., 2004. Analysis of hotwire anemometry data obtained in a synthetic jet flow. Experimental Thermal and Fluid Science, Volume 28, pp. 265-272.

[99] Maxworthy, T., 1977. Some experimental studies of vortex rings. J. Fluid Mech., 81(3), pp. 465-495.

[100] Melande, M. V. \& Hussain, F., 1989. Cross-linking of two antiparallel vortex tubes. Physics of Fluids, 1(4), pp. 633-636.

[101] Melis, M. D. et al., 2009. Blood pressure waveform analysis by means of wavelet transformation. Med Biol Eng Comput, Volume 47, pp. 165-173.

[102] Milanovic, I. \& Zaman, K., 2005. Synthetic jet in cross flow. AIAA, 43(5), pp. 929940. 
[103] Mittal, R. \& Rampunggon, P., 2002. On the virtual aeroshaping effect of synthetic jets. Physics of Fluids, 14(4), pp. 1533-1536.

[104] Mittal, R., Rampunggoon, P. \& Udaykumar, H. S., 2001. Interaction of a synthetic jet with a flat plate boundary layer. AIAA, pp. $1-11$.

[105] Mohseni, K., 2006. Pulsatile vortex generators for low-speed. Ocean Engineering, Volume 33, p. 2209-2223.

[106] Mohseni, K. \& Gharib, M., 1998. A model for universal time scale of vortex ring formation. Physics of Fluids, Volume 10, pp. 2436-2438.

[107] Mohseni, K., Ran, H. \& Colonius, T., 2001. Numerical experiments on vortex ring formation. J. Fluid Mech. , Volume 430, pp. 267-282.

[108] Narasimha, R., 2007. Wavelet dignostics for detection of coherent structures instantaneous turbulent flow imagery: A review. Sadhana, Volume 32, pp. 29-42.

[109] Nitsche, M., 2006. Vortex dynamics, s.1.: Elsevier Science Ltd.

[110] Oshima, Y., Izutsu, N. \& Oshima, K., 1988. Bifurcation of an elliptical vortex ring. Fluid Dynamics Research, Volume 3, pp. 133-139.

[111] Parekh, D. E., Leonard, A. \& Reynold, W. C., 1988. Bifurcating jets at high Reynolds number, Thermosciences Division, Department of Mechanical Engineering, Stanford University, Stanford, CA: s.n.

[112] Pavlova, A. \& Amitay, M., 2006. Electronic cooling using synthetic jet impingement, s.1.: Rensselaer Polytechnic Institute.

[113] Pavlova, A. A., Otani, K. \& Amitay, M., 2008. Active control of sprays using a single synthetic jet actuator. International Journal of Heat Flow, Volume 29, pp. 131-148.

[114] Prince, S. A., Khodagolian, V. \& Gaind, R., 2012. An experimental study of a pulsed air jet and an acoustic jet on a low speed turbulent boundary layer. Brisbane, AU, 28 th International congress of the Aeronautical sciences.

[115] Qayoum, A., Gupta, V. \& Panigrahi, P., 2009. Turbulent Boundary Layer Manipulation using Synthetic Jet Actuation. London, U.K., s.n.

[116] Qayoum, A., Gupta, V., Panigrahi, P. K. \& Murlidhar, K., 2010. Influence of amplitude and frequency modulation on flow created by a synthetic jet actuator. Sensors and Actuators A: Physical, Volume 162, pp. 36-50.

[117] Raju, R., Mittal, R., Gallas, Q. \& Cattafesta, L., 2005. Scaling of vorticity flux and entrance length effects in zero-net-mass-flux. AIAA, pp. 1-13. 
[118] Rathnasingham, R. \& Breuer, K. S., 1997. Coupled fluid-structural characteristics of actuators for flow control. AIAA, 35(5), pp. 832-837.

[119] Rathnasingham, R. \& Breuer, K. S., 2003. Active control of turbulent boundary layers. J. Fluid Mech., Volume 495, pp. 209-233.

[120] Reynolds, W. C., Parekh, D. E., Juvet, P. J. D. \& Lee, M. J. D., 2003. Bifurcating and blooming jets. Annu, Rev. Fluid Mech., Volume 35, pp. 295-315.

[121] Rioul, O.\& Vetterli, M., 1991. Wavelets and signal processing. IEEE SP Magazine, pp. 14-38.

[122] Ritchie, B. D., Majumdar, D. R. \& Seitzman, J. M., 2000. Mixing in coaxial jets using synthetic jet actuators. AIAA, Volume 0404, pp. 1-8.

[123] Rosenfeld, M., Rambod, E. \& Gharib, M., 1998. Circulation and formation number of laminar vortex rings. J. Fluid Mech., Volume 376, pp. 297-318.

[124] Sadiku, M. N. O., Akujuobi, C. M. \& Garcia, R. C., 2005. An introduction to wavelets in electromagnetics. IEEE microwave magazine, pp. 63-72.

[125] Sahambi, J. S. T. S. N. \& Bhatt, R. K. P., 1997. Using wavelet transforms for ECG characterization. IEEE Engineering in medicine and biology, pp. 77-83.

[126] Sahni, O., Wood, J., Jansen, K. E. \& Amitay, M., 2010. 3-D Interactions between a finite-span synthetic jet and a cross-Flow. Journal of Fluid Mechanics.

[127] Sailor, D. J., Rohli, D. J. \& Fu, Q., 1999. Effect of variable duty cycle flow pulsations on heat transfer enhancement for an impinging air jet. International Journal of Heat and Fluid Flow, 20(6), pp. 574-580.

[128] Sau, A., Sheu, T. W. H., Hwang, R. R. \& Yang, W. C., 2004. Three dimensional simulation of square jets in cross-flow. Physical Review, Volume E 69.

[129] Schadowk, K. C., Wilson, K. J. \& Lee, M. J., 1984. Enhancement of mixing in ducted rockets with elliptical gas-generator nozzles. Ohio, AiAA.

[130] Schram, C. \& Riethmuller, M. L., 2001. Vortex ring evolution in an impulsively started jet using digital particle image velocimetry and continuous wavelet analysis. Measurement science and technology, Volume 12, pp. 1413-1421.

[131] Sharma, R. N., 2007. Some insights into synthetic jet actuation from analytical modelling. Gold Coast, Australia, s.n., pp. 1242-1248.

[132] Shusser, M. \& Gharib, M., 2000. Energy and velocity of a forming vortex ring. Physics of Fluids, 12(3), pp. 618-621.

[133] Shuster, J. M. \& Smith, D. R., 2007. Experimental study of the formation and scaling of a round synthetic jet. Physics of Fluids, Volume 19, pp. 1-21. 
[134] Shuster, J., Pink, R., McEligot, D. \& Smith, D., 2005. The interaction of a circular synthetic jet with a cross-flow boundary layer. Toronto, Canada, s.n., pp. 1-16.

[135] Siekmann, J., 1962. On a pulsating Jet from the end of a tube, with application to the propulsion of certain aquatic animals. Journal of Fluid Mechanics, 15(3), pp. 399-418.

[136] Smith, B. L. \& Glazer, A., 1998. The formation and evolution of synthetic jets. Physics of Fluids, Volume 10, pp. 2281- 2297.

[137] Smith, B. L. \& Glezer, A., 2002. Jet vectoring using synthetic jets. J. Fluid Mech., Volume 458, pp. 1-34.

[138] Smith, B. L. \& Swift, G. W., 2001. Synthetic jets at large Reynolds number and comparison to continuous jets. AIAA, pp. 1-19.

[139] Smith, B. L. \& Swift, G. W., 2003. A comparison between synthetic jets and continuous jets. Experiments in Fluids, Volume 34, pp. 467-472.

[140] Sullivan, I. S. et al., 2008. Dynamics of thin vortex rings. J. Fluid Mech., Volume 609, pp. 319-347.

[141] Tang, H. \& Zhong, S., 2005. Incompressible flow model of synthetic jet actuators. AIAA, 44(4), pp. 908-912.

[142] Tesar, V. \& Kordik, J., 2011. Spectral analysis of a synthetic jet. Sensors and Actuators, 167(2), pp. 213-225.

[143] Thomas, A. P., Burdick, J. \& Mohseni, K., 2005. An experimental study of voicecoil driven synthetic jet propulsion for underwater vehicles. s.1., s.n., pp. 923-927.

[144] Thomas, A. P. et al., 2005. Synthetic jet propulsion for small underwater vehciles, California 91125: s.n.

[145] Trentacoste, N. \& Sforza, P., 1967. Further experimental results for three dimensional free jets. AIAA , 5(5), pp. 885- 891.

[146] Uchiyama, H. \& Ide, M., 2009. Development of a hot-film anemometer calibrator for water measurement. Flow Measurement and Instrumentation, Volume 20, pp. 75-80.

[147] Utturkar, Y. et al., 2003. A jet formation criterion for synthetic jet actuators. Reno, NV, s.n., pp. 1-9.

[148] Utturkar, Y., Mittal, R., Rampunggoon, P. \& Cattafesta, L., 2002. Sensitivity of synthetic jets to the design of the jet cavity. Reno, NV, s.n., pp. 1-8.

[149] Wakefield, G. H. \& Pielemeier, W. J., 1992. Time-frequency and time-scale analysis for musical transcription. IEEE-SP International Symposium, pp. 421-424. 
[150] Wang, J., Shan, R., Zhang, C. \& Feng, L., 2010. Experimental investigation of a novel two-dimensional synthetic jet. European Journal of Mechanics Bio-Fluids, Volume 29, pp. 342-350.

[151] Watson, M., Jaworski, A. J. \& Wood, N. J., 2003. A study of synthetic jets from rectangular and dual-circular orifices. The Aeronautical Journal, pp. 427-434.

[152] Webster, D. R. \& Longmire, E. K., 1998. Vortex rings from cylinders with inclined exits. Physics of Fluids, Volume 10, pp. 400-416.

[153] Wen, X. \& Tang, W., 2012. Interaction of synthetic jets with laminar and turbulent boundary layers. Launceston, s.n.

[154] Wu, K. E. \& Breuer, K. S., 2003. Dynamics of synthetic jet actuator arrays for flow control. Orlando, Florida, s.n., pp. 1-8.

[155] Yao, Z. \& Xungang, S., 1997. Evolution of single elliptical vortex rings. ACTA MECHANICA SINICA, 13(1), pp. 17-25.

[156] Yu, S. C. M., Law, A. W. \& Aj, J. J., 2007. Vortex formation process in gravitydriven starting jets. Exp. Fluids, Volume 42, pp. 783-797.

[157] Zaman, K. B., 1996. Axis switching and spreading of an asymmetric jet: the role of coherent structure dynamics. J. Fluid Mech., Volume 316, pp. 1-27.

[158] Zaroodny, S. J., 1966. Revised theory of vortex rings - A simplified review of the state-of-art, Maryland: U.S. Army Limite War Laboratory.

[159] Zhong, S., Garcillan, L. \& Wood, N. J., 2005. Dye visulisation of inclined and skewed synthetic jets in a cross flow. The Aeronautical Journal, pp. 147-155.

[160] Zhong, S., Millet, F. \& Wood, N. J., 2005. The behavior of circular synthetic jets in a laminar boundary layer. The Aeronautical Journal, pp. 461- 470.

[161] Zhou, J., Tang, H. \& Zhong, S., 2009. Vortex roll-up criteria for synthetic jets. AIAA Journal, 47(5), pp. 1252-1263.

[162] Zhou, J. \& Zhong, S., 2010. Coherent structures produced by the interaction between synthetic jets and a laminar boundary layer and their surface shear stress patterns. Computers \& Fluids, Volume 39, pp. 1296-1313.

[163] Zulkifli, R., Sopian, K., Abdullah, S. \& Takriff, M., 2009. Correlations between local Nusselt number and Reynolds number for steady and pulsating circular jet. International Journal of Engineering and Technology, 6(1), pp. 9-20.

[164] Zumbrunnen, D. A. \& Aziz, M., 1993. Convective heat transfer enhancement due to intermittency in an impinging Jet. J. Heat Transfer, 115(1), pp. 91-99. 
[165] Zumbrunnen, D. A. \& Aziz, M., 2009. Alterations to coherent flow structures and heat transfer due to pulsations in an impinging air jet. International Journal of Thermal Sciences, 6 (1), pp. 236-248. 


\section{Appendix A}

\section{Vortex formation and effect of dimensionless numbers}

\section{A.1 Vortex formation and propagation}

The formation of vortex ring at the nozzle exit begins at the sharp edge, where the shear layer leaves the solid boundary and the combined effect of positive and negative vortices present at the tip makes the shear layers roll in outward direction shown Figure A.1 (a). Subsequently, the vortex ring propagates away from the jet exit due to its self-induced velocity (Maxworthy, 1977). A small increases in the trajectory of vortex tube occurs near the jet exit during Figure A.1 (a) because as the vortex ring detached itself from the orifice, its size decreases slightly to overcome the drag of following suction stroke. This phenomenon is termed as vortex overshoot. When the supply of energy from the feeding shear layer is less in comparison to the circulation energy of the forming vortex ring or leading vortex ring, vortex ring becomes saturated and stops receiving any further fluid coming out from the orifice. The fluid rejected by the leading vortex forms secondary vortex ring which is known as trailing jet. Akhmetov and Kisarov (1966) working on vortex ring suggests three types of streamlines inside the vortex ring: $\psi<0, \psi=0$ and $\psi$ $>0$ as illustrated in Figure A.1 (c), where $\psi$ is streamline function. All the streamlines inside the vortex ring where $\psi<0$ are closed with high stability and vorticity at the center 
of vortex tube. The region having streamlines as $\psi=0$ separates the positive and negative streamline region. The region in which $\psi>0$, streamlines are open and unstable in nature due to low vorticity. In results, the surrounding fluid interact with them causing vortex ring to grow in size by losing energy. Due to continuous entrainment of surrounding fluid, more energy loss occurs due to viscous dissipation. When the remaining circulation energy of vortex ring are unable to hold the growing size of vortex ring, it breaks into smaller vortices.

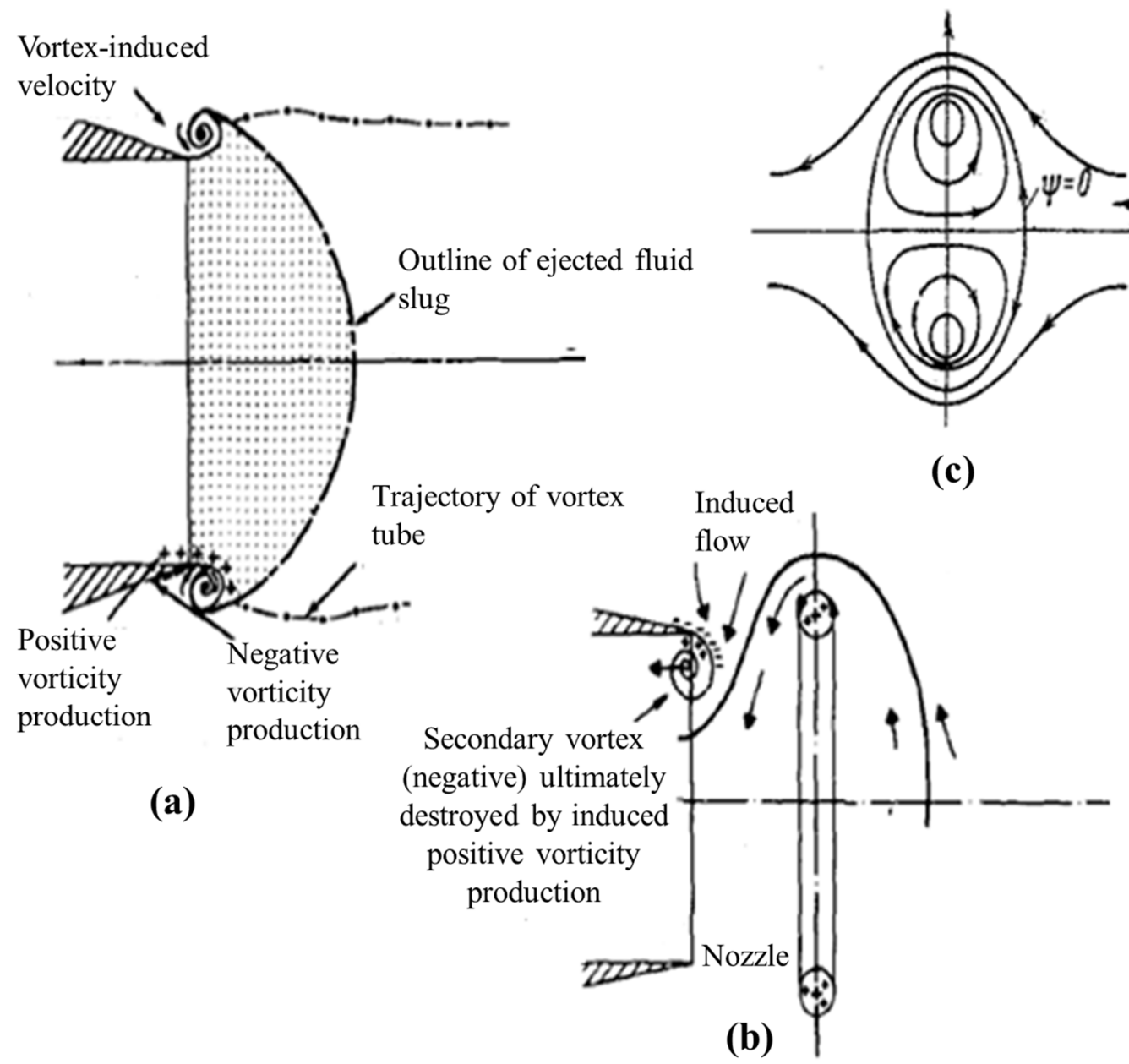

Figure A.1: (a) Shear layer roll up at nozzle exit during expulsion period, (b) flow of ambient fluid inside the cavity by induced flow during vortex detachment process (Maxworthy, 1997) and (c) the streamline distribution for an ideal isolated vortex ring (Akhmetov \&. Kisarov,1966) 
The formed vortex ring initiates forward motion through its self- induced velocity as soon as it detaches itself from the nozzle or orifice as shown in Figure A.2 (a). In the case of synthetic jet, since each ejection period is followed by a suction period, there is a possibility that ejected fluid may be fully or partially sucked back inside the cavity. If the induced velocity is sufficient to overcome the suction effect of following suction stroke, the vortex ring does not digested back inside the cavity and continues to move in downstream direction.

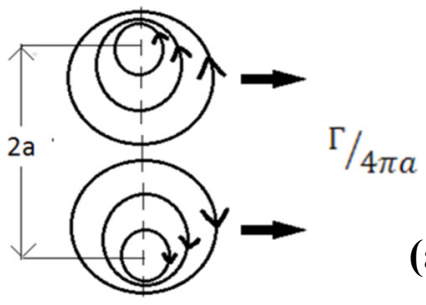

(a)

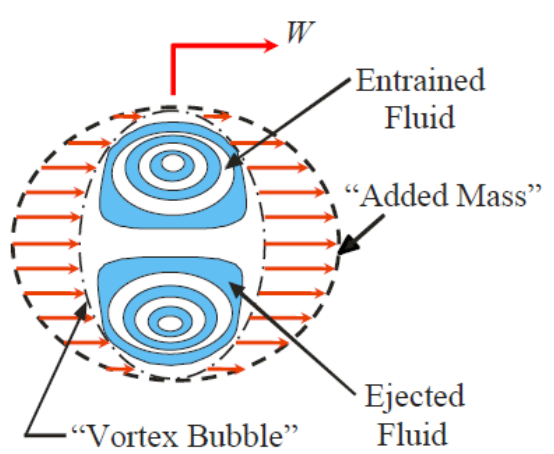

(b)


A: Vortex Bubble
B: Braid vortices
$\mathrm{C}$ : Entrained fluids
D: Irrotational fluids
E: Ejected fluids

(c)

Figure A.2: (a) Velocity due to rolling of vortex rings (b) mass distribution for an isolated vortex ring (Krueger \& Gharib, 2003) and (c) mass distribution of a synthetic jet (Yu et al., 2007)

As the vortex ring moves further, surroundings fluids are dragged or pushed in different way. Krueger and Gharib (2003) suggested that the whole mass of the vortex ring can be divided into three classes of accelerated fluid as depicted in Figure A.2 (b). They are: (i) the ejected fluid from the cavity during expulsion period, (ii) the dragged ambient fluid inside the vortex ring bubble which looks like circular rings sandwiched between the layers of ejected mass and (iii) added mass in front of vortex ring which is pushed forward by the moving vortex ring. Yu et al. (2007) also categorizes the total fluid mass 
of synthetic jet with a trailing jet into six different types as shown Figure A.2 (c). The irrotational fluid between the rotating ring and braid vortices region is absent in the model of Krueger and Gharib (2003). The momentum associated with masses of different types is studied by Krueger et al. (2008) using a robosquid. They showed that vortex rings with short pulse without trailing jet is more efficient than the long pulse having trailing jet for thrust maximization. They have also reported that the pulsed jet produces 2-3 times higher thrust as compared to the continuous jet. Another finding is that the total mass of moving vortex ring is the summation of all these masses and the propagation, breaking and dissipation of vortex ring depends on how these masses interact among themselves. Since the presence of trailing jet affects the added as well as the dragged mass, the distribution and interaction of different masses is not same for synthetic jets having trailing jet and without trailing jet condition.

\section{A.2 Dimensional analysis of synthetic jet}

Dimensional analysis is a method of reducing complex physical problems to the simplest form using the concept of similarity prior to obtaining a quantitative answer. The magnificence of the dimensional analysis lies in the fact that the cumulative effect of two or more parameters combined in a single or more non-dimensional numbers. The various parameters, which govern the formation and propagation of synthetic jet along with the corresponding influence on the flow properties, are given below.

Geometrical parameters:

- orifice diameter(D) : overall ejection and suction velocity

- orifice thickness(h): formation of shear layer

- orifice shape: velocity distribution at orifice exit, curvature effect on vortex ring

- cavity height $(\mathrm{H})$ and diameter $\left(\mathrm{D}_{\mathrm{c}}\right)$ : compressibility of fluid at high Reynolds number, cavitation formation inside the cavity 
Flow parameters:

- actuator displacement $(\Delta)$ : controls amount of fluid displaced

- actuator frequency(f): fluid ejection and suction properties

- crossflow velocity $\left(\mathrm{U}_{\infty}\right)$

Fluid properties:

- $\operatorname{density}(\rho)$ : momentum and force

- $\quad \operatorname{viscosity}(v)$ : shear layer formation and viscous dissipation

The synthetic jet velocity is not included because it is included in terms of actuator amplitude $(\Delta)$, frequency (f) and orifice shape. For deriving the non-dimensional terms using Buckingham- $\pi$ theorem, out of nine variables three basic $\pi$ terms $D$, f and $\rho$ are chosen. The $\pi$ theorem reductions leads to 6 non-dimensional quantities given below:

$$
\begin{aligned}
& \pi_{1}=\mathrm{fD}^{2} / \nu \\
& \pi_{2}=\Delta / D \\
& \pi_{3}=D_{c} / D \\
& \pi_{4}=H / D \\
& \pi_{5}=t / D \\
& \pi_{6}=f D / U_{\infty}
\end{aligned}
$$

The square root of $\pi_{1}$ multiplied by $2 \pi$ gives Stokes Number (S), which gives the information regarding the fluctuating particle response time to the characteristic fluid time scale as shown in equation A.7

$$
S=\sqrt{\left(2 \pi f D^{2}\right) / v}
$$


Reynolds number is most frequently defined based on the actuator orifice diameter (d) and spatial and time-averaged jet velocity during an expulsion stroke, function of $\pi_{1}, \pi_{2}$ and $\pi_{3}$.

$$
R e=\left(\bar{U}_{o} d\right) / v=f\left(\pi_{1}, \pi_{2}, \pi_{3}\right) \quad, \text { where } \quad \bar{U}_{o}=\frac{1}{t} \int_{0}^{t} u_{p}(t) d t
$$

One of the most important and widely used parameter in the field of synthetic jet is "formation number" which is equal to the reciprocal of Strouhal number $\left(\mathrm{S}_{\mathrm{t}}\right)$

$$
1 / S_{t}=R_{e} / s^{2}=\left(0.25 * \pi_{1} * \pi_{2} * \pi_{3}\right) / \sqrt{2 * 3.1415 * \pi_{1}}=f\left(\pi_{1}, \pi_{2}, \pi_{3}\right)
$$

In the cross-flow the two non-dimensional numbers, velocity ratio (VR) and jet momentum co-efficient $\left(\mathrm{C}_{\mu}\right)$ are also comes into the picture.

$$
\begin{aligned}
& V R=\bar{U}_{o} / U_{\infty}=\frac{1}{4} * \pi_{2} * \pi_{6} *\left(\pi_{3}\right)^{2}=f\left(\pi_{4}, \pi_{5}, \pi_{6}\right) \\
& C_{\mu}=2 *\left(h / D_{o}\right) *\left(\bar{U}_{o} / U_{\infty}\right)^{2}=2 * \pi_{5} *(V R)^{2}=f\left(\pi_{2}, \pi_{3}, \pi_{5}, \pi_{6}\right)
\end{aligned}
$$

Jabbal et al.(2006) given three non-dimensional number through parametric analysis and established relationship between these non-dimensional numbers with the Reynolds number and slug length which is and given as

$$
\overline{\mathrm{Q}}_{0} /_{(\rho \nu \mathrm{D})} \propto \mathrm{R}_{\mathrm{e}}, \quad \overline{\mathrm{M}}_{0} /_{\left(\rho v^{2}\right)} \propto \mathrm{R}_{\mathrm{e}}{ }^{2} \text { and } \quad \Gamma_{0} / \nu \propto \mathrm{R}_{\mathrm{e}} \cdot \mathrm{L}
$$

Where $\overline{\mathrm{Q}}_{0}$ and $\overline{\mathrm{M}}_{0}$ is time-averaged mass flow rate and momentum and $\Gamma_{0}$ is total circulation. 


\section{A.2.1 Formation number}

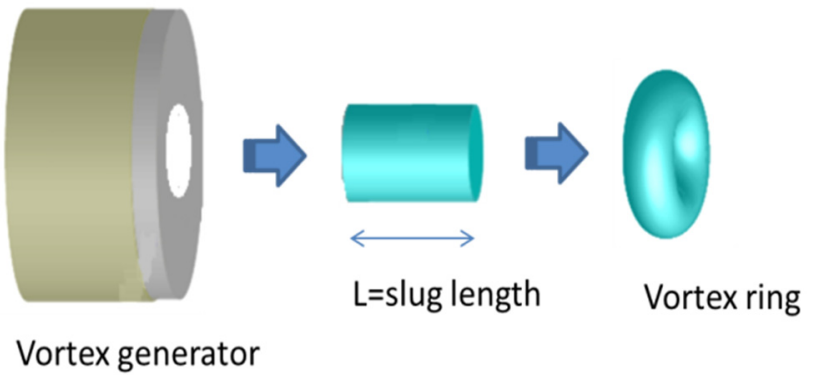

Figure A.3: Slug model assumptions in vortex formation

$$
\text { slug length }=L=\int_{0}^{t} u_{p}(t) d t
$$

Where $u_{\mathrm{p}}(\mathrm{t})$ is instantaneous jet exit velocity at the center of orifice. Slug length is a nondimensional number related to the vortex ring formation and its propagation nearer to the jet exit. It gives an estimate of energy injected into the environment by synthetic jet during expulsion period.

Formation number $=$ slug length/orifice diameter $=L / D=1 / S_{t}$

Here the formation number $(\mathrm{L} / \mathrm{D})$ is based on the slug length, which is defined as equivalent length of the fluid column ejected out from the orifice or nozzle during expulsion period having cross-section area of orifice as shown in Figure A.3 In this model, it is assumed that vortex ring is outcome of the same amount of fluid present in the slug coming out from the orifice. The other assumption is radial component of velocity gradient is neglected. The lowest value of $\mathrm{L} / \mathrm{D}$ equal to 1.0 , was close to the limiting synthetic jet formation condition identified by Holman et al (2005) At values lower than this, the ejected fluid through the orifice is ingested back in the following suction period (Fushui Guo, 2010). 


\section{A.2.2 Reynolds number}

Proper balance between the inertia and viscous forces (Reynolds number) and between unsteady and inertia forces (Strouhal number) is required to generate synthetic jet of highest coherence level and directivity for effective flow control (Wu \& Breuer (2003)). Fushui Guo (2010) found that isolated vortex rings could not be produced for $\mathrm{L} / \mathrm{D}<0.4$. Crook and Wood (2001) presented the effect of Reynolds number and L/D ratio on circular synthetic jet using flow visualization as shown in Figure A.4.

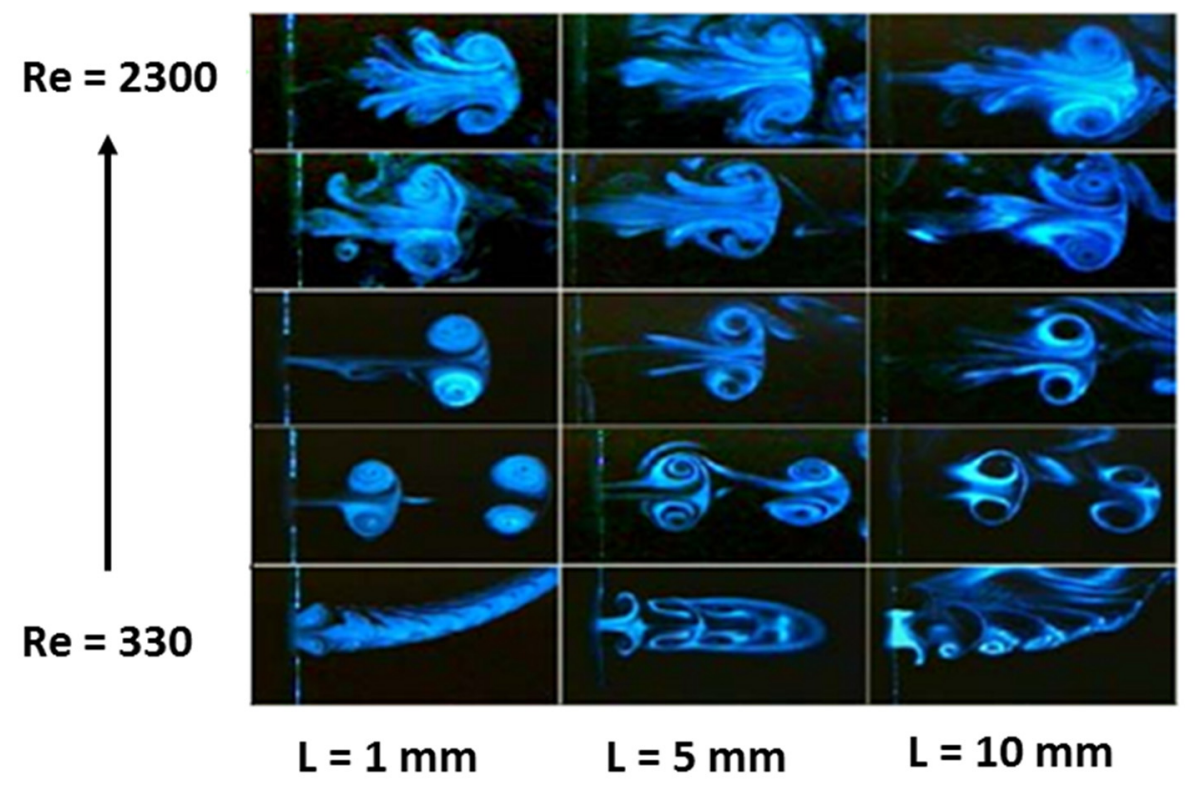

Figure A.4: Effect of Reynolds number at three different value of slug length (Crook and Wood, 2001)

\section{A.2.3 Strouhal number}

Strouhal number is defined as the ratio of inertia forces (caused by local unsteady acceleration) to the inertia forces (caused by the convective acceleration over a region in the neighborhood). In synthetic jet, this number compares the excitation frequency to the amount of time it would take an element of fluid to pass through the orifice. A sudden decrease in circulation is observed at high Strouhal number, indicating that the presence of the preceding pulse does not eliminate the pinch off even when the pulse are very close 
(Kruger, 2001). A synthetic jet is generally characterized by the movement of large-scale coherent structures, which under certain circumstances may disintegrate into smaller vortices. The mutual interaction of the vortex rings, which depending on the value of the Strouhal and the Reynolds numbers could lead to the expulsion of a synthetic jet with incoherent structures (Shuster \& Smith, 2007)

\section{A.2.4 Stokes number}

The Stokes number is the ratio between the unsteady force and viscous force in an oscillating flow. Rathnasingham and Breuer (1997) stated that, the Stokes number characterizes viscous effects of fluid for orifice of a finite length. At lower value of stokes number, the oscillating boundary layer dominates the entire orifice duct and chock the flow. In this condition, the jet velocity is proportional to the area of the orifice and exhibits a parabolic shape velocity profile. At higher value of stokes number the boundary layer is confined close to the orifice wall and if the flow is considered to be inviscid, the jet velocity is inversely proportional to the area of the orifice. In this case, the velocity profile exhibits a top hat shape. The velocity peak stays at the center of the orifice exit when $\mathrm{S}<10$ and the leading edge of the vortex sheet is unable to curve due to the axisymmetric and continuum of the flow. The maximum velocity gradually retreats towards the orifice wall as Strokes number increase and when $\mathrm{S}>10$, a potential core appears at the center of the orifice, allowing the rollup of vortex rings (Zhou et. al., 2009). The effect of Stokes number on the evolution of vortex formation is shown in Figure A.5. 
(a)

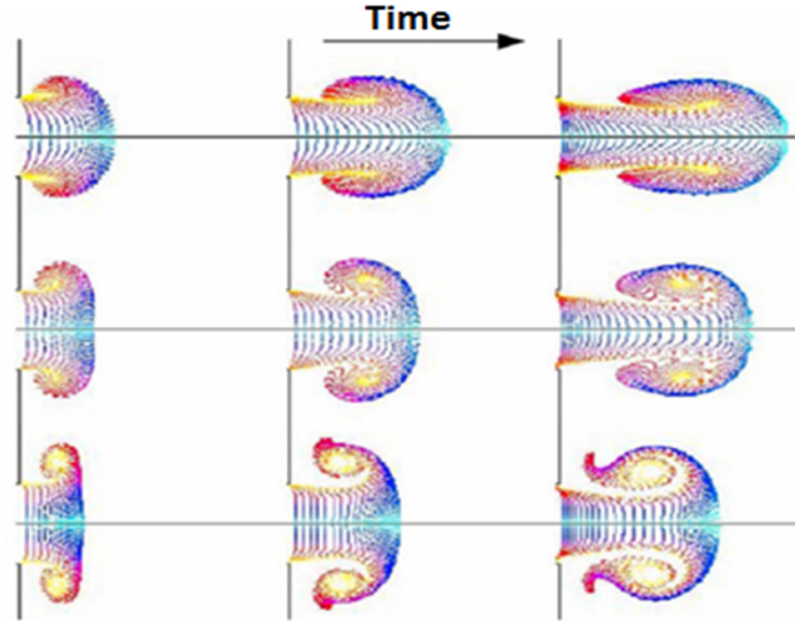

Figure A.5: Effect of Stokes number on vortex formation at (a) $S=7$, (b) $S=12.1$ and (c) $S=22.2$ having dimension less stroke length $\left(\mathrm{L} / \mathrm{D}_{\mathrm{h}}\right)$ equal to 3.24 (Zhou et. al., 2009). 


\section{Appendix-B}

\section{Instrumentation \& facility}

\section{B.1 Common peripheral devices}

The brief details of key instrument used in experimental set-up are discussed below.

\section{B.1.1 Camera}

A CMOS camera Basler ${ }^{\circledR} 501 \mathrm{~K}$ (see Figure B.1) having maximum frame grabbing rate of 74 fps and $1280 \times 1024$ pixel per inch of resolution is used for images acquisition of flow field. The quantum efficiency of the camera is $515 \mathrm{~nm}$ approximately.
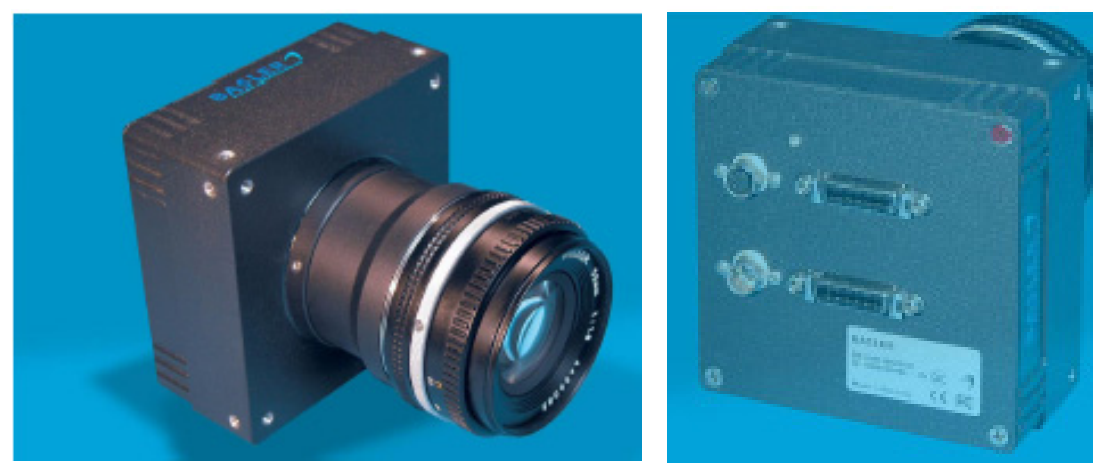

Figure B.1: Image of the Basler® ${ }^{\circledR}$ 501K CMOS camera 


\section{B.1.2 3-D Traverse for hot-film positioning}

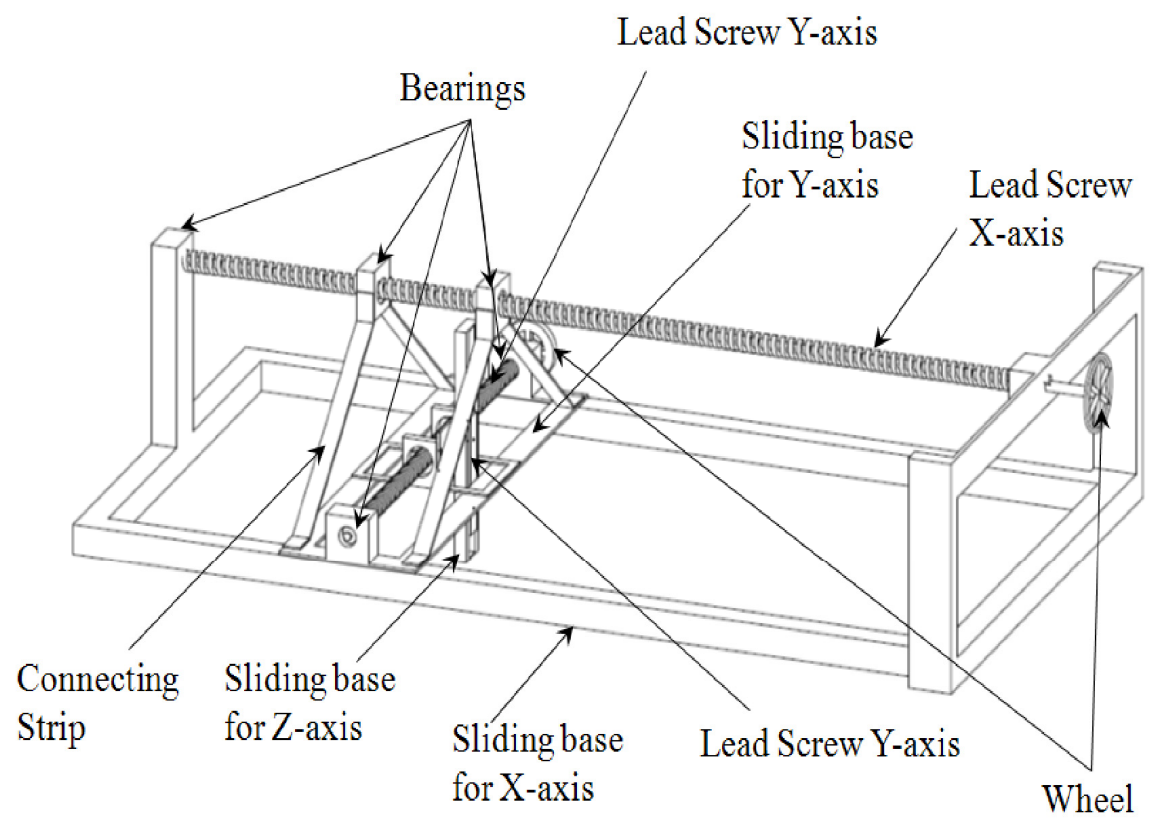

(a)

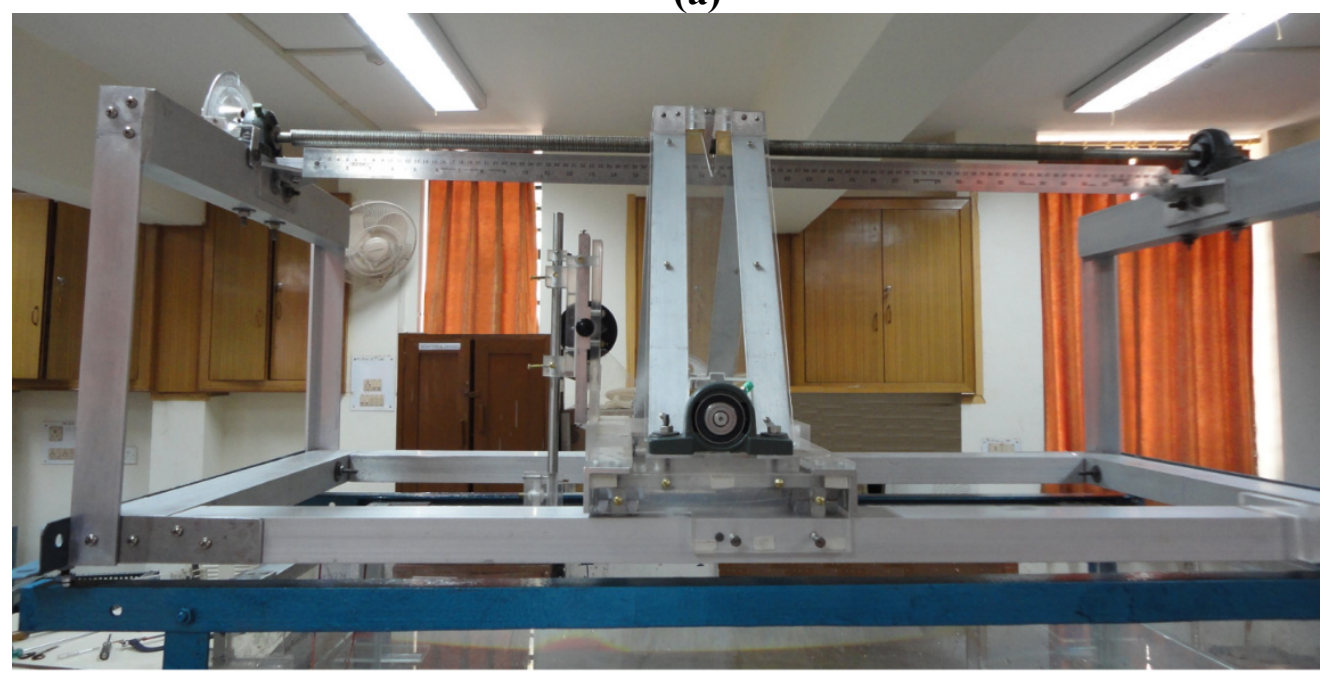

(b)

Figure B.2: (a) Design sketch and (b) actual image of 3-dimensional traverse for mounting hotfilm sensor.

A 3-Dimensional traverse mechanism is designed and fabricated to locate the hot-film probe precisely at any desired location in the measurement area (see Figure B.2 (a)). The traverse mounted on the top of test section is shown in Figure B.2 (b). The maximum dimensions over which traverse can move are $800 \mathrm{~mm}$ in $\mathrm{X}$-direction, $360 \mathrm{~mm}$ in $\mathrm{Y}$ direction and $400 \mathrm{~mm}$ in Z-direction. Hand wheels are used to rotate the lead screw with 
pointer showing the angle of rotation. The least count of all three protractors is $1^{\circ}$ that gives a resolution equal to $0.01 \mathrm{~mm}$ in $\mathrm{X}, \mathrm{Y}$ and $\mathrm{Z}$-directions.

\section{B.1.3 Articulated arm}

The Laser source and necessary optics used for light sheet formation is shown in Figure

B.3. The laser light sheet is guided to the articulated arm by levelling the launching mount with the help of levelling screw. A thin laser sheet $(\sim 1.0$ to $1.5 \mathrm{~mm})$ is produced from a beam of laser light using a concave lens having focal length of $-15 \mathrm{~mm}$. The tripod facilitates additional movement of laser sheet. The maximum movement of the articulated arm is limited by its two hinged arms each having length of $1 \mathrm{~m}$.

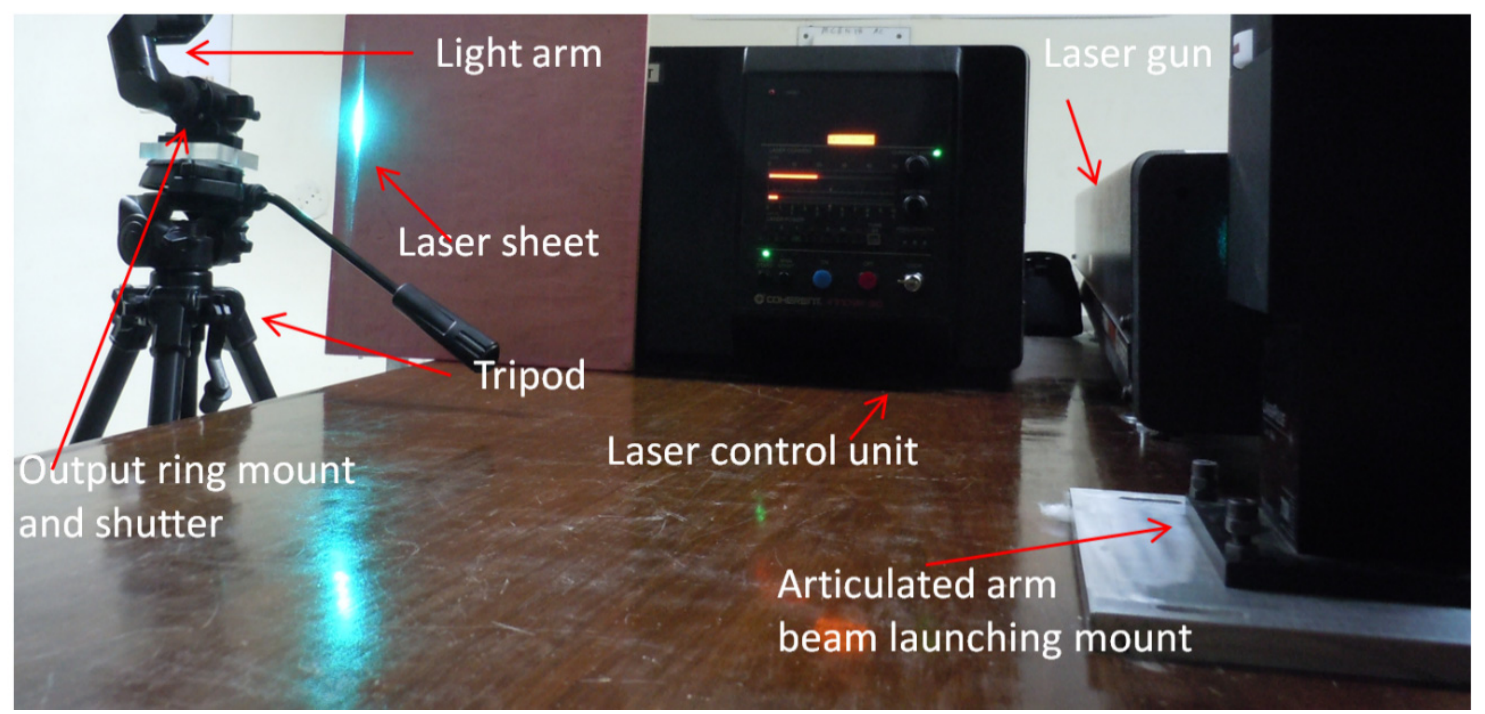

Figure B.3: Laser sheet with articulated light arm, laser source and its optics. 


\section{B.2 Hot-film calibration set-up}

The hot-film calibration is essential for hot wire anemometry as the output of hot-film is voltage while the requirement is velocity. The calibration requires a set-up where for a known velocity measured using a standard device corresponds to a bridge voltage drop. A set of data points of known velocity and measured voltage drops is used to fit a curve using King's law. A set-up for calibrating S-type hot film is shown in Figure B.4 and is on based on the design of calibration set-up proposed by Uchiyama and Ide (2009). Few modification and adjustment is made to increases maximum velocity to $2 \mathrm{~m} / \mathrm{s}$ which more than that of Uchiyama and Ide (2009). Instead of adjustable water head for achieving variable velocity under the action of hydrostatic head, an airtight inlet chamber with wire mess to reduce turbulence is used. The hot-film calibration set-up (Figure B.4 (a)) has mainly two sections: (a) flow conditioner with nozzle and (ii) open tank for velocity measurement. Flow conditioner has a closed watertight chamber and a long pipe with nozzle fitted at one of its ends. The inlet chamber $(31.5 \mathrm{~cm} \times 23 \mathrm{~cm} \times 22.5 \mathrm{~cm})$ is made of $12 \mathrm{~mm}$ thick Perspex sheet, which is connected to the nozzle using a pipe $1000 \mathrm{~mm}$ long and $120 \mathrm{~mm}$ in diameter as shown in Figure B.4. The smaller end of the nozzle having diameter equal to $19.5 \mathrm{~mm}$ opens into the measuring tank $(500 \mathrm{~mm} \times 350 \mathrm{~mm} \times 400 \mathrm{~mm})$ at a height of $105 \mathrm{~mm}$ from bottom of tank.

To recirculate the water in calibration set-up, a water pump made of stainless steel is used. PVC pipe is used to connect the pump to the calibration tank. Suitable arrangement of diffuser and wire meshes at the selected locations is used to reduce to minimize turbulence. The jet ejects into the measuring tank having free surface at the top where the hot-film is calibrated. The nozzle issues a jet which is believed to have a potential core where the velocity remains constant for considerable axial distance. The standard velocity measuring probe (pitot tube in the present case) is kept within the potential core region so 
that the hot-film and the pitot tube sense the same axial velocity without any decay in its magnitude. The velocity at the nozzle exit can be controlled by two valves located before the inlet chamber. One valve controls the flow rate going into inlet chamber while the other one controls the bypass flow.

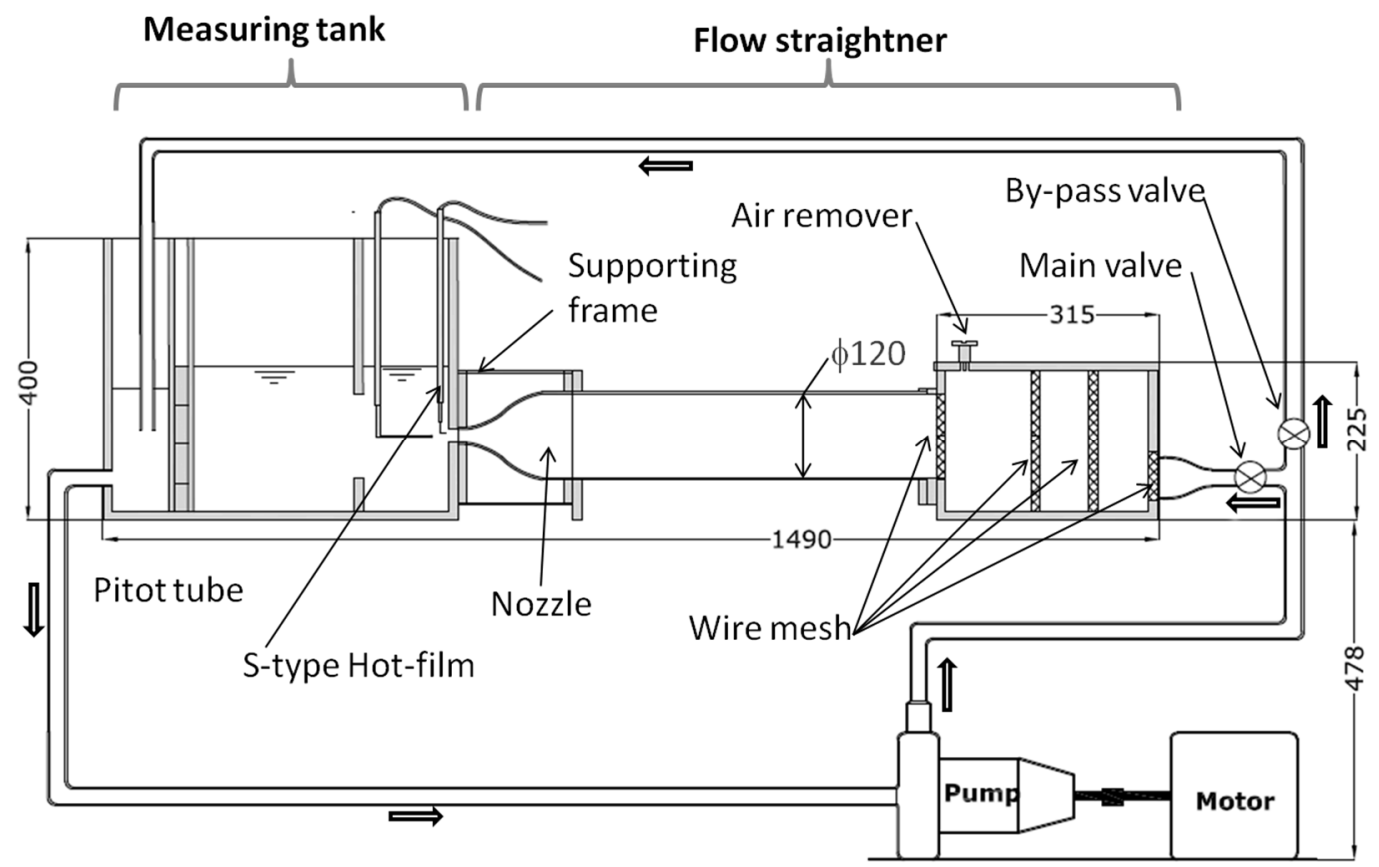

(a) Front sectional view

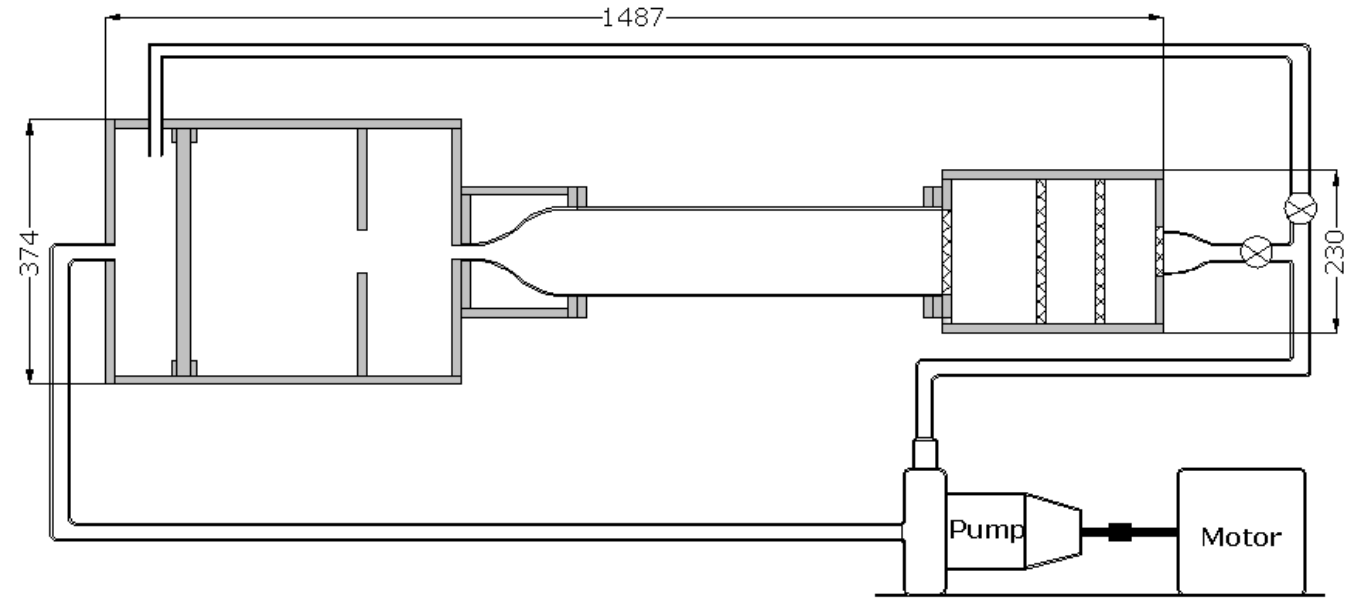

(b) Top view

Figure B.4 (a) Side and (b) top cross-sectional view of the hot-film calibration set-up 


\section{B.2.1 Nozzle design}

The accuracy of calibration procedure depends on the quality of jet coming out of the nozzle, which depends on nozzle contraction ratio, contouring of the nozzle geometry, aspect ratio and surfaces finish. At the nozzle exit, velocity profile and turbulence intensity of the jet primarily depend on the development of boundary layer inside the contraction zone of nozzle. As mentioned earlier, within the potential core region, the jet velocity remains constant (Figure B.5).

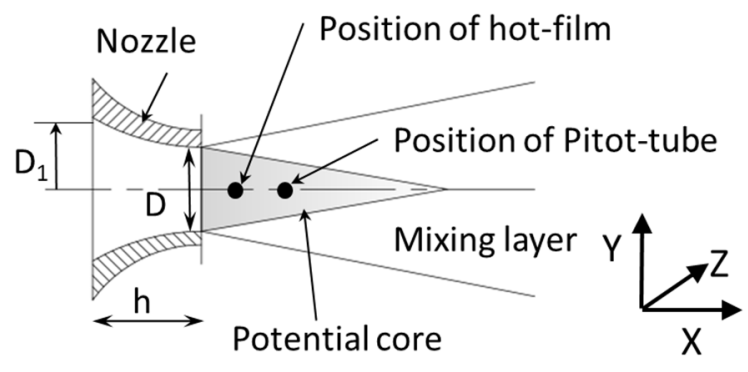

(a)



(b)

Figure B.5 (a) Potential core of jet and (b) contour profile of nozzle

For accurate calibration of the hot-film, pitot-tube must lie inside the potential core region. Assuming inviscid, incompressible and irrotational flow inside the nozzle, Davies and Jackson (1985) have shown that for a cubic arc profile nozzle a fully re-laminarized boundary layer exists nearly for all flow conditions at any value of nozzle height to its diameter (larger) ratio. The possibility of flow reversal inside the contraction region of nozzle and process of re-transition to turbulence at the nozzle exit is also less. The cubic curve equation used to generate contour shape for nozzle is

$$
R=a x^{3}+b x^{2}+c x+d
$$




$$
\begin{array}{ll}
\underline{\text { at } \mathrm{x}=0 \mathrm{~mm} ;} & \underline{\text { at } \mathrm{x}=120 \mathrm{~mm} ;} \\
\mathrm{R}=60 \mathrm{~mm}, \mathrm{dR} / \mathrm{dx}=0 ; & \mathrm{R}=10 \mathrm{~mm}, \mathrm{dR} / \mathrm{dx}=0 ; A=\pi r^{2}
\end{array}
$$

In the above expression $\mathrm{a}, \mathrm{b}, \mathrm{c}$ and $\mathrm{d}$ are constants. To solve the above equation the following boundary conditions are used. $R$ is radius of larger diameter of nozzle $\left(D_{1}\right), D$ is the smaller diameter of nozzle.

\section{B.2.2 Calibration}



(a)

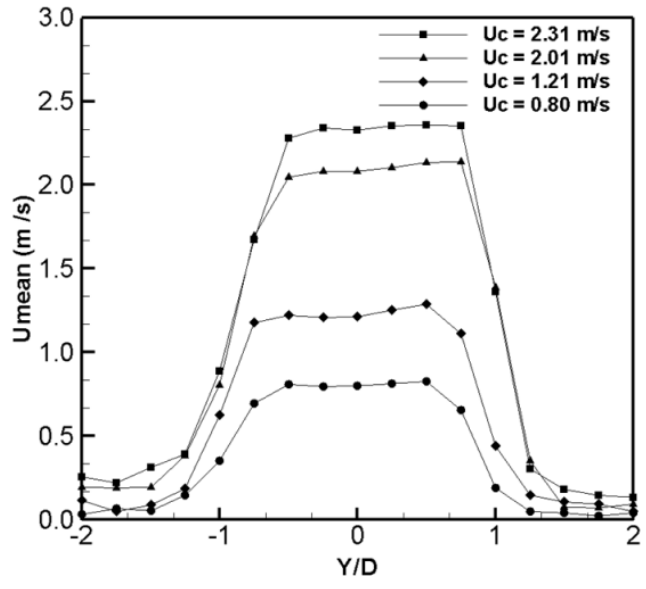

(b)



(c)

Figure B.6: (a) Hot-film calibration curve, (b) cross stream velocity profile of stream wise velocity at $0.5 \mathrm{D}$ from jet exit and (c) corresponding turbulence intensity of stream wise velocity at $0.5 \mathrm{D}$ from jet exit 
Hot film is placed at $0.5 \mathrm{D}$ away from the nozzle exit along the jet centerline in the potential core region to establish a relation between the velocities of jet and the corresponding bridge voltage of hot-film anemometer. An L-shaped pitot tube of inner diameter $1.2 \mathrm{~mm}$ is placed inside the potential core just behind the hot-film at a distance of $0.5 \mathrm{D}$. Pitot tube converts the fluid velocity into stagnation pressure, which is measured using an adjustable inclined manometer to have higher accuracy. The pressure measured as heads of water column is then converted into velocity. A voltage verses velocity plot is shown in Figure B.6 (a) for velocity range of 0 to $2.3 \mathrm{~m} / \mathrm{s}$. A fourth order polynomial fit or King's law is used for converting acquired voltage signal into velocity time trace.

\section{B.3 Water tunnel}

The water tunnel is used to test the hydrodynamic behavior of synthetic jet built-in in torpedo shape model at various crossflow velocities. The horizontal recirculating water tunnel is shown in Figure B.7, which provides an easy access to the model during experiment and can be replaced without draining the water tunnel. The overall dimension and location of various components are shown in Figure B.7 (a). The size of the test section is equal to $1.5 \mathrm{~m} \times 0.4 \mathrm{~m} \times 0.4 \mathrm{~m}$. The velocity of water inside the test section can be changed using the variable frequency drive (VFD) in the range of $0.05-0.5 \mathrm{~m} / \mathrm{s}$ (turbulence intensity $= \pm 2.5 \%$ ) at water column height of $0.35 \mathrm{~m}$. The test section provides optical access from the all four sides. 




(a)

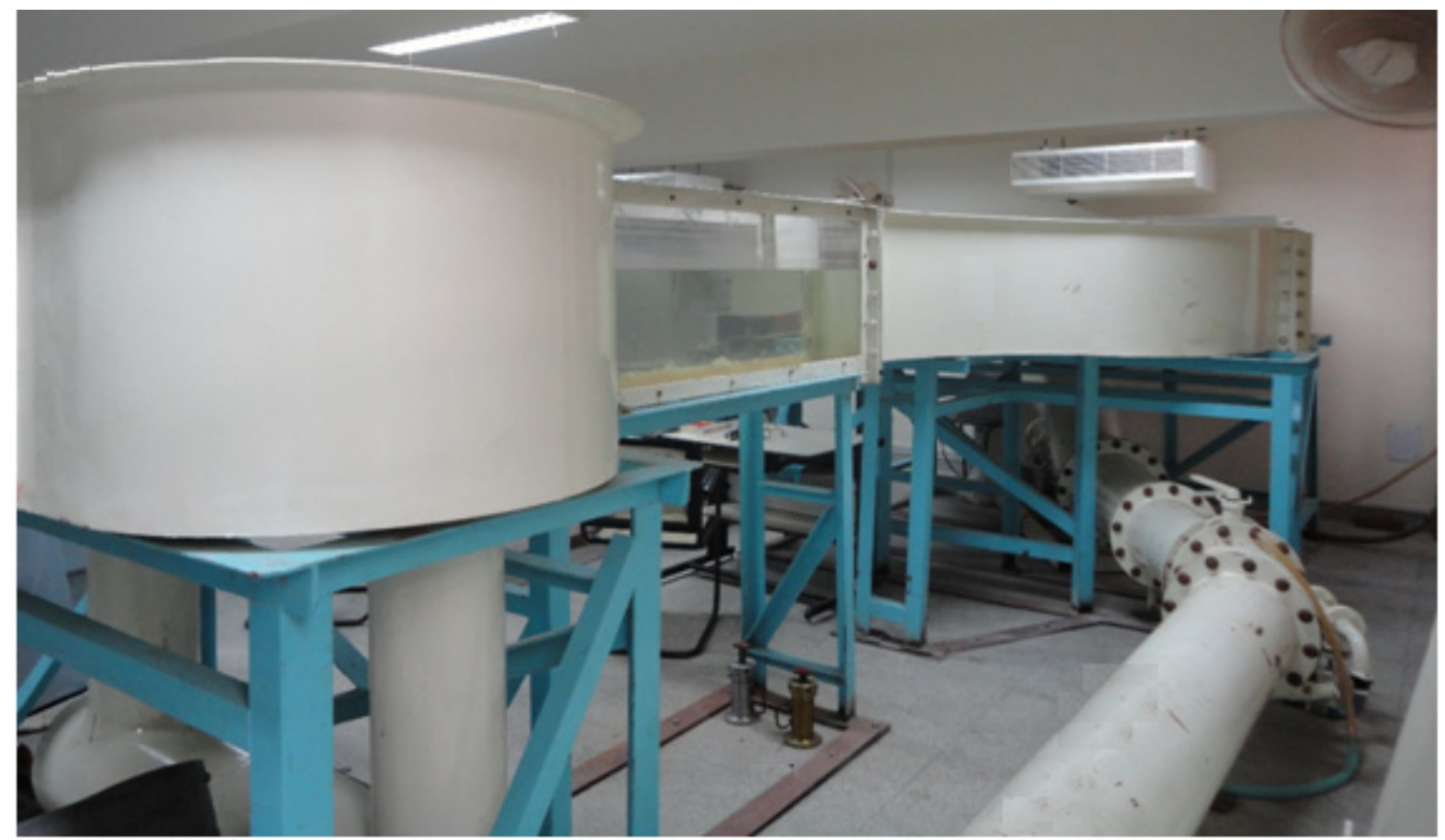

(b)

Figure B.7 (a) 3d model and (b) actual view of water tunnel 


\section{B.4 Uncertainty analysis and data reduction}

\section{B.4.1 LDV}

The basic equation for velocity measurement using LDV technique is a function of Doppler frequency $\left(\mathrm{D}_{\mathrm{f}}\right)$ and fringe width $\left(\mathrm{f}_{\mathrm{D}}\right)$. The instrument error in LDV ( $\left.U I_{\mathrm{ldv}}\right)$ due to variation in $\mathrm{D}_{\mathrm{f}}$ and $\mathrm{fD}_{\mathrm{D}}$ is $0.5 \%$ of measured velocity as given by the manufacturer. The uncertainty in the measurement of velocity is obtained from the standard deviation of 15 samples (each sample is averaged over the time of 60 seconds). The measurement error ( $\left.U I_{\mathrm{mes}}\right)$ obtained at $95 \%$ of confidence level is equal to $\pm 3.67 \%$.

$$
U I_{\text {total }}=\sqrt{U I_{l d v}^{2}+U I_{m e s}^{2}}
$$

The uncertainty (UItotal) in the velocity measurement using LDV is equal to $\pm 3.7 \%$.

\section{B.4.2 Hot film}

The effective uncertainty in velocity measured using hot-film anemometry comes from calibration curve (UIcal) and velocity reduction technique (UIred). The variation in density is less than $0.0003 \%$ due to variation in ambient temperature $\left( \pm 1^{\circ} \mathrm{C}\right)$ which is negligible and not considered. The velocity is calculated from the stagnation pressure measured using the Pitot tube opens to ambient. The minimum pressure head measured using the differential manometer is $\pm 0.01 \mathrm{~mm}$ of water. The angle misalignment of hot-film with the jet flow direction is very less and can be neglected for S-type probe. Since the hot-film and Pitot tube are placed in jet core region, stagnation pressure developed by the Pitot tube is used for velocity measurement of water jet flowing over the hot film. The effect of ambient pressure variation is neglected for calibration curve uncertainty. The calibration curve uncertainty $\left(U I_{\text {cal }}\right)$ is found to be $2.7 \%$. The uncertainty in velocity reduction $\left(U I_{r e d}\right)$ is calculated from the actual velocity $\left(\mathrm{V}_{\text {act }}\right)$ of jet measured from the Pitot tube and corresponding velocity on calibration curve using the equation given below: 


$$
U I_{\text {red }}=\sqrt{\frac{1}{n} \sum_{1}^{n}\left(\left|V_{a c t}-V_{c a l}\right| / V_{a c t}\right)^{2}}
$$

The velocity reduction uncertainty ( $\mathrm{UI}_{\mathrm{red}}$ ) obtained from the above equations is equal to

$3.1 \%$. The total uncertainty is given as:

$$
U I_{\text {total }}=\sqrt{U I_{\text {cal }}^{2}+U I_{\text {red }}^{2}}
$$

The total uncertainty is found to be $4.1 \%$ of the mean velocity.

\section{B.4.3 Actuation frequency of synthetic jet}

Quiescent flow set-up: The minimum resolution of frequency (f) displayed on frequency controller is equal to $0.1 \mathrm{~Hz}$ for the range 1 to $10 \mathrm{~Hz}$ and $1 \mathrm{~Hz}$ for the range of 10 to 100 Hz. The exact frequency imposed on diaphragm is measured using oscilloscope and compared with the frequency obtained from velocity signal. The maximum difference between these two is less than $0.05 \mathrm{~Hz}$ at $1 \mathrm{~Hz}$. The maximum uncertainty equal to $5 \%$.

Torpedo shape model: The uncertainty in the actuation frequency for frequency range 1 to $6 \mathrm{~Hz}$ is less than $5 \%$ from the mean value calculated using the measurement error between FFT of velocity and from pulses of hall-sensors.

\section{B.4.4 Dimensionless numbers}

Quiescent flow set-up: The volume of fluid expelled $\left(\mathrm{Q}_{V}\right)$ out from the orifice by forward stroke of diaphragm is measured using L-shaped glass tube of $12 \mathrm{~mm}$ inner diameter $\left(\mathrm{d}_{\mathrm{i}}\right)$ connected to the orifice (discussed in Chapter 3 in sub-section 3.1.1.3). The least count of the measuring scale on the glass tube is scale $0.5 \mathrm{~mm}$, whereas the maximum uncertainty in water column height is $1 \mathrm{~mm}$. The error in tube diameter is found to be $0.025 \mathrm{~mm}$. The uncertainty in $\mathrm{Q}_{V}$ depends on water column height $\left(\mathrm{h}_{\mathrm{w}}\right)$ and inner diameter:

$$
U I_{Q V}=\sqrt{\left(\Delta h_{w} / h_{w}\right)^{2}+2\left(\Delta d_{i} / d_{i}\right)^{2}}
$$


The uncertainty in fluid volume ejection $(\mathrm{Qv})$ is equal to $\pm 3.6 \%$. Since the slug length $\mathrm{L}$ is the function of Qv, orifice diameter (D). The uncertainty in formation number (L/D) will be

$$
U I_{L / D}=\sqrt{\left(\Delta Q_{V} / Q_{V}\right)^{2}+2(\Delta D / D)^{2}}
$$

The error in orifice diameter (D) is equal to $\pm 0.05 \%$. Substituting in above equation, the uncertainty in formation number (L/D) is found to be $\pm 6.2 \%$. Since the Strouhal number is inversely proportional to the L/D ratio, the uncertainty remains same. The Reynolds number $\operatorname{Re}=\mathrm{f}\left\{\mathrm{U}_{\mathrm{o}}, \mathrm{D}, \nu\right\}$, where the variation in density of water due to temperature change is very low and neglected. The uncertainty in $\mathrm{U}_{\mathrm{o}}=\mathrm{f}\left\{\mathrm{Qv}, \mathrm{D}, \mathrm{T}_{\mathrm{e}}\right\}$, where $\mathrm{T}_{\mathrm{e}}$ is the ejection stroke period (proportional to the actuation frequency f) having uncertainty equal to $\pm 5 \%$. Therefore the uncertainty in $\mathrm{U}_{\mathrm{o}}$ is expressed as:

$$
U I_{U o}=\sqrt{\left(\Delta Q_{V} / Q_{V}\right)^{2}+2(\Delta D / D)^{2}+(\Delta f / f)^{2}}
$$

The uncertainty obtained from the above expression in $U_{0}$ is found to be $8 \%$. Hence the uncertainty in the Reynolds number while neglecting the fluctuation in water kinematic viscosity is calculated using the equation given below.

$$
U I_{R e}=\sqrt{\left(\Delta U_{0} / U_{0}\right)^{2}+(\Delta D / D)^{2}}
$$

The uncertainty in the Reynolds number using above equation reduces to $\pm 9.4 \%$.

Torpedo shape model: The only difference in the uncertainty analysis of synthetic jet is the calculation of ejected fluid (see Equation 3.3). The diaphragm displacement is directly coupled to the cam. So the uncertainty in the diaphragm displacement is equal the uncertainty in cam ( $\pm 0.02 \mathrm{~mm}$ for $8 \mathrm{~mm}$ diameter $)$ which is $\pm 0.5 \%$. Using equation B.6 B.8, the uncertainty in $\mathrm{L} / \mathrm{D}$ ratio and Reynolds number is found to be $\pm 0.5 \%$ and $\pm 5.2 \%$. 
The uncertainty in the velocity ratio (VR) is a function of synthetic jet velocity ( $\left.U_{0}\right)$ and crossflow velocity $\left(\mathrm{U}_{\infty}\right)$. Therefore the uncertainty in velocity ratio is expressed as:

$U I_{V R}=\sqrt{2\left(\Delta U_{0} / U_{0}\right)^{2}+2\left(\Delta U_{\infty} / U_{\infty}\right)^{2}}$

The uncertainty obtained using above equation is equal to $\pm 8.2 \%$.

\section{B.4.5 Vortex core identification}

The separation distance between the counter rotating vortices are measured from the sequence of LIF images. The locus of four consecutive vortex rings is measured using imaging processing software. The orifice length (known value) appeared in the LIF images is measured in terms of pixel values and on this scale the separation distance between the counter rotating vortices are calculated. The maximum value of the range in which the locus of vortices lie divided by orifice hydraulic diameter is considered as the representation uncertainty. The equation is expressed as:

$$
U I_{\text {core }}=\text { Maximum value }\left\{\left.\left(D_{\max }-D_{\min }\right)\right|_{x}\right\} / D_{h}
$$

where $D_{\max }$ and $D_{\min }$ are the maximum and minimum distance measured between the center of counter rotating vortices at any particular downstream location ' $x$ ' for four consecutive vortex rings . Since, at 1 and $2 \mathrm{~Hz}$ actuation frequency, the vortex rings are clear and distinct in comparison to the 4 and $6 \mathrm{~Hz}$, the error in measurement of the vortex core is larger for 4 and $6 \mathrm{~Hz}$ actuation frequency. An uncertainty equal to $6.2 \%$ and $12.6 \%$ is obtained for 1 $2 \mathrm{~Hz}$ and 4-6 Hz actuation frequency respectively. 
